\title{
Image-schematic structures of near, next to and by in the landscape context of The Hobbit
}


I. Abstract

Various senses of quotidian words -not just literal or physical senses, but also figurative senses- appear very often in narrative storytelling contexts. In the case of prepositions, polysemy is an acknowledged evident phenomenon. Many linguists in the cognitive linguistics tradition have analysed the semantic contrasts between prepositions, on the grounds of image-schemas (Lakoff 1987), frames of reference (Levinson 2003) and other cognitive patterns. The aim of this paper is to clarify the meaning of the prepositions near, next to and by as well as the perception boundaries that contribute to that meaning, through the analysis of the spatial language in the book The Hobbit, by John Ronald R. Tolkien. This literary work is particularly useful for the analysis of spatial language because, by describing spatial environments and situations, the author helps readers understand an imaginary world and controls their spatial perception and conceptualisation. The term image-schema refers to imaginative procedures in connection with meaning and perception. According to Navarro i Ferrando $(2000,2006,2011)$, there are three different image-schematic perceptual dimensions in which trajector (TR) and landmark (LM) play their role for the conceptualisation of a particular scene. Thus, this research provides a proposal of prepositional meaning and polysemy regarding image-schematic structure, according to a three-dimensional system, which includes topology, force-dynamics and function to illustrate TR-LM relationships. The results shed some light on the semantic contrast between next to, near and by.

Keywords: near, next to, by, literal meaning, figurative meaning, dimensions of spatial perception, topology, dynamics, function.

\section{Introduction}

The primary purpose of this research is to analyse the three prepositions near, next to and by in contexts extracted from the book The Hobbit. Examples are analysed taking into consideration the conceptual tools of the three perceptual dimensions, i.e. topology, dynamics and function, as well as frames of reference and imageschemas, so that we better comprehend their polysemy. The relevance of this article resides in focusing on an analysis of these prepositions from the Cognitive Linguistics perspective with the purpose of unveiling their distinct meanings in the context of this story and landscape.

The relation between perception and meaning (Johnson 1987) pays attention to the interactions of the body when experiencing the 
world on the grounds of image-schemas. Thus, in association with meaning and perception, the term image-schema concerns the imaginative human mechanisms. Some authors (Lakoff 1987; Brugman 1988; Deane 1993; Sandra, and Rice 1995; Brenda 2017) use the lexical network model to show distinct and related senses of prepositions on the grounds of topological and force-dynamic relationships between TR and LM. Besides, the notion of function is used to describe the polysemy of prepositions in different languages, as Vandeloise (1991) shows for French.

In general, units embody the regularities of a language. Thus, linguists may consider regularities as constructive rules, filters or schemas. Cognitive grammar explains regularities as abstractions that take the form of schemas. Categorisation relationships connect these units so that they can form networks of any size (Langacker 2008, 219).

\subsection{Theoretical background}

\subsubsection{Aspects of image-schemas from the spatial motion group (Johnson 1987, 160-161):}

1. PAтH: the path schema consists of "parts» and "relations». This image-schema involves three components (1. a source point $A$ a starting point; 2. a terminal point $B$-a goal; and 3. a direction tracing a path between these two points- a sequence of locations connecting the source with the goal), and a relation (a force vector moving from $A$ to $B$ ). This from-to schema manifests in several types of events, such as walking from one place to another, throwing a baseball to someone, hitting someone, or even giving one's mother a gift.

2. CYCLE: this image-schema occurs along with complex interacting cycles (breathing, walking-standing, wakening-falling asleep), or also some cyclic processes (seasons, day and night, the stages of progress in animals or plants, and the life cycle itself from birth to death). The primary meaning of a cycle is that it begins at an initial point, advances through connected situations and ends at the same place where the cycle began to start a new cyclic pattern. This representation includes a climactic structure with a sine wave (rise and fall), which is named cyclic climax.

Thus, a cycle produces a spherical motion and has some features from Eviatar (1985, 161):

A cycle consists of temporal boundaries in the ordinary activities (shorten a week just to five days).

It may be sequential, multiple or overlapping (temporal for -hours or days- or functional for-cycles for doctors). 
It can be regarded in a quantitative model (time) or also a certain qualitative point (day, night, a weekend or a week). It can be natural or conventional, i.e. a traditional and cultural aspect.

Thus, both in a circular form and in the sine wave representation of a climactic structure, the cycle schema forms a basic pattern for the aim of experiencing and understanding temporal sequences and events or even non-temporal entities (numbers).

The most common image-schemas that represent force patterns are the following (Johnson 1987, 84-87, 127-135, 158):

1. COMPULSION: this image-schema means force, obligation or pressure. This situation occurs as external forces, such as wind, physical objects, water, or people move other entities. Compulsion sometimes refers to a force that overcomes resistance. Otherwise, it refers to a resisted force. This force has a direction and moves along a path.

2. BLOCKAGE: in the attempt to interact with objects or persons forcefully, this pattern occurs when an object encounters another object that blocks and resists the force. For instance, in the movement of a baby who learns how to crawl, as that baby finds a wall, it blocks the process of her journey. A force vector that encounters an obstacle represents this image-schema.

3. COUNTERFORCE: here two equally strong forces encounter each other face-to-face on the path. For instance, these forces are familiar in the football linemen, in the head-on meeting of forces.

4. Diversion: this schema shows how two forces smash or collide together with a resultant change in force vectors. For example, when someone is rowing a boat at an oblique angle against the wind, the initial force will be lost.

5. ReMOVAL OF RESTRAINT: here nothing blocks the passage. For example, when a door opens, the entrance is free to come into the room. This image-schema suggests the removal of a barrier or its mere absence.

6. ENABLEMENT: the image-schema focuses on the movements or manipulation of objects when motion brings about a sense of power or lack of power. For example, picking up an object, the groceries, but not lifting the front of a car. Here the usual gestures for force are included in this structure of possibility, including a potential path of motion. Accordingly, this imageschema is represented by a potential force vector or absence of an obstacle.

7. ATtRACTION: an object moves on the path under an attracting force. For example, in the case of a magnet that draws metal toward itself, when a vacuum pulls dirt into itself, or the earth 
pulls someone back down, as she jumps. This force is a kind of gravitation towards an object, and sometimes there may be other additional objects incorporated in the relation of attraction.

8. LINK: this image-schema occurs when of the linked objects are in contact. These objects connect by concrete or abstract linkages with the combination of perceptual capacities or the situation of the perceptual environment. Thus, a link appears in common physical cases, such as when the lump fits into the wall socket as an electrical fitting, the snaps of the coat join, or a child holds the parent's hand. In the structure of the link schema, there are two elements, A and B, linked by a bounding area.

Thus, linkages may not only be physical and spatial bindings or attachments, but also temporal connections (a temporal sequence), causal connections (a network is seen as a set of links to comprehend a place), genetic connections (a connection via the link of a common source), or functional linking (an object may have a relation with a functional unity).

In the balance group, there are image-schemas of axis balance, point balance, twin-pan balance or equilibrium. A prototypical balance schema consists of force vectors, which represent weight, an axis or point about the distribution of forces. This balance includes a symmetrical or proportional organisation of forces close to a point and axis. In all expanded senses of balance based on this original schema, both the axis and force vectors maintain a metaphorical extension.

1. AXIS BALANCE: force vectors and an axis represent this imageschema.

2. POINT BALANCE: the symmetrical force vectors decrease to two symmetrical vectors since the equivalent weights from the balancing force vectors are adhering at a point. Equivalent weights occur when the forces are the same on each side of the supporting point.

3. TWIN-PAN BALANCE: the axis decreases to an essential point that functions as a support. In the balance of a rational argument, for instance, the winner of an argument feels the balance tip in her favour. In the legal balance, the judge must estimate the weights of damages and order a fine in some way identical to the damages as compensation, or at least «let the punishment fit the crime». In mathematical equality, whatever is included to one side, the same must be included to the other side to maintain equality.

4. EqUILIBRIUM: the symmetrical force vectors encounter at a point onto a surface of curvature, or a sphere (container-surface). Here there is a balance of the internal and external forces, which 
relates to a three-dimensional container. In the systematic balance, for example, the circulatory or respiratory system of our body must be in a dynamic equilibrium and a balance of forces with the purpose of functioning correctly. In the psychological balance, as an emotional experience, for instance, people usually seek an emotional balance, and the emotions must be under control.

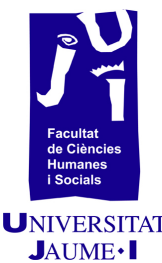

\subsubsection{Transformational aspects of image-schemas (Lakoff 1987, 416-460):}

Transformational aspects of image-schemas may contribute to focus attention on specific of a scene for the elaboration of a concept:

a. PATH-FOCUS to END-POINT-FOCUS: in the imaginary, this operation implies to come after the moving object in a path, and then, focus on the point where this object comes to a stop, or where it will proceed to rest.

b. MULTIPLEX TO MASS: if we imagine and we look at a group of various objects or individuals, the fact of moving away from the group makes it become a single and alike mass when the identity of individuals fades away, and therefore the perception provides just a mass. Then, we may move back to the position where the mass (cattle) turns again into a collective or multiplex entity (cows).

c. Following a tRAJECTORY: a moving object is perceived continuously; then, in mind, we can indicate the path it follows or the trajectory it is about to follow.

d. SUPERIMPOSITION: there is a large sphere and a small cube in a scene, the size of the cube increases until the sphere fits inside it. Then, the size of the cube decreases and fits within the sphere.

e. Reflexive: This operation can be easily described using the preposition out. For instance, Harry ran out of the room. In this situation, the TR moves from the interior of the room to the exterior. The syrup spread out. When someone pours the syrup on the table, its boundary moves, since part of that motion was inside the boundary, and after that motion, part of the syrup is outside the previous boundary. That connection between the TR and the $L M$ is called reflexive. The term reflexive $T R$ refers to an entity. In some cases, also, a part of a unique entity appears as a TR, and another part of that entity appears as an LM. For instance, roll the log over. Here the path indicates the object moving above and across, called reflexive path. The reflexive TR appears in this sentence of over since half of the log appears as TR and the other half as an LM as it rolls over.

f. ROtATION: In this transformation, the TR blocks access of vision to at least a part of the LM. There is a continuous motion and, in 
some cases, a lack of orientation. For instance, there were flies all over the ceiling. As the rain came down, it froze, and ice spread all over the windshield.

In the spatial group, Lakoff describes further aspects of schemas (Lakoff 1987, 104; 276; 419-428):

a. COVERING: there are quantifiers mixed with over, such as all to describe the covering schema. These quantifiers occur with masses (most wine) and multiplex entities (mostly ducks). At that point, imagine individual cows in a field where the perception occurs as multiplex entities. After going away from this field, the perception is a mass. For instance, I walked all over the hill. Here the path covers the LM through a collection of points when the TR is moving along that path.

b. AвOVE: this schema has no specific path and no boundaries. Here the contact sense does not occur between a TR and an LM, as in some examples such as the helicopter is hovering over the hill. Here the helicopter is moving above the LM. When the helicopter lands, it is not over the hill, at that moment it is on the hill.

c. Across: This aspect specifies the above/across sense. The preposition over matches both above and across. An example of it is the plane flew over. Here the plane is the TR. At this point, the LM is omitted. However, the path is above the LM, also across the boundary from one point to the opposite, and there is no contact between the TR and the LM.

d. CONTACT: this schema refers to a situation where a TR and an LM touch. For instance, Sam walked over the hill. A hill is extended and vertical, and the action of walking requires contact with the ground.

e. Vertical orientation: a metaphor consists of a source domain, a target domain, and a source-to-target mapping. When a metaphor is natural, the structure of our experience guides its development. The phrases more is up and less is down describe this vertical orientation, for instance, the number of books published each year keeps going up. That stock has fallen again. The source domain is verticality, and the target domain is quantity. Here the domain of verticality correlates with the updown schema, which functions in association with gravity.

f. LENGTH (extended TR): For instance, the bird flew over the yard. The telephone line stretched over the yard. In the latter example, over describes the extension of a long, thin TR such as the telephone line. Let us look at another example like the rocket shot up, in contrast with the lamp was standing up. Here, in the first case, up is used to indicate the motion path of a TR, and in the second case up is used for the extension of a long, thin 
object such as the lamp. These structures suggest that an image-

schema transformation exists, considering a motion schema where a TR moves, and a long, thin object schema as extended. These schemas establish a basis for category extension.

\subsubsection{Frames of reference and dimensions}

According to Levinson (2004), there are three primary frames of reference, namely intrinsic, relative and absolute. Table 1 briefly summarises their definitions:

Table 1. Frames of reference (based on Levinson 2004, 76)

\begin{tabular}{|c|c|c|}
\hline I & HORIZONTAL DIRECTION & \\
\hline INTRINSIC & RELATIVE & ABSOLUTE \\
\hline $\begin{array}{l}\text { A canonical position. } \\
\mathrm{t}\end{array}$ & A perception from a viewpoint. & The gravity axis. \\
\hline $\begin{array}{l}\text { Debcription: a domain } \\
\text { from a named side of a } \\
\text { landmark object. }\end{array}$ & $\begin{array}{l}\text { Description: the observer's axes } \\
\text { mapped onto the ground object } \\
\text { which are named angles. }\end{array}$ & $\begin{array}{l}\text { Description: a fixed set } \\
\text { of sides and axes for } \\
\text { defining a direction from } \\
\text { a ground object. }\end{array}$ \\
\hline
\end{tabular}

Horizontal perspective (Levinson 2004), the conceptual puzzle is to define angles which can project onto a ground object. Levinson answers to this puzzle describing three frames of reference. Firstly, the intrinsic system is projected out by a domain from a named side of a landmark object. Secondly, the observer's axes describe the relative system, and these axes are mapped onto the ground object and are named angles. Finally, the absolute system uses a fixed set of sides and axes to define a direction from a ground object.

In addition, from a Cognitive Linguistics perspective, Navarro i Ferrando $(1998,2006,2011)$ provides a model for the semantic representation of prepositions whose senses are derived and arranged according to three semantic dimensions:

1. Topology: The visual perception of objects gives the speaker clues for establishing and conceptualising topological relations like coincidence, contact, inclusion, proximity, and the like.

2. FORCE-DYNAMICS: Human beings have experience of self-motion and object motion, which provides the clues for conceptualising patterns of interaction concerning dynamics.

3. FUNCTION: Human beings have experience of the effects of interaction, as well as the consequences of those effects for survival and well-being. (Navarro 2006, 171).

\section{Objectives}

Regarding semantics, the primary objective of this research is to perform a detailed analysis of the prepositions near, next to and by, 
with the purpose of unveiling the semantic distinctions that make them different.

Firstly, a view of the theoretical background about perceptual dimensions, frames of reference and image-schemas is necessary, as introduced in the previous sections. Secondly, a contextual analysis of these three prepositions from contexts in The Hobbit is carried out. Thirdly, comments on some examples illustrate the semantic dimensions studied (Johnson 1987; Lakoff 1987; Levinson 2003; Navarro i Ferrando 2000, 2006, 2011).

\section{Material and method}

The material used is the novel The Hobbit, from which we select contexts including the three prepositions near, next to and by. Their definitions from dictionaries (Oxford Dictionary and Cambridge Dictionary) provide the first account of their diverse senses. Subsequently, the analytical tools taken into account are imageschemas for topology, dynamics and function. Next, some examples from the book The Hobbit are analysed according to these three dimensions, including figurative senses derived from them. Finally, we perform an analysis of each example including the corresponding dimension, the frame of reference and image-schema as well as the predominant TR and LM types.

The procedure is as follows:

- The first step consists in extracting the first 50 fragments including prepositions near, next to and by from The Hobbit.

- The second step consists in the analysis of semantic dimensions, topology, dynamics and function, frames of reference and image-schemas.

- Finally, the semantic contrasts between near, by and next to are identified.

\section{Results}

Each preposition (near, next to and by) is described along the three dimensions of spatial perception, i.e. topology, dynamics and function, in the landscape contexts extracted from the novel. A detailed meaning description provides for a contrast between these prepositions.

Firstly, the dictionary definitions of the three prepositions are presented and elaborated along the three perceptual dimensions. Secondly, an analysis of examples extracted from The Hobbit illustrates the meanings and senses grounded on the perceptual dimensions under elaboration.

Senses of preposition near in English Oxford Dictionary: 
1. At or to a short distance away from (a place): «do you live near here?», [superlative] "the table nearest the door».

2. A short period of time from: "near the end of the war». [comparative] "details will be given nearer the date».

3. Close to (a state): «she gave a tiny smile, brave but near tears».

3.1 A small amount below (another amount): «temperatures near 2 million degrees.»

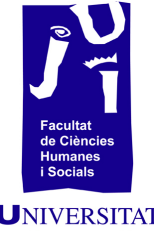

4. Similar to: "a shape near to the original».

Senses of the preposition near in the Cambridge Dictionary:

1. Not far away in the distance: "Is there a restaurant near here?». "I'd like to sit near a window, please".

2. Not far away in time: «We can decide which route to take nearer the time».

3. Almost in a particular state, or condition: «the runners looked near exhaustion».

According to the senses found in the dictionaries, the meaning of near expresses the following perceptual dimensions:

TOPOLOGY: According to the topological configuration of the TRLM scene, a TR is located in a position to a short distance in respect to an LM. A metaphorical sense can occur for time expressions and emotional states as locations. Also, the sense of nearness in a scale of quantity and quality is expressed by this preposition.

DYNAMICS: In this dimension, both horizontal and vertical axis can occur in these landscape contexts using this preposition. Therefore, this preposition leaves the motion axis unspecified because of the direction of movement. Thus, the preposition near allows for any direction of motion according to the examples analysed.

FUNCTION: In this dimension, an LM is accessible to a TR. But this TR does not imply intentionality or control of the landscape context in a scene.

The examples of near are as follows:

1. «There was a little cave (a wholesome one with a pebbly floor) at the foot of the steps and near the end of the stony ford. Here the party gathered and discussed what was to be done» (p. 191, line 20).

Here the topological dimension is clearly expressed, which means proximity to an entity in a short distance. The frame of reference is absolute, and the image-schema is centre-periphery. The TR (a little cave) is more salient than the LM (the end of the stony ford).

2. «He was a descendant in long line of Girion, Lord of Dale, whose wife and child had escaped down the Running River from the ruin long ago. Now he shot with a great yew bow, till all his arrows but one were spent. The flames were near him. His 
companions were leaving him. He bent his bow for the last time» (p. 399, line 12).

Here the dynamics dimension is more prominent, which means there is an apparent movement. The reference frame is relative. The axis is undetermined. The image schema indicates a trajectory. The TR (flames) is more salient than LM (him).

3. «Every now and again, while he was still near enough, they caught a glint and a tinkle as he stumbled on some golden thing. The light grew smaller as he wandered away into the vast hall; then it began to rise dancing into the air. Bilbo was climbing the great mound of treasure. Soon he stood upon the top, and still went on» (p. 378, line 12).

The most prominent dimension here is function, which expresses accessibility (start across, wandered, treasure). The frame is relative. The image-schema shows source-path-goal (from-to). The TR (he) is less salient than LM (mound of treasure).

4. "'We will have King Bard!" the people near at hand shouted in reply. "We have had enough of the old men and the moneycounters!" And people further off took up the cry: "Up the Bowman, and down with Moneybags," till the clamour echoed along the shore» (p. 402, line 25).

The dimension is function, which means interaction and accessibility (shouted). There is a figurative sense: near at hand (close, approaching). The frame is relative. The image-schema is expresses centre-periphery. The TR (the people) is salient, and the LM is not specified in the sentence but further below in the context (along the shore).

The preposition next to in the Cambridge Dictionary:

1. Used when describing two people or things that are very close to each other with nothing between them. "Can I sit next to the window?»

2. Used to mean "after» when making a choice or a comparison: "cheese is my favourite food and, next to that, chocolate».

3. Almost: «they pay me next to nothing (very little), but I really enjoy the work».

The preposition next to in the English Oxford Dictionary:

1. Following in order or importance: «next to buying a new wardrobe, nothing lifts the spirits like a new hairdo!»

2. Almost: "I knew next to nothing about farming."

3. In comparison with: «next to her, I felt like a fraud.»

According to the senses found in the dictionaries and the contexts analysed in our corpus, the meaning of next to expresses the following perceptual dimensions: 
TOPOLOGY: according to this dimension a TR is in a contiguity position about an LM. Contact is acceptable, but it does not occur on many occasions. There may be also other entities in the context besides a TR and an LM. The closest entity to an LM is a TR in the scene.

DYNAMICS: In this dimension, the horizontal axis is relevant because of the direction of movement. At this point, the preposition next to implies this direction according to the examples regarded.

FUNCTION: A TR implies intentionality and attention to an LM in a scene. The fact that an entity is very close to another entity makes it possible for a human perceiver to compare both entities with each other. This fact allows for the metaphorical extension of the function "comparison", so that in non-physical domains next to indicates a relationship between two entities as compared from the speaker's point of view. A metaphorical sense can occur for comparison, or for comparing the importance of TR and LM.

The examples of next to are:

1. «Half a minute! said Dori, who was at the back next to Bilbo, and a decent fellow. He made the hobbit scramble on his shoulders as best he could with his tied hands, and then off they all went at a run, with a clink-clink of chains, and many a stumble, since they had no hands to steady themselves with» (p. 116, line 2).

The topological dimension expresses contiguity. The reference frame is intrinsic, because of the inferred lateral side of the LM. The image-schema is seen as centre-periphery. The TR (Dori) is equally salient as the LM (Bilbo).

2. "I see they have begun to arrive already," he said when he caught sight of Dwalin's green hood hanging up. He hung his red one next to it, and "Balin at your service!" he said with his hand on his breast» (p. 30, line 7).

The dynamics dimension expresses motion. The frame is intrinsic. The schema is force (Johnson, 1987). The TR (his red hood) is less salient than the LM (green hood).

3. «As he lay in bed he could hear Thorin still humming to himself in the best bedroom next to him» (p. 59, line 10):

The function dimension implies interaction and perception (to sing with closed lips). The frame is relative. The schema is centre-periphery. The TR (Thorin) and the LM (him) are in a symmetrical relation.

4. «It was now nearly lunchtime, and most of the things had already been sold, for various prices from next to nothing to old songs (as is not unusual at auctions). Bilbo's cousins the Sackville- Bagginses were, in fact, busy measuring his rooms to see if their own furniture would fit» (p. 483, line 23). 
The topology dimension indicates proximity. The author makes a comparison. The frame is relative. The image-schema a scale. The TR (various prices) is less predominant than the LM (at auctions).

Next to nothing is a metaphor which means almost nothing, a very small amount (idiom).

The preposition by in the Cambridge dictionary:

1. Agent: used to show the person or thing that does something. "The motorcycle was driven by a tiny bald man".

2. Method: used to show how something is done. «They travelled across Europe by train/car».

3. Position: near or at the side of something. "A small child stood sullenly by her side».

4. No later than: (not later than; at or before) «she had promised to be back by five o'clock».

5. Measurement:

- Used to show measurements or amounts: "our office floor space measured twelve meters by ten" (= was twelve meters in one direction and ten in the other).

- Used to say that something increases or changes with every minute, hour, day, etc. that passes: "the death toll from the hurricane was climbing minute by minute/by the minute».

6. During: «We travelled by night and rested by day».

- Used when describing someone's character or job: «she is, by nature, a sunny, positive kind of person".

- If something is all right/fine by someone, they agree that it can happen: «l'd rather go later. That's fine by me».

The preposition by in the English Oxford Dictionary:

1. Indicating the amount or size of a margin: "the shot missed her by miles».

2. Indicating the location of a physical object beside a place or object: "remains were discovered by the roadside».

3. Indicating the amount or size of a margin: "the shot missed her by miles».

By expresses the following perceptual dimensions:

TOPOLOGY: In this dimension, a TR is in a proximity/contiguity position from an LM. A metaphorical sense can occur for time expressions (no later than, by night) and also for some increases or changes in measurement. There are also other entities in a scene, but these are irrelevant.

DYNAMICS: In this dimension, the horizontal axis is relevant because of the direction of movement. At this point, the preposition by implies this direction according to the examples regarded. 
FUNCTION: In this dimension, an instrument or an agent used with the passive voice occurs on many occasions. Then, a TR implies intentionality and attention to an LM in the landscape context from a scene.

The examples of the preposition by are:

1. "'Bother burgling and everything to do with it! I wish I was at home in my nice hole by the fire, with the kettle just beginning to sing!» It was not the last time that he wished that!» (p. 66, line 25).

The topology dimension expresses proximity. The frame is relative. The TR (I) is less salient than the LM (the fire).

2. «In fact, he was just sitting down to a nice little second breakfast in the dining-room by the open window, when in walked Gandalf» (p. 63, line 2).

The dimension is dynamics, which means stative. The frame is relative. The image-schema is a balance. Here there is no motion. The axis is considered to be horizontal. The TR (he) is less predominant than the LM (the open window).

3. «The chance never arrived, until Bilbo Baggins was grown up, being about fifty years old or so, and living in the beautiful hobbit-hole built by his father, which I have just described for you, until he had in fact apparently settled down immovably» (p. 23, line 16).

The functional dimension expresses control. The reference frame is relative. The image-schema is centre-periphery. The TR (his father) is less predominant than LM (the beautiful hobbithole).

\section{Discussion and conclusions}

The most frequent image-schemas underlying the use of the preposition near are centre-periphery and source-path-goal (fromto). In addition, the frame of reference is mostly relative.

As for the preposition by, the image-schemas are centreperiphery and balance, and the frame of reference is mostly relative.

As for the preposition next to, the image-schemas are scale, centre-periphery and balance. The frame of reference may be relative or intrinsic.

Regarding the dimension of topology:

$\infty$ The preposition near means proximity. The TR is more salient than the LM. Some entities may be frequently conceptualised as located between the TR and the LM. However, the preposition does not specify whether there are other entities between the TR and the LM or not. 
$\infty$ The preposition next to means contiguity, including contact in some contexts. The TR and the LM are conceived symmetrically. The preposition does specify that there are no other entities between the TR and the LM.

$\infty$ The preposition by means proximity, and contiguity in some contexts. The TR is less salient than the LM. The preposition does specify that there are no other entities between the TR and the LM.

Referring to the dimension of dynamics:

$\infty$ The preposition near means no parallel orientation. There may be motion.

$\infty$ The preposition next to means mutually lateral orientation. There is a balance and, usually no motion.

$\infty$ The preposition by means no mutual orientation. There is a balance and no motion.

As to the dimension of function:

$\infty$ The preposition near means no mutual attention, but the preposition does indicate accessibility. There is an intentional TR.

$\infty$ The preposition next to means no mutual attention, and there is a judgement of comparison on the part of the conceptualiser.

$\infty$ The preposition by means attention, control and intentionality.

This study is but a first attempt or pilot research on the perceptual dimensions that define the use of the three prepositions, near, next to and by. Further research with larger corpora are necessary to corroborate the hypotheses stated here.

\section{References}

Brenda, Maria. 2017. "A Cognitive Perspective on the Semantics of Near.» Review of cognitive linguistics 15 (1): 121-153.

Brugman, Claudia. 1988. "The story of Over: Polysemy, Semantics, and the Structure of the Lexicon.» M.A. Thesis, University of California.

Cambridge Dictionary. n.d. "Cambridge Dictionary.» Accessed Month Day, Year. https://dictionary.cambridge.org/es/.

Deane, Paul D. 1993. «At, by, to and past: An Essay in Multimodal Image Theory". Proceedings of the Annual Meeting of the Berkeley Linguistics Society 19: 112-124.

Eviatar, Zerubavel. 1985. The Seven Day Circle: The history and meaning of the week. New York: Free Press. 
Johnson, Mark. 1987. The body in the mind: The bodily basis of meaning, imagination, and reason. Chicago: The University of Chicago Press.

Lakoff, George. 1987. Women, Fire and Dangerous Things. What Categories Reveal about the Mind. Chicago: The University of Chicago Press.

Langacker, Ronald W. 2008. Cognitive Grammar. A Basic Introduction. Oxford: Oxford University Press.

Levinson, Stephen C. 2004. Space in Language and Cognition. Explorations in Cognitive Diversity (Language, Culture and Cognition). Cambridge: Cambridge University Press.

Oxford English Dictionary. n.d. "Oxford English Dictionary.» Accessed Month Day, Year. https://en.oxforddictionaries.com/.

Navarro i Ferrando, Ignasi. 2000. "A Cognitive-Semantic Analysis of the English Lexical Unit In.» Cuadernos de investigación filológica XXVI: 189-220. Universidad de La Rioja.

Navarro i Ferrando, Ignasi. 2006. «On the meaning of three English prepositions." In In-roads of Language: Essays in English Studies, edited by Ignasi Navarro, and Nieves Alberola, 167-179. Castelló: Universitat Jaume I.

Navarro i Ferrando, Ignasi, and Gösser, Birgit. 2011. "Semantic configuration of the spatial concept "Behind" in English.» Studia Universitatis Petru Maior Serie Philologia 10: 204-209. Târgu Mures,: Editura Universitatii Petru Maior.

Sandra, Dominiek, and Rice, Sally. 1995. "Network Analyses of Prepositional Meaning: Mirroring whose mind - the linguist's or the language user's?» Cognitive Linguistics 6 (1): 89-130.

Tolkien, Ronald R. J. 2009. The Hobbit. HarperCollins Publishers.

Vandeloise, Claude. 1991. Spatial Prepositions: A Case Study from French. Chicago: University of Chicago Press. 
The data presented in the study consisted of 44 business emails that contained a variety of requests made to coordinate the stuffing of the containers on the vessel, arrange all the documentation, and follow shipment until the vessel arrived at the port of discharge. Two types of requests were identified - requests for action and requests for information.

The present study aimed to investigate the speech act of requesting reproduced in business correspondence in the field of Maritime English, following the analytical framework of Trosborg (1995). Her taxonomy for requests, previously illustrated, consists of four different categories where the requests can be classified depending on their directness or indirectness.

\section{Results}

Two different sets of conversations were analysed taking into account the different participants and the table previous explained by Trosborg (1995). Results are going to be presented in two different tables (Table 4 and Table 5) according to each participant; and below the examples of each category indicating the type of request underlined in the correspondence and the set of conversation it belongs. Finally, Table 6 indicates the types of modification devices found in the requests analysed.

Table 4. Results of Participant 1

PARTICIPANT 1

\begin{tabular}{|l|c|}
\hline \multicolumn{1}{|c|}{ CATEGORIES } & TOTAL \\
\hline A. DIRECT & 4 \\
\hline Performatives & 33 \\
\hline Imperatives & \\
\hline B. CONVENTIONALLY INDIRECT (HEARER-BASED) & 7 \\
\hline Ability & 2 \\
\hline Suggestory formulae & \\
\hline C. CONVENTIONALLY INDIRECT (SPEAKER-BASED) & 1 \\
\hline Desires / Needs & \\
\hline D. INDIRECT & 4 \\
\hline Hints & \\
\hline
\end{tabular}

Email examples:

Example 1. A. Direct - Performatives - Set 3, Email 1

EMAIL 1 
Dear Laura,

Hope you are doing well.

Just got the new inquiry that I would like you to quote us the offshore and onshore services for the shipment details

POD: Hochiminh port (HCM City) or Cai Mep port (Vung Tau), please offer for 2 options for client consideration.

** Please also adv the name of loading port, transit time, frequency, shipping line name \& validity.

Your prompt action would be highly appreciated.

Should you have question, please inform us.

Name of Participant 1

Example 2. A. Direct - Imperatives - Set 2, Email 5

\section{EMAIL 5}

Dear Laura,

Please re-check the schedule of for LINE Z, why the gap from Closing time (11/06) and ETD (21/06) is so far? Please check if ready date 01 Jun, can you catch the schedule ETD 07 Jun of LINE $Z$ ?

As per your below message, LINE $P$ carrier have soonest schedule, right? Please adv.

Name of Participant 1

Example 3. B. Conventionally indirect (Hearer-Based) - Ability - Set 1, Email 6

\section{EMAIL 6}

Dear Laura,

Any news for this inquiry? Can we have the rates within today?

Name of Participant 1

Example 4. B. Conventionally indirect (Hearer-Based) - Suggestory formulae - Set 3, Email 9

\section{EMAIL 9}

Dear Laura,

Well noted, thanks for your rates on stuffing/vanning/securing.

How's about the charges for LTHC + B/L + ISPS + Seal + Wharfage + VGM+Export customs? Last time, you offered in lumpsum charge, now you should break down and adv us the separate charge, right?

Name of Participant 1

Example 5. C. Conventionally indirect (Speaker-Based) - Desires/Needs - Set 2, Email 1 


\section{EMAIL 1}

Dear Laura,

Any news? we need to reply client now, kindly revert URGENT. Thanks. Name of Participant 1

Example 6. D. Indirect - Hints - Set 1, Email 1

\section{EMAIL 1}

Dear Laura,

Referring to below message from my colleague, We have an inquiry for exwork shipment from Spain to HCM port

Please adv all relevant charges, Transit time, frequency and validity.

Please consider the suitable port of loading and offer us the best freight soon..

Should you need any information, please freely let me know.

Appreciated for your any feedback

Thanks

Name of Participant 1

Table 5. Results of Participant 2

PARTICIPANT 2

\begin{tabular}{|l|c|}
\hline \multicolumn{1}{|c|}{ CATEGORIES } & TOTAL \\
\hline A. DIRECT & 1 \\
\hline Performatives & 21 \\
\hline Imperatives & \\
\hline B. CONVENTIONALLY INDIRECT (HEARER-BASED) & 3 \\
\hline Ability & 1 \\
\hline Permission & \\
\hline C. CONVENTIONALLY INDIRECT (SPEAKER-BASED) & 1 \\
\hline Desires / Needs & \\
\hline D. INDIRECT & \\
\hline Hints & \\
\hline
\end{tabular}

Email examples:

Example 1. A. Direct - Performatives - Set 2, Email 21

\section{EMAIL 21}

Dear Laura,

Regarding to 1 st \& 2nd shipment, client is worry that cargo will be damaged in transit, so they asked us to provide some more pictures 
of VANNING/SECURING cargo inside containers for checking, can you provide us soon?

Thanks \& Awaiting for your news.

Name of Participant 2

Example 2. A. Direct - Imperatives - Set 2, Email 15

\section{EMAIL 15}

Dear Laura,

Consignee on $\mathrm{Mbl}$ is ok now. However, pls make sure all information on description of goods/ packages/ cbm between $\mathrm{M} / \mathrm{bl} \& \mathrm{H} / \mathrm{bl}$ must be matched together. Pls issue draft $\mathrm{H} / \mathrm{bl}$ for our checking again to avoid any incurred charge, thanks a lot

Name of Participant 2

Example 3. B. Conventionally indirect (Hearer-Based) - Ability - Set 2, Email 22

\section{EMAIL 22}

Dear Laura,

We mean if you did the dunnage \& lashing cargo when stuffing, can you send pictures to customers to see and tranquilize?

Name of Participant 2

Example 4. B. Conventionally indirect (Hearer-Based) - Permission Set 2, Email 26

\section{EMAIL 26}

Dear Laura,

May we know if you have got any update from shipping line for this vessel, it has been departed as plan ETD BCN 31 May or any delay? PIs check \& advise us within today because we need to update to our customer for status cargo for their following

Name of Participant 2

Example 5. C. Conventionally indirect (Speaker-Based) - Desires/Needs - Set 2, Email 26

\section{EMAIL 26}

Dear Laura,

May we know if you have got any update from shipping line for this vessel, it has been departed as plan ETD BCN 31 May or any delay? PIs check \& advise us within today because we need to update to our customer for status cargo for their following

Name of Participant 2

Example 6. D. Indirect - Hints - Set 2, Email 17 
EMAIL 17

Dear Laura,

1. Pls kindly see our comment as attached file per our remark by red words. For this shipment, pls correct type of freight is FREIGHT PREPAID on $\mathrm{H} / \mathrm{bl}$ help us ( We will collect $\mathrm{O} / \mathrm{F}$ and all destination surcharges from third party ( notify party ) at our side, so you don't need to collect O/F from shipper on behalf of us, pls note )

2. We have corrected FREIGHT PREPAID by hand first on $\mathrm{H} / \mathrm{bl}+\mathrm{M} / \mathrm{bl}$ to sign \& stamp as your request. Pls refer as attachment

Name of Participant 2

Table 6. Results of the use of softeners in direct imperative forms

\begin{tabular}{|l|c|c|}
\hline \multicolumn{1}{|c|}{ CATEGORIES } & \multicolumn{2}{c|}{ TOTAL } \\
\hline & PARTICIPANT 1 & PARTICIPANT 2 \\
\hline A. DIRECT - IMPERATIVE SOFTENERS & 4 & 2 \\
\hline HELP & 5 & \\
\hline KINDLY & 2 & \\
\hline FREELY & 2 & \\
\hline
\end{tabular}

Email examples:

Example 1. A. Direct imperative softeners - Help - Participant 2 - Set 2, Email 19

\section{EMAIL 19}

Dear Laura,

Your invoice is ok now. As per schedule, ETD BCN is today - 31 May 2018 , pls help to check if the vessel has been lelf out of Barcelona already or not? Pls update the status for us soon

Name of Participant 2

Example 2. A. Direct imperative softeners - Kindly - Participant 1 - Set 3, Email 2

\section{EMAIL 2}

Dear Laura,

As per incoterm 2010, Exwork charges is inclusive the job of loading cargo onto truck at shipper premise, please help to re-check and adv if below charges have included loading charge or not? If not, kindly also offer to us soon.

Name of Participant 1 
Example 3. A. Direct imperative softeners - Freely - Participant 1 - Set

1, Email 5

\section{EMAIL 5}

Dear Laura,

* Client asked to give the free time at destination is 14 days of demurrage, please check and try to negotiate with shipping line if they can match with client requirement?

Should you need any information, please freely let me know.

Appreciated for your any feedback within today!

Name of Participant 1

\section{Discussion and conclusion}

The entire world is connected through international trade. Consequently, the shipping industry is very important in the export and import flow of cargo all over the continents. Hence, the knowledge of the processes of overseas shipments by forwarders is crucial to link, without any breakdown, all the blades of the procedure.

The aim of this article was to analyse the formality and informality expressed in Vietnamese correspondence, according to the information exchanged to arrange three maritime exportation traffics, with the purpose of identifying if the directness or indirectness when writing depends on the knowledge of both the exportation process and the language.

With regard to the type of direct request, each participant uses them, being the request most performed along the email correspondence. In some cases, Participant 1 and Participant 2 adopt direct performative requests; however, it is the direct imperative request the one most employed. In addition to the above-mentioned features, direct imperative requests, with the 3 softeners found, are also frequently used among the forms selected by correspondents. The findings of this study, noticeably, point towards an adoption of directness when an action is needed, independently if it is the first time interchanging information between addresses. Thus, concerning requests for action it is shown that performatives and imperatives are the most commonly used; therefore, this suggests that the senders have enough clarity of what they need. In contrast, when requesters need some information, softeners are written to mitigate the imposing effect. Hence, when the questions are performed with a softener, it insinuates that participants waver in their request.

The results of the second taxonomy, conventionally indirect (hearer - based) show considerable variation in its use compared with the direct request. More formal requests are performed in this 
parameter, however, only ability questions are employed by both participants. Moreover, Participant 1 uses sometimes suggestory formulae, and Participant 2 permission request. The findings reported above seem to suggest that requests for information are written more politely since the knowledge needed in that step is not clear at all for correspondents. Thus, imposition is avoided in order to achieve as much information as possible to clarify the process.

At first glance, it seems that conventionally indirect (speakerbased) and indirect requests are not much practiced along correspondence since Participant 1 only uses 1 conventionally indirect (speaker-based) and 4 indirect questions; and Participant 2 uses one of each of the previously mentioned categories. A possibility that appears more plausible for the present results seem to be the misunderstanding an indirect request can cause, since meaning can differ depending on the reader perspective. When arranging a shipment it must be straight and precise as a mistake means an extra cost in the transaction. As a result, it can be important to avoid indirectness either to make requests for action or information, since it can be confusing and imprecise. Ergo, being too much polite might disturb the shipment flow.

Using more direct than indirect strategies does not mean that Vietnamese people are impolite in their requests, but straight up to the point they need to know. As a whole, it can be discussed that requests for action are created with performatives and imperatives which go directly to the point as participants have in mind what to order. Whereas requests for information are more polite and indirect since they need clarification and all knowledge is not at their side. In conclusion, in order to be more fluent when arranging all the exportation and importation process, both the sender and receiver must master the steps needed. Hence, indirectness should be avoided and polite directness used to achieve a good cooperation.

Referring to the limitations of the present study it is important to mention that only 44 emails have been analysed. As a consequence, it is mostly Participant 1 that intervenes. Age, cultural background, and gender have not been considered. Therefore, although the current study is based on a small sample, the findings suggest the importance of studying the phenomenon further to introduce all variables in order to broaden the results discussed.

\section{References}

Alzeebaree, Yaseen, and Mehmet Ali Yavuz. 2017. «Realization of the Speech Acts of Request and Apology by Middle Eastern EFL Learners.» 13 (11): 7313-7327. doi: 10.12973/ejmste/79603. 
Bhatia, Vijay K., and Stephen Bremner. 2012. «English for Business Communication.» Language Teaching 45 (4): 410-445. doi: 10.1017/S0261444812000171.

Biesenbach-Lucas, Sigrun. 2007. "Students writing emails to faculty: An exmination of email politeness among native and non-native speakers of English.» Language Learning \& Technology 11 (2): 59-81.

Bocanegra-Valle, Ana. 2015. «Intercultural Learners, Intercultural Brokers and ESP Classrooms: The Case of a Shipping Business Course.» Procedia - Social and Behavioral Sciences 173: 106-112. doi: 10.1016/j.sbspro.2015.02.038.

Bocanegra-Valle, Ana. 2012. "Maritime English.» In The Encyclopedia of Applied Linguistics, edited by Carol A. Chapelle, 3570-3583. Oxford: Wiley-Blackwell.

Carrió Pastor, María Luisa, and Rut Muñiz Calderón. 2012. «Lexical variations in business e-mails written by non-native speakers of English.» LSP Journal 3 (1): 4-13.

Cenoz, Jasone, and Jose F. Valencia. 1996. "Cross-Cultural Communication and Interlanguage Pragmatics: American vs. European Requests." In Pragmatics and Language Learning, edited by Lawrence F. Bouton, 47-53. Urbana: University of Illinois.

Demydenko, Nadiya. 2012. "Teaching Maritime English: A Linguistic Approach.» Journal of Shipping and Ocean Engineering 2: 249254.

Duthler, Kirk W. 2006. "The Politeness of Requests Made Via Email and Voicemail: Support for the Hyperpersonal Model.» 11 (2): 500-521. doi: 10.1111/j.1083-6101.2006.00024.x.

García Mayo, María del Pilar. 2000. English for specific purposes: discourse analysis and course design. Bilbao: Universidad del País Vasco.

Grice, H. Paul. 1979. "Logic and conversation.» In Syntax and Semantics 3: Speech Acts, edited by Cole Peter and Jerry Morgan, 41-58. New York: Academic Press.

Hyland, Ken. 2007. «English for Specific Purposes.» In International Handbook of English Language Teaching, edited by Jim Cummins and Chris Davison, 391-402. Boston, MA: Springer US.

Martínez-Flor, Alicia, and Esther Usó-Juan. 2010a. «Pragmatics and speech act performance.» In Speech Act Performance: Theoretical, empirical and methodological issues, edited by Alicia Martínez-Flor and Esther Usó-Juan, 3-20. Amsterdam: John Benjamins. 
Martínez-Flor, Alicia, and Esther Usó-Juan. 2010b. «Requests: A sociopragmatic approach." In Speech Act Performance: Theoretical, empirical and methodological issues, edited by Alicia Martínez-Flor and Esther Usó-Juan, 237-256. Amsterdam: John Benjamins.

Mousavi, Seyed Iman. 2012. "Contrastive Rhetoric: Investigating Politeness and Intimacy in Business Email Communications in Four Asian Countries.» The International Journal of Humanities 19 (1): 85-100.

Pettersson, Lena. 2015. "Writing Business Emails in English as a Lingua Franca - how informal can you be?» BA BA, English LInguistics, Stockholms Universitet.

Robinson, Sarah. 2013. "Seafarer Training. Are we all on the same page?» Telegraph, 28-29.

Salvesen, Kristine Elisabeth. 2015. "Politeness strategies in requests by Norwegian learners of English in comparison with native English speakers." Hawaii Pacific University TESOL Working Paper Series 13: 53-69.

Schauer, Gila A. 2009. Interlanguage Pragmatic development. The study Abroad Context. London: Continuum.

Trosborg, Anna. 1995. Interlanguage Pragmatics. Requests, Complaints and Apologies. Berlin: Mouton de Gruyter.

Zarepour, Fatemeh, and Masoumeh Imani Saidloo. 2016. "An Analysis of Iranian EFL Learners' English Written Requestive Emails." Journal of Language Teaching and Research 7 (3). doi: 10.17507/jltr.0703.20. 


\section{Teaching and learning English vocabulary in a Spanish context}

Andreea Stefanescu al310593@uji.es 


\section{Abstract}

\section{(Abstract}

Although the problem of teaching and learning vocabulary has experienced a great development in the last decades, in the contemporary context, teaching vocabulary knowledge is still a major challenge on all levels. The present article attempts to tackle and solve some of the issues encountered by both teachers and students in the classroom regarding vocabulary knowledge. This article presents a study examining the effects of the Vocabulary Box, Kick Me Activity, and the Vocabulary Survey in $2^{\text {nd }}$-year $\mathrm{ESO}^{1}$ students, to show if the method of learning words has an impact on the student learning process. Results are based on the final written test, student observation, and student survey. Different methods produced significant variations in achievement scores, and the data-collection instruments showed that students had a preference for the Vocabulary Box and selected the Kick Me Activity as a second choice. This empirical study produces findings linked to the interaction of students with different dynamic and stimulating tasks that encourage not only participation, fun and engagement, but also vocabulary knowledge acquisition.

Keywords: English vocabulary, Spanish context, learning.

\section{Introduction}

The end of the last millennium has seen a resurgence of interest in the teaching of foreign languages and, in particular, vocabulary. Societal changes and the progress of modern science are reflected primarily and directly through vocabulary. This research presents several questions that need to be addressed when thinking about teaching and learning English vocabulary: Why is it important to teach vocabulary? What is the best way to teach vocabulary? What kind of tools can teachers implement in teaching vocabulary?

\section{Objectives}

The main objective of this article is to identify students' perceptions on vocabulary using a survey (table 1). The second objective is to implement new, dynamic and interactive tools for teaching English vocabulary in a Spanish context. These vocabulary tools are intended to help build communication and interaction in the classroom. A specific objective of this research is to assess their

\footnotetext{
${ }^{1}$ Compulsory Secondary Education
} 
efficiency and, also, to look for alternatives, in case they do not achieve the target.

\section{Material and method}

An eminently practical and dynamic methodology is applied to pre-reading and post-reading stages. Students, as protagonists in the construction of their vocabulary learning, must participate actively and cooperatively in order to facilitate meaningful learning in coexistence with others. The methodological strategies used will be: the Vocabulary Box game as an icebreaker and the Kick Me Activity as the post-reading activity.

Each of the necessary materials and didactic resources to carry out the activities programmed throughout the vocabulary games are shown. Classroom requirements as well as the necessary material are detailed.

\begin{tabular}{ll}
\hline $\begin{array}{l}\text { Classroom } \\
\text { requirements: }\end{array}$ & $\begin{array}{l}\text { language laboratory with good acoustic conditions, } \\
\text { computer and internet connection, blackboard, } \\
\text { projector and projection screen. }\end{array}$ \\
\hline $\begin{array}{l}\text { Materials required } \\
\text { by the students: }\end{array}$ & pens, paper, handouts \\
$\begin{array}{l}\text { Methodological } \\
\text { aspects: }\end{array}$ & $\begin{array}{l}\text { communicative approach, cooperative learning and } \\
\text { interaction }\end{array}$ \\
\hline
\end{tabular}

\section{Vocabulary Survey}

To begin with, a structured survey was undertaken to identify what students thought about learning English vocabulary in general. In order to collect evidence from many students, a survey tool was created (Table 1). A relatively large sample group of 79 students was gathered to provide a range of results. The survey was conducted in IES Bovalar with $75 \%$ students who speak Spanish as L1, and $25 \%$ students who are immigrants. This questionnaire sought to identify what students think about English Vocabulary in general, and how it can be learned in a Spanish high school context. 


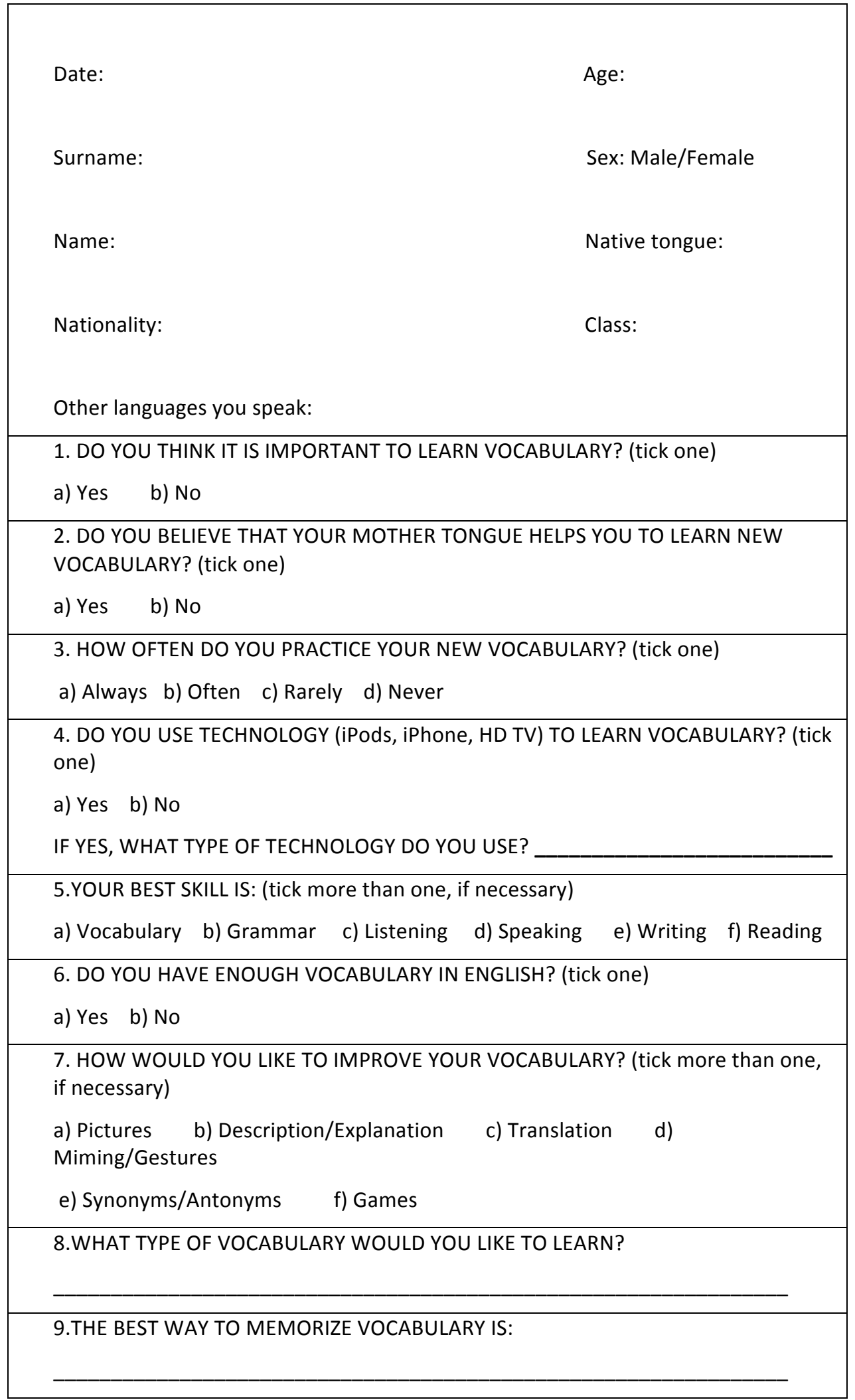

The assumption was made that students from different levels ( 2 $\mathrm{ESO}^{2}, 4 \mathrm{ESO}$, and 1 BACHILLERATO) would together constitute a

\footnotetext{
${ }^{2}$ Compulsory Secondary Education
} 
representative sample of pupils as a whole. The study was carried out in the following period: $27 / 04 / 2015-30 / 04 / 2015$. The groups that were investigated included boys and girls between the ages of 13-18. Therefore, the survey provided data on general vocabulary knowledge and acquisition. We have chosen only a few questions from the Survey to analyse those that we considered as more relevant to our research:

Table 2. Students percentage on some relevant questions

\begin{tabular}{|l|l|}
\hline \multicolumn{1}{|c|}{ Survey Questions } & \multicolumn{1}{|c|}{ Student percentage } \\
\hline $\begin{array}{l}\text { 1. Do you think it is important } \\
\text { to learn vocabulary? }\end{array}$ & $\begin{array}{l}\text { YES: } 99 \% \\
\text { NO: } 1 \%\end{array}$ \\
\hline $\begin{array}{l}\text { 2. Do you believe that your } \\
\text { mother tongue helps you to } \\
\text { learn new vocabulary? }\end{array}$ & $\begin{array}{l}\text { YES: } 59 \% \\
\text { NO: } 41 \%\end{array}$ \\
\hline $\begin{array}{l}\text { 4. Do you use technology (iPods, } \\
\text { iPhone, HD TV) to learn vocabulary? } \\
\text { (tick one) }\end{array}$ & YES: $71 \%$ \\
\hline $\begin{array}{l}\text { 6. Do } 29 \% \\
\text { vocabulary in English? }\end{array}$ & YES: $52 \%$ \\
\hline
\end{tabular}

Regarding the first question, the acquisition of vocabulary in language learning is as essential as numbers are in mathematics. Furthermore, looking at the percentage rate ( $99 \%$ for yes), it is evident that the relevance of vocabulary for pupils is very high. Moreover, the effort of the teachers to cultivate in students a taste for new vocabulary is greater, and it is reflected in the survey answers that students are aware of the importance of learning new vocabulary.

Regarding the second question on the survey, Schmitt and McCarthy stated that: "recent research has confirmed more and more strongly what language teachers have always known: that the mother tongue has a considerable influence on how the second language is learnt and used» $(1997,160)$. Therefore, the influence of the mother tongue is very important when learning vocabulary in another language, as Schmitt and McCarthy state: "the mother tongue can influence the way second-language vocabulary is learnt, the way it is recalled for use, and the way learners compensate for lack of knowledge by attempting to construct complex lexical items». (1997, 179).

The fourth question highlights that vocabulary knowledge is acquired with the help of new technology, such as computers, iPhones, iPads and iPods. The findings in Table 2 show that a majority 
of students (71\%) use electronic devices to learn new vocabulary; however, only $29 \%$ of students prefer other materials to improve their vocabulary. Electronic devices brought a vast range of possibilities to the educational plan, particularly in the field of learning vocabulary. Some of the benefits of those devices are to give students instant feedback, portability, and fun. Therefore, using electronic devices is a convenient way for students to practice and learn new vocabulary.

Finally, the sixth question explores the students' opinion regarding the amount of English vocabulary that they possess. Some of them were optimistic by saying "yes", they had a wide range of vocabulary, while others seemed more realistic and responded with a «no».

Table 3. Students percentage on question seven from the Survey

\begin{tabular}{|l|l|}
\hline Survey Questions & \\
& Multiple responses \\
\hline 7. HOW WOULD YOU LIKE TO & a) Pictures: $35 \%$ \\
IMPROVE YOUR VOCABULARY? & b) Description/Explanation: $14 \%$ \\
(tick more than one, if necessary) & $\begin{array}{l}\text { c) Translation: } 39 \% \\
\text { d) Miming/Gestures: } 14 \%\end{array}$ \\
& $\begin{array}{l}\text { e) Synonyms/Antonyms: } 8 \% \\
\text { f) Games: } 56 \%\end{array}$ \\
\hline
\end{tabular}

When it comes to the strategies column, the results in Table 3 show that more than half of the respondents -56 out of 79 students - think they can improve their English vocabulary with the help of games. This can, at least partially, be attributable to the study style encouraged by their English teacher, Alicia Garcia Martí, who implemented different games and strategies that foster the students' motivation.

As for the use of translation and pictures, it was found that a considerable number of students -39 and 35 subjects - try to use them to enhance the new vocabulary. The least preferred strategy to improve vocabulary was through synonyms and antonyms, to which only 8 students responded.

Hence, results in Table 3 show that students seem to prefer learning vocabulary through games and translation. Knowing the famous proverb that "A picture is worth thousand words", teachers should consider and apply this reflection in their teaching. However, it should be indicated that, from the perspective of students, very important causes that lead to learning new vocabulary are linked to both teachers and the students' performance.

In conclusion, it is important to mention that the present survey has identified and contributed to the discovery of the opinions and 
attitudes towards vocabulary knowledge in a Spanish context. We can conclude that these are all strategies which students already use and regard as beneficial. The fact that students prefer games leads us to the next step.

\section{Games}

Society is in the process of developing communication, of enriching vocabulary with new words. Humans invented crosswords, scrabble, etc.; hence, we can say that words have become a type of "toy» for individuals. People use them whenever and however they want. Unlike traditional methods of teaching foreign language vocabulary, modern methods involve interactivity and a direct participation of those trained in the teaching-learning process. One of the most useful interactive methods for learning a modern language and, particularly new vocabulary, is the incorporation of games: "to memory and performance benefits, games and interactive learning methods have important social benefits for students. These activities allow students to practice using the vocabulary of the discipline» (Kumar Rita and Robin Lightner 2007).

Before explaining the impact of games in teaching vocabulary, it is relevant to clarify the meaning of the word game. According to the Oxford Dictionary, the concept game means: "a form of competitive activity or sport played according to rules». The present section focuses on a number of advantages that students can experience with games while also learning new vocabulary.

As mentioned above, games have multiple advantages for students, especially when they learn new vocabulary in English. Firstly, games have the effect of motivating pupils. In this respect, Ali Sorayaie indicated that: "games can lower anxiety, thus making the acquisition of input more likely. They are highly motivating and entertaining, and they can give shy students more opportunity to express their opinion and feelings» $(2012,253)$. Therefore, games have the power of motivating and challenging students in an exciting manner, and sometimes even the timidest students feel free and relaxed (this happened during our research).

Secondly, Byrne points out that games can be performed at all stages of learning, by reviewing and acquiring new vocabulary:

The maximum benefit can only be obtained from language games if they form an integral part of the programme, at both the practice and the production stages of learning. Used in this way, they provide new and interesting contexts for practicing language already learnt- and often for acquiring new language in the process $(1976,99)$. 
Thirdly, according to Harmer, games are one of the teacher's tools that can lead to the assimilation of words much easier, creating a relaxed learning environment:

Games are a vital part of a teacher's equipment, not only for the language practice they provide, but also for the therapeutic effect they have. They can be used at any stage of a class to provide an amusing and challenging respite from other classrooms activity, and are especially useful at the end of a long day to send the students away feeling cheerful about their English class $(1983,94)$.

In the same line, Thornbury states that: "useful games are those that encourage learners to recall words and, preferably, at speed» (2002, 102).

Another aspect that must be mentioned in this section is that the teaching of games at high-school level is different from primary one. Although it seems harder to involve students in these activities, they are interested, as it breaks down the barriers and represents a deviation from their usual class activities. In this case the didactic game can be used to, either start a discussion, in order to «break the ice», to highlight competitive features of participants, or to review some concepts studied.

However, if the games are not well explained by the teacher, they cannot be played well and achieve the proposed goals. In this regard, Thanh Huyen and Thu Nga declare that: "games cannot be successful if the teacher does not explain the tasks and roles of students clearly in playing games» $(2003,12)$.

Many researches argue that games have a powerful impact on students; however, teachers must take into consideration different aspects when they implement games in the class. For instance, Thornbury states that: «many word games deal solely with isolated rather than contextualized- words, and often require only shallow processing on the part of the learner, they should be used judiciously» $(2002,102)$. Thus, efficiency depends on the game and what it actually fosters in a class.

To meet the specific objective, we tried to implement two types of vocabulary games, in order to gain information regarding the students' satisfaction and acquisition of new vocabulary. Below we will list two types of games and when they can be used as part of a lesson. Games can be used at the beginning of the class, in the middle and at the end. 


\section{Procedure}

This game can be used for all levels and ages. The game should last no longer than five to ten minutes. This warm-up game can be applied at the beginning of a class. The teacher asks every student to pick a word from the box and then translate it into Spanish. At the end of the class, the teacher provides students with a piece of paper onto which they write their newly acquired word which they then put it into their so-called "Vocabulary Box».

We did this activity for a month during my internship at IES Bovalar at the beginning and the end of our classes with the three different groups that we taught. What we found interesting was the fact that one student wrote the same word five times on the piece of paper to, perhaps, retain the word, whereas another student returned a blank piece of paper, arguing that he had not learnt anything in the lesson. However, what really surprised us was the students' attitude towards this activity. One day when we forgot to offer them the game, they reminded us by saying: "When are we doing the Vocabulary Box?». This activity can be used as a warm-up to check what students remember from the previous lesson.

Therefore, we can say that the Vocabulary Box is a type of "Pandora's box», as it arouses students' curiosity, mixed with other feelings such as: anticipation, joy, intuition, and creativity. In the last class with our students, we wanted to surprise and reward them for their participation, interest, enthusiasm, and for creating a good atmosphere, by putting sweets into the Vocabulary Box instead of words. We wanted to thank them in a special way as the experience had been very rewarding for us.

\section{Study case of the «Kick me Activity»}

\section{Methodology and Procedures Participants of the "Kick me Activity»}

This game can be implemented at all levels and at any age. As this research was based on a specific task called "Kick Me Activity", we chose three different classes of the same level (A B; C, E; and D), in IES Bovalar, Castelló de la Plana, Spain. There were 43 students between the ages of 11 and 15, and their level of English can be classified from elementary to intermediate.

The three groups were chosen because we considered the task adequate for their level. Moreover, they were some of the groups that had been taught by their tutor. For this reason, it can be said that they had almost the same level and interests. 
The first group ( $2^{\text {nd }}$ ESO A, B) consisted of 16 students. When we introduced the activity, there were only 10 students ( 6 boys and 4 girls). The tutor commented that in this course the students had a lower level of English than in the other two groups. Students gave the impression that they were not very interested in English. The second group ( $2^{\text {nd }}$ ESO C, E) was made up of 15 students; however, only 12 students ( 2 boys and 10 girls) were present on the day we performed the task. Even though this class had more reluctant students regarding the subject, it worked better than with the first group, as they enjoyed doing the task.

The third group ( $2^{\text {nd }}$ ESO D) consisted of 20 students with 17 students (11 boys and 6 girls) present on the day of the activity. Despite being a larger group $t$, the expectation was higher, as their level of English was better and their interests very well defined. Most of the students tried their best using English as their vehicular language during this activity. Furthermore, their tutor mentioned that this group was more responsible and engaged in the English Subject.

The objectives of the Kick Me Activity are: this game is used in the post-reading stage, to learn new vocabulary, students have to match the words with their exact definition and it can be implemented in any subject. The Kick Me activity was developed in the post-reading stage, and it encourages movement in the class, increasing focus, engagement, and retention of information. The teacher gives the students a handout where there are some definitions on the right side of the paper, and they have to find the missing words on the left side for those definitions (Table 4.). Therefore, students have to find the answers to blanks on the worksheet by looking at the words that the teacher put on the students' backs, using labels. As a result, throughout this activity, students learn the new vocabulary that is going to be in the text. In this way, during the while-reading stage, they know the meaning of the new words that appear in the text. 
Table 4. Student's Handout

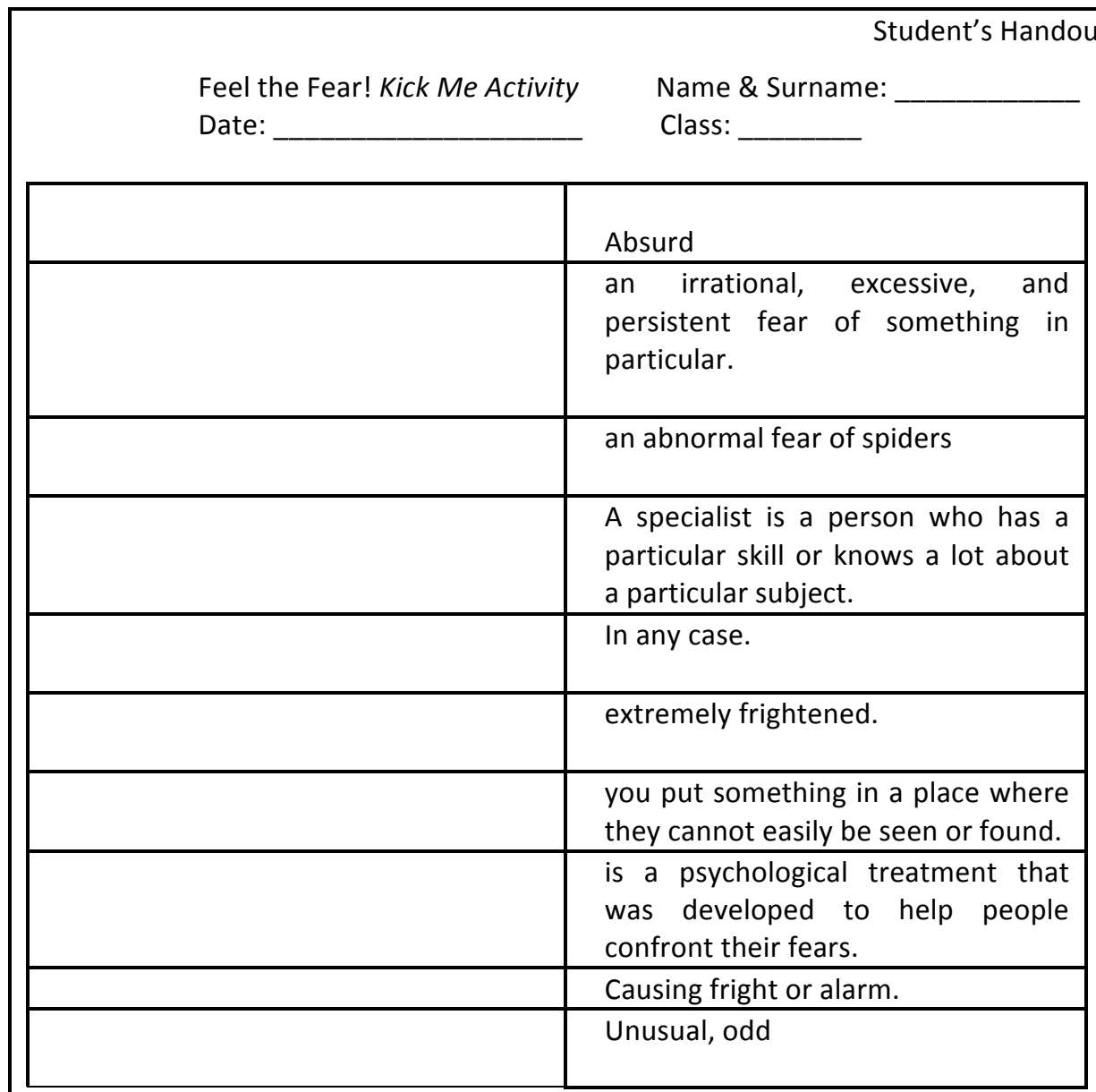

This activity consists of the following rules:

- Nobody gets kicked during this activity!

- Find the answers for your worksheet on the backs of your classmates.

- When you have them all, sit down at your desk, so that others can still see your label. 
Table 5. Teacher's Handout

\begin{tabular}{|c|c|}
\hline \multirow[b]{2}{*}{$\begin{array}{l}\text { Feel the Fear }{ }^{4} ! \text { Kick Me Activity } \\
\text { Date: }\end{array}$} & \multirow{2}{*}{$\begin{array}{l}\text { Teacher's Handout } \\
\text { Name \& Surname: } \\
\text { Class: }\end{array}$} \\
\hline & \\
\hline Ridiculous & \multirow{3}{*}{$\begin{array}{l}\text { Absurd } \\
\text { an irrational, excessive, and } \\
\text { persistent fear of something in } \\
\text { particular. }\end{array}$} \\
\hline & \\
\hline Phobia & \\
\hline Arachnophobia & an abnormal fear of spiders \\
\hline Specialist & $\begin{array}{l}\text { A specialist is a person who has a } \\
\text { particular skill or knows a lot about } \\
\text { a particular subject. }\end{array}$ \\
\hline Anyway & In any case. \\
\hline Terrified & extremely frightened. \\
\hline Hide (Hid) & $\begin{array}{l}\text { you put something in a place where } \\
\text { they cannot easily be seen or found. }\end{array}$ \\
\hline Exposure Therapy & $\begin{array}{l}\text { is a psychological treatment that } \\
\text { was developed to help people } \\
\text { confront their fears. }\end{array}$ \\
\hline Scary & Causing fright or alarm. \\
\hline Strange & Unusual, odd \\
\hline
\end{tabular}

The teacher gives the students ten minutes to finish their task matching 10 words with their appropriate definition (Table 4.). When the time finishes, the teacher says: "Time is up! ", and the students have to sit down. After that, the teacher checks out aloud who did it right, and corrects the errors. When implementing this activity, teachers have to take into account several criteria such as:

- Considering not only the students' level of English but other factors too, such as: motivation, amount of previous knowledge, social situation, geographic location, cultural level, genetic features (some have an innate intelligence - predisposition to learn new languages), age, etc.

- Time is a crucial factor. This activity cannot last more than ten minutes, as the students lose concentration, interest, etc.

- Number of students (amount). Within a large class the activity works better (15- 30 students).

- The classroom environment in general - affective relationship that is established between teacher and students, and the peer relationship.

\footnotetext{
${ }^{4}$ The didactic Unit that we were working that time, according to the text book was called Feel the Fear- that is the reason of why using these handouts.
} 


\section{Results}

To carry out this research we focused on the understanding and the attitudes of students, and what they actually learnt during this activity. To observe what positive and negative outcomes come from the adoption of a game in vocabulary acquisition, it is essential to examine the following factors: the students' responses, engagement, and achievement.

Table 6. Student reaction

\begin{tabular}{|l|}
\hline Student reaction \\
\\
\\
\hline Class A, B: $40 \%$ \\
Class C, E: $73 \%$ \\
Class D: $88 \%$ \\
\hline
\end{tabular}

Regarding the students' reactions towards the activity, there were different types of responses depending on their class, mood and interests. Firstly, in the classes C, E, we observed that their reactions were quite normal, even though they seemed somewhat excited at the beginning of the activity. Secondly, in class A, B, students were not enthusiastic at all, perhaps because they were not interested in the theme, or perhaps they felt somewhat lethargic (it was Friday). Thirdly, in class $D$, most of the learners (15 out of 17) were very interested and curious about "Kick Me Activity» and tried their best. They seemed to be perseverant with finding the answers while a relaxed atmosphere fostered the competitiveness in the classroom.

Table 7. Students' participation

\begin{tabular}{|l|}
\hline \multicolumn{1}{|c|}{ Students' participation } \\
\hline $\begin{array}{l}\text { Class A, B: } 50 \% \\
\text { Class C, E: } 87 \% \\
\text { Class D: } 94 \%\end{array}$ \\
\hline
\end{tabular}

In this part of the study, it is important to consider the students' positive or negative participation in the activity. This task provided an alternative to represent the students' level of engagement, ontask response and peer interaction. For example, these responses could be reproaches regarding the activity, or positive remarks about doing similar activities in other classes. We calculated the percentage of their positive and negative comments regarding the task. The result was that in class $D$ most of the research students $(70 \%)$ 
thought that during the task they participated more, as they did not feel any pressure from the teacher side. We would say that the students actively engaged in this activity, even the shy ones.

By contrast, in class A, B, some of the students did not participate the way we expected. They tended to speak in Spanish about unrelated topics. For example, when we were approaching some of them, they were talking about soccer. Additionally, in class C, E, students had a tendency to take more risks than in other classes, which, in a sense, helped them perform the task and be more participative.

We would say that, based on the research data, the students participation was overall good. We realized that we had more interaction with the students on the days that we did these types of activities, which may indicate their preferences for these types of tasks. What really surprised us was the participation of a student, who during the observation period was very distracted; however, in the end, this student's response to the task was amazing and collaborative.

Table 8. Student achievement

\begin{tabular}{|l|}
\hline \multicolumn{1}{|c|}{ Student achievement } \\
\hline \\
\hline $\begin{array}{l}\text { Class A, B: } 60 \% \\
\text { Class C, E: } 80 \% \\
\text { Class D: } 88 \%\end{array}$ \\
\hline
\end{tabular}

Regarding the students' accomplishment, the "Kick Me Activity», apart from providing fun, a relaxed atmosphere and curiosity, also developed the academic performance, and this was reflected in the final written exam. In this exam, there were different sections; however, we would say that around $85 \%$ of students were successful in the vocabulary section.

To collect results, both quantitatively and qualitatively, we worked with the students' survey answers, and we kept an observation journal to monitor their involvement in vocabulary activities. In the journal, we recorded various comments made by the students regarding the "Kick Me Activity». For example: «Me gustó mucho el juego», "me encantó», "¿lo podemos hacer de nuevo?». During the days that followed they kept asking us if we were going to do it again.

\section{Discussion and conclusions}

Throughout this activity, students interacted with each other, and also learnt new words from each other. Regarding the 
effectiveness of "Kick Me Activity", we can report that students seemed to learn new vocabulary. This game encourages vocabulary development and free expression, but it can also be used for vocabulary revision at the end of a unit.

This method is well suited for students with a lowerintermediate to advanced level, because they can formulate more extensive definitions; hence, helping themselves with their accumulated vocabulary. It is important to take into consideration that the study we made was limited as to the student groups, age, and time factors.

In summary, the "Kick Me Activity» makes the process of assimilation of new words more interactive and fun. Therefore, this task has become a tool of communication and discovery, and has created a more dynamic and participative atmosphere, as the activity is challenging and encourages the students' intuition and teamwork. However, the "Kick Me Activity" also presents several disadvantages; for instance, students might speak among each other about other things in their mother tongue, which creates minor chaos in the class.

In consequence, «increasing vocabulary knowledge is a basic part of the education process, both, as a means and as an end", declared the American linguist Nagy William. Vocabulary knowledge is achieved not only at school, but at home, by different means, such as watching movies or English programs, by interaction with native people, etc. Hence, there are many factors that foster the improvement of vocabulary. The present study tried to find and solve some of the problems that teachers and students face in the classroom regarding vocabulary knowledge.

It can be said that, according to these results, interaction, dynamic, cooperative games are the best way to teach and improve vocabulary in a Spanish context. This research showed the effects of learning tools, such as the Vocabulary Box, Kick Me Activity, and the Vocabulary Survey on 2 ESO students, with the objective of finding out whether the method of learning new words influenced the students' learning process. These procedures led to significant changes in achievement scores, and the data collection instruments showed that students selected the Vocabulary Box as their first choice, and the Kick Me Activity as a second option. The results were obtained by using the final written test, through the observation of students, and the survey taken.

Gunn Tim claimed that: "Few activities are as delightful as learning new vocabulary». This empirical research points out a set of interesting findings, of which the most relevant are related to the interaction of students with different dynamic and stimulating tasks, that encourage not only collaboration, entertainment and responsibility, but also the knowledge of vocabulary. 
VII. References

American Psycological Association. 2015. "What is Exposure Therapy?». Accessed June 1, 2015. https://www.apa.org/ptsdguideline/patients-and-families/exposure-therapy.

Amy, Bailey, Gina and Lori. 2012. "Let's Talk About Vocabulary Strategies». Concord, NC: Cox Mill High School. Accessed on May 10 , 2015 , https://literacy.uncc.edu/sites/literacy.uncc.edu/files/media/do cs/Let's\%20Talk\%20About\%20Vocabulary\%20Strategies\%20pres entation.pptx.

Byrne, Donn. 1976. Teaching Oral English. Longman Print. Essex.

Collins Dictionary, s.v. «anyway», accessed June 1, 2015, https://www.collinsdictionary.com/es/diccionario/ingles/anywa $\mathrm{y}$.

Collins Dictionary, s.v. "arachnophobia», accessed June 1, 2015, https://www.collinsdictionary.com/dictionary/english/arachnop hobia.

Collins Dictionary, s.v. «hide», accessed June 1, 2015, https://www.collinsdictionary.com/dictionary/english/hide.

Collins Dictionary, s.v. «phobia», accessed June 1, 2015, https://www.collinsdictionary.com/dictionary/english/phobia_1.

Collins Dictionary, s.v. «ridiculous», accessed June 1, 2015, https://www.collinsdictionary.com/dictionary/english/ridiculous

Collins Dictionary, s.v. "scary», accessed June 1, 2015, https://www.collinsdictionary.com/es/diccionario/ingles/scary.

Collins Dictionary, s.v. "specialist», accessed June 1, 2015, https://www.collinsdictionary.com/dictionary/english/specialist.

Collins Dictionary, s.v. "terrified», accessed June 1, 2015, https://www.collinsdictionary.com/dictionary/english/terrified.

Gunn, Tim. 2007. A Guide to Quality, Taste \& Style. New York: Abrams Image.

Harmer, Jeremy. 1983 The Practice of English Language Teaching. Essex: Longman Print.

Kumar, Rita and Robin Lightner. 2007. "Games as an Interactive Classroom technique: Perceptions of Corporate Trainers, College Instructors and Students». International Journal of Teaching and Learning in Higher Education 19 (1): 53-63. 
Mackay, Molly. 2007. Vocabulary Development. Activities and Games for Early Learners. Hunington Beach: Teacher Created Materials.

Merriam-Webster Dictionary, n.d. "a picture is worth a thousand words», accessed May 27, 2015, https://www.merriamwebster.com/dictionary/a\%20picture\%20is\%20worth\%20a\%20t housand\%20words.

Nagy, William. 1988. Teaching Vocabulary to Improve Reading Comprehension. Newmark: International Reading Association.

Oxford Dictionary, n.d. "game», accessed May 20, 2015, https://en.oxforddictionaries.com/definition/game.

Schmitt, Norbert and Michael McCarthy. 1997. Vocabulary: Description, Acquisition and Pedagogy. Cambridge, UK: Cambridge University Press.

Sorayaie, Ali. 2012. "The Effect of Games on EFL Learners' Vocabulary Learning Strategies». International Journal of Basic and Applied Science 1 (2): 252-256.

Thanh Huyen, Nguyen Thi and Khuat Thi Thu Nga. 2003. «Learning Vocabulary Through Games: The Effectiveness of Learning Vocabulary Through Games». The Asian EFL Journal 5 (4): 1-15.

Thornbury, Scott. 2002. How to Teach Vocabulary. Essex: Longman Print.

Walters State. 2015. "Lesson in a Box: Discipline Specific Vocabulary». Walters State. The Great Smoky Mountain Community College. Accessed on June 11, 2015. http://library.ws.edu/c.php?g=401199\&p=2728615. 

I. Abstract

\begin{abstract}
I. Abstract
Decade after decade language learning still proves difficult for EFL learners all around the world. Countries are consistently having the same phonetic problems they had. This article wants to present two methods commonly used when comparing languages (contrastive analysis and statistics) and add two more, relatively fresh methods (spectrographic analysis and Johari windows), in order to find a new angle to look at old problems. By taking into account more points of view, the resulting data could be a starting point for future researchers facilitating EFL learning for future generations.
\end{abstract}

Keywords: EFL, English vowels, spectrography, statistics, Johari window, contrastive analysis hypothesis.

\title{
II. Introduction
}

When looking through the past history of language learning and its phonetic difficulties, one will find that in the last 100 years not much has changed. Edward Gauntlett, one of the first Linguists studying English pronunciation, while applying modern methods like contrastive analysis, described already in 1914 the same phonetic difficulties Japanese EFL learners still face today (Ashby, and Saito 2013).

This is not an isolated idiosyncrasy, since similar conclusions can be found for most countries that actually bothered to inquire about such matters from almost a century ago. Quite obviously the wheels of research have been spinning in that time, but it seems that they are stuck in the mud, because the best way to summarize the poor progress made in all that time is an even older quote by Goethe: «And here I am, for all my lore, the wretched fool I was before».

This article will follow the philosophy behind my PhD research: trying to deliver an original and interdisciplinary approach at the same time. After all, cognitive linguistics is defined as an interdisciplinary branch of linguistics. And originality is something that is asked of every single PhD student anyway.

\section{Objectives}

This article will thus honor those two key tenets (originality and interdisciplinary) by using both proven linguistic research methods as well as methods used in fields different than linguistics. First, I will describe Contrastive Analysis Hypothesis and Statistics, both very common research methods throughout the years when it comes to comparing languages. Second, I will explain two methods, 
spectrographic analysis (hailing from physics) and Johari windows (a

heuristic technique originating from the psychological field) that when used alongside traditional methods should deliver maybe a look at old problems from a fresh perspective that could benefit future linguists.

\section{Methodology}

I am going to quickly present two methods that have been commonly used in research comparing languages and then provide some further explanation about two methods that, I suggest, should be added to the comparison of languages. First, the contrastive analysis hypothesis (henceforth $\mathrm{CAH}$ ) can be defined as the systematic study of two (or more) languages in order to identify both their structural similarities and differences.

$\mathrm{CAH}$ has been for decades one of the best methods when it comes to language acquisition. It is an effective procedure when you want to compare the speaker's native language (L1) against another language (L2) and how the L1 itself could predictably affect the learning of $\mathrm{L} 2$. Hence $\mathrm{CAH}$ concentrates at the differences and similarities between at least two languages.

This contrastive approach emerged after the end of the Second World War when the US gave importance to foreign language learning. Furthermore, an increasing amount of research based on bilingualism of immigrants was being published.

Professor Robert Lado defined in 1957 the so-called strong version of $\mathrm{CAH}$, which states that all errors made in $\mathrm{L} 2$ were due to interference by $\mathrm{L} 1$ and subsequently all errors could be predicted. No empirical evidence has sustained this claim (Banathy et al. 1966) and it neglected essential criteria such as age. It also focused too much on the inference aspect of the L1 language and offered little use to language teachers (Newmark, and Reibel 1968).

After this strong version, researchers defined an alternative, the so called weak version; this one commences with the errors already made by the learner which then gets explained as much as possible by designating the differences and similarities between both languages (Stockwell 1967). This is viewed as a complementary research to error analysis and intrinsically shares a few of the typical mistakes (e.g. error analysis is not enough when you want to identify learning complications).

Finally, a third (and last) iteration of $\mathrm{CAH}$, a moderate version, came around and became an essential research method for second language acquisition: "the categorization of abstract and concrete patterns according to their perceived similarities and differences is the basis for learning; therefore, wherever patterns are minimally distinct in form or meaning in one or more systems, confusion may 
result» (Oller, and Ziahosseiny 1970). It clearly contradicts Lado's original variant, since the hypothesis here goes the other way round: the more different the $L 2$ is from your own $L 1$, the easier it will be to learn the targeted new language.

\begin{tabular}{|c|c|c|c|c|}
\hline SOUND & \multicolumn{4}{|c|}{ CHARACTERISTICS } \\
\hline \multirow{3}{*}{$/ \mathrm{m} /$} & \multicolumn{2}{|c|}{ Point of Articulation } & \multicolumn{2}{|c|}{ Manner of Articulation } \\
\hline & English & Spanish & English & Spanish \\
\hline & Bilabial & \multirow{2}{*}{$\begin{array}{c}\text { Bilabial (but lips are } \\
\text { contracted without } \\
\text { connection and the tension } \\
\text { is minimal) }\end{array}$} & Nasal & \multirow[b]{2}{*}{$\begin{array}{l}\text { Nasal (air is released } \\
\text { through the nose) }\end{array}$} \\
\hline (voiced) & & & & \\
\hline
\end{tabular}

Figure 1: An Example of $\mathrm{CAH}$

Furthermore, language awareness is a very profitable language learning method for advanced and/or adult learners and it is part of teacher training programmes at many universities (König, and Gast 2009).

The second method is statistics, which is commonly defined as a mathematical branch, which deals with data collection, organization, analysis, interpretation and presentation. It is ergo also known to be a quantitative approach to research when linguistics and languages usually use qualitative methods. These use data exclusively for identifiying language feature usage and then afterwards describing them, while giving real-life examples of specific phenomena (Cantos Gómez 2013).

Supplementing these qualitative analyses of language with the quantitative research method of statistics, can contribute fruitfully to linguistic analysis and research. In the latter, linguistics features are classified and counted to create even more complex statistical models, that explain in more details the observed facts (Cantos Gómez 2013).

Table 1: Example of Consonant vs. Vowel Distribution Chart

\begin{tabular}{|l|l|l|}
\hline & Consonant distribution & Vowel distribution \\
\hline English & $60.78 \%$ & $39.22 \%$ \\
\hline Spanish & $56.51 \%$ & $43,49 \%$ \\
\hline Japanese & $48.00 \%$ & $52.00 \%$ \\
\hline
\end{tabular}

Despite some linguists dismissing quantitative methods, due to lack of training, or even disliking them due to belonging to the realms 
of mathematical sciences, the most popular statistical techniques are also the most accesible ones. In regards to linguistic purposes (Cantos Gómez 2013), they have been used:

1. to observe and describe certain aspects of language phenomena

2. to apply quantitative techniques or model to problems related to describing natural language or language teaching

3. to solve methodological problems of linguistic measurement or sampling

The first new method I propose is spectrography, which is a relatively new approach to linguistics and since it originated in a different field it needs to be explained from the beginning, with the definition of sound.

Sound can be defined as pressure oscillation occurring very rapidly one after another. These fluctuations move through a medium (e.g. gas, water or air) that is elastic enough to allow molecules to crowd together and move apart. In human physiology some of the sounds are called speech. The fluctuations here are caused by the actions (e.g. vibrations) of the vocal organs against the outgoing amount of lung air.

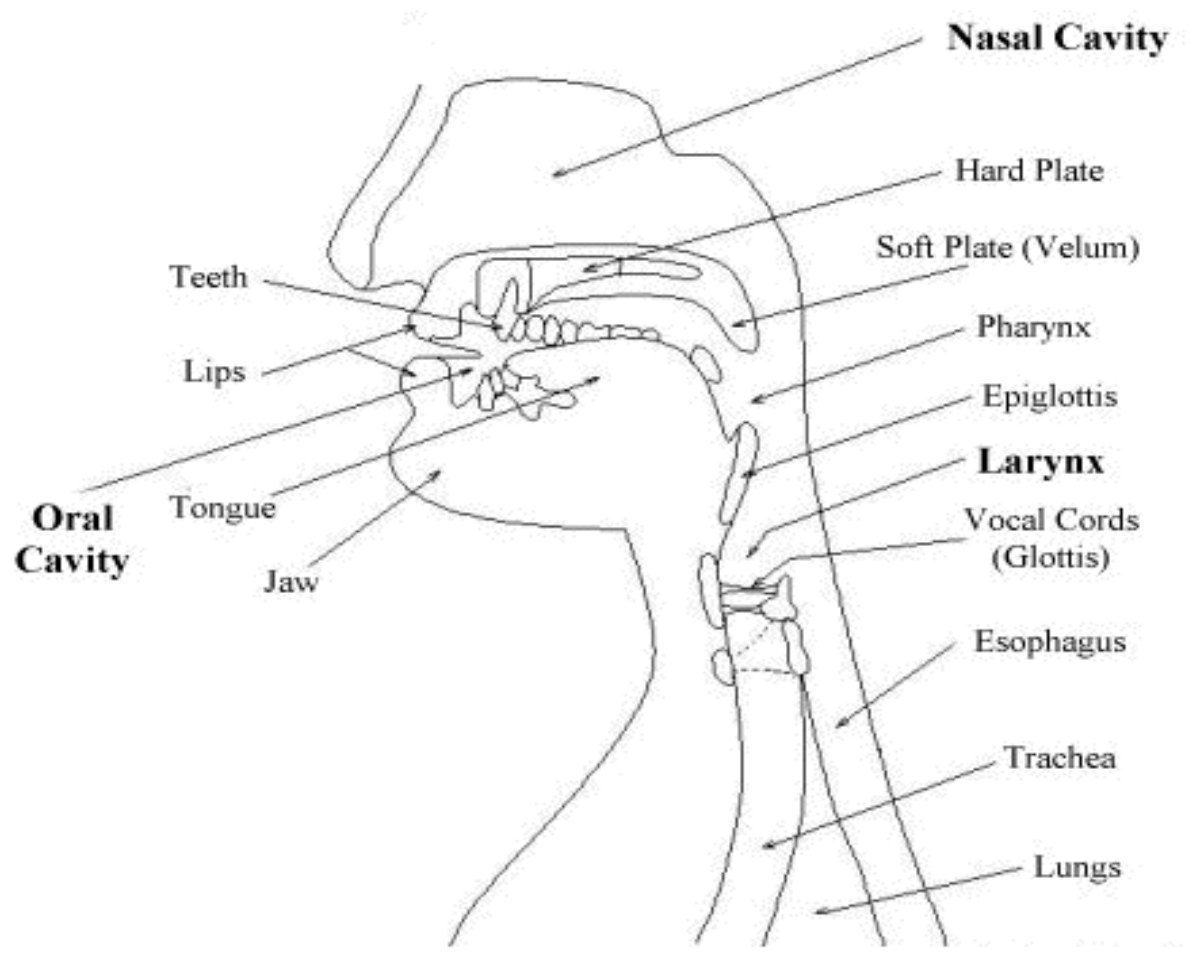

Figure 2: Vocal Organs

The vibrations made in the vocal tract can be visually represented by speech waveforms. These are graphs that visually represent how pressure variation changes with time. 


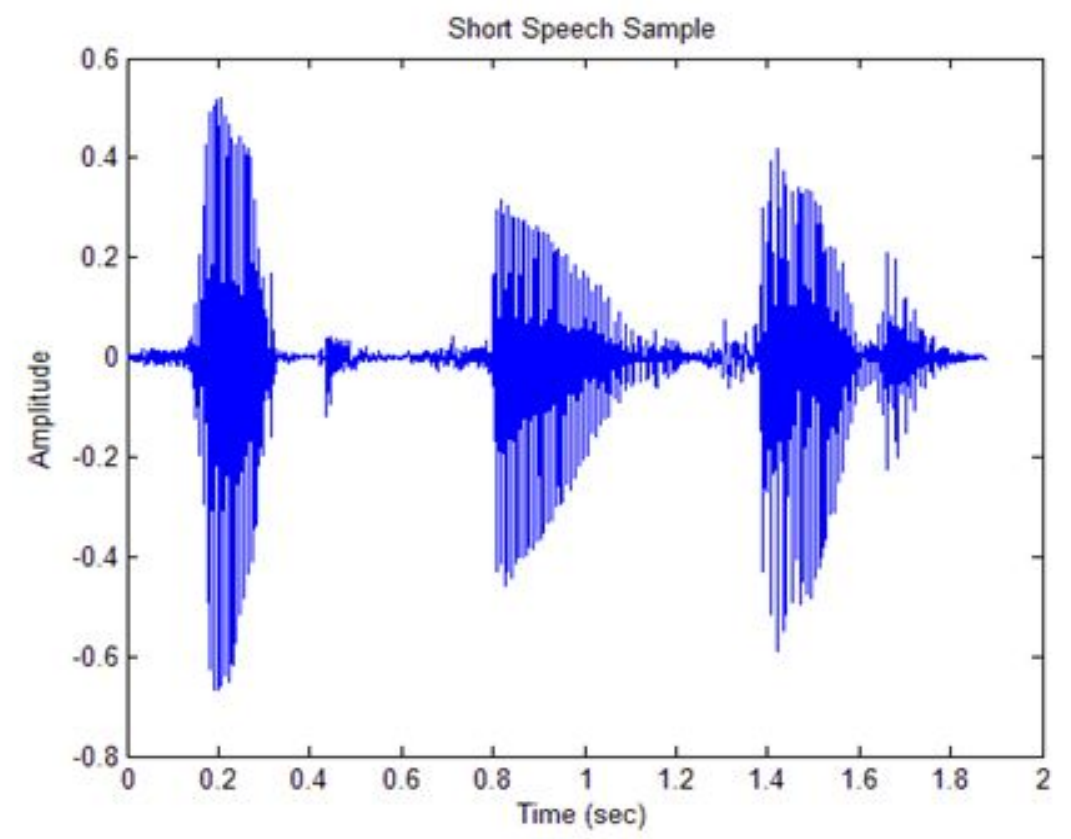

Figure 3: Speech Waveform

The $y$-axis measures the pressure variation (measured as amplitude) while the $x$-axis reflects the time needed for the changes of pressure. The amplitude can be defined as a measure of the average pressure variation from normal atmospheric pressure. Some graphs can show a straight line in the centre of the waveform ( 0 amplitude), which represents normal atmospheric pressure. If the wave is above 0 it means that the pressure is greater than the atmospheric pressure while being below 0 means that pressure is lower than the atmospheric one.

Waveforms have one problem though: it is near impossible to read them as words. Through the use of Fourier analysis we can find out what frequencies are present at any given moment in the speech signal. The result of this analysis can be graphically represented as a spectrum: the amplitude of the sinewave components that are present in the signal within a given time window.

Several spectra though still don't let us identify the sounds in words. But if we compute the spectrum for one short section of speech and keep computing the spectrum for the adjoining short section of speech until the end of the waveform we'll be able to display all the computed spectra in a 3D graph. This graph is called a spectrogram, a visual representation of the frequency and intensity of speech sounds against time. 


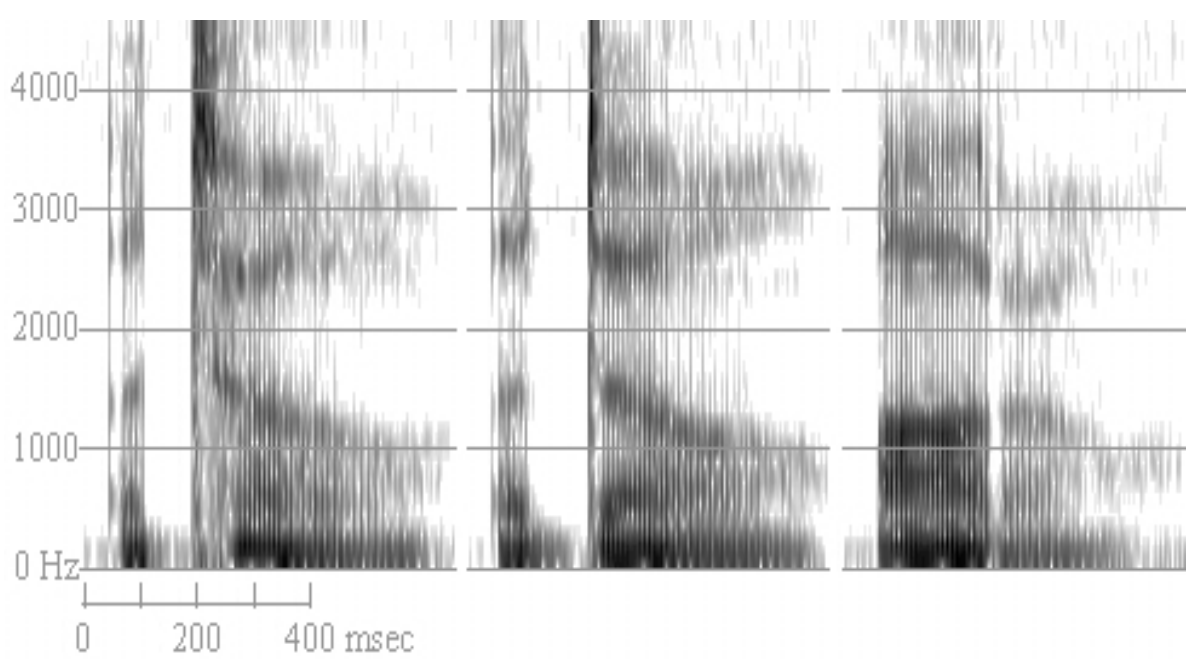

Figure 4: Spectrogram

Spectrograms like picture aboved can be read due to the idiosyncratic phonetic features of phonemes and thus spectrographic analysis can be used to improve language learning (Ladefoged, and Johnson 2011).

The second new method I am adding is the Johari window, which is a heuristic method developed, back in 1955, by psychologists Joseph Luft and Harrington Ingham. Heuristics are techniques that solve problems, learn or discover new information through practical methods though in some cases, due to cognitive biases, can lead to systematic errors. With proper care though, heuristics result in easily accesible information in order to solve problems or even abstract issues (Michaelewicz, and Fogel 2000).

A Johari window, as originally used in psychological contexts, aims to improve interpersonal relationships and communications (Luft, and Ingham 1955). It is represented by a window divided into 4 quadrants - the top left window is called arena/open, the top right window is referred to as blind (spot). On the bottom left we have the façade/hidden window and the final window on the bottom right is the unknown quadrant (Verklan 2007). 
Johari Window

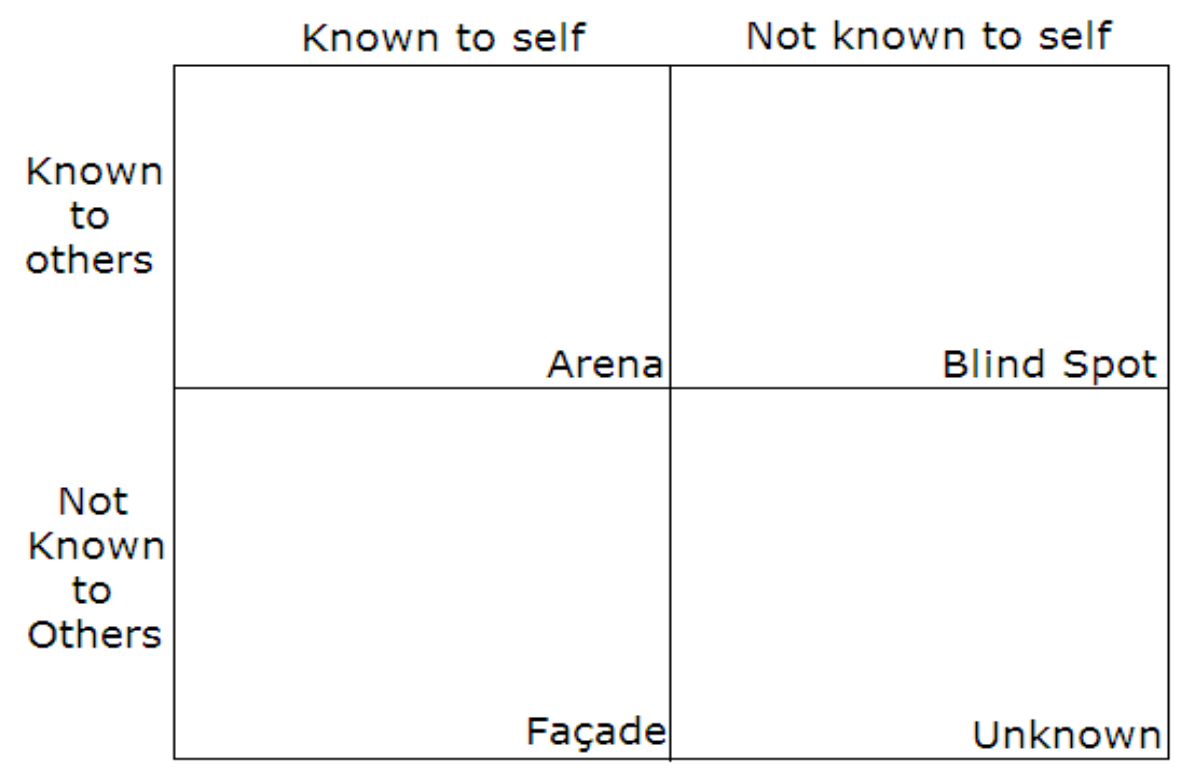

Figure 5: Johari Window

The arena quadrant represents characteristics that everyone, including the subject in question, knows. The façade quadrant consists of characteristics the subject knows about himself but keeps from others. The blind spot reveals characteristics that are unknown to the subject, but known to everyone else. Finally, the unknown contains characteristics that no one knows about the subject.

This model for interpersonal awareness has come a long way since the 50s and has since been applied to a vast array of learning situations and educational tools (Halpern 2009). My proposal is to adapt it for linguistic means. The original consists of two variables, number of persons and knowledge, thus my adapted model consists of two variables too. In this case, the variables will represent different language features.

\section{Results}

We are now ready to research data that will be analyzed with "four different pair of eyes», since each method comes from a different scientific field (tenet number one: interdisciplinary). These four different methods are my four pillars that serve as foundation for my PhD research. They are also my proposal to put a new spin on linguistic research on phonetic difficulties that so far has not improved the pronunciation of EFL learners all around the world (tenet number two: originality).

The 4 methods that ideally converge together to improve future research are:

1. Contrastive Analysis Hypothesis (Linguistics)

2. Statistics (Mathematics) 
3. Spectrographic Analysis (Physics)

4. Johari window (Psychology)

As usual, CAH and statistics, continue their rapport by giving statistical explanations to linguistic differences or similarities. So far so good. But now let us look at what the other two methods can contribute and let us start with the Johari window.

The original definition of what it does, has been, alongside with the Johari window itself, modified by me in order to adapt to my needs. As of now, this adapted Johari window is a technique that helps linguists better understand the relationship between speech characteristics.

For my PhD research on phonetical difficulties with English vowels, I focused on rhythm and vowel systems in order to produce original results. Using these two features as variables in comparing different languages against English helps us add a different point of view to the standard CAH method used in linguistics. English, as we know, is a stress-timed language with 5 vowels with 13 vowel phonemes in the RP variant.

The first feature, rhythm (also called isochrony), has been defined as language rhytmically dividing time into equal portions. Up until recently, linguists assumed there were three ways in which language can divide time:

1. Stress-timed, where the duration of every syllable is equal

2. Syllable-timed, where the interval between two stressed syllables is equal

3. Mora-timed, where the duration of every mora is equal

However, current research argues that the difference between syllable-timed and mora-timed is neglectable, and subsequently, there are only two rhythms (Nespor, Shukla, and Mehler 2010) . For my Johari window it is ultimately irrelevant since I am not using mora-timed language for my research, but in order to use the Johari window effectively I distinguished thus only between stress-timed and syllable-timed languages.

My second feature, vowel system, is a very uncommon way to differentiate languages, but since my research focuses exclusively on phonetic difficulties with vowels, it is only right to separate languages into two camps. On the one hand, we have languages that have 5 vowels and precisely 5 vowel phonemes. I defined these languages as having simple vowel systems. On the other hand, we have languages that also have 5 vowels, but more than 5 vowel phonemes, which means that every vowel has several different vowel phonemes. These languages have complex vowel systems.

With my distinctions clearly explaind the resulting adapted Johari window would look like this: 


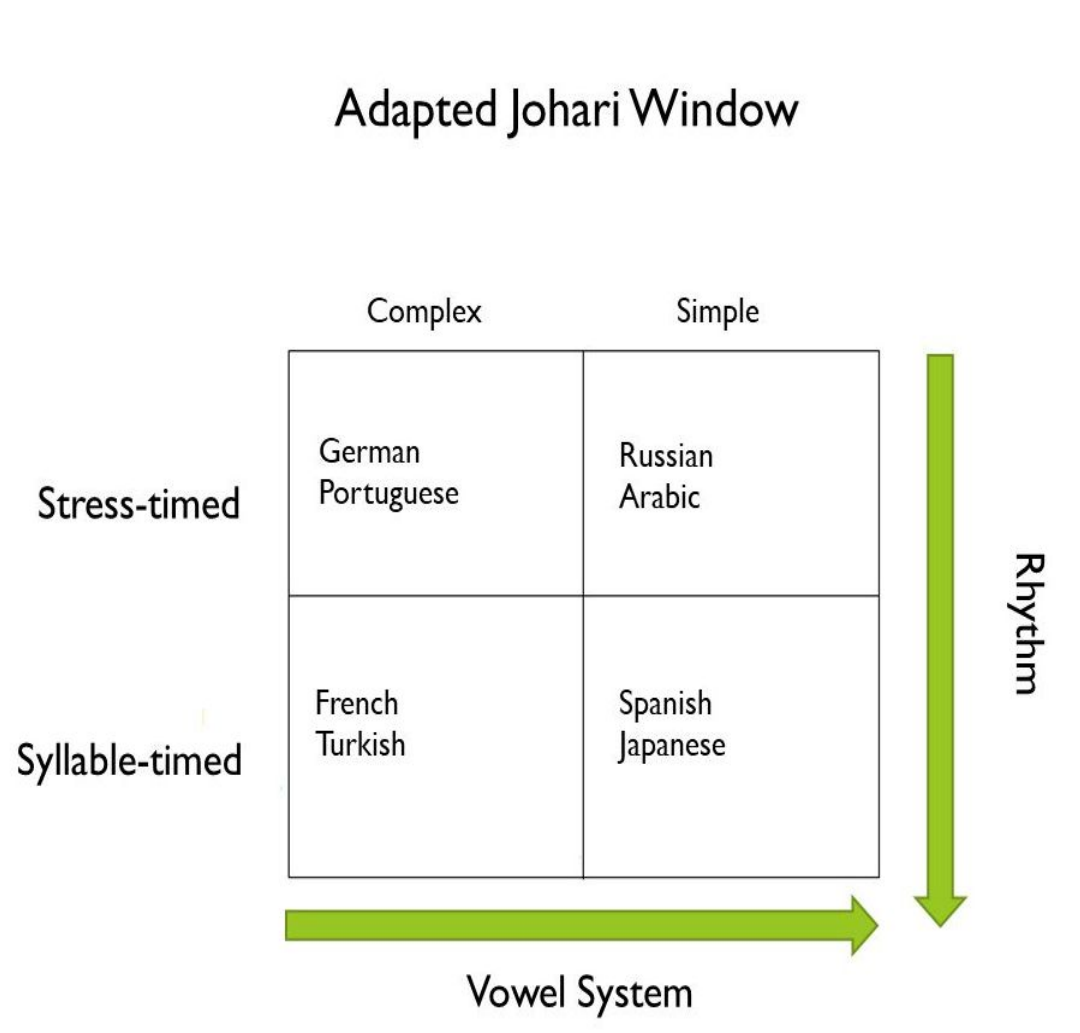

Adapted Johari Window

Figure 6: Adapted Johari Window

In the top left window, we have languages like German or Portuguese that share both the rhythm (stress-timed) and the complex vowel system with English. The top right window includes languages like Russian or Arabic, that share the rhythm (stress-timed) but not vowel system with English(both languages have a simple vowel system). Continuing with the bottom left window, we have languages like French or Turkish, that share the complex vowel system, but not the rhythm (both are syllable-timed) with English. Finally, the bottom right window, includes languages like Spanish or Japanese, that share neither rhythm, nor vowel system with English.

With the Johari Window fully defined, we come to the final part. Usually, when you record EFL learners from different languages, you analyze their speech afterwards with a native speaker of the language you are interested in. At best, you are able to find a helpful English professor with a background in linguistics that is willing to spare hours and hours to analyze several dozens of recorded data.

Let us just ignore the fact, that sometimes the native speaker you need to analyze the data, has no linguistic knowledge, or even an official teacher diploma. Let us instead be bothered by the fact, that even native speakers, sometimes, can have problems correctly reviewing English pronunciation. This could lead in some cases to skewed results, where the researcher was stricter than the native speaker, when it came to analyzing speech recordings.

This is where my final contribution comes in, the spectrographic analysis. Every phoneme occurs at certain frequencies, which means 
that modern software analyzes objectively if the spoken phoneme occurs at the right frequency. I will demonstrate this with a spectrogram of the word «turnout».
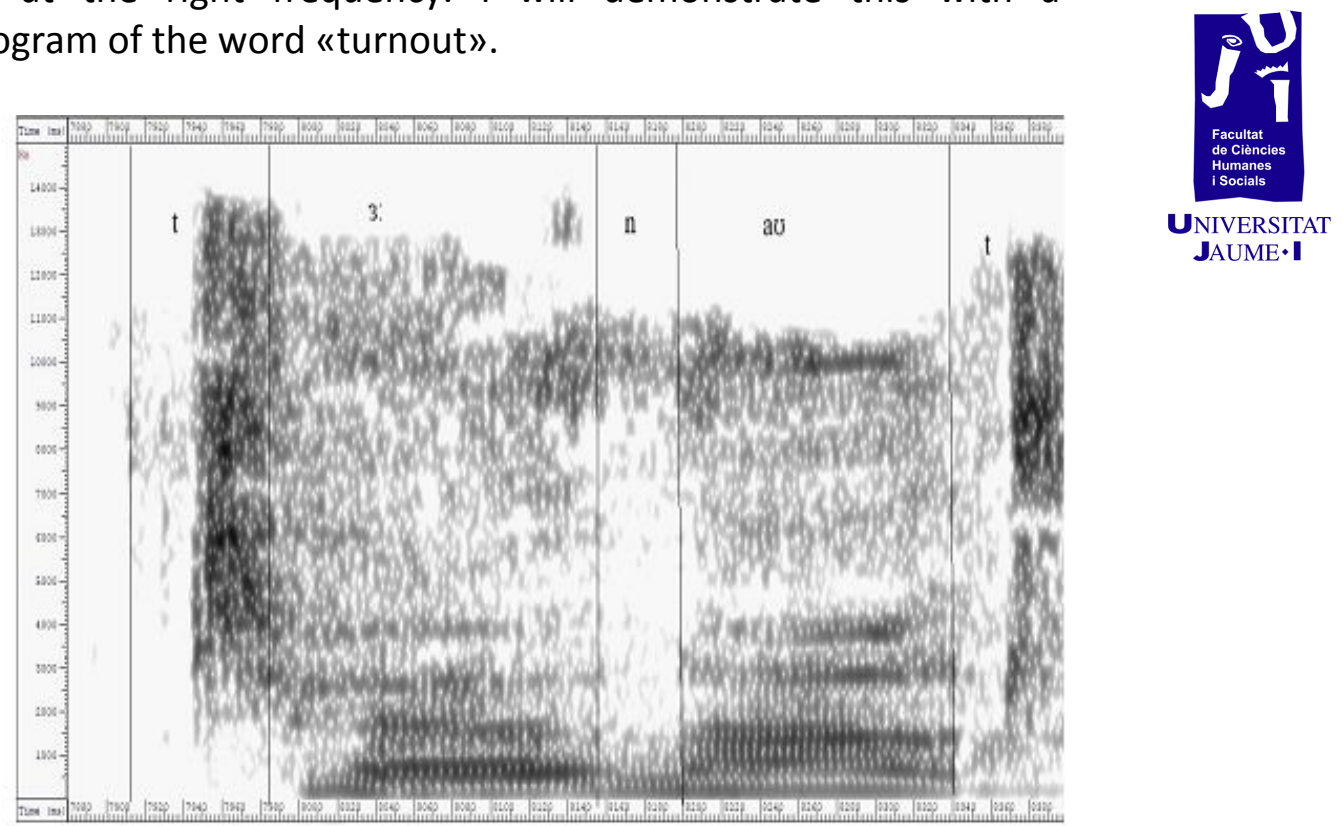

Figure 7: Spectrogram of «turnout»

Vowels are easily recognizable on a spectrogram because of formants. They are peaks of amplitude around a given frequency in the speech wave due to a resonance of the vocal tract. There are usually many formants for a periodic sound and each formant depends on the position of the tongue and lips. Graphically they are represented by clear bands of energy and their location and the distance between each of the formants tells us roughly the identity of the vowel. Since these formants occur at relatively lower frequencies ( $F 1$ occur from $250-900 \mathrm{~Hz}$, while F2 occur from 2700$800 \mathrm{~Hz}$ ) we'll zoom in on the spectrogram (Ball, and Rahilly 1999).

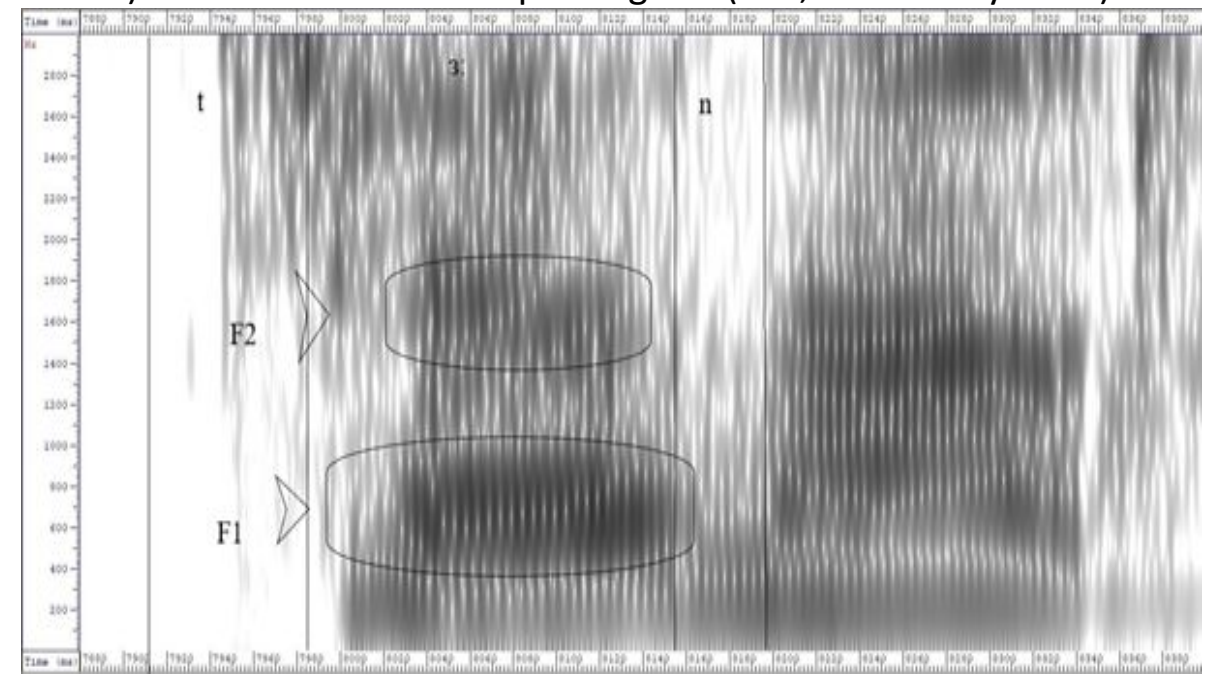

Figure 8: Zoomed in Spectrogram of «turnout» 
They occur on lower frequencies due to the fact that the first formant (henceforth F1) relates to the tongue height while the tongue location relates to the second formant (F2). You can clearly see the darker sections. These are the formants for /3:/. Finally, the amount of energy used for a prolonged period of time shows that it is the long vowel /3:/.

Every vowel phoneme has clearly defined formants, and thanks to modern software we can actually analyse if the phoneme is spoken correctly, thus adding an additional second opinion, besides the native speaker's analysis. Having two sets of results is extremely useful, not because of the similar results, but especially when the software and the native's ear classify a phoneme differently.

\section{Conclusions}

All these different methods from different fields, united to gain additional information on language learning, serve one small purpose: to serve as a starting point. My idea has always been to create data; data that could help others. Data that could inspire others, to take the baton and keep investigating in this direction. Or realizing it is actually the opposite direction. It does not matter, whether we gain knowledge one way or another.

All this research was never about lofty dreams of solving the conundrum of language learning, the Gordian Knot of phonetic difficulties. It was always about interdisciplinarity and originality. And (hopefully) delivering some good work. This may be some food for thought for upcoming researchers looking at the same problems linguists have for over a century, but hopefully seeing something different which may provide an actual solution to an old problem.

Let us recall the words of the Hungarian Nobel prize winner for biology, Albert Szent Gyorgyi: «be modest in everything, except your aims».

\section{References}

Ball, Martin, and Joan Rahily. 1999. Phonetics: The Science of Speech. London: Routledge.

Banathy, Bela, Edith Trager, and Carl Waddle, eds. 1966. "The Use of Contrastive Data in Foreign Language Course Development.»In Trends in Language Teaching, edited by Albert Valdman. New York: McGraw-Hill.

Cantos Gómez, Pascual. 2013. Statistical Methods in Language and Linguistic Research. Sheffield: Equinox. 
Halpern, Helen. 2009. "Supervision and the Johari Window: A

Framework for Asking Questions.» Education for Primary Care 20 (1): 10-14.

König, Ekkehard, and Volker Gast. 2009. Understanding EnglishGerman Contrasts. Berlin: Erich Schmidt Verlag.

Ladefoged, Peter, and Keith Johnson. 2011. A Course in Phonetics. Belmont: Wadsworth.

Luft, Joseph, and Harry Ingham. 1961. "The Johari Window, a Graphic Model of Interpersonal Awareness.» Human Relations Training News 5 (9): 6-7.

Michalewicz, Zbigniew, and David Fogel. 2000. How to Solve It: Modern Heuristics. Berlin: Springer Verlag.

Nespor, Marina, Mohinish Shukla, and Jacques Mehler, eds. 2011. "48 Stress-timed vs. Syllable-timed Languages.» In The Blackwell Companion to Phonology, edited by Marc van Oostendorp, Colin J. Marc, Elizabeth V. Hume, and Keren Rice, 48. Hoboken: Wiley.

Newmark, Leonard, and David Reibel. 1968. "Necessity and Sufficiency in Language Learning." International Review of Applied Linguistics in Language Teaching 9 (1-4): 145-164.

Oller, John, and Said Ziahosseiny. 1970. "The Contrastive Analysis Hypothesis and Spelling Errors.» Language Learning 20 (2): 183189.

Saito, Hiroko, and Michael Ashby. 2013. «Edward Gauntlett's Phonetics (1905).» Paper presented at the Proceedings of the Phonetics Teaching and Learning Conference 2013.

Stockwell, Robert, and Donald Bowen. 1967. «The Sounds of English and Spanish.» Chicago: The University of Chicago Press.

Verklan, Terese. 2007. «Johari Window: A Model for Communicating to Each Other." The Journal of Perinatal \& Neonatal Nursing 21 (2): 173-174. 



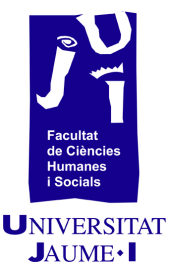

\section{Humanitats, Interculturalitat, Història i Patrimoni}

Humanidades, Interculturalidad, Historia y Patrimonio Humanities, Interculturality, History and Heritage 



\section{De la ciutadania patriòtica a l'exclosa:}

dones decents i prostitutes sota el primer franquisme

Javier Lara Fonfría

al259489@uji.es 
I. Resum

L'1 d'abril de 1939, es posava fi a la Guerra d'Espanya i s'hi va instaurar un Estat totalitari que va portar a terme una repressió política i moral que va dividir el país entre vencedors i vençuts. Així doncs, l'objectiu principal d'aquest treball és analitzar i interpretar com d'aquest derivà un monisme moral, els fonaments ideològics del qual foren el feixisme, amb la Falange i la Sección Femenina, i l'Església, amb encara més preponderància que el feixisme. Així mateix, volem estudiar com es configurà el model que havia de ser la bona mare i esposa: característiques de la perfecta espanyola. La hipòtesi principal és, doncs, que la dona preferida pel règim era aquella que estava amerada de moral nacionalcatolicista.

Pel que fa a la metodologia, ha estat cabdal la revisió bibliogràfica de les obres que es detallaran dejús. D'altra banda, s'han emprat fonts primàries, principalment el Butlletí Oficial de l'Estat, per la qual cosa les lleis i els decrets publicats en aquest han servit per a demostrar com la moral es va jurisdiccionalitzar i que la Iluita contra la prostitució va suposar, en part, una forta càrrega moralitzant i culpabilitzadora d'aquesta. Això no obstant, aquestes fonts primàries no s'han inclós en el present article, únicament en el TFG del qual és originari.

Paraules clau: franquisme, construcció del gènere, dona, Sección Femenina, matrimoni maternitat, Patronato de Protección a la Mujer, prostitució.

\section{Introducció}

Acabada la Guerra d'Espanya, el país necessitava urgentment reconstruir-se, però el règim entenia que aquesta reconstrucció tenia una clara faceta moral que resultaria de vital importància. Així doncs, aquest serà l'objecte d'estudi del treball present: el procés mitjançant el qual el franquisme estableix un monisme moral que, en qüestió del gènere, es tradueix en la definició de la feminitat amb el matrimoni i la maternitat.

Ara bé, tot i la pretensió d'hegemonia del model de dona que el règim volia fer prevaldre, aquest tenia escletxes, ja que no totes les dones foren sagramentades amb el matrimoni ni tampoc totes les dones foren mares dins del matrimoni. A més a més, hi ha un altre aspecte que aporta novetat a aquest estudi i és la hipòtesi que, tot $\mathrm{i}$ que el model de dona no fora reproduït de forma hegemònica, el franquisme delimità també en quina manera el rol de gènere es podia trencar i en quines no. És a dir, la ruptura de les dones amb el 
que s'esperava d'elles sols es reconeixia des de la prostitució, però no des del lesbianisme.

\section{Objectius}

Així les coses, l'objecte d'estudi d'aquest treball és el procés mitjançant el qual el franquisme, amb la instauració d'un monisme moral, profundament cristià i catòlic, construí el rol de gènere femení.

Principalment, es pretén analitzar i interpretar de manera crítica com les dones experimentaren una forta repressió que, durant el primer franquisme (1939-1959) -que tot i l'abast extens de l'aparell repressor del Nuevo Estado requeia sobre el conjunt total de la població-, ho feu d'una manera especial i més marcada, sobre les dones, únicament per raó del seu gènere.

A més, a continuació esmentem els objectius secundaris. El primer és analitzar el context de repressió i la política de gènere desplegada pel règim, de la qual foren protagonistes les dones, ja que tot i no ser mai considerades subjecte polític, ${ }^{1}$ sí que se'n van considerar objecte, i el paper del nacionalcatolicisme en aquest procés.

Conseqüentment, un dels altres objectius secundaris del treball és estudiar quin tipus de dona s'espera des del règim de Franco, el qual reduïa la feminitat a ser esposa i mare. Tanmateix, també es tindran en compte quins models s'empren de forma propagandística, per tal que la política de gènere, de la qual fins i tot podria dir-se que era més una doctrina, arribe d'una manera més fàcil a la població.

En tercer lloc, cal assenyalar que aquests models no foren realment hegemònics, sinó que hi existiren clares ruptures, de les quals la que més ressò tingué va ser la prostitució. D’aquesta manera, també serà objecte d'estudi amb quines institucions i quins mecanismes es criminalitzaren aquests contramodels.

Finalment, sols queda destacar un objectiu i aquest és l'estudi de la sistematització de l'aparell repressor que, entre d'altres, participarà en la construcció del gènere femení, criminalitzant tot allò que apartara de la dona de l'ideal de mare i esposa. És a dir, es pretén demostrar que aquesta repressió, amb un clar rerefons moral, estigué institucionalitzada i burocratitzada, allunyada de qualsevol matís de descontrol. 
El present treball presenta les característiques d'un estudi elaborat a partir d'una revisió bibliogràfica de la qual s'obté el marc teòric necessari per al seu desenvolupament, alhora que s'han consultat fonts primàries per tal de recolzar el que es diu respecte a la jurisdiccionalització i burocratització de la moral.

Cal ressenyar que aquest estudi és fruit del Treball de Final de Grau, del grau en Humanitats: Estudis Interculturals de la Universitat Jaume I, de la mateixa autoria que aquest article, i que fou defensat en octubre de 2018. Comprén el període des de final de la guerra, el primer d'abril de 1939, fins a l'any 1959, el que històricament es coneix com a primer franquisme. Pel que fa a la data inicial, es pren com a referència el final de la guerra, ja que el que interessa és l'estudi i la interpretació de com el Nuevo Estado engega tot l'aparell repressor ideològic, la qual cosa es va produir amb la fi de la contesa. Tanmateix, s'ha de tindre en compte que ja durant la guerra, i fins i tot abans d'aquesta, es configurà la moral adoptada pel règim, per la qual cosa l'estudi és conjuntural, ja que no pot reduir-se a la delimitació de l'1 d'abril de 1939.

Per altra banda, hem assenyalat el 1959 com a punt final del moment que ens ocupa. Això és perquè es considera que, tot $\mathrm{i}$ tractar-se d'una divisió amb finalitat didàctica, no deixa de ser útil; la situació de la dona, de manera generalitzada, no serà la mateixa des d'aleshores. Aquest canvi es deu, d'una part, a la prohibició de la prostitució i dels centres de tolerància, promulgada per decret llei el 3 de març de 1956, i de l'altra, a l'entrada d'Espanya en I'ONU el 1955; i és que aquestes dues fites històriques marcaren l'inici d'un aperturisme que, si bé no allunyà el règim de la seua ideologia, sí que ho feu de la rigidesa dels primers anys. A més a més, cal no oblidar que aquesta divisió i l'inici de l'anomenat aperturisme guanyà més sentit si es té en compte la fi de l'autarquia.

Pel que fa a les fonts secundàries necessàries per a la revisió bibliogràfica, hem de destacar algunes que hem emprat més i més a fons. Entre d'altres, hi ha, pel que fa al context històric de l'Espanya del moment, La represión como sistema (2012), de Julio Aróstegui, i El factor católico en la política española. Del nacionalcatolicismo al laicismo (2006), de Rafael Díaz Salazar. Quant a la socialització del gènere $i$ als models que s'adopten per al femení, les obres més destacades són La mujer y la educación física durante el franquismo (2008), de Juan Manrique Arribas; Women and Spanish Fascism: The Women's Section of Falange, 1934-1959 (2004), de Kathleen Richmond, i El desfile de lo femenino: las mujeres de la Sección Femenina en el calendario y las celebraciones franquistas (2017), de Francisco Jiménez Aguilar. Finalment, pel que fa a la contextualització de la prostitució i els diferents aspectes tractats en 
el treball relatius a aquesta, ha estat Assumpta Roura l'autora més emprada, amb obres com ara Mujer y moralidad durante el primer franquismo (2005) i Mujeres para después de una guerra. Una moral hipócrita del franquismo (2003). Així mateix, hem recorregut a altres obres com ara Prostitutas legales y clandestinas en el franquismo (2003), de Mirta Núñez, i La prostitución en la España contemporánea (2003), de Jean-Louis Guereña.

En relació amb les fonts emprades per a demostrar la burocratització i jurisdiccionalització de la moral, hem utilitzat fragments obtinguts del Butlletí Oficial de l'Estat (BOE), mitjançant les bases de dades següents: Legislación histórica de España (18101978) del Parlament Espanyol, Gazeta: Colección Histórica (BOE), i la Biblioteca Nacional de España.

\section{Breu contextualització: dels objectius militars a la croada espiritual}

Amb el darrer comunicat oficial de guerra, es posava fi a la contesa bèl-lica. Les tropes revoltades havien assolit els darrers objectius militars, començava ara la pugna i la porga per la moral. El Nuevo Estado engegaria tot un aparell repressor que, lluny d'estar desorganitzat i respondre a acciones espontànies d'individus afectes a la seua ideologia, fou la resposta d'un plantejament estratègic dels conspiradors. I és que al cap i a la fi no podem oblidar que «a la Guerra Civil española le siguió una larga paz incivil. [...] Franco [...] estableció un estado represivo sobre las cenizas de esa guerra, persiguió sin respiro a sus oponentes y administró un creuel y amargo castigo» (Casanova i Gil, 2009: 231).

Així, la destrucció dels vençuts es convertí en prioritat absoluta, després de la qual va començar un període d'execucions massives, d'empresonaments i de tortures per a milers d'homes i dones. Aquest aparell repressor s'inicià el 9 de febrer de 1939, amb la Llei de Responsabilitats Polítiques, la qual tenia caràcter retroactiu fins al primer d'octubre de 1934 (Aróstegui, 2012: 93-94). El que es pretenia era la mort civil, a banda de biològica, dels oponents, ja que la depuració política estava adreçada a assolir una nova moralitat, una nova espiritualitat; en definitiva, una ètica que fonamentara el nou Estat. Hi va haver diferents lleis que acabaren establint un monisme moral que configurà l'Espanya de la postguerra, la més destacable de les quals és la que oficialment es denominà Causa General, amb què es pretenia la recaptació de tota la informació possible sobre les actuacions considerades contràries al Movimiento des del 18 de juliol de 1936.

Esa maquinaria de terror organizado desde arriba, basada en la jurisdicción militar, en juicios y consejos de guerra sin las mínimas garantías, requería una amplia participación "popular", de 
informantes, denunciantes, delatores, entre los que no sólo se encontraban los beneficiarios naturales del la victoria, la Iglesia, la Falange y la derecha de siempre. La purga era, por supuesto, tanto social como política y los poderes de la comunidad, la gente de orden, las autoridades, aprovecharon la oportunidad para deshacerse de los "indeseables", "animales" y revoltosos. [...] La barrera entre los vencidos y los vencedores podía cruzarse a través de la delación, de esa denuncia que le libraba a uno de las sospechas. [...] El conformismo religioso era recomendable para los que querían un trabajo, mejorar su posición o cualquier otro ascenso o seguridad.

Amb l'Església catòlica com a pilar del règim, els revoltats tenien el vistiplau i la benedicció de la Santa Seu. Així doncs, els components fonamentals que alimentaren la ideologia del franquisme foren el resultat d'elements conservadors i ultraconservadors que s'aferraven a la tradició, encarnada per Franco, hereu de l'Espanya anacrònica i temorosa del progrés (Aróstegui, 2012: 123-124).

En aquest context, la repressió fou social i ideològica, però també fou moral; i, basant-se en aquesta, es justificaria la persecució social i ideològica dels vençuts. Espanya no sols era «Una, Grande y Libre», sinó que era "Una, Grande, Libre» i sobretot catòlica. De fet, la unió de pàtria i religió -que és inqüestionable-donaria lloc a una ideologia característica: el nacionalcatolicisme. Aquesta fou l'aglutinant dels diferents grups que conformaren el bàndol revoltat, unificadora alhora que hegemònica. En altres paraules, «l'espasa i la creu, [...] les autoritats civils, les militars i l'Església anaven de bracet» (Gavaldà, 1994: 30-31). Així, el nacionalcatolicisme, com a fonamentalisme totalitari, impregnà per complet la societat espanyola.

Així les coses, amb el nacionalcatolicisme com a horitzó ideològic, el prototip de bona espanyola s'implantà, o almenys així s'intentà, amb rigor, de manera que alguns col-lectius havien de quedar fora de la construcció del rol de gènere femení que s'entenia com a moralment perfecte, a més d'exigible. Aquests col-lectius foren prostitutes i lesbianes, ja que:

La iglesia impuso una ética social y sexual basada en el matrimonio indisoluble, monogámico y sexualmente vergonzante. La Dictadura y la Iglesia convirtieron deliberadamente la familia en la columna vertebral del sistema y en un instrumento eficaz de control de la sexualidad (Galván, 2010: 136).

L'Espanya tradicional i catòlica instaurà uns clars rols de gènere que marcaran no sols el paper de la dona dins del matrimoni, sinó que també adjudicarà uns models ben delimitats, i del tot imprecisos, per a aquelles que no segueixen l'ortodòxia catòlica, tot 
tenint en compte que, sovint, aquests estereotips no concorden de forma absoluta amb la realitat. A més a mes, cal assenyalar que la repressió moral fou selectiva, és a dir, dirigida de manera deliberada contra la dona, i és que la seua castedat era considerada de més difícil verificació.

La sensibilitat femenina quedà atrofiada, se li ha donat una formació moral que la fa creure que el sexe és brut i cal menysprearlo, de manera que preservar la virginitat era un dogma. D'altra banda, però, els homes, educats i socialitzats diferents, en els anys de més rigidesa de la moral sexual catòlica, s'iniciaren en la sexualitat de la mà de prostitutes, amb el vistiplau de l'ampla màniga d'un règim patriarcal com el del franquisme.

\section{Dones per a després d'una guerra}

Una nova dona per a una nova era, per a una nova Espanya. Aquesta fou la línia d'actuació del règim, per a unes dones que havien de ser el sagrari de la raça hispànica, que havien de consagrar en la unitat de la fe i de la nació, els nous espanyols a què donarien llum. I és que al cap i a la fi "La guerra es de los hombres; la posguerra para las mujeres. A ellas les corresponde ahora enderezar la cotidianidad como si nada hubiese ocurido» (Roura, 1998: 239).

D'aquesta manera, a l'Espanya de Franco era necessària una dona regenerada de la qual tornaria a nàixer la Nació, igual que a la Itàlia de Mussolini, ja que

Mussolini's regime stood for returning women to home and hearth, restoring patriarchal authority, and confining female destiny to bearing babies (De Grazia, 1992: 1-2).

La necessitat d'una nova dona fou un aspecte ideològic, propi del feixisme espanyol així com dels feixismes italià i alemany, però també tingué un caràcter moral i que, per tant, també es va legislar, es va jurisdiccionalitzar, de manera que quedà la dona en un rol social d'eterna tutela, l'honor i castedat de la qual ha de salvaguardar el govern de la nació.

Moltes d'aquestes dones hagueren d'experimentar una doble

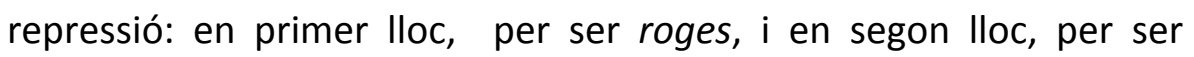
dones, la qual cosa es tradueix en que l'adulteri es considerava de manera diferent per a homes i per a dones, ja que sobre aquestes la llei requeia d'una forma més contundent que sobre els homes; i no oblidem que la condemna de l'adulteri no era pel delicte per se, sinó pel greuge sofert pel marit, ja que fins i tot aquest podia anul.lar la condemna si ho considerava oportú.

D'aquesta manera, el model de dona que es pretén implantar és el de la dona domèstica, o podríem dir més bé domesticada, ja que la 
dona espanyola, com els opositors al règim, havia trobat amb la fi de la guerra la mort civil. La seua identitat havia de construir-se del brancal cap a dins, ja que de brancal cap a fora sols podia estar reconeguda com a esposa, mare, filla o germana.

Així les coses, la Sección Femenina serà la responsable de l'enquadrament $^{2}$ de la dona en el Nuevo Régimen. D'aquesta manera, es produeix una categorització d'allò que han de representar cadascun dels sexes, categorització que assigna a homes i dones unes funcions determinades en la societat, però que les imposa el sexe masculí. D'aquesta manera, l'únic espai de poder que tenia la dona era el domèstic, però tampoc plenament, ja que l'autoritat del marit continuava sent inqüestionable.

Ara bé, la dona, a causa del seu confinament a la vida privada, tenia un paper que era fonamental si el règim, com qualsevol altre, volia perpetuar-se, ja que fou l'encarregada de transmetre models i valors ideològics, religiosos i cívics (Manrique, 2008: 75). I és que tot $\mathrm{i}$ tractar-se d'una societat patriarcal, podem veure que la dona era cabdal per al règim, d'igual manera que ho fou per a altres règims totalitaris, com foren els de Hitler a Alemanya i el de Mussolini a Itàlia. Pot resultar controvertit aquest paper protagonista de la dona, i encara més en un país com Espanya, però de fet

Koonz's book [...] showed that contrary to received opinion, women had played significant roles in the movement and regime. Indeed, women had used the Nazi's idealisation of the family to empower themselves, and had been primarily responsible for the social component of Nazi policies. (Passmore, 2003: 1).

Amb tot, el que no ha de resultar estrany és que, amb aquest organisme paral·lel a Falange, la dona fora, en certa mesura, partícip de la vida pública i és que «la visión que tenía la SF de la mujer como participante activa en la reconstrucción económica y espiritual de la nación armonizaba con el ideal fascista de una población movilizada» (Richmond, 2004: 33). Pot veure's com la Sección Femenina promou, fins a cert punt, el treball de les dones, així com l'educació d'aquestes, no sols primària, sinó fins i tot secundària i superior, però sempre respectant, com a normes bàsiques, el fet de no competir amb els homes, evitar la frivolitat i la pedanteria. És a dir, el fet que les dones no foren subjecte polític no significa que el règim no desplegara una política de gènere - de la qual la dona fou objecteque es va fer realitat a través dels programes educatius de la Sección Femenina (Richmond, 2004: 35-46).

Però aquest reconeixement de les dones com a protagonistes de la política, en cert sentit, estava enverinat, ja que es tractà d'un fals

2 Expressió emprada i extreta de l’obra Encuadramiento femenino, socialización y cultura en el franquismo, de la qual és editora Lucía Prieto Borrego. 
apoderament. En primer lloc, s'ha de ressenyar que les professions principals encapçalades per les dones en l'esfera pública havien estat feminitzades, com ara el magisteri i la infermeria, i en segon lloc és interessant destacar que aquest reconeixement fou possible gràcies al fet que en l'ideari joseantonià, que bevia del feixista, les dones havien de ser «gestoras eficaces de los espacios privados gracias a los cursos de economía doméstica que se impartían en las escuelas para niñas» (Aguado i Ortega, 2011: 239).

Com ja s'ha vist, la política que el franquisme va imposar sobre la dona reduïa la seua existència a la maternitat, que sempre havia d'estar emmarcada dins del matrimoni. Ara bé, com afirma Jiménez Aguilar, quan parlem de les dones durant el franquisme no podem reduir la seua posició política a ser únicament l'àngel de la llar, sinó que les dones van tindre un paper important en l'agenda política del règim, ja que també en participaren. ${ }^{3}$ Tanmateix, també s'ha de ressenyar que el matrimoni i la família que havia de formar eren un deure ineludible per a ser patriòtic, catòlic i bon espanyol, de manera que l'Estat es va convertir en el custodi de la unitat familiar. I és que, al cap i a la fi, el matrimoni era un sagrament, i l'Espanya nacionalcatòlica no podia desprotegir el fruit més important d'aquest: els fills.

Així, la presència de les dones en els actes i les festivitats del règim va esdevindre una pràctica bastant habitual. Per al règim n'era necessària la legitimació, i aquesta es va portar a terme, entre altres maneres, amb tota una simbologia que es veuria plasmada en les celebracions polítiques, religioses o populars, fins a configurar el que alguns autors ${ }^{4}$ han anomenat "la cultura de la victòria» ${ }^{5}$.

Es va institucionalitzar una sèrie de festivitats, aprovades per ordre ministerial el 9 de març de 1940 i publicades en el BOE, i en aquestes les dones comptaren com a contingent social indispensable, ja que la participació femenina en les litúrgies i els rituals diferents prengué un caràcter simbòlic. A més a més aquests símbols valgueren per a transmetre models de conducta per mitjà de

3. És clar que creure que la participació de la dona en la vida pública durant el franquisme fou signe d'emancipació és del tot ingenu, ara bé, el que no pot negar-se és que, d'una manera o altra, la dona va resultar apoderada, encara que aquest apoderament haguera estat parcial i responguera a les directrius del feixisme i fora de marcat caràcter patriarcal. Així, s'ha d'entendre com a reconeixement de la importància de l'àmbit reproductiu, en tant que part essencial del sistema econòmic, no en el sentit actual que el feminisme del segle XXI empra el terme apoderament.

4. Com ara Miguel Ángel Del Arco Blanco, Claudio Hernándes Burgos, o Teresa María Ortega López.

5. Pot entendre's com «cultura de la victòria» el discurs hegemònic impulsat pels revoltats, fins i tot ja durant la guerra, que pretenia consolidar la seua base social, i la del futur nou règim mitjançant la promoció i l'exaltació de la victòria del bàndol insurgent, la qual es tenia per justa, ja que la guerra no es va veure únicament com una guerra civil, sino especialment com una croada religiosa contra aquells que representaven l'antiEspanya. Aquest discurs no fou l'únic recurs legitimador del Nou Estat, però sí el més important, ja que va afavorir la identificació i la rememoració de l'enemic comú alhora que l'experiència bèl-lica compartida permetia reforçar la cohesió interna dels heterogenis suports socials de la dictadura (Muñoz, 2017: 31). 
festivitats com ara las hijas de Teresa, las plañideras del ausente i las labradoras de San Isidro. Tanmateix, la simbologia no es va veure reduïda a la pompa i a l'ostentació, sinó que va agafar forma amb models més fàctics com ara la Mare de Déu, Santa Teresa de Jesús, Isabel I de Castella o Carmen Polo de Franco. Amb aquests models, aconseguia transmetre els preceptes morals més estrictes, això no obstant, la prostitució comptà amb el vistiplau del règim fins 1956. Heus ací, de nou, la doble moral (Ñúñez, 2003: 18-22).

El 25 de març de 1942 es constitueix el Patronato de Protección a la Mujer, hereu d'El Patronato Real para la Represión de la Trata de Blancas. Així doncs, es reprenia la tasca de «la dignificación moral de la mujer, especialmente de las jóvenes, para impedir su explotación, apartarlas del vicio y educarlas con arreglo a las enseñanzas de la religión católica» (Roura, 1998: 48).

Amb tot, cal preguntar-se fins a quin punt l'organisme recentment constituït pretenia protegir les dones de la tracta de blanques i no criminalitzar-les. És il-lustratiu el fet que en el mateix acte en què el Patronato de Protección a la Mujer s'establí, s'acordà que havia de tindre un sentit profundament cristià, ja que l'organisme s'havia d'atendre «al celestial patronato del Buen Pastor, de Nuestra Señora de los Dolores y de Santa Micaela del Santísimo Sacramento" (Roura, 1998: 48). Aquesta moral ben bé pot anomenar-se moralitat, ja que, al cap i a la fi, el que hi ha al rerefons de tot no és únicament la preocupació moral, sinó la culpabilització i la criminalització de les prostitutes -que no de la prostitució-, és a dir, el pes d'aquesta moral hipòcrita recau sobre elles i no sobre les necessitats que portaren a moltes a la pràctica de la prostitució.

\section{Conclusions}

Abans que res, cal afirmar que els objectius declarats en la introducció s'han assolit i, per tant, la conclusió principal a què s'ha arribat és que en el clima generalitzat de repressió que caracteritzà la postguerra espanyola, les dones experimentaren una doble opressió, pel simple fet de ser dones.

A més, es pot concloure que l'opressió que la societat espanyola va haver d'experimentar es degué, en gran part, a la participació de l'Església catòlica en l'aparell repressor, i a l'estreta unió entre Església i Estat, el conegut com nacionalcatolicisme. D'altra banda, es pot resoldre que el model de dona, així com el procés de socialització d'aquesta, estigueren clarament delimitats per l'aliança entre Església i feixisme, per la qual cosa, el rol que la dona havia d'exercir era el de la bona mare i esposa, sempre sota el mantell del sagramentat matrimoni, l'única via que hi havia per conformar una vida honrosa i acceptable. 
A més a més, aquest treball permet deduir que sovint es produïen fractures que feren que la dona, com a temple de la raça, haguera de tindre en compte els perills que podien assetjar-la. El més destacat, i que més immoralitat podia causar, era el de la prostitució. N'hi havia d'altres menys greus, però igualment deshonrosos com ara la maternitat fora del matrimoni o l'avortament. Ara bé, com a conclusió principal pel que fa a la prostitució, cal assenyalar que la immoralitat en aquest cas esdevé, més que per la prostitució per se, perquè la dona s'haja prostituït, perquè això comporta la pèrdua de I'honradesa i l'aparta de la seua finalitat: el matrimoni i la maternitat.

Finalment, sols queda assenyalar que la repressió que portà a terme el franquisme no té res d'incontrolada, sinó que respon a tot un aparell burocràtic que el que va fer fou jurisdiccionalitzar la moral catòlica.

Amb tot, el que queda ben clar és que a l'Espanya de Quintero, León i Quiroga només hi havia una única manera que la dona es considerara decent, però això comportava tot un seguit de condicions que feren que l'espanyola d'aquests anys quedara totalment subjugada, més enllà del vessant polític, dominada per I'home en tots els espectres que la seua vida manifestara, pel que fa al públic, o productiu, perquè sols podia ser considerada com a objecte polític, i en el privat perquè se li atorgà, com s'ha vist, un apoderament fals i enverinat, que no va consistir en més que reconéixer, per influència del feixisme, la importància de l'àmbit reproductiu al nivell del productiu, però categoritzant-lo com a intrínsecament femení, ja que aquest fals apoderament va fer que els àmbits productiu i reproductiu foren encara més estancs.

Així doncs, el franquisme va haver de desenvolupar tot un aparell simbòlic amb què la dona poguera veure reflectit quin havia de ser el seu comportament, de manera que reforçava la construcció del gènere que es pretenia fer valdre, la qual cosa s'acompanyà de la legislació adient per tal de castigar, més que per conscienciar, les possibles immoralitats impermissibles en un Estat nacionalcatòlic.

\section{Bibliografia}

Aguado, Ana i Teresa María Ortega, ed. 2001. Feminismos y antifeminismos: culturas políticas e identidades de género en la España del siglo xx. València i Granada: Universitat de València i Universitat de Granada.

Aróstegui, Julio, coord. 2012. Franco: la represión como sistema. Flor del Viento: Barcelona.

Casanova, Julián i Carlos Gil Andrés. 2009. Historia de España en el siglo Xx. Ariel: Barcelona 
De Grazia, Victoria. 1992. How Fascim Ruled Women. Italy, 19221945. Berkeley i Los Angeles: University of California Press.

Díaz-Salazar, Rafael. 2006. El factor católico en la política española. Del nacionalcatolicismo al laicismo. Madrid: PPC Ediciones.

Gavaldà Torrents, Antoni. 1994. Quaranta anys sense llibertats. El Franquisme. Barcelona: Graó.

Guereña, Jean-Louis. 2003. La prostitución en la España contemporanea. Madrid: Marcial Pons.

Koonz, Claudia. 1987. Mothers in the Fatherland. Women, the Family and Nazi Politics. Nova York: St. Martin's Press.

Jiménez Aguilar, Francisco. 2017. «El desfile de lo femenino: las mujeres de la Sección Femenina en el calendario y las celebraciones franquistes». Conferència presentada en el VI Encuentro Internacional Jóvenes Historiadores en Historia Contemporánea. Accés del 23 de gener de 2018. https://historiazgz2017.files.wordpress.com/2017/05/m-4jimenez-francisco.pdf.

Manrique Arribas, Juan Carlos. 2008. La mujer y la educación física durante el franquismo. Valladolid: Universidad de Valladolid.

Núñez, Mirta. 2003. Mujeres caídas. Prostitutas legales y clandestinas en el franquismo. Madrid: Oberon.

Passmore, Kevin. 2003. Women, gender and fascism in Europe, 191945. Manchester: Manchester University Press.

Pérez Moreno, Heliodoro Manuel. 2010. «Paradojas de la Sección Femenina: Disonancias entre "Modelos de mujer" e instituciones formativo-asistenciales». En Encuadramiento femenino, socialización y cultura en el Franquismo, editat per Lucía Prieto Borrego, 177-196. Málaga: Centro de Ediciones de la Diputación de Málaga.

Prieto Borrego, Lucía, ed. 2010. Encuadramiento femenino, socialización y cultura en el Franquismo. Málaga: Centro de Ediciones de la Diputación de Málaga.

Richards Michael. 1999. Un tiempo de silencio. La guerra civil y la cultura de la represión en la España de Franco, 1936-1945. Barcelona: Crítica.

Richmond, Kathleen. 2004. Las mujeres en el fascismo español. La sección femenina de la falange, 1934-1959. Madrid: Alianza.

Rioyo, Javier. 2003. La vida golfa. Historia de las casas de lenocinio, holganza y malvivir. Madrid: Santillana Ediciones Generales. 
Roura, Assumpta. 1998. Mujeres para después de una guerra. Informes sobre moralidad y prostitución en la posguerra española. Barcelona: Flor del Viento.

Roura, Assumpta. 2005. Un inmenso prostíbulo. Mujer y moralidad durante el franquismo. Barcelona: Base.

Sanz Gavillon, Anne-Claire 2017. «Discursos de género y violencia política sexuada en las dictaduras de Franco y Pinochet. Paralelos, transferencias e influencia en la formación del pensamiento y la memoria feminista sobre la violencia de genero». Conferència presentada en el VI Encuentro Internacional Jóvenes Historiadores en Historia Contemporánea. Accés del 25 d'abril de 2018. https://historiazgz2017.files.wordpress.com/2017/05/m-4-sanzanne-claire.pdf.

Sopeña Monsalve, Andrés. 1996. La Morena de la Copla. Barcelona: Crítica.

Torres, Rafael. 2002. El amor en tiempos de Franco. Madrid: Oberon.

Vincent, Mary. 2003. Spain. The Primo de Rivera dictatorship. En Women, gender and fascism in Europe, 1919-45, editat per Kevin Passmore, 189-213. Manchester: Manchester University Press.

Werrie, Paul. 1966. El amor a la española. Barcelona: Sagitario. 



\title{
Comunicación para la paz:
}

\author{
análisis de las campañas \\ sobre personas refugiadas (UNICEF)
}

Neva Rubio Pérez

nevarubio@hotmail.com 
I. Resumen

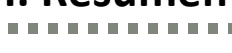

Actualmente la comunicación que llevan a cabo las ONGD tiene mucho peso en las temáticas sociales y humanas. UNICEF, al ser una de las principales organizaciones que trabaja por los derechos de la infancia, cumple un papel social importante en la transmisión de información y en la percepción de ciertas situaciones, en este caso, la de aquellas personas que buscan refugio. Este artículo trata de analizar qué tipo de comunicación, en términos de eficacia cultural, está haciendo UNICEF con respecto a quienes buscan refugio. Se trata de un análisis de dos campañas que realizó esta ONGD durante los años 2016 y 2017.

Palabras clave: personas que buscan refugio, refugiados, comunicación, ONGD, transformación social, advocacy, eficacia cultural.

\section{Introducción}

Este artículo de investigación es un análisis comparativo de las campañas de comunicación creadas por UNICEF para abordar la situación y dificultades por las que atraviesan las personas que buscan refugio. En concreto, se trata de dos campañas creadas en 2016 y 2017, respectivamente. La primera se denominó \#Elviajedesuvida y trataba de hacer reflexionar sobre la situación de los niños que buscan refugio a través de la empatía; la segunda, \#Rompeelmuro, pretendía concienciar sobre los prejuicios y estereotipos que sufre el colectivo de refugiados al llegar a Europa.

Considero de gran relevancia el hecho de poder reflexionar sobre qué tipo de comunicación eligen actualmente las ONGD -en este caso UNICEF- en relación con temáticas que forman parte sustancial de la actualidad y repercuten en nuestro compromiso social colectivo. Dependiendo de los mensajes e información que las ONGD transmitan a la sociedad, esta puede tener una perspectiva y una posición determinante como actor social.

\section{Objetivos}

El objetivo principal de este análisis es esclarecer el tipo de comunicación que escoge UNICEF a la hora de abordar una temática tan actual e importante a nivel humano y social como la situación de las personas que buscan refugio, así como de las personas refugiadas.

Para lograr este objetivo, he postulado los siguientes objetivos específicos: 
- Analizar los estereotipos que se difunden a través de las campañas sobre el colectivo de refugiados.

- Detectar el tipo de información que ofrece UNICEF: comunicación institucional, periodística, sensacionalista, etc.

- Examinar la contextualización de la información que UNICEF brinda en ambas campañas y en su página web.

- Analizar el uso de elementos técnicos.

- Analizar y debatir el papel del público objetivo a través de la estrategia comunicativa de UNICEF (sujeto activo o pasivo).

- Examinar las diferencias y similitudes comunicativas entre los vídeos de las campañas, así como la información que la organización ofrece sobre dichas campañas en su página web.

- Interpretar la comunicación de UNICEF en términos de comunicación transformadora o para el cambio social.

\section{Metodología}

El objeto de estudio de este análisis comparativo son dos campañas realizadas por la ONGD UNICEF. Esta se define a sí misma como «la primera organización internacional en defensa de los derechos de la infancia» (UNICEF, 2016) y trabaja por «un mundo en el que todos los niños y niñas, sea cual sea su procedencia, tengan una vida saludable, vayan al colegio y puedan crecer protegidos de la violencia y la explotación» (UNICEF, 2016).

Así pues, UNICEF lanzó en 2016 una campaña con el lema \#Elviajedesuvida y la intención de «proponer una reflexión sobre la situación de millones de niños y niñas migrantes y refugiados en todo el mundo» (UNICEF, 2016). La campaña cuenta con un vídeo y un micrositio web en el que se profundiza un poco más con datos, testimonios y una imagen interactiva en la que se explica el proceso por el cual pasan las personas refugiadas. "La pieza central de acción de la campaña, que persigue generar empatía con respecto a los millones de niños y niñas migrantes y refugiados, es un vídeo en el que se hace creer a los clientes de una conocida tienda que han sido agraciados con un "maravilloso" viaje que no es lo que parece» (UNICEF, 2016).

Por su parte, en el 2017, UNICEF creó otra campaña con el lema \#Rompeelmuro para "hacer una llamada a derribar los prejuicios y estereotipos a los que se enfrentan miles de niños y niñas migrantes y refugiados durante su camino y llegada a Europa» (UNICEF, 2017). Esta campaña también se complementaba con un micrositio web con testimonios de jóvenes extranjeros que viven en España y cuentan cómo fue su experiencia, así como los obstáculos que enfrentaron. 
Por su parte, el principal activo de la campaña, el vídeo, trata de «un experimento social que recoge reacciones espontáneas de ciudadanos españoles a los que les pidieron que enviaran un mensaje a Obasi, un niño que huye de la guerra (interpretado por el actor Jean Yves Thiemele). Posteriormente, Obasi «atraviesa el muro» para abrazar a aquellas personas que le estaban enviando un mensaje» (UNICEF, 2017).

Para realizar el análisis comparativo de las campañas \#Rompeelmuro y \#Elviajedesuvida de UNICEF, me basaré en el Vademécum para una información internacional responsable de Devreporter Network. Este decálogo pertenece a un proyecto que surgió ante los nuevos retos de desarrollo y solidaridad internacional, para «ayudar a garantizar el derecho de la ciudadanía europea a estar bien informada sobre la realidad internacional y los problemas globales, y de utilizar la información vinculada a la cooperación y el desarrollo para conseguirlo». (Devreporter, 2016).

Devreporter se creó después de realizar múltiples actividades de análisis, formación y debate durante tres años de proyecto, así como de revisar los códigos éticos elaborados por las ONG a escala europea y los códigos deontológicos de periodistas y profesionales de la información (Devreporter, 2016).

Los criterios que Devreporter considera necesarios para una buena práctica comunicativa son los siguientes:ç

1. Favorecer el "periodismo de soluciones».

2. Tratar todos los países y comunidades con dignidad. Evitar el sensacionalismo y la victimización.

3. Diferenciar la información periodística de la comunicación institucional de las organizaciones.

4. Mejorar la visibilidad de los actores de los países del sur implicados $o$ no en las acciones de cooperación internacional e incluidos en la producción de información.

5. Favorecer miradas amplias sobre los países del sur: evitar la oenegización de la información internacional.

6. Favorecer la comprensión de los hechos y su complejidad, aportando contexto y causas.

7. Promover que la realidad internacional se aborde desde los medios de comunicación locales para favorecer el conocimiento mutuo.

8. Tomar perspectiva y cubrir los hechos desde su dimensión global para favorecer la comprensión de las interdependencias presentes y futuras.

9. Hablar de cooperación internacional desde una visión general que permita tener en cuenta los diversos 
aspectos y diferenciar entre acciones de desarrollo y de emergencia.

10. Favorecer el periodismo de seguimiento de acontecimientos.

Así pues, estos van a ser los diez criterios que he utilizado para analizar la comunicación de las campañas de UNICEF. Además, en sintonía con estos criterios, también he tenido en cuenta algunos de los conceptos ya mencionados: advocacy, eficacia cultural, interseccionalidad, sinceridad comunicativa, participación activa, solidaridad activa, performatividad y discursos alternativos, entre otros.

Por otro lado, cabe aclarar que, aun aplicando los mismos criterios, el análisis de los vídeos tiene una naturaleza simbólica por las características de su propio formato.

\section{Resultados}

Tabla 1. Comparación entre los vídeos de las campañas \#Rompeelmuro y \#Elviajedesuvida

\begin{tabular}{|c|c|c|}
\hline CRITERIOS & \#Rompeelmuro & \#Elviajedesuvida \\
\hline 1 & sí & NO \\
\hline 2 & NO & NO \\
\hline 3 & sí & sí \\
\hline 4 & NO & NO \\
\hline 5 & sí & NO \\
\hline 6 & NO & sí \\
\hline 7 & sí & sí \\
\hline 8 & sí & sí \\
\hline 9 & sí & sí \\
\hline 10 & NO & NO \\
\hline
\end{tabular}


Tabla 2. Comparación entre la información del sitio web de UNICEF sobre las campañas \#Rompeelmuro y \#Elviajedesuvida

\begin{tabular}{|c|c|c|}
\hline CRITERIOS & \#Rompeelmuro & \#Elviajedesuvida \\
\hline $\mathbf{1}$ & NO & NO \\
\hline $\mathbf{2}$ & sí & NO \\
\hline $\mathbf{3}$ & NO & sí \\
\hline $\mathbf{4}$ & sí & NO \\
\hline $\mathbf{5}$ & NO & sí \\
\hline $\mathbf{6}$ & NO & NO \\
\hline $\mathbf{7}$ & NO & sí \\
\hline $\mathbf{8}$ & sí & sí \\
\hline $\mathbf{9}$ & sí & NO \\
\hline $\mathbf{1 0}$ & NO & \\
\hline
\end{tabular}

Tabla 3. Comparación entre el vídeo y el sitio web de la campaña \#Rompeelmuro

\begin{tabular}{|c|c|c|}
\hline CRITERIOS & VÍDEO & SITIO WEB \\
\hline 1 & sí & NO \\
\hline 2 & NO & sí \\
\hline 3 & sí & NO \\
\hline 4 & NO & sí \\
\hline 5 & sí & NO \\
\hline 6 & NO & NO \\
\hline 7 & sí & NO \\
\hline 8 & sí & sí \\
\hline 9 & sí & sí \\
\hline 10 & NO & NO \\
\hline
\end{tabular}


Tabla 4. Comparación entre el vídeo y el sitio web de la campaña \#Elviajedesuvida

\begin{tabular}{|c|c|c|}
\hline CRITERIOS & VÍDEO & SITIO WEB \\
\hline $\mathbf{1}$ & NO & NO \\
\hline $\mathbf{2}$ & NO & NO \\
\hline $\mathbf{3}$ & SÍ & sí \\
\hline $\mathbf{4}$ & NO & NO \\
\hline $\mathbf{5}$ & NO & sí \\
\hline $\mathbf{6}$ & SÍ & NO \\
\hline $\mathbf{7}$ & SÍ & sí \\
\hline $\mathbf{8}$ & SÍ & sí \\
\hline $\mathbf{9}$ & SÍ & NO \\
\hline $\mathbf{1 0}$ & NO &
\end{tabular}

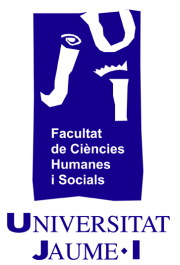

\section{Discusión}

1. Análisis comparativo de los vídeos de \#Rompeelmuro y \#Elviajedesuvida

En términos generales, los puntos fuertes del vídeo de \#Rompeelmuro radican en que ofrece una participación activa de la ciudadanía a través de un experimento social que incita a que las personas se interesen más sobre los prejuicios y barreras de los niños y niñas refugiadas al llegar a España. Por el contrario, no ofrece herramientas para afrontar esos prejuicios, pero sí ofrece la posibilidad de tomar conciencia y cambiar la actitud hacia el colectivo de refugiados a través de la empatía. En ese sentido, en relación con el primer criterio de periodismo de soluciones, el vídeo sí cumple con el «compromiso de generar una mirada crítica para contribuir a la reflexión» (Devreporter, 2016). Sin embargo, el vídeo de la campaña \#Elviajedetuvida, a pesar de que también realiza un experimento social, no va más allá de la descripción visual del problema sin generar una posible conciencia crítica hacia la solución. El vídeo genera empatía, pero no ofrece qué hacer con en ese sentimiento. Un punto muy negativo de esta pieza audiovisual es que genera un sentimiento de frustración y hasta culpabilidad en las personas que participan en el experimento, y tampoco les ofrece las herramientas para gestionar esas emociones.

En cuanto al segundo criterio, ambos vídeos caen en la victimización y el sensacionalismo en alguna ocasión. \#Rompeelmuro 
tiene como imagen principal a Obasi con la cara sucia, el rostro serio

y la mirada perdida. Por su parte, \#Elviajedetuvida muestra imágenes de niños y niñas en situación de vulnerabilidad propia de la caracterización como víctimas. Además, en ambos vídeos se hace uso de palabras tales como "ayuda» o "pena» en varias ocasiones. Por último, la música utilizada genera una sensación de tristeza o compasión.

En tercer lugar, ambos dan información sobre la situación que refleja el vídeo sin mostrar en ningún momento las funciones o actividades que hacen como organización, ni tampoco aparece ningún agente de UNICEF en ellos. Enlaza de este modo con el tercer criterio del Devreporter: «las organizaciones deben ofrecer información más allá de la comunicación vinculada únicamente a sus actividades». En cambio, ninguno de los vídeos cumple con el cuarto criterio que supone «mejorar la visibilidad de los países del sur implicados», pues en ningún caso se da voz a las personas refugiadas o migrantes que aparecen en el vídeo, sino que el protagonismo recae en los espectadores; en este caso, la población civil española que aparece en la pieza audiovisual.

En quinto lugar, el vídeo \#Rompeelmuro sí que cumple con el criterio de favorecer una mirada más amplia de los países del sur, ya que aborda la cooperación desde una vertiente más cultural, teniendo en cuenta la causa y ofreciendo una perspectiva como herramienta de participación. No obstante, el proyecto audiovisual \#Elviajedesuvida tiende a la oenegización de la información.

En lo referente al criterio número seis, el vídeo \#Elviajedesuvida lo cumple en parte, dado que aporta información sobre el contexto y el lugar donde se desarrolla el proyecto (a través de las imágenes que se muestran dentro del vídeo). Cabe puntualizar que ninguna de las dos piezas audiovisuales ofrece un contexto amplio sobre los acontecimientos, pero se ha de tener en cuenta que el objetivo de ambos vídeos no es ofrecer información, sino captar la atención o generar participación.

Los criterios siete, ocho y nueve se cumplen en ambos vídeos. Las dos piezas audiovisuales fomentan el conocimiento de las problemáticas desde un espacio práctico y local, pues ambas se ruedan en Madrid con gente de allí; además los dos vídeos han tenido impacto social en medios de comunicación nacionales y locales. También cumplen con la premisa de «despertar el interés y la curiosidad del público, a través de exponer los problemas comunes y el compromiso de la ciudadanía en su diversidad» (Devreporter, 2016), ya que tanto en los participantes del vídeo como en los espectadores se genera un interés, curiosidad y atención a la diversidad en la situación propuesta en cada vídeo.

En cuanto al criterio ocho, ambas campañas han sabido difundir sin simplificar la problemática, evitando el vocabulario técnico y 
buscando la sencillez sin caer en la simplificación (Devreporter, 2016).

Por último, en ninguno de los dos casos se ofrece un seguimiento de los acontecimientos. Aun teniendo en cuenta que los objetivos no se orientan a ofrecer una continuidad, sí podrían brindar esta posibilidad en vídeos subsiguientes sobre la misma temática o a través del mismo actor, en el caso del vídeo \#Rompeelmuro.

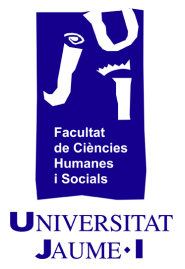

\section{Análisis comparativo de los sitios web de las campañas \#Rompeelmuro y \#Elviajedesuvida}

En primer lugar, ninguna de los micrositios web cumple con el compromiso de generar soluciones, pues ambos espacios se limitan a exponer la problemática y los objetivos con los que trabaja UNICEF en referencia a la campaña, pero no exponen cómo están trabajando para lograr esos objetivos ni qué puede hacer la ciudadanía civil más allá de hacerse socio o aportar una donación.

En cuanto al segundo criterio, a pesar de que en el micrositio web de \#Rompeelmuro sigue apareciendo la foto de Obasi con las características descritas previamente, dentro del mismo espacio aparecen otros testimonios de personas extranjeras que viven en España, a las que se muestra como personas empoderadas y luchadoras. No obstante, el micrositio web de \#Elviajedesuvida ofrece cifras e información sobre la situación de los niños refugiados a través de imágenes en las que se puede observar una victimización clara del colectivo.

En relación con el tercer criterio, ninguno de los dos espacios ofrece información útil para la ciudadanía o los periodistas; tampoco aporta mucho más allá de la comunicación vinculada a sus actividades. En el caso de \#Rompeelmuro, únicamente informa sobre lo que hace UNICEF, mientras que en el sitio web de \#Elviajedesuvida, a pesar de que ofrece datos y cifras, son los mismos que aparecen en el vídeo, por lo que no amplía nada.

Uno de los puntos fuertes de ambos micrositios web y se corresponde con el criterio cuarto es que ambos espacios mejoran la visibilidad de los actores implicados, dándoles voz para contar sus historias a través de los vídeos. Sin embargo, el sitio web de \#Rompeelmuro les da mucha más importancia, ya que los sitúa más arriba $y$ en un tamaño mayor que el micrositio web de \#Elviajedesuvida, donde aparecen al final del todo en un espacio mucho más reducido.

En ningún caso se ofrece una mirada más amplia sobre los países afectados ni, desde luego, se abordan las cuestiones de fondo, por lo que no cumplen con el quinto criterio. Sin embargo, el micrositio web de \#Elviajedesuvida sí cumple con el sexto criterio, dado que ofrece la comprensión de los hechos aportando contexto a través de 
una imagen interactiva en la que explica cómo es el proceso de un refugiado y las dificultades a las que se enfrenta.

En cuanto al criterio número siete, ambos vídeos coinciden en no aportar conocimiento sobre las problemáticas e intereses locales, así como tampoco hacen propuestas adaptadas al periodismo de proximidad. En contrapunto, los dos sitios web consiguen captar la atención del público a través de imágenes, vídeos e información dinámica. Además, no caen en la simplificación del problema y lo transmiten de una forma sencilla y adaptada para todos los públicos. De esta forma cumplen con los criterios octavo y noveno.

Por último, ninguna de las dos campañas aporta información ni realiza un seguimiento de los acontecimientos o su evolución. Así pues, no cumplen con el criterio número diez.

\section{Análisis comparativo entre el vídeo y el sitio web de la campaña \#Rompeelmuro}

Aunque se supone que los micrositios web y los vídeos se complementan mutuamente, al analizar ambos formatos se puede observar que esa complementación se da en muy pocos criterios. Así pues, llama la atención que el vídeo sí ofrece de manera simbólica la capacidad de respuesta hacia una solución, mientras que el sitio web se queda en la exposición de situación y experiencias. En ese sentido, ocurre lo mismo con el hecho de abordar las cuestiones desde diferentes perspectivas y no solo desde los proyectos de la organización: el vídeo no muestra nada de la organización y se centra en un enfoque más sociocultural de la cooperación y solidaridad internacional, mientras que el micrositio web solo ofrece información sobre los objetivos y acciones de la organización. En el resto de los criterios, que previamente ya han sido analizados, ambos formatos coinciden ya sea de forma negativa o positiva.

\section{Análisis comparativo entre el vídeo y el sitio web de la campaña \#Elviajedesuvida}

En esta ocasión, las diferencias entre ambos formatos son mayores. En primer lugar, en relación con el tercer criterio, la información que se ofrece más allá de la comunicación de las actividades de la organización es la misma tanto en el vídeo como en el micrositio web; este último sirve de espacio para ampliar información. En contraste, el sitio web sí da visibilidad y voz a los actores implicados, mientras que la pieza audiovisual lo hace con la sociedad civil española (cuarto criterio).

Por otro lado, el vídeo incluye espacio para la práctica local (dado que en sí mismo se basa en una práctica local), al tiempo que ofrece la posibilidad de adaptarse a la difusión desde el periodismo de proximidad; el micrositio web, sin embargo, no ofrece ninguna de 
las dos posibilidades anteriores, dado que se limita a exponer la información del vídeo y testimonios.

\section{Conclusiones}

En líneas generales y tras realizar el análisis de las dos campañas, una de las conclusiones a las que he podido llegar es que ninguna de ellas encarna una verdadera plataforma de información contextualizada que permita a la sociedad civil conocer cuáles son las causas de fondo, los procedimientos, los efectos y las consecuencias de la situación de las personas refugiadas. En ese sentido, considero positivo el hecho de que en ninguno de los dos espacios, incluidos los vídeos, aparece el botón interactivo de "Dona» o "Hazte socio», dándole así más importancia al hecho de informar, difundir y dar a conocer los contenidos de las campañas. A pesar de ello, tras ver todo el contenido de los sitios web y en el caso de que el lector quiera obtener más información, el propio botón que ofrece esta posibilidad deriva directamente a la página para donar o hacerse socio. Esto coincide con el hecho de que ninguna de las dos campañas aclara cómo se puede participar de forma activa en la situación propuesta, más allá de las donaciones.

Por otro lado, ambas campañas apuestan por dar voz a los actores implicados, incluyendo varios vídeos en los sitios web en los que personas extranjeras cuentan su historia como migrantes o refugiados. Un aspecto muy positivo es que los vídeos están encaminados a ofrecer una imagen empoderada de las personas que relatan su experiencia. Sin embargo, las imágenes de la campaña \#Elviajedetuvida sí caracteriza como víctimas a las personas que aparecen.

Cabe tener en cuenta que este análisis no puede mostrar una visión amplia de la comunicación que realizan las ONGD, ni tan siquiera sobre la comunicación global de UNICEF. Esto se debe a que, por ejemplo, la página principal de esta organización se limita a pedir una donación mediante una imagen en la que una niña refugiada se muestra claramente como víctima al lado de un trabajador de UNICEF, potenciando así la imagen de salvador de este último. Se genera así un choque contradictorio entre las líneas de trabajo de las campañas analizadas y otros espacios de la propia organización.

Aun así, considero que a través de este análisis se puede ver cómo UNICEF empieza a dejar de lado la idea (al menos visualmente) del eje víctima-salvador, a pesar de que todavía tiene mucho que mejorar en términos de eficacia cultural y solidaridad participativa. 
ACNUR (Alto Comisionado de las Naciones Unidas para los Refugiados). 1950. Convención sobre el Estatuto de los Refugiados. Acta de la Conferencia de Plenipotenciarios sobre el Estatuto de los Refugiados y de los Apátridas. Resolución Asamblea General 429 (V). Acceso del 25 de octubre de 2017 http://www.acnur.org/fileadmin/scripts/doc.php?file=fileadmin/ Documentos/BDL/2001/0005.

- 1967. Protocolo sobre el Estatuto de los Refugiados, Asamblea General resolución 2198 (XXI). Acceso del 26 de octubre de 2017 http://www.acnur.org/fileadmin/scripts/doc.php?file=fileadmin/ Documentos/BDL/2001/0003

-. 2016. El desplazamiento forzado alcanza un nuevo récord. Acceso del 24 de octubre de 2017 http://www.acnur.org/recursos/estadisticas/.

CEAR (Comisión Española de Ayuda al Refugiado). 2017. Las personas refugiadas en España y Europa (15). Acceso https://www.cear.es/wp-content/uploads/2017/06/InformeAnual-CEAR-2017.pdf

Devreporter. 2016. Vademécum para una información internacional responsable. Acceso http://devreporternetwork.eu/wpcontent/uploads/2016/04/vademecum_DR_ESP.pdf

Erro, Javier y Tere Burgui. 2011. "Comunicando para la solidaridad y la cooperación: cómo salir de la encrucijada». Pamplona: Foro Comunicación, Educación y Ciudadanía.

García López, Marcial. 2013. «Repensar la comunicación para la paz y la solidaridad desde lo participativo». Razón y palabra 81. http://www.razonypalabra.org.mx/N/N81/M81/10_Garcia_M81 .pdf.

Nos Aldás, Eloísa, Eduardo A. Sandoval Forero y Alex I. Arévalo Salinas. 2012. Migraciones y cultura de paz. Educando y comunicando solidaridad. Madrid: Dykinson.

Nos Aldás, Eloísa, Amador Iranzo y Alessandra Farné. 2012. "La eficacia cultural de la comunicación de las ONGD: los discursos de los movimientos sociales actuales como revisión». CIC: Cuadernos de Información y Comunicación 17: 209-237.

UNICEF. s.d. «Protege a los niños del duro invierno». Unicef. Acceso https://www.unicef.es/hazte-socio-refugiados-invierno.

UNICEF. s.d. «Rompe el muro». Unicef.

Acceso https://www.unicef.es/rompe-el-muro

UNICEF. s.d. «El viaje de su vida». Acceso http://elviajedesuvida.es 


\section{La eficacia cultural en la comunicación de las ONGD:}

Leyre Navarrete Emmanuel

leyrenavarrete@gmail.com 
I. Resumen

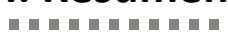

Ante la diversidad y pluralidad de comunicaciones por parte de las ONGD hoy en día, es importante recordar la particularidad del tercer sector y la responsabilidad de estas organizaciones como interlocutores sociales que fomenten la sensibilización de la ciudadanía, su educación, la lucha por la justicia social y contra las relaciones de poder, tanto local como globalmente. Tratándose de actores con responsabilidad social ante la ciudadanía, es necesario que sus discursos estén guiados por dichos valores sin caer en la contradicción de que la necesidad recaudatoria suponga perpetuar estereotipos y situaciones generadoras de desigualdad que ellas mismas luchan por eliminar.

Habiendo encarnado un aparente giro en los discursos tradicionales de las ONGD, la campaña \#CierraUnicef se analiza en este artículo con una mirada constructiva, utilizando como método de análisis el decálogo del Vademécum para una Información Internacional Responsable, del proyecto DEVREPORTER, con el objetivo de localizar posibles elementos generadores de desigualdad en las relaciones de cooperación, así como avances positivos en dicha dirección que faciliten el camino hacia una comunicación desde las ONGD más inclusiva, empoderadora y respetuosa sobre la que seguir construyendo.

Si bien esta comunicación cuenta con ciertas elecciones narrativas que cumplen algunos criterios de ese decálogo, vemos cómo detrás de esa narración se incluyen elementos que perpetúan los marcos tradicionales de la cooperación basados en la desigualdad, en los que el asistencialismo rige las relaciones nortesur y en las que se presenta a las ONGD como salvadoras, portadoras de soluciones inalcanzables por unos protagonistas incapaces $y$ estáticos, que dependen en gran medida de las ONGD para avanzar.

Palabras clave: comunicación, ONGD, tercer sector, eficacia cultural, advocacy, cambio social

\section{Introducción}

Este artículo tiene como objetivo el análisis de la comunicación llevada a cabo desde Unicef en su campaña \#CierraUnicef de 2017 desde una perspectiva de eficacia cultural, que aborde la cooperación y el desarrollo desde discursos libres de estereotipos que respeten la dignidad de sus protagonistas.

La diversidad y pluralidad de ONGD (concepto amplio y genérico pero que en adelante usaré, dado que es el más extendido, al referirme al tercer sector estructurado de cooperación) es una 
realidad muchas veces abrumadora hoy en día. La comunicación de las ONGD forma parte cada vez más de nuestro entorno, en el que los medios de comunicación nos acercan su mensaje y, si bien es cierto que cada una de ellas aborda la cooperación desde un enfoque y estrategias propios, es innegable que en la actualidad existen numerosos casos en los que el afán recaudatorio y estrategias que recuerdan al más puro marketing han desplazado la eficacia cultural de su discurso.

Partiendo de esa realidad plural de ONGD que comentamos, se hace imprescindible abordar las peculiaridades y responsabilidades que diferencian al tercer sector de otros sectores sociales, como las empresas o el Estado. Como Nos Aldás explica, estas organizaciones cuentan con unos objetivos de trabajo colectivo orientados al bien público y unas formas de trabajo cooperativas, por y desde la solidaridad (2007: 210-212). Esas características rigen -o deberían regir - el trabajo y la visión de estas organizaciones, que como actores sociales fundamental para la concienciación de las desigualdades globales, cuentan con responsabilidades comunicativas que parten de responsabilidades educativas y de incidencia política por la justicia social y la erradicación de la pobreza (FCONGD, 1997; CONGDE, 1998).

Así, los propósitos de las ONGD están íntimamente ligados al concepto de advocacy (utilizado en inglés dada su complejidad de traducción al español por falta de un término concreto), que combina una «responsabilidad de sensibilización más una pedagogía política y cultural y una incidencia política, legislativa y cultural hacia procesos de cambio social» (Nos Aldás et al., 2012: 212). En estas organizaciones recae, por lo tanto, una responsabilidad comunicativa muy importante: al carácter específico de sus valores y objetivos se une la gran incidencia que sus comunicaciones tienen en la ciudadanía. Las realidades y las problemáticas que las campañas de las ONGD reflejan a menudo retratan otras totalmente ajenas a las del público al que se dirigen, en cuyo frenético día atisban de pasada lo que puede estar sufriendo alguien al otro lado del mundo a través de la marquesina de la parada de autobús, el anuncio de televisión entre programa y programa, o la foto o vídeo de Facebook que aparece en su muro cuando abre la red social.

Uno de los principales retos de la comunicación de las ONGD y el que se erige como su horizonte es la eficacia cultural, desde la que localizar la violencia cultural existente en los discursos, y ver cómo transformar esa violencia en cultura de paz. Se trata de una comunicación para el cambio social desde el advocacy (Nos Aldás et al., 2012), que sensibilice, forme y eduque, sin caer en los estereotipos tan habituales del discurso hegemónico actual, y promueva una comunicación horizontal. 
Esta eficacia cultural parte de la performatividad del discurso (Austin, 1976) y su acción comunicativa (Habermas, 1987) que puede traducirse en la implicación social a partir de las comunicaciones de las ONGD desde su transversalidad (Nos Aldás et al., 2012: 214). La importancia de la eficacia cultural de la comunicación de las ONGD deriva a su vez de la indiscutible influencia de sus discursos en la construcción de la realidad, en este caso del desarrollo y la cooperación, por parte de los que los reciben. Cada una de las elecciones que se hacen a la hora de comunicar (lenguaje, imágenes, protagonistas, perspectiva de enfoque, etc.) se traduce en «la concepción que el emisor tiene de la realidad de la que habla, su relación con esas realidades y la reacción que busca en los públicos. Cada mensaje no solo realiza una propuesta de solidaridad determinada, sino que refleja la solidaridad del emisor y de su propia comunicación» (Nos Aldás, 2010: 15).

Sin embargo, la realidad es que somos testigos y receptores de comunicaciones de ONGD en las que el enfoque asistencialista presenta a sus protagonistas como víctimas incapaces, inmóviles, sin contexto ni historia, que necesitan de nuestra "caridad" y la de las ONGD para mejorar su vida. A su vez, la implicación de la ciudadanía se hace desde una relación vertical en la que se asume que su único poder es el monetario. El trabajo de Nos, Iranzo y Farné expone esta realidad actual de las ONGD, en la que se plasma esta crisis de valores y tras la que se hace necesario que las ONGD no sigan promoviéndose como "catalizadoras y movilizadoras de recursos [...] para asumir como función principal la de agentes de cambio» (De Souza, 2009: 701; Santolino, 2010). Se hace necesario cambiar esa "solidaridad del mínimo esfuerzo" fomentada por las ONGD (Erro Sala 2000; 2003a; 2003b; 2006, entre otros; Ballesteros 2002; Rizzardini 2002).

$\mathrm{Si}$ pretendemos mejorar la eficacia cultural de esas comunicaciones, los compromisos que la ciudadanía y los poderes públicos adopten, y romper con los estereotipos y las relaciones de poder que justifican a la vez que legitiman la desigualdad, necesariamente debemos cambiar el marco (framing) desde el que se comunica, así como abandonar los marcos que han promovido y mantenido esta situación. George Lakoff en su teoría de los marcos los define de la siguiente manera:

las estructuras mentales que nos permiten a los seres humanos comprender la realidad $y$, en ocasiones, crear lo que nosotros consideramos que es la realidad (...). Estructuran nuestras ideas y nuestros conceptos, conforman nuestra manera de razonar e incluso repercuten en la manera en la que percibimos y actuamos. La mayoría de las veces usamos los marcos de forma inconsciente y 
automática; los usamos sin darnos cuenta. (2006: 61 citado en Darton

y Kirk 2011, y Mesa 2013).

Los marcos son la manera que tenemos de ver, explicar y dar sentido al mundo. Sin embargo, para cambiar esos marcos no basta con ir contra ellos. En su trabajo Visibles y transgresoras, Mesa desarrolla el funcionamiento de esos marcos, partiendo de las aportaciones de Lakoff, y los retos necesarios para su cambio efectivo. Si queremos cambiar los marcos que han recibido los ciudadanos por diversas fuentes, entre ellas las ONGD, y que han contribuido a la construcción de estereotipos y la asignación de roles, no basta con la crítica a esos mismos marcos. Es necesario un cambio de lenguaje y la construcción de marcos alternativos, ya que su denuncia refuerza el marco y la utilización de su mismo lenguaje vuelve a remitirnos a él, haciéndolo más fuerte (Lakoff, 2007; Mesa, 2013). "Cambiar el marco significa promover el cambio social. Cambiar el marco es cambiar el modo que tiene la gente de ver el mundo. Es cambiar lo que se entiende por sentido común». (Lakoff, 2007, citado en Mesa, 2013).

Mesa (2013) promueve la construcción de marcos alternativos, y aunque su trabajo se enfoca a las relaciones de desigualdad entre hombres y mujeres, esos marcos propuestos sin duda nos son útiles para aplicar esos marcos alternativos en el caso específico de las relaciones de desigualdad en el ámbito del desarrollo y la cooperación (desigualdades norte-sur, países desarrollados frente a países subdesarrollados). Los marcos tradicionales de mujer víctima frente a hombre héroe tienen una relación directa indudable en las relaciones de poder de hombre blanco poderoso y salvador, en contraste con persona de color pobre, desvalida e incapaz. A partir de estos marcos alternativos propuestos, defendemos un retrato de las situaciones que no aísle de la realidad, que dé voz a sus protagonistas y que los empodere exponiéndolos en su contexto. Es necesario abandonar los discursos en los que se priva de voz al protagonista representándolo en situaciones que incitan a la evocación de emociones como la pena o la culpa (como, por ejemplo, un niño de África, retratado solo, sin contexto y con el vientre abultado).

Los prejuicios y estereotipos que atribuyen rasgos y roles también se han apoderado de los discursos de cooperación, en los que se discrimina a sus propios protagonistas. Es preciso un cambio en la denuncia, que ponga el foco en las causas y los responsables que propician esa violencia o desigualdad en lugar de en las víctimas, para evitar que esa sobrerrepresentación de diversos colectivos como víctimas fomente una visión estereotipada y dicotómica (Mesa, 2013: 31) que ha construido el enfoque asistencialista imperante. Un marco que, como el propuesto de «mujeres visibles y transgresoras», 
"se sustente en estructuras sociales horizontales, abiertas y democráticas, con relaciones basadas en redes que conectan personas activando valores de equidad, colectividad, dinamismo, creatividad, resiliencia y resistencia. Se trata de deslegitimar la lógica de la violencia que pone en juego la vida de los seres humanos para perseguir intereses materiales, ideológicos, de poder o soberanía». (Mesa 2013: 34, a partir de Magallón, 2012a).

Dada la particularidad del tercer sector, debemos recordar la responsabilidad de las ONGD como interlocutores sociales que fomenten la sensibilización de la ciudadanía, su educación, la lucha por la justicia social y contra las relaciones de poder, tanto local como globalmente. Por ello, así como por la relación y efecto directos que tienen en la ciudadanía incidiendo en la forma en que percibe esas realidades tan a menudo lejanas, es importante que sus discursos estén guiados por dichos valores sociales sin caer en que la necesidad recaudatoria por su supervivencia económica siga perpetuando estereotipos y situaciones generadoras de desigualdad.

A partir de esta problemática, el presente artículo tiene el objetivo de localizar en esta campaña, con una mirada constructiva, los posibles elementos perpetuadores de desigualdad en las relaciones de cooperación a través de su comunicación, así como avances positivos que faciliten el camino hacia una comunicación desde las ONGD más inclusiva, empoderadora y respetuosa sobre la que seguir construyendo, al tiempo que tratamos de contestar a la pregunta de si cumplen las campañas de comunicación de las ONGD los criterios para una información internacional responsable.

\section{Objetivos}

Las ONGD actúan a menudo como ventana de la sociedad a problemas del mundo que nos es difícil conocer de otra manera. Por ello, se debe prestar especial atención a la comunicación de estas organizaciones, dado su papel significativo en la transformación social y en la educación de una ciudadanía global crítica, como defienden Nos, Iranzo y Farné (2012). De esta manera, el análisis de la campaña de \#CierraUnicef se abordará desde una mirada de comunicación para el cambio social y transformadora.

En el reto de la comunicación de las ONGD con el horizonte y guía de la eficacia cultural, se hace imprescindible identificar las violencias existentes en los discursos para poder emprender el camino hacia la cultura de paz mediante el cambio social, localizando los estereotipos perpetuadores de desigualdad.

En suma, el objetivo de este trabajo es el de localizar, a través del análisis de la comunicación de la campaña elegida, elementos generadores de desigualdad en las relaciones de cooperación o que no cumplan con los criterios para una información internacional 
responsable, así como aquellos que sí lo hagan, para poder proponer

alternativas específicas para una comunicación horizontal, responsable y eficaz culturalmente desde las ONGD.

\section{Material y método}

El objeto de estudio de este análisis es la comunicación de la campaña \#CierraUnicef del Comité Español de UNICEF (Fondo de las Naciones Unidas para la Infancia), tomando como objeto específico el vídeo de la campaña incluido en su página web, que se difundió por los medios de comunicación.

La muestra se ha elegido por tratarse de una comunicación por parte de una ONGD con cierto giro en el discurso, sin repetir fórmulas a las que la ciudadanía está acostumbrada, y que merece ser analizada tanto por su novedad como por su ambigüedad.

Como método de análisis cualitativo se utilizará el decálogo del Vademécum para una información internacional responsable del proyecto Devreporter. Se contrastará con la campaña para ver en qué medida cumple con sus principios.

\section{Resultados}

Una vez realizado el análisis cualitativo del vídeo de esta campaña a través del Vademécum del Devreporter para observar si se cumplen estos diez principios, los resultados para cada uno de esos principios son los siguientes:

\begin{tabular}{|c|c|c|}
\hline Principio del Vademécum & Ideas principales & Cumplimiento \\
\hline $\begin{array}{ll}1 . \quad \text { Favorecer } & \text { el } \\
\text { "periodismo } & \text { de } \\
\text { soluciones" } & \end{array}$ & $\begin{array}{l}\text { - posibles soluciones de los } \\
\text { diferentes actores } \\
\text { - propuestas sociedad civil } \\
\text { - mirada crítica que contribuya } \\
\text { a la reflexión }\end{array}$ & $\begin{array}{l}- \text { No } \\
- \text { No } \\
\text { - No }\end{array}$ \\
\hline $\begin{array}{l}\text { 2. Tratar todos los países } \\
\text { y comunidades con } \\
\text { dignidad. Evitar el } \\
\text { sensacionalismo y la } \\
\text { victimización }\end{array}$ & $\begin{array}{l}\text { - Importancia de vocabulario, } \\
\text { lenguaje escrito y audiovisual } \\
\text { - Evitar lenguaje estereotipado, } \\
\text { discriminatorio y androcéntrico } \\
\text { - No difundir fotografías que } \\
\text { vulneren la dignidad humana y } \\
\text { de los niños } \\
\text { - Pueblos y comunidades como } \\
\text { luchadoras no solo como } \\
\text { víctimas }\end{array}$ & $\begin{array}{l}\text { - Sí } \\
\text { - No } \\
\text { - Sí } \\
\text { - Sí y no }\end{array}$ \\
\hline $\begin{array}{l}\text { 3. Diferenciar la } \\
\text { información periodística } \\
\text { de la comunicación } \\
\text { institucional de las }\end{array}$ & $\begin{array}{l}\text { - Énfasis en la situación de los } \\
\text { países afectados en vez de } \\
\text { solamente información de la } \\
\text { propia ONG }\end{array}$ & - No \\
\hline
\end{tabular}




\begin{tabular}{|c|c|c|}
\hline organizaciones & & \\
\hline $\begin{array}{l}\text { 4. Mejorar la visibilidad de } \\
\text { los actores de los países } \\
\text { del sur implicados o no en } \\
\text { las acciones de } \\
\text { cooperación internacional } \\
\text { e incluirlos en la } \\
\text { producción } \\
\text { información }\end{array}$ & $\begin{array}{l}\text { - Consultar diversas fuentes, } \\
\text { también del Sur y dar voz a las } \\
\text { poblaciones } \\
\text { - Alianzas norte-sur para } \\
\text { enriquecer, diversificar y } \\
\text { difundir información } \\
\text { - Personas que cuenten sus } \\
\text { propias historias, incluida la } \\
\text { opinión de los niños en lo que } \\
\text { les afecte }\end{array}$ & - No \\
\hline $\begin{array}{l}\text { 5. Favorecer miradas } \\
\text { amplias sobre los países } \\
\text { del sur: evitar la } \\
\text { «oenegización» de la } \\
\text { información internacional }\end{array}$ & $\begin{array}{l}\text { - Abordar cooperación desde } \\
\text { vertientes múltiples } \\
\text { (económica, política y cultural) } \\
\text { - Perspectiva de las acciones de } \\
\text { la solidaridad internacional }\end{array}$ & - No \\
\hline $\begin{array}{l}\text { 6. Favorecer la } \\
\text { comprensión de los } \\
\text { hechos y su complejidad, } \\
\text { aportando contexto y } \\
\text { causas }\end{array}$ & $\begin{array}{l}\text { - Aportar contexto de } \\
\text { acontecimiento y lugar de los } \\
\text { proyectos } \\
\text { - Aporte datos disgregados (por } \\
\text { sexo, edad o etnia) }\end{array}$ & $-\mathrm{No}$ \\
\hline $\begin{array}{l}\text { 7. Promover que la } \\
\text { realidad internacional se } \\
\text { aborde desde los medios } \\
\text { de comunicación locales } \\
\text { para favorecer el } \\
\text { conocimiento mutuo }\end{array}$ & $\begin{array}{l}\text { - Conocer problemáticas e } \\
\text { intereses locales, de proximidad } \\
\text { - Mostrar prácticas locales que } \\
\text { puedan ser inspiración a nivel } \\
\text { internacional }\end{array}$ & - No \\
\hline $\begin{array}{l}\text { 8. Tomar perspectiva y } \\
\text { cubrir los hechos desde su } \\
\text { dimensión global para } \\
\text { favorecer la comprensión } \\
\text { de las interdependencias } \\
\text { presentes y futuras }\end{array}$ & $\begin{array}{l}\text { - Relación entre las causas de } \\
\text { las problemáticas y sus } \\
\text { impactos en diferentes países } \\
\text { del norte y del sur } \\
\text { - Despertar interés y curiosidad } \\
\text { del público, exponiendo los } \\
\text { problemas comunes y el } \\
\text { compromiso de la ciudadanía } \\
\text { en su diversidad }\end{array}$ & - No \\
\hline $\begin{array}{l}\text { 9. Hablar de cooperación } \\
\text { internacional desde una } \\
\text { visión general que } \\
\text { permita tener en cuenta } \\
\text { los diferentes aspectos y } \\
\text { diferenciar acciones de } \\
\text { desarrollo y de } \\
\text { emergencia. }\end{array}$ & $\begin{array}{l}\text { - Evitar vocabulario técnico de } \\
\text { cooperación; sencillez sin caer } \\
\text { en la simplificación } \\
\text { - No hacer presentación } \\
\text { puramente técnica de la } \\
\text { cooperación. No descuidar sus } \\
\text { dimensiones políticas }\end{array}$ & - Sí y no \\
\hline $\begin{array}{lr}10 \quad \text { Favorecer } & \text { el } \\
\text { periodismo de } \\
\text { seguimiento de los } \\
\text { acontecimientos }\end{array}$ & $\begin{array}{l}\text { - Huir de } \begin{array}{l}\text { dictadura de } \\
\text { emergencia, conflicto } \\
\text { desastre }\end{array} \\
\text { - Seguimiento, información } \\
\text { regular }\end{array}$ & - No \\
\hline
\end{tabular}

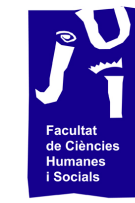

UNIVERSITAT JAUME•I 
Una vez presentados los resultados, apreciamos que esta campaña no cumple con muchas de las premisas para la información internacional responsable. En este vídeo no se están presentando ni el contexto ni las causas de las situaciones, ni tampoco se expone la problemática desde todas sus vertientes (económica, política y social). Por otro lado, no se da una voz real a sus protagonistas para que expresen la situación desde su punto de vista, ni se exponen propuestas provenientes de los países del sur que puedan enriquecer la narrativa y sirvan de inspiración en una cooperación inclusiva.

Todo ello puede tener como consecuencia la perpetuación de los marcos tradicionales de la cooperación en los que el asistencialismo rige las relaciones norte-sur y en los que se presenta a las ONGD como salvadoras, portadoras de soluciones inalcanzables por unos protagonistas incapaces y estáticos, que sin dichas ONGD serían incapaces de seguir adelante.

Un ejemplo de ello es el discurso utilizado al principio del vídeo, en el que la voz de la niña que habla describe a los trabajadores de Unicef que se van -en su gran mayoría blancos- como «[...] quienes sanaban nuestras heridas, quienes prevenían nuestras enfermedades y colmaban de conocimiento nuestras dudas». Esto genera una imagen de los protagonistas como dependientes de la ONGD, sin mostrar la cooperación desde valores de equidad, colectividad y creatividad. No se empodera a sus protagonistas ni se ponen en valor los recursos y las iniciativas que pueden surgir desde ellos.

Otro ejemplo es el escenario en que se graba el vídeo y los únicos protagonistas que se muestran, haciendo referencia a una realidad muy delimitada. Solo se muestra a niños de color en una población de lo que se intuye que es África. No da voz ni muestra realidades del resto de los niños del mundo, de distintas etnias, procedencias o situaciones a los que la ONGD también puede ayudar, perpetuando así el marco estereotipado de África como «necesitada de ayuda». Del mismo modo, no favorece una mirada crítica que contribuya a la reflexión del receptor de la comunicación ni fomenta la idea de sociedad global interconectada, sino más bien de "sociedad jerarquizada».

Por otra parte, la comunicación de este vídeo sí que cumple con otros criterios de este marco de la eficacia cultural: ofrece una representación distinta a la de los protagonistas como víctimas, así como expone una imagen de ellos como seres humanos capaces y luchadores aunque con ciertos matices, ya que, a pesar de que los protagonistas de este vídeo son niños, no se muestra a otros individuos de esas mismas comunidades ocupando escenarios decisivos, como mujeres adultas desarrollando roles distintos a los 
del cuidado del hogar y como protagonistas de la vida política y económica, para así poder transformar el marco-.

También da la voz este vídeo a los niños para que sean ellos los que cuenten su historia, en lugar de hablar solo desde la organización, lo cual ayuda a visibilizar y poner en valor a esos protagonistas transformando el discurso hegemónico en el que la información parte de la voz de la ONGD que funciona como intermediaria. Igualmente, respeta la dignidad humana al no difundir imágenes que puedan vulnerarla, mostrando a los niños protagonistas desde la alegría.

Por último, quizás lo más relevante (a mi juicio) sean las elecciones narrativas en las que se presenta una realidad distinta a la imperante en el imaginario hegemónico relativo a la cooperación, si bien por ahora es solo un imaginario en el que esos protagonistas no necesitan a la organización y esta desaparece de su realidad. Se presenta a la ONGD como prescindible y desde un discurso en el que se desea su desaparición.

Por otro lado, la campaña no está pidiendo dinero basándose en emergencias ni catástrofes, rechazando así ese discurso recurrente por parte de las ONGD que propicia esa «solidaridad del mínimo esfuerzo». El discurso no busca una recaudación motivada por los padecimientos de niños desamparados, sino que pretende un cambio estructural empoderador que suponga que la existencia de esta organización ya no sea necesaria.

Como formas de mejorar hacia una mayor eficacia cultural, si se quiere realmente conseguir ese cambio estructural, hace falta una comunicación que lo facilite, por lo que se debe dar visibilidad a las causas y los contextos tanto de las desigualdades como de las relaciones de poder que las facilitan y perpetúan. Asimismo, es importante ofrecer soluciones y que estas provengan de fuentes diversas, para así empoderar a los que tradicionalmente han sido discriminados y silenciados, convirtiéndolos en actores sociales capaces, creativos y con recursos.

Por último, y en relación con la teoría de los marcos de Lakoff, en el vídeo se parte de la idea del discurso tradicional hegemónico de la superioridad y necesidad de la ONGD, y aunque se ofrece después una visión distinta de esa relación entre ONGD-protagonistas en virtud del giro narrativo, al comunicar desde un marco - aunque sea con el fin de criticarlo-, se corre el riesgo de caer en su refuerzo.

Por ese motivo recomendaría la construcción de un marco de comunicación alternativo en el que no se apele a situaciones tradicionales de desigualdad ni siquiera para desmontarlas, sino que cree una nueva posibilidad de relaciones en las que esas desigualdades y violencias dejen de tener sentido. 
El análisis aquí presentado aborda la cuestión de la eficacia cultural en la comunicación de las ONGD, estudiando el caso concreto de la campaña \#CierraUnicef de Unicef que llevó a cabo durante el año 2017 con la metodología del Vademécum para una información internacional responsable del proyecto Devreporter.

Puesto que la campaña \#CierraUnicef es amplia, tiene ya un recorrido en el tiempo y está formada por muchos tipos de comunicaciones, principalmente su página web, se ha decidido delimitar el objeto de estudio al vídeo de la campaña en concreto. Sin embargo, dejo la línea de investigación de la página web abierta para posibles trabajos futuros, considerando para este caso más apropiado hacerlo mediante la metodología DOCHAS, más enfocada a la comunicación desde las imágenes.

Tras este análisis basado en el Vademécum enmarcado en la metodología de la eficacia cultural, y a pesar de la limitación del objeto de estudio, se puede afirmar que este vídeo enmarcado en esta campaña de \#CierraUnicef sí que recoge ciertos rasgos de la eficacia cultural por presentar un discurso en el que los protagonistas no son retratados meramente como víctimas, además de incapaces e inmóviles, y a través del cual se anima a que las ONGD aspiren a ser prescindibles. Sin embargo, es cierto que no cumple otros aspectos, como el de proporcionar el contexto y las causas de las problemáticas protagonistas en la cooperación, ni da visibilidad ni voz propia a las diversas fuentes y soluciones. Tampoco plasma una lucha conjunta de todas las esferas (incluida la política) para lograr un cambio sustancial y la construcción de un nuevo marco más igualitario.

Por lo tanto, tal y como hemos visto en los apartados anteriores, sería interesante aplicar códigos que lleven a unos planteamientos más eficaces culturalmente a largo plazo, puesto que Unicef es una organización que trabaja desde hace décadas a nivel estructural. Si lo que se pretende es un cambio en este sentido, es necesaria una comunicación que lo facilite.

\section{Bibliografía}

Austin, John Langshaw. 1976. How to do things with words. Oxford: Oxford University Press.

Ballesteros, Carlos. 2002. "Supermercados de la solidaridad». En La ética de las ONG y la lógica mercantil, coordinado por Luis Nieto, 89-134. Barcelona: Icaria. 
de Souza, Rebecca. 2009. "Creating "Communicative Spaces": A Case

of NGO Community Organizing for HIV/AIDS Prevention». Health Communication 24 (8): 692-702

CONGDE (Coordinadora de ONG para el desarrollo, España). 1998. Código de Conducta de las ONG de Desarrollo. Acceso del 15 de diciembre de 2018. https://coordinadoraongd.org/wpcontent/uploads/2016/01/Codigo_Conducta.pdf

Darnton, Andrew y Kirk, Martin. 2011. Finding Frames: New Ways to Engage the UK Public in Global Poverty. Londres: BOND for International Development.

DEVREPORTER. 2012. Vademécum para una información internacional responsable. Acceso el 15 de diciembre de 2018. http://devreporternetwork.eu/wpcontent/uploads/2016/04/vademecum_DR_ESP.pdf

DOCHAS (The Irish Association of Non Governmental Development Organisations). 2006. Code of conduct on images and messages. Acceso del 15 de diciembre de 2018. http://www.dochas.ie/sites/default/files/Images_and_Messages .pdf

Erro Sala, Javier. 2000. «Las prácticas comunicativas de las ONGD. De la comunicación mercadeada a la construcción de una mirada comunicacional». Directorio de ONGD 2000, 51-72. Madrid: CONGDE.

-. 2003a. Descubrir y construir procesos de comunicación social (Herramientas). Bilbao: Hegoa.

-. 2003b. «ONGD: ¿comunicarse por qué y para qué? El paso de la comunicación mercadeada a la comunicación social educativa». en La publicidad en el Tercer Sector. Tendencias y perspectivas de la comunicación solidaria, editado por Vicente J. Benet y Eloísa Nos Aldás, 53-81. Barcelona: Icaria.

-. 2006. «¿Pensar la comunicación o revisar el modelo de organizaciones no gubernamentales para el desarrollo (ONGD)?». En Medios de comunicación y solidaridad: reflexiones en torno a la (des)articulación social, editado por Eloísa Nos Aldás y María José Gámez Fuentes, 89-106. Castellón: Servei de Publicacions de la Universitat Jaume I.

FCONGD (Federación Catalana de ONGD). 1997. Código ético y de conducta. Barcelona: FCONGD.

Habermas, Jürgen. 1987. Teoría de la acción comunicativa. Madrid: Taurus.

Lakoff, George. 2007. No pienses en un elefante: lenguaje y debate político. Madrid: Editorial Complutense. 
Magallón, Carmen. 2012. Contar el mundo. Una mirada sobre las relaciones internacionales desde la vida de las mujeres. Madrid: Cuadernos inacabados. Horas y horas.

Mesa, Manuela. 2013. Visibles y transgresoras: narrativas y propuestas visuales para la paz y la igualdad. Fundación Cultura y Paz.

Nos Aldás, Eloísa, ed. 2003. La publicidad en el tercer sector. Tendencias y perspectivas de la comunicación solidaria. Barcelona: Icaria.

-. 2007. Lenguaje publicitario y discursos solidarios. Eficacia publicitaria, ¿eficacia cultural? Barcelona: Icaria.

—. 2010. «Comunicación, cultura y educación para la solidaridad y el desarrollo». En Comunicando para la solidaridad y la cooperación. Cómo salir de la encrucijada, editado por Javier Erro Sala y Teresa Burgui, 113-135. Pamplona: Foro de Comunicación, Educación y Ciudadanía.

Nos Aldás, Eloísa, Amador Iranzo y Alessandra Farné. 2012. «La eficacia cultural de la comunicación de las ONGD: los discursos de los movimientos sociales actuales como revisión». CIC. Cuadernos de Información y Comunicación 17: 209-237.

Rizzardini, Marco. 2002. "La producción de conocimiento y comunicación de las ONGD. Entre la ideología dominante y un imaginario social alternativo». En Las ONG y la política, editado por Marisa Revilla Blanco, 286-345. Madrid: Istmo.

Saiz Echezarreta, Vanesa. 2009. La solidaridad, espacio de mediación de los sentimientos morales. Análisis de la publicidad de las ONGD. Tesis doctoral. Madrid: Departamento de Periodismo III, Facultad de Ciencias de la Información. Universidad Complutense de Madrid.

Santolino, Montse. 2010. «Recuperando la esencia: Las ONGD como agentes de comunicación para el cambio social». En Comunicando para la Solidaridad y la Cooperación. Cómo salir de la encrucijada, editado por Javier Erro Sala y Teresa Burgui, 221256. Pamplona: Foro de Comunicación, Educación y Ciudadanía.

UNICEF. 2017. \#CierraUnicef. Acceso del 15 de diciembre de 2018 https://vimeo.com/207617226 



\title{
La escuela como factor de resiliencia
}

\author{
El caso de las y los jóvenes refugiados en Lyon (Francia)
}

Sandra Torres Acosta

sandra.to12@gmail.com 


\section{Resumen}

En la actualidad, asistimos a un aumento vertiginoso del número de personas forzadas a huir de su país de origen debido a conflictos, persecuciones y violencia generalizada. Según ACNUR, en el 2017 esta cifra ascendía a 68,5 millones, casi el doble de la registrada en 1997. De estas personas, 25,8 millones son refugiados y alrededor de la mitad tienen menos de 18 años. El contexto de guerra para esta población de niños y jóvenes ha significado la pérdida de sus seres queridos y cuidadores, de su hogar, de sus amigos y, en general, de su infancia. La apertura de heridas físicas y psicológicas puede comprometer de una manera determinante su visión de futuro, así como las posibilidades de retomar su vida normal. También puede afectar a su integración en el país de acogida, con otra cultura, idioma y costumbres diferentes.

Como ámbito de socialización, la escuela representa el primer dispositivo de integración de los niños y jóvenes refugiados en esta nueva cultura de acogida, así como la posibilidad de reconstruir su futuro. Frente a esto, cabe preguntarse lo siguiente: ¿puede la escuela asumir este reto y contribuir al cierre de estas heridas profundas causadas por la guerra, ofrecer la posibilidad de un mejor futuro y contribuir a reencauzar la vida de estas personas? En otras palabras, ¿̇la escuela puede llegar a asumir un rol de protección y ser un factor de resiliencia para esta población joven refugiada? $\mathrm{O}$ al contrario, ¿los niños, niñas y adolescentes refugiados deben ser resilientes para integrarse en la escuela?

Palabras clave: escuela, resiliencia, refugio.

\section{Introducción}

Nacer y crecer en un contexto de guerra y violencia, de huida y refugio, marca de manera definitiva la vida de una persona. Su impacto sobre la infancia constituye entonces un asunto primordial, puesto que puede llevar a la formación de un ser humano cargado de dolor, sufrimiento, odio o resentimiento, acrecentando sus vulnerabilidades y restringiendo sus posibilidades de desarrollo, nublando de este modo la posibilidad de concebir un proyecto de futuro y realización personal satisfactorios.

Desde el siglo $x x$, millones de niños $y$ jóvenes se han visto obligados a dejar su país de origen para huir de la guerra con sus familiares, o muchas veces sin ningún acompañante. Muchos de ellos han tenido que pasar por procesos de llegada traumáticos - los que han conseguido llegar-, exponiéndose a todo tipo de peligros y teniendo que sufrir tratos degradantes. Una vez en el país de acogida 
o reasentamiento, deben continuar con su vida en otro país, teniéndose que adaptar a un nuevo contexto con sus heridas todavía abiertas.

Después de la familia, la escuela representa la posibilidad de integración y desarrollo de un individuo. Por este motivo, la primera forma de que los pequeños refugiados comiencen una nueva vida es retomar sus estudios en el país de acogida. Sin embargo, llegarán a integrar los espacios educativos formales en unas condiciones no solo diferentes, sino también de desventaja frente a sus compañeros de clase. En este caso, la escuela como institución puede desempeñar un rol fundamental en la integración y la acogida exitosas de estos niños, convirtiéndose en un verdadero refugio, en la medida en que puede ofrecer un espacio saludable y de abrigo que los aleje del peso psicológico que llevan consigo y ayudarles a reconstruir su manera de ver la vida, en pocas palabras, puede convertirse en un espacio de resiliencia.

En ese sentido, mi pregunta será la siguiente: ¿la escuela ha sido realmente el lugar donde estos jóvenes han encontrado aquellos factores de protección o resiliencia que los han ayudado a continuar con su vida y a realizarse de manera satisfactoria en el país de acogida o, por el contrario, existen otros espacios o factores que han cumplido un rol fundamental en su proceso resiliente?

\section{Objetivos}

El objetivo general de esta investigación es evaluar el rol que ha desempeñado la escuela en cuanto al proceso resiliente de los jóvenes refugiados acogidos en Lyon (Francia). La importancia de dicha evaluación radica en que puede arrojar pistas importantes acerca de la manera en que se puede alentar a la población refugiada para que continúe con su vida de la mejor manera posible y se cierren las heridas que perpetúan historias de violencia y dolor. Pretende también mostrar las dificultades y tropiezos que los países de acogida deben enfrentar con el fin de tomar medidas para garantizar a estas personas un futuro más prometedor y viable, así como una futura historia más humana para todos los hombres y mujeres.

\section{Material y método}

Existen varias definiciones de resiliencia según el punto de vista adoptado por los investigadores. La resiliencia puede definirse como un proceso dinámico y complejo, resultado de una interacción entre el individuo y su contexto y por el cual, a pesar de su exposición a situaciones de adversidad o traumáticas, logra retomar su desarrollo normal y continuar su vida de manera satisfactoria (Cyrulnik 1998). 
Como proceso adaptativo y positivo en el marco de una situación de adversidad notable, la resiliencia puede variar en función del desarrollo del sujeto y de su entorno. Así pues, no es algo que el sujeto pueda adquirir de una vez por todas; es decir, un individuo no puede ser resiliente siempre frente a todas las adversidades que tengan lugar en su vida. Se habla, por lo tanto, de un proceso donde interactúan factores de protección (personales, familiares o ambientales), que contribuyen a forjar una personalidad resiliente.

Dentro de las diferentes concepciones sobre la resiliencia, hay consenso en cuanto a que existen factores protectores que influyen en el desarrollo y las trayectorias vitales de los sujetos que enfrentan adversidades y logran recuperarse de manera notoria, sin que se genere ningún tipo de disfunción social o psicológica. En general, estos factores de protección se clasifican en tres categorías: la primera corresponde a las disposiciones personales; como el temperamento, la capacidad de resolver problemas, la autonomía y la confianza en sí mismo. En segundo lugar, encontramos la cohesión y el entorno familiares; Por último, cabe mencionar el entorno social o externo a la familia, donde se puede ubicar a los amigos, parejas, comunidades identitarias y escuela. Todos estos factores pueden promover la adaptación positiva y evitar la generación de daños.

Sin embargo, a pesar de los esfuerzos por identificar los factores protectores más influyentes, apenas se ha intentado desarrollar investigaciones basadas en medidas propias del campo de estudio de la resiliencia. De hecho, la resiliencia comprende un conglomerado de factores de protección y niveles que se evalúan sobre la base de mediciones de otras áreas de investigación. Por lo tanto, la medida de la resiliencia puede tener una diversidad de contenidos $y$ elementos diseñados para evaluar y tener en cuenta características como la edad y el sexo.

Existen escalas de resiliencia centradas en diferentes factores, como, por ejemplo, rasgos de carácter, personalidad, apoyo social o calidad de las relaciones interpersonales. Estos son algunos de los más comunes cuando nos referimos concretamente a la resiliencia de los jóvenes o adultos.

La resiliencia en este trabajo se entenderá como un proceso que involucra una variedad de factores internos o externos, teniendo en cuenta características personales, relacionales, ambientales 0 familiares.

En nuestra hipótesis, se hizo referencia a factores centrados en las relaciones con el entorno escolar; es decir, centramos nuestra investigación en factores externos o ambientales en lugar de en factores internos o personales del individuo resiliente.

En este caso, se han utilizado dos métodos: el primero de ellos es la aplicación de una escala de resiliencia, instrumento mediante el 
cual se eligió a las personas que participarían del estudio, pues si se trata de una evaluación de los factores que contribuyeron a la resiliencia, es necesario contar con personas que sean resilientes. Existen muchas escalas, pero nos decantamos por la escala de EgoResiliencia, que es una de las escalas más simples porque tiene solo 14 ítems $^{1}$ y busca estudiar un conjunto de características estables de la personalidad susceptibles de adscribirse a una persona definida como resiliente (Anaut 2005, 46).

Como segundo método para complementar el estudio, se ha utilizado la entrevista semidirectiva. Se construyó una guía de entrevista que permitió dirigir las respuestas y el discurso de los jóvenes entrevistados para obtener información más precisa sobre su proceso resiliente. Al tratarse de una entrevista semidirectiva, algunas preguntas se agregaron o eliminaron según el caso. Además, no se grabaron todos los testimonios, pues algunas personas no querían ser grabadas o en ocasiones resultaba más cercano enfocarlo como un diálogo natural. En efecto, el tema del refugio se caracteriza por ser sumamente delicado, pues las personas que lo viven han estado expuestas a situaciones extremas donde su propia supervivencia se ha visto comprometida muchas veces, de modo que su seguridad y la confidencia de sus relatos de vida son prioritarias, así como la construcción de espacios de confianza.

Las organizaciones encargadas de apoyar a las personas refugiadas son muy reacias a facilitar o a permitir el acceso a la información necesaria para este tipo de estudios, razón por la cual la muestra con la que se contó para realizarlo fue de muy pocas personas. Se recogió un total de ocho testimonios en un transcurso de cuatro meses, por lo que los resultados no podrían extrapolarse como algo generalizable. Respecto al acceso a dichas personas, por lo general solo fue posible abordarlas a través de un intermediario muy cercano a ellas, que nos presentaba de manera personal, nunca por teléfono u otro medio no presencial. También es necesario aclarar que, en aras de garantizar la confidencialidad de estas personas, no se utilizaron sus nombres reales.

Aunque nuestra investigación se centra en los niños, niñas y jóvenes refugiados, entrevistamos a personas que ya habían finalizado la secundaria o incluso estaban iniciando sus estudios de grado, pero que llegaron siendo niños o niñas al país de acogida, en este caso Francia. De esta manera, tratamos de precisar con mayor claridad si realmente habían vivido un proceso resiliente, lo cual solo se puede determinar una vez transcurridos algunos años desde la situación adversa o traumática.

\footnotetext{
${ }^{1}$ "The Resilience Scale» fue creada por Wagnild y Young en 1993. Se puede consultar el original en inglés aquí: http://ja.cuyahogacounty.us/pdf_ja/en-us/defendingchildhood/drcharlesfigleyscoring-scalesheets.pdf
} 
Como se emplearon dos métodos para realizar la investigación, se presentarán en un primer momento los datos obtenidos del instrumento cuantitativo (es decir, de la escala de Ego-Resiliencia) para después describir los resultados hallados en los testimonios recogidos mediante las entrevistas semidirectivas.

En las siguientes gráficas se encuentran resumidos los datos recogidos en la aplicación de la escala de Ego-Resiliencia a la muestra seleccionada.

Tabla 1. Nivel de resiliencia de las personas participantes

\begin{tabular}{|c|c|}
\hline Personas refugiadas & Resultados \\
\hline Lydia & 80 \\
\hline May & 83 \\
\hline Tatiana & 77 \\
\hline Bahar & 73 \\
\hline Sean & 67 \\
\hline Ezéquiel & 72 \\
\hline Danilo & 74 \\
\hline Freddy & 76 \\
\hline
\end{tabular}

Nivel de resiliencia: 98-82: Tendencia muy alta de resiliencia; 81-64: Tendencia alta de resiliencia; 63-49: Tendencia media; 48-31: Tendencia baja de resiliencia; 30-14: Tendencia muy baja de resiliencia.

\section{Tendencia resiliencia}

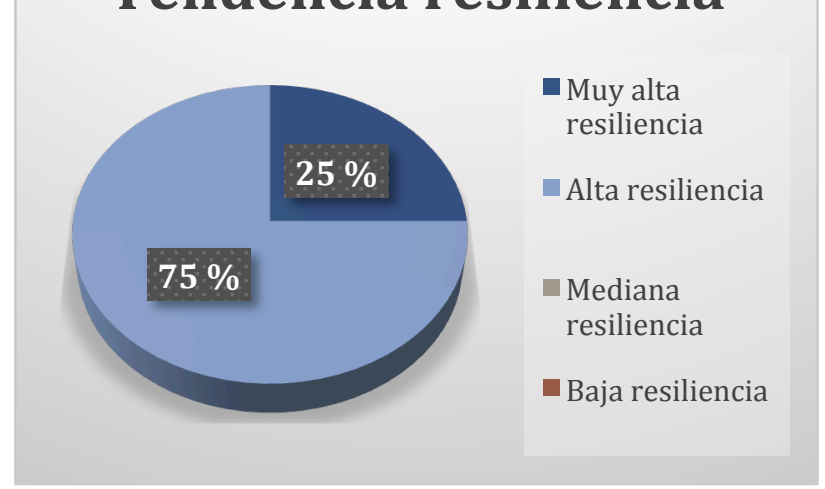

Gráfico 1. Tendencias hacia la resiliencia 
Como se ha sugerido en la selección de la muestra de la investigación, todos los refugiados que participaron presentan cierta tendencia hacia la resiliencia; de ellos, el $25 \%$ muestra una resiliencia muy alta y el $75 \%$ se inscribe dentro de una tendencia alta de resiliencia.

El promedio encontrado en el grupo es de 76,8; es decir, las personas que conforman la muestra de la investigación tienden a una resiliencia alta.

La escala de Ego-Resiliencia nos dio algunas pistas sobre las capacidades o factores internos propias del sujeto que desempeñan un papel importante en la construcción de esas personalidades resilientes. El objetivo de esta escala es medir dichas capacidades para adaptarse. Por lo tanto, analizaremos la respuesta dada a la muestra teniendo en cuenta los enunciados de cada categoría para profundizar los factores internos de resiliencia. Las categorías mencionadas son la flexibilidad, las habilidades sociales y la curiosidad.

En el siguiente gráfico se pueden apreciar las categorías más presentes en las personas que integraron la muestra:

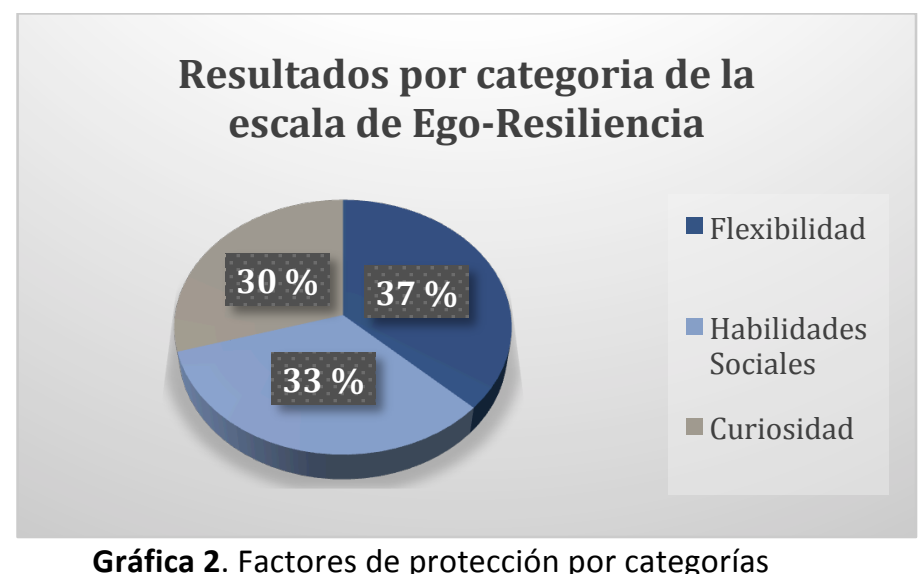

Según estos resultados, la característica más importante es la flexibilidad. En este sentido, podemos decir que nuestro grupo de refugiados resilientes tiene una gran capacidad para adaptarse a situaciones particulares manteniendo el control de eventos inesperados. De hecho, estas personas tuvieron que enfrentarse a un contexto completamente nuevo cuando llegaron a Francia.

Por otra parte, las entrevistas realizadas a las ocho personas refugiadas en Lyon (Francia) fueron transcritas o escritas directamente. Lo que se presentará a continuación son algunas de las frases que caracterizaron el relato de cada una de estas personas, así como algunos extractos que arrojaron pistas para analizar y dar respuesta a la pregunta de la investigación. 
Tabla 2. Frases representativas del grupo de personas participantes

\begin{tabular}{|c|c|}
\hline & Frases resilientes \\
\hline Lidya & $\begin{array}{l}\text { El reencuentro con mi familia fue lo más positivo y lo } \\
\text { que más me ayudó estando en Francia. }\end{array}$ \\
\hline May & $\begin{array}{l}\text { A pesar de mis dificultades con la lengua, yo he vivido } \\
\text { aquí momentos de tranquilidad. }\end{array}$ \\
\hline Tatiana & Yo veo siempre el lado positivo de la vida. \\
\hline Bahar & $\begin{array}{l}\text { El proceso de la vida es como las estaciones; algunas } \\
\text { veces hace mucho frío, pero después sabes que vendrá } \\
\text { el calor. }\end{array}$ \\
\hline Sean & $\begin{array}{l}\text { Yo creo que ahora tengo la fuerza para superar } \\
\text { cualquier cosa. }\end{array}$ \\
\hline Danilo & Tengo la capacidad de aceptar la realidad. \\
\hline Ezequiel & $\begin{array}{l}\text { En la vida, hay que estar siempre preparado para todo. } \\
\text { Uno no debe siempre ser demasiado optimista porque } \\
\text { después te sentirás decepcionado, ni tampoco muy } \\
\text { pesimista para conservar la esperanza y el gusto por la } \\
\text { vida. }\end{array}$ \\
\hline Freddy & $\begin{array}{l}\text { Yo vengo de un lugar donde todo el mundo se ayuda, } \\
\text { es por eso por lo que a mí me gusta ayudar a los } \\
\text { demás. }\end{array}$ \\
\hline
\end{tabular}

En los diferentes testimonios, se pudo constatar que el primer contacto de las personas entrevistadas con un entorno educativo fue recibiendo clases para aprender el idioma del país de acogida (en este caso, el francés) como requisito para su adaptación. Por tanto, cabe señalar que el acceso a la educación para estas personas, al menos en el campo del lenguaje, tiene un significado decisivo para una socialización favorable en la cultura de acogida y para continuar con sus estudios formales.

La mayoría de estas personas consideran la educación formal y el hecho de haber continuado con sus estudios dos pasos fundamentales que han favorecido su integración en Francia; es más, muchas de ellas se encuentran en el proceso de continuar con su grado o licenciatura. El éxito escolar que han conseguido las ha alentado a continuar, aunque para conseguirlo hayan tenido que sortear muchos obstáculos. Como dice Lydia: «al principio mis notas eran muy malas, pero fui progresando con el tiempo y ahora voy a presentarme al grado de Ciencias de la Educación, y me siento con la capacidad para hacerlo». En los casos de May y Bahar, por ejemplo, se encontraron muchas veces con evaluaciones denigrantes 0 
pesimistas, como cuando un profesor le dijo a Bahar que a él no lo

habían formado para escribir y que, por tanto, nunca lo haría bien; o cuando May preguntaba a sus compañeros de clase sobre algo y le respondían que buscara en Internet. Según sus relatos, los profesores no tenían ningún interés en saber de dónde venían o en conocer su cultura, por lo que la relación era muy distante. En cuanto a sus compañeros o pares, la percepción es que eran muy competitivos e individualistas.

En cuanto a Ezéquiel, Freddy, Sean y Danilo no hacen ninguna referencia a su vida escolar o a alguien de la escuela cuando hablan de aquello que más les ha ayudado o motivado a continuar durante su estancia en el país de acogida. Sin embargo, en sus testimonios resaltan la motivación y el ánimo que les transmitieron algunas personas voluntarias en el centro de aprendizaje de francés, cuando acababan de llegar al país. Ezéquiel, por ejemplo, habla de su profesora de francés, quien lo motivó a aprender la nueva lengua y a ayudar a otros a través de lo que él sabía hacer: cortar el pelo. Así, pudo volver a sentirse útil, ayudar y aportar a otras personas en la misma fundación donde le tendieron la mano a él.

La familia ha desempeñado un papel determinante. Así, la madre de Lydia y Tatiana, así como el tío de May, representan el apoyo para superar la adversidad y darles las fuerzas necesarias tanto para continuar luchando por mejorar su condición como para adaptarse social y culturalmente a la sociedad de acogida.

En el caso de Sean y Freddy, más que su familia han sido los grupos comunitarios a los que han pertenecido, específicamente grupos religiosos, donde han encontrado nuevas esperanzas, ideales y personas que se han convertido en su nueva familia.

Por último, el caso de Danilo es más complicado, puesto que aún no se le ha concedido el asilo, lo que ha provocado que no tenga acceso a muchos servicios en el país de acogida. Sin embargo, a pesar de enfrentarse a más obstáculos, ha logrado mantener el gusto por la vida y seguir luchando por estar mejor. En consecuencia, podríamos referirnos a que es más su fortaleza personal y la confianza en sí mismo lo que lo ha ayudado.

\section{Discusión y conclusiones}

Teniendo en cuenta los resultados del estudio, se podría decir que la escuela no ha sido un lugar que les haya ayudado en su proceso de resiliencia a las personas participantes. Si bien tiene un papel importante en cuanto a su integración y puede garantizar un futuro con más posibilidades, al mismo tiempo implica presiones 0 situaciones que pueden ser desmotivantes y ponen en riesgo el proceso resiliente. Lo anterior se puede constatar en el modo de interacción distante que se da entre los profesores y los estudiantes, 
o bien entre los propios estudiantes, así como en la tendencia a la homogenización de todas las personas en la escuela, sin importar su diversidad social y cultural. Las dinámicas de la escuela, por tanto, no son integradoras, sino que exigen que sean las personas las que se adapten a la escuela y no al contrario. También cabe resaltar el carácter competitivo de la escuela. Las relaciones que se establecen no están basadas en la solidaridad, sino en quién es mejor o peor. Dadas las condiciones de desventaja de las que parten los niños y niñas refugiados, puesto que vienen de otros sistemas educativos, con otros métodos y dinámicas, sumado a la repercusión que la salida abrupta de su país ha causado en ellos, esta concepción de la educación no favorece en absoluto su proceso resiliente. Al contrario, los hace ponerse constantemente en comparación los demás, que a su vez los ven como un competidor más. Las formas de evaluación resultan asimismo dispositivos que fomentan esta forma de concebir el aprendizaje, quién sabe más y quién menos, al considerar los errores como fracasos y no como oportunidades para mejorar. Así pues, para las personas de la muestra, la escuela no fue un lugar acogedor o favorecedor de la resiliencia, sino que percibieron que hay que ser resiliente para estar en la escuela.

Por otra parte, los cursos de idiomas para las personas que llegan por primera vez a Francia son, en su mayoría, desarrollados por asociaciones. Estas asociaciones establecen diversas formas de apoyo a través de voluntarios que, a su vez, son más que simples transmisores de conocimiento. El voluntariado, en la mayoría de los casos, significa que estas personas toman su trabajo con una disposición diferente porque se comprometen libremente. Este compromiso hace que su trabajo sea más autónomo y satisfactorio, más allá de las presiones académicas, la evaluación, la competencia $y$, en general, los propios requisitos y dinámicas de la escuela, lo que conduce a forjar relaciones más cercanas y empáticas con los estudiantes, siendo un factor para que muchos encuentren más apoyo y motivación en estos espacios y con estas personas que en la escuela.

Se podría afirmar, según los resultados, que en el grupo de nuestra muestra, existe una combinación de factores de protección externos e individuales que han sido claves en su proceso resiliente, pero estos factores hacen parte en su mayoría de su ámbito familiar o social. En ese sentido, el factor familiar actúa como una especie de escudo al neutralizar el impacto de los factores de riesgo a los que están expuestas las personas refugiadas. Es importante tener en cuenta que los puntajes más altos en la escala de resiliencia corresponden a personas que han establecido un fuerte vínculo con su familia.

Los grupos religiosos, por su parte, funcionan como una red social de apoyo que reconstruye las bases de confianza y solidaridad, 
junto con el sentimiento de pertenencia social, una de las pérdidas

que han sufrido estas personas al tener que dejar su país y llegar a otro del cual no se sienten parte.

Por último, encontramos los factores individuales o propios de cada de una de estas personas. Como se había afirmado anteriormente, al entenderse la resiliencia como un proceso, es muy probable que los factores de protección internos se hayan desarrollado desde una edad muy temprana, pudiendo ser el resultado de otros factores de protección como un entorno familiar muy favorable o la creación de vínculos realmente significativos y positivos tanto con sus pares como en su comunidad.

La escala de Ego-Resiliencia, que medía la capacidad de adaptación de los sujetos después de eventos traumáticos o adversos a través de tres capacidades (la flexibilidad, la curiosidad y las habilidades sociales), ha demostrado que las personas que participaron en el estudio tienen una mayor tendencia a la flexibilidad. En efecto, estas personas tuvieron que afrontar un contexto completamente nuevo al llegar a Francia: encontrar medios para expresarse y comunicarse a pesar de no saber el idioma, dormir en la calle, conocer el funcionamiento burocrático y legal del nuevo país para regular su estancia, desenvolverse en un ambiente social con otros códigos y formas de relacionarse. Todos estos aspectos conducen a una adaptación radical que ni siquiera esperaban, pues muchos ni siquiera tuvieron la oportunidad de escoger a dónde ir.

Esta capacidad de adaptación puede verse influida por una percepción de la efectividad desarrollada por estas personas. De hecho, este sentimiento influye en sus formas de resolver problemas y les permite el manejo del estrés en situaciones difíciles. Por lo tanto, está íntimamente vinculado a la percepción que tiene el individuo de su propia capacidad para modificar y actuar en los eventos que ocurren en su vida. Entonces, podemos decir que la alta flexibilidad de estas personas también puede asociarse con un fuerte sentimiento de autoeficacia.

Las habilidades sociales son la segunda categoría más prominente. De hecho, las habilidades sociales demuestran, en primer lugar, un desarrollo social normal y también son importantes para cualquier individuo resiliente, pues las redes sociales son un factor externo de resistencia para fortalecer la autoestima del individuo y la percepción de su propio valor. La autoestima se desarrolla en la interacción con los demás, es decir, es el resultado de la autoevaluación desde los otros. Podemos afirmar entonces que los individuos de nuestra muestra tienen la oportunidad de desarrollar una percepción favorable de sí mismos a través de sus habilidades sociales.

Finalmente, la curiosidad se refiere a la posibilidad de que las personas construyan metas en sus vidas y planifiquen para el futuro. 
De hecho, la curiosidad despierta el deseo de descubrir y aprender, así como el interés en la vida. Sin embargo, en los resultados la curiosidad es la categoría más baja en el grupo de refugiados. Esto puede explicarse por el hecho de que la mayoría de estas personas pensaron en sus vidas en el hogar, por lo que sus intereses y planes de vida estaban vinculados a su país. La repentina llegada a Francia los obligó a cambiar sus planes y adaptarlos al nuevo país. Están, entonces, en una situación en la cual reconstruir su presente es decisivo para pensar en el futuro.

En definitiva, al ver las conclusiones del estudio, es necesario comenzar a evaluar la educación y la escuela, así como reconsiderar sus dinámicas, estructura, roles y objetivos, respecto a la acogida de personas que provienen de otras culturas y contextos adversos, como es el caso del refugio. La escuela no puede seguir siendo un lugar estático, al que las personas tengan que adaptarse, sino al contrario, que sea el centro escolar el que tenga la posibilidad de adaptarse a las personas, para aprovechar al máximo su potencial en cuanto a la formación de seres con un sentido cada vez más humano e integrador.

\section{Bibliografía}

ACNUR. 2000. La situación de los refugiados en el mundo 2000. Cincuenta años de acción humanitaria. Barcelona: Icaria Editorial.

ACNUR. 2010. Asylum levels and trends in industrialized countries. Acceso en https://www.unhcr.org/statistics/unhcrstats/4d8c5b109/asylum -levels-trends-industrialized-countries-2010-statisticaloverview.html

Anaut, Marie. 2008. La resiliencia: superar los traumatismos. Barcelona: Gedisa Editorial.

Anaut, Marie. 2015. Psychologie de la résilience. París: Armand Colin.

Cyrulnik, Boris. 1999. Un merveilleux malheur. París: Odile Jacob.

Cyrulnik, Boris y Jean Pierre Pourtois. 2007. École et résilience. París: Odile Jacob.

Global Migration Data Analysis Centre (GMDAC). 2018. Global Migration Indicators. Acceso en https://gmdac.iom.int/globalmigration-indicators-2018-report.

Machel, Graça. 1996. Impact of armed conflict on children. Nueva York: Naciones Unidas. 
Masten, Ann S. 2011. "Resilience in children threatened by extreme adversity: Frameworks for research, practice, and translational synergy». Development and Psychopathology 23 (2): 493-506.

Wagnild, Gail M. y Heather M. Young, 1993. «Development and psychometric evaluation of the Resilience Scale». Journal of Nursing Measurement 1 (2): 165-178.

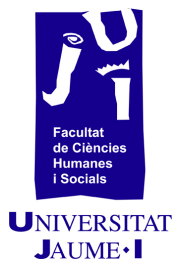





\section{La ética del discurso en la filosofía con niñas y niños}

Anca-Nicoleta Rotila

al285623@uji.es 
I. Resumen

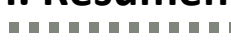

En este estudio vamos a intentar determinar en qué medida la filosofía con niñas y niños sigue las mismas normas que se determinan en la ética del discurso, así como decidir si dicho método filosófico cuenta con una eficacia cultural. Para responder a nuestra pregunta nos hemos centrado en el análisis de investigaciones de las autoras Haynes, Nos, Irando y Ferné, entre otras no menos importantes. Así, a lo largo del texto se podrá ver claramente la estrecha relación entre la filosofía con niñas y niños y la ética del discurso, que se muestra en la importancia del diálogo, de la búsqueda de una comunidad comunicativa ideal y el no perseguir objetivos individuales o estratégicos. De igual manera, la filosofía con niñas y niños cuenta con la eficacia cultural, ya que empuja a los más pequeños y a las más pequeñas hacia el pensamiento reflexivo sobre el bien común.

Palabras clave: filosofía, niñas, niños, ética, discurso, eficacia cultural.

\section{Introducción}

La filosofía con niñas y niños considera y apoya que las y los infantes tienen sentido crítico, contrariamente a lo que desde Occidente se ha inculcado. De esta manera, en estas clases de filosofía se sigue la metodología del diálogo que, como sabemos, es la base de la ética del discurso. No obstante, la filosofía con niñas y niños también se presenta como una reflexión activa, por lo tanto analizaremos su eficacia cultural.

Dicho esto, el objetivo que se persigue en este escrito es determinar en qué medida la filosofía con niñas y niños sigue los mismos pasos que la ética del discurso y averiguar si esta filosofía tiene, o consigue, una eficacia cultural.

A lo largo del texto hablaremos de la fundamentación del tema, es decir, de aquello que ya se ha escrito. En el siguiente paso, enumeraremos la metodología que utilizaremos para el análisis de nuestro tema. A continuación, detallaremos los resultados obtenidos del análisis de los textos utilizados. Seguidamente, en la discusión, analizaremos, por una parte, si la filosofía con niñas y niños sigue las mismas pautas de la ética del discurso en sus clases. Además, vamos a hablar sobre la eficacia cultural de esta filosofía. Por último, haremos una pequeña reflexión a modo de conclusión. 


\section{Fundamentación}

Aunque la asociación entre la filosofía con niñas y niños y la ética del discurso no se ha hecho, la ética del discurso sí que ha estado analizada en varias ocasiones por su influencia en otras temáticas. Una de estas es la ética de la liberación sobre la que Enrique Dussel ha escrito en su artículo "La ética de la liberación ante la ética del discurso». En este texto, el autor se basa en el trabajo de Karl-Otto Apel "La ética del discurso ante el desafía de la filosofía de la liberación» para analizar la objeciones que este hace. Con otras palabras, estas dos disciplinas son puestas en discusión para averiguar cómo se influyen una a la otra (Dussel, 1996).

Otro texto en el que se compara un tema con la ética del discurso es el artículo de Juan Carlos Siurana "La ética del deporte desde la justicia como igualdad de oportunidades en la ética del discurso». En este texto se explica la importancia de la ética del deporte para que, ulteriormente, se hable sobre la influencia de la ética del discurso en el deporte, considerando la justicia como igualdad de oportunidades (Siurana, 2007).

En cuanto a la eficacia cultural, según la investigación sobre los escritos publicados en relación con este tema, solo se han encontrado textos que hablen de la eficacia cultural en el medio de la comunicación. Sin embargo, en este trabajo hablamos de la eficacia cultural de una disciplina educativa como es la filosofía con niñas y niños.

\section{Metodología}

Como hemos dicho anteriormente, el objeto de estudio es, por una parte, ver en qué medida la filosofía con niñas y niños sigue las mismas pautas que la ética del discurso. Para ello, lo primero que se ha hecho ha sido analizar textos en los que se explique el objetivo de la filosofía con niñas y niños y su desarrollo en clase, o la aplicación que tiene. Uno de los textos revisados ha sido el libro de Johanna Haynes Los niños como filósofos. El aprendizaje mediante la indagación y el diálogo en la escuela primaria. El segundo escrito fue el artículo de Guillermina Ferrer Rodríguez «Filosofía con niños. Como acercar la filosofía a niñas y niños».

Por otra parte, para el análisis de la ética del discurso, se ha revistado un apartado de la tesis de Sonia París Albert "La transformación de los conflictos desde la Filosofía para la Paz». En este capítulo de la tesis, la autora explica la ética del discurso para aplicarla a la resolución de los conflictos. En este texto se han buscado cuáles son los pilares básicos sobre los que se sustenta esta ética. 
Por último, para hablar sobre la eficacia cultural me he basado

en el artículo de las autoras Eloísa Nos, Amador Irando y Alessandra Ferné «La eficacia cultural de la comunicación de las ONGD: los discursos de los movimientos sociales actuales como revisión». En este texto se explica muy bien qué es la eficacia cultural para poder aplicarla, también, a otras disciplinas o temáticas.

Después de la lectura de los textos, se han extraído los resultados deseados y, ulteriormente, las tres temáticas analizadas puedan ser puestas en discusión una con la otra.

\section{Resultados}

\subsection{La filosofía con niñas y niños}

Como se ha dicho en el apartado anterior, en el análisis del libro de Johanna Haynes y del artículo de Guillermina Ferrer Rodríguez, se ha buscado información sobre qué es la filosofía con niñas y niños y los objetivos que persigue, el desarrollo de estas clases y las pautas comunicativas que se emplean en ellas. Comentamos todas estas cuestiones a continuación:

Actualmente, la filosofía se ve como un estudio meramente académico y elitista, a cuya terminología compleja pocos pueden acceder. De esta manera, la filosofía se aprende en las aulas de memoria pero en muy pocos casos se aprende realmente a filosofar. Por eso, en 1970 se ponen las bases del proyecto «Filosofía con niños", que lleva a cabo el profesor y autor Lipman. El propósito de este proyecto fue "ayudar al estudiantado al desarrollo de una actitud crítica y la necesidad de participación activa» (Ferrer Rodríguez, 2012: 23). En cuanto a la metodología, esta tiene como pilares básicos el diálogo y la creatividad. Es más, la filosofía con niñas y niños apoya el artículo 12 de la Convención de las Naciones Unidas sobre los Derechos del Niño por el que se explica que se debe dejar al niño y a la niña a que expresen sus opiniones libremente y a que formen sus propias maneras de pensar, su proprio juicio; con otras palabras: que se tengan en cuenta sus opiniones, claramente, en función de su edad y madurez. Esto les permitirá participar de forma activa en sus comunidades y naciones (Haynes, 2004: 35).

No obstante, como bien sabemos, en torno al estímulo del pensamiento crítico, hoy en día solo se escucha hablar en las aulas de cursos más avanzados, como las de bachillerato o -y sobre todoen la universidad. Sin embargo, el pensamiento crítico debe ser una cualidad que se ha de trabajar desde una edad temprana. Contrariamente a lo que muchos piensan, los niños y las niñas nacen con capacidad de crítica, lo que significa que el pensamiento adulto, occidental, se equivoca al decir que los adultos son los que les deben transmitir los conocimientos, la manera de pensar o las ideas. Esta 
es, sin duda, un fomento de la educación bancaria de la que Paulo

Freire tanto nos hablaba. Sin embargo, la pregunta surge en torno a si verdaderamente tanto los padres como los profesores y las profesoras siguen este patrón a la hora de interaccionar con los infantes.

Al mismo tiempo, la autora Ferrer Rodríguez nos habla del significado que tiene la filosofía para Lipman, como «una actividad reflexiva que se realiza tanto individual como conjuntamente» (Ferrer Rodríguez, 2012: 23). Con esto entendemos que se parte desde lo personal, la búsqueda de respuestas a las preguntas personales, siempre acompañado por los profesores y las profesoras, así como, por los compañeros y las compañeras. Al hablar de una clase, nos referimos a un espacio en el que convive una multitud de culturas, etnias y religiones, lo que ofrece diversidad de percepciones del mundo y maneras de argumentar. No obstante, la diversidad de temáticas abordadas tendrá como resultado contradicciones, pero también reconciliaciones (Ferrer Rodríguez, 2012: 24-25).

Johanna Haynes también respalda este argumento al decir que en clase, a la hora de trabajar un tema concreto -como hablar sobre la muerte- es importante empezar por un cuento que ayude a los niños y a las niñas a empatizar con el personaje y a conectar con sus vivencias personales (Haynes, 2004: 25-26). El método socio-afectivo, según mi punto de vista, hace que los niños puedan entrar mejor en la historia. Y lo mismo ocurro con preguntarles lo que les haga pensar en qué harían ellos, que deberían hacer los personajes, etc. Es más, es importante animarlos a que hagan ellos las preguntas.

A medida que van adquiriendo más seguridad en sí mismos, los niños dejan de plantear preguntas únicamente al adulto. Valoran cada vez más la oportunidad de discutir entre ellos los asuntos importantes. Cada vez se preocupan más de plantear cuestiones que tengan algo que ver con sus propias vidas. (Haynes, 2004: 29)

Así pues, el planteamiento de la filosofía con niñas y niños empieza necesariamente con cuestiones que ellos plantean, a partir de un estímulo previo, como una historia, un cuento, una imagen, etc. Al plantearse todas las preguntas, el debate se focalizará en aquellas escogidas de acuerdo a un proceso democrático. Se persigue que los niños y las niñas piensen de una manera lógica y crítica, pero sobre todo $-y$ es algo que ellos y ellas lo saben hacer perfectamente pero que con el paso del tiempo se pierde-, a pensar de una manera creativa. El objetivo final que se persigue no es que los infantes colaboren para llegar a una conclusión que obtenga unanimidad de apoyos a favor, sino que colaboren para llegar a la verdad a través de la diversidad de opiniones. Todo esto ayuda al desarrollo de la autoconciencia y la flexibilidad personal, entre otras muchas más aportaciones (Haynes, 2004: 33). Esta filosofía se 
plantea como una actividad en la que la clase se establece en círculo, de manera que todos puedan verse las caras y estar comunicados.

...se pretende conseguir una comunicación eficaz y buenas relaciones en el aula, así como ofrecer un foro en el que se puedan abordar los problemas con talante constructivo. Se anima a los niños a que exploren sus sentimientos, a que escuchen a sus compañeros, a que hablen por turno y a que busquen soluciones (Haynes, 2004: 32)

No obstante, lo que sí que tiene que quedar claro es que un debate filosófico con niñas y niños sigue los mismos requisitos que cualquier otro debate imparcial, en el que se pide la escucha atenta, la no interrupción de los discursos de los demás, ni la imposición de ideas o cualquier forma de ridiculización o falta al respeto de cualquiera de los miembros del grupo. En cuanto al profesorado, este debe escuchar a los niños y a las niñas, pero al mismo tiempo, encontrar formas originales y creativas para lograr que el alumnado también le escuche y que, ulteriormente, se pase a la acción (Haynes, 2004: 93). No hay que olvidar que hoy en día estamos intentando educar niños que pertenecen a una sociedad movida por las innovaciones tecnológicas mediante métodos tradicionales de enseñanza, lo que se debe revisar y cambiar.

Para concluir este apartado cabe decir que entre los objetivos de la filosofía con niñas y niños se puede encontrar que se promueve el razonamiento reflexivo y el pensamiento más allá de lo individual abarcando el bien común (Ferrer Rodríguez, 2012: 25). Además, encontramos que genera el juicio crítico entre los jóvenes, pero, sobre todo, les impulsa hacia pensar por sí mismos. También, les ayuda a hacer uso de la reflexión para encontrar sentido a sus experiencias de vida. Por último, fomenta la comunicación, un aspecto fundamental entre los jóvenes (Ferrer Rodríguez, 2012: 29).

\subsection{La ética del discurso}

Como se ha mencionado anteriormente, a continuación se enumerarán los resultados sobre el análisis del texto de la autora Sonia París Albert. No obstante, me gustaría empezar este apartado con la definición de diálogo que el autor Vicent Martínez Guzmán (2004: 302) nos ofrece:

Acción de comunicarse dos o más personas pidiéndose o dándose razones de por qué se hacen, dicen o callan, lo que se hacen dicen o callan. Etimológicamente proviene del griego diálogos que, a su vez, viene del verbo dialégomai que significa hablar, decir, conversar. Es una palabra compuesta de la preposición dia como prefijo que significa "a través de» y de logos que significa, palabra, razón, discurso [...] Así «diálogo» es algo que nos hacemos dos o 
más personas, a través de las palabras, de las razones que nos

damos, de los discursos e, incluimos, de los silencios comunicativos.

Según las palabras del autor podemos ver que, en la ética del discurso, cada uno tiene la responsabilidad sobre todo aquello que dice, hace o calla. También, el otro como receptor, debe mostrar atención y disposición para comprender aquello que se le comunica a través de gestos, palabras y silencios.

Partimos de la premisa que dos personas, cuando empiezan un diálogo, establecen su relación, la definen. Teniendo en cuenta que toda la vida de las personas se define a través de sus relaciones, podemos decir que no se puede no comunicar. "Así, observamos la comunicación como un acto inherente a las relaciones humanas, parte de nuestra conducta y que tiene diferentes consecuencias en función de cómo sea utilizado» (París Albert, 2005: 177). Decimos pues, que la comunicación tiene entre sus premisas la empatía, el reconocimiento, el entendimiento mutuo y la búsqueda de la verdad.

Así pues, a continuación describimos los puntos más importantes a seguir -según la ética del discurso- para que se llegue a una buena y mejor comunicación. El primer punto $y$, sin duda, uno de los más importantes, es la escucha activa. Esto implica un esfuerzo por parte del receptor para entender el contexto de aquello de lo que se habla y las emociones y percepciones del emisor. El segundo es que uno de los propósitos más importantes del emisor sea hacerse entender, ya que muchas veces, por falta de compromiso con aquello que queremos comunicar o por falta de paciencia con la capacidad de comprensión del receptor, no prestamos suficiente atención a un objetivo tan importante como es el de hacernos entender. Otro caso que se suele dar es que los actores de la acción comunicativa presten mayor atención al hecho de afirmar su posición en la discusión que a la comprensión recíproca. Asimismo, es importante hablar desde nuestro punto de vista, sobre nuestros propios pensamientos y creencias, y no opinar sobre el otro, sobre sus actuaciones o convicciones. No obstante, tener un objetivo claro, preestablecido, de aquello que quieres decir tiene mucha importancia. Eso ayuda a no perder el hilo conductor de la conversación, así como, a poder seguir las ideas que queremos exponer y a que nuestro receptor nos pueda entender con más facilidad. Por último, uno de los puntos que personalmente considero de mayor relevancia es «...hacer ajustes que tengan en cuenta las diferencias de personalidad, sexo y cultura» (París Albert, 2005: 178).

Sin embargo, aparte de aquello que nos decimos a través de la comunicación oral, de las palabras, también hay que prestar atención a la comunicación no verbal como los silencios o la gesticulación. Estos dos recursos juegan un papel significativo, y más aún cuando el 
silencio, según en qué circunstancias se utilice, puede tener unas consecuencias más o menos positivas. Esta idea del silencio se puede relacionar muy bien con la idea de interpelarnos unos a otros. Y la capacidad de interpelarnos significa que podemos pedirnos cuentas o pedirnos explicaciones por aquello que nos decimos, hacemos o callamos, en este caso. La interpelación en el silencio tiene que ver con la responsabilidad que asumimos a la hora de callarnos cosas. "Cualquier cosa que hacemos, decimos o callamos nos compromete, nos liga con los y las otras interlocutoras de tal forma que siempre podemos pedirnos cuentas y exigirnos nuestra responsabilidad» (París Albert, 2005: 180).

Siguiendo la misma línea, hay que decir que, en la ética del discurso, los mismos participantes son los que deben crear las normas que se han de cumplir para la buena comunicación y el buen desarrollo del discurso argumentativo. Esto garantiza la adecuación situacional de los afectados. Basándose en el consenso y el reconocimiento mutuo, las normas deben ser aceptadas por todos y cada uno de los afectados (París Albert, 2005: 184). No hay que olvidar que se busca una comunidad comunicativa ideal, pero, al mismo tiempo, una comunidad comunicativa real.

Avanzando en la idea, según la teoría de los actos de habla de Asutin y Searle, decir algo es realizar una acción. Por esto, entendemos que el acto de habla es una acción que tiene como finalidad el entendimiento. Sin embargo, para que el entendimiento entre todos los afectados se dé, se han de cumplir las pretensiones de validez del habla, que empiezan con el hecho de que el receptor entiende que el mensaje del emisor es verdadero, el mensaje es inteligible, la forma de realizar el mensaje es correcta y también que el receptor crea en la veracidad de la intención del emisor (París Albert, 2005: 185).

Otro punto relevante es el principio de universalización dentro de la ética del discurso. Según este, ninguna de las normas de las que hablamos anteriormente, se tomará como máxima, sino que se universalizará o ajustará según las circunstancias de afectado. Así pues, en el acto de la comunicación se aceptarán personas que saben que es el bien, que saben que es la justicia y que «...se entienden a sí mismas como la fuente de pretensiones legítimas y aceptan las condiciones de la cooperación equitativa» (París Albert, 2005: 186). Solo pueden entrar en la argumentación aquellos que garantizan la igualdad de derechos entre los participantes y la veracidad de sus aportaciones. Para concluir con esta idea, gracias al principio de universalización, solo se aceptaran las normas que benefician por igual a todos los afectados, y todo esto siguiendo el objetivo del reconocimiento recíproco (París Albert, 2005: 186).

No obstante, si definimos la acción comunicativa, esta tiene como pilar base el entendimiento lingüístico. Así, los afectados se 
dedicarán a cooperar, compartiendo un momento de la vida y basándose en las interpretaciones comunes que hacen sobre diferentes situaciones. Esto quiere decir que los participantes en el acto comunicativo no seguirán las pautas de la acción estratégica en la que ellos buscan su propio éxito, sino que seguirán las pautas de la acción comunicativa cuya finalidad es entenderse mutuamente. En la comunidad ideal de comunicación de la que se nos habla en la ética del discurso, lo que se persigue es el consenso entre los participantes (París Albert, 2005: 188).

En este tipo de ética, tanto en el mundo subjetivo del participante o hablante como en el mundo social, la veracidad es muy importante. Por ello, se pueden criticar los actos de habla si se considera que el enunciado no es verdadero, si se duda respecto a si la intención del hablante es veraz, o se puede considerar un acto de habla no correcto si no cumple con las normas preestablecidas y aceptadas por todos los participantes. Otro punto importante de la ética del discurso es la responsabilidad, en tanto que todos los participantes deben asumir la responsabilidad de todo lo que dicen y las consecuencias que surgen de ello (París Albert, 2005: 189-190).

En definitiva, la ética del discurso, que también se conoce como la ética de la comunicación, tiene como base el discurso argumentativo (París Albert, 2005: 183).

\subsection{La eficacia cultural}

Siguiendo lo dicho en el apartado de la metodología, a continuación redactaremos los resultados del análisis del texto de los autores Eloísa Nos, Amador Iranzo y Alessandra Ferné, centrándonos en los significados de la eficacia cultural.

El estudio de la eficacia cultural se aplica y sustenta sobre una base muy importante que es la performatividad de los discursos, que se refiere, en pocas palabras, a las competencias que algunas palabras o expresiones tienen de convertirse en acciones que, finalmente, transforman la realidad. Este concepto de eficacia cultural tiene implícita «la capacidad de influencia sobre la opinión pública» (Nos, Iranzo y Ferné, 2012: 215).

Sin embargo, un primer paso hacia la eficacia cultural lo tiene la indignación, es decir, una emoción moral activadora, de responsabilidad, que empuja a la participación, a la investigación sobre aquello que indigna, y al empoderamiento. «En otras palabras, la eficacia comunicativa se entiende aquí como la capacidad de activar la sensibilidad moral de la ciudadanía (por medio de la indignación) como camino hacia una sabiduría cultural» (Nos, Iranzo y Ferné, 2005: 216). 
A lo largo de los resultados hemos podido ver que gran parte de los pilares sobre los que se basa la ética del discurso se encuentran en la filosofía con niñas y niños. Nada más empezar con el análisis de la vinculación entre las dos, nos podemos dar cuenta de que, de estas, el diálogo es la acción más presente e importante. Como bien se sabe, por una parte, la ética del discurso se desarrolla en torno al diálogo y, por la otra, la filosofía con niñas y niños utiliza el diálogo como metodología de trabajo.

Siguiendo con el análisis, hemos dicho que en la ética del discurso se busca una comunidad comunicativa ideal, una comunidad comunicativa en la que todos tengan voz, en la que todos puedan dar su opinión y esta sea absolutamente válida, independientemente de la persona. En las clases de filosofía con niñas y niños también se busca que ellos y ellas tengan voz, que den su opinión y juntos encontrar soluciones sobre diferentes situaciones de la vida, ya que, contrariamente a lo que desde occidente se ha intentado inducir, los niños y las niñas tienen capacidad crítica. Así pues, podemos decir que tanto en este tipo de ética como en esta metodología filosófica, se busca llegar a una comunidad comunicativa ideal.

En estas comunidades comunicativas, la acción comunicativa que se lleva a cabo parte de una situación planteada previamente, son interpretaciones comunes sobre diferentes situaciones. Está claro que en el caso de la filosofía con niñas y niños, se parte de un estímulo previo, una historia contada previamente cuya plasmación ayude a que los infantes tengan más empatía con los personajes. No obstante, si hay algo que une a la ética del discurso y a la filosofía con niñas y niños es el objetivo. Y este, o la finalidad del diálogo, no es que se llegue a una conclusión apoyada por todos los participantes, sino que se llegue a la verdad a través de la diversidad de opiniones. Esto quiere decir que los participantes no perseguirán su propio éxito, sino que tendrán como propósito final el entendimiento mutuo. Así pues, la comunicación persigue la empatía, el reconocimiento, el entendimiento mutuo y la búsqueda de la verdad.

Sin embargo, si hay algo que define y une estos dos modelos teóricos es que siguen las mismas pautas de cualquier debate imparcial en el que se pide que se escuche atentamente, que no se interrumpan las intervenciones de los compañeros, que no se impongan las ideas; se rechaza cualquier forma de ridiculización o falta al respeto de cualquiera de los participantes a la discusión. No hay que olvidar que, niños, niñas o adultos, todos tenemos responsabilidad sobre aquello que decimos, hacemos o callamos, puesto que tenemos la posibilidad de interpelarnos, es decir, podemos pedirnos cuentas por aquello que nos decimos, hacemos o 
callamos. Y esto, porque, como decíamos anteriormente, la acción comunicativa o el acto de habla busca el entendimiento; sin embargo, para que este se dé, se deben cumplir las pretensiones de validez del habla, es decir, la verdad, la inteligibilidad, la rectitud normativa y la veracidad.

Asimismo, hay que considerar que, tanto en la ética del discurso como en la filosofía con niñas y niños, se tienen en cuenta las diferencias de sexo, cultura, personalidad, religión, etc. De esta manera, por una parte, en la ética del discurso, como se ha mencionado anteriormente, las normas creadas por todos los participantes antes de la comunicación siguen el principio de universalización, según el cual estas se deben ajustar a las circunstancias de cada uno. Por otra parte, en la filosofía con niñas y niños se aprovecha esta diversidad de culturas, etnias, religiones, como una diversidad de percepciones sobre el mundo y una multitud de maneras de argumentar.

A continuación, como se ha mencionado en la introducción de este trabajo, me gustaría hablar sobre la eficacia cultural de la filosofía con niñas y niños a través de los resultados del análisis de los textos.

Si miramos los resultados extraídos sobre la filosofía con niñas y niños, nos damos cuenta de que sí que persigue una finalidad activa, de transformación de la realidad. Aunque estemos hablando de niñas y niños, esta filosofía aboga por un empoderamiento de estos infantes que, mediante las reflexiones filosóficas, puedan llegar a participar de forma activa en sus comunidades y naciones.

Sin embargo, como hemos mencionado anteriormente, el primer paso hacia la eficacia cultural es la indignación. En este sentido, se persigue que el alumnado de la filosofía sobre la que estamos hablando piense de una manera lógica, crítica y creativa. Por tanto, vemos que la creatividad está presente en la filosofía con niñas y niños, lo que podemos relacionar con otro pilar básico de la eficacia cultural: la participación. Los profesores y las profesoras, aparte de escuchar, tienen como objetivo empoderarlos para que, ulteriormente, se pase a la acción.

Por último, se puede observar que la filosofía con niñas y niños sí que goza de eficacia cultural, puesto que promueve un pensamiento reflexivo más allá de lo individual, encaminado hacia un bien común.

\section{Conclusión}

A lo largo de este trabajo se ha podido ver el estudio sobre la filosofía con niñas y niños y, después, por una parte, la relación y fusión de esta con la ética del discurso y, por otra, el análisis de su eficacia cultural. 
De esta manera, se ha podido observar que tanto la filosofía con niñas y niños como la ética del discurso tienen como pilar básico el diálogo. Continuando con el análisis de las dos, hemos visto que tienen como aspiración una comunidad comunicativa ideal, en la que todos los participantes tengan voz, puedan aportar sus opiniones y sean escuchados.

Asimismo, en este estudio también pudimos ver que en ninguno de los dos casos la finalidad que se persigue es un objetivo individual o estratégico, sino que se quiere llegar a la verdad mediante la diversidad de opiniones, el entendimiento mutuo y la empatía. No obstante, los dos modelos teóricos que estamos considerando siguen las mismas pautas que cualquier debate imparcial, en el que el respeto mutuo hacia las opiniones del otro o hacia su turno de palabra son las pautas que dirigen el diálogo. Y esto es así porque tenemos responsabilidad sobre todo lo que decimos, hacemos 0 callamos, ya que tenemos la capacidad y la posibilidad de interpelarnos los unos a los otros.

Si pasamos a la otra parte analizada en este texto, la eficacia cultural de la filosofía con niñas y niños, hemos podido observar que esta tiene como finalidad última la trasformación de la realidad. Es una filosofía que se propone empoderar a niñas y niños para que, posteriormente, puedan participar activamente en sus sociedades. Para llevar a cabo este propósito se empuja a los infantes hacia un pensamiento crítico, lógico y creativo.

Como conclusión se puede decir que la filosofía con niñas y niños sí que tiene una eficacia cultural, teniendo en cuenta que se les empuja hacia un pensamiento reflexivo sobre el bien común. Sin embargo, esta filosofía puede dar la imagen de que se queda bastante en la reflexión, sin llegar a la práctica, lo que veo como una posible limitación de esta. Considero que el profesorado, después de pasar sobre el proceso de reflexión y debate con el alumnado, no solo debe empoderarlo teóricamente para que en un futuro pueda actuar para transformar la realidad, sino que deben implicarlos físicamente en casos concretos de su propia sociedad. De esta manera, aunque sean niñas y niños, se verán productivos para su entorno, lo que los empoderará más que cualquier reflexión crítica.

\section{Bibliografía}

Dussel, Enrique. 1996. "La ética de la liberación ante la ética del discurso". Isegoría: Revista de filosofía moral y política 13: 135149.

Ferrer Rodríguez, Guillermina. 2012. «Filosofía para niños. Cómo acercar la filosofía a niños y niñas». Fòrum de Recerca 17: 21-34. 
Haynes, Johanna. 2004. Los niños como filósofos. El aprendizaje mediante la indagación y el diálogo en la escuela primaria. Barcelona: Paidós Educador.

Martínez Guzmán, Vicent. 2004. «Diálogo». En Enciclopedia de paz y conflictos, editado por Mario López Martínez, 302-303. Granada: Publicaciones de la Universidad de Granada.

Nos Aldás, Eloísa, Amador Iranzo y Alessandra Farné. 2012. "La eficacia cultural de la comunicación de las ONGD: los discursos de los movimientos sociales actuales como revisión». CIC. Cuadernos de Información y Comunicación 17: 209-237.

París Albert, Sonia. 2006. La transformación de los conflictos desde la Filosofía para la Paz. Tesis doctoral. Castelló de la Plana: Universidad Jaume I.

Siurana, Juan Carlos. 2007. «La ética del deporte desde la justicia como igualdad de oportunidades en la ética del discurso». Quaderns de Filosofia i Ciència 37: 79-90. 



\section{La necesidad de una educación en el perdón en los cuentos infantiles tradicionales:}

Caperucita Roja y Blancanieves

Anca-Nicoleta Rotila al286523@uji.es 
I. Resumen

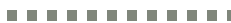

Debido a la gran importancia que tienen los cuentos infantiles en el desarrollo del carácter de los más pequeños, esta investigación tiene como objetivo el análisis de los valores que se enseñan en dos cuentos tradicionales, Caperucita Roja y Blancanieves. Para ello hemos utilizado como metodología, por una parte, el estudio de las dos historias en las versiones de los hermanos Grimm y, por otra parte, se ha recurrido a las investigaciones de autores como Bruno Bettelheim, entre otros.

De esta manera, expondremos ideas como las de familia, respeto, cuidado de los mayores o generosidad en estas narraciones. Además, destacaremos la enseñanza del castigo y la venganza en estos cuentos, así como su influencia en el comportamiento de los futuros adultos, y la necesidad de una educación en el perdón.
\end{abstract}

Palabras clave: cuentos, valores, niños y niñas, castigo, perdón.

\title{
II. Introducción
}

Cuando son pequeños, los niños y las niñas adquieren su bagaje cultural y, por tanto, su forma de entender de la vida. Por una parte, lo hacen a través del impacto que los padres causan en su carácter $y$, por otra parte, a través de la literatura infantil.

Por esta razón, el objetivo de este trabajo es el de analizar los valores que se enseñan en dos de los cuentos tradicionales por excelencia, Caperucita Roja y Blancanieves, para que, ulteriormente, podamos extraer conclusiones y proponer alternativas.

De esta manera, la estructura del siguiente trabajo empieza con el análisis de los valores que se enseñan mediante los cuentos de Caperucita Roja y Blancanieves. Posteriormente, nos adentramos en la profundidad de este estudio previo en el apartado "Resultado del análisis de los valores en Caperucita Roja y Blancanieves». Para terminar, expondremos las conclusiones y razonamientos finales.

\subsection{Caperucita Roja}

Al ser un cuento tradicional y muchas veces reinterpretado, Caperucita Roja es conocida como tal por primera vez gracias al autor francés Charles Perrault. Ya que esta versión no es muy conocida hoy en día, nos centraremos en la interpretación del mismo cuento de los hermanos Grimm.

La historia de Caperucita es ampliamente conocida: se trata de una niña cuya madre envía a visitar a su abuela enferma y en el camino se encuentra al lobo que, por su culpa, logra comerse a la abuela. Como la mayoría de los cuentos tradicionales, este también 
contiene cierta violencia que apenas se aprecia en otros relatos más recientes. Sin embargo, aparte de la violencia, como en todos los cuentos, en Caperucita Roja podemos observar ciertos valores que influyen en la mentalidad infantil.

Uno de los valores que más resaltan en este cuento es el de la familia. Aunque en ningún momento de la historia se menciona al padre de Caperucita, la niña ama a su familia, compuesta por la madre y la abuela. No obstante, cabe detenerse en este aspecto para resaltar el hecho de que la idea de familia transmitida por el cuento no es lo que se entiende por tradicional, compuesta por la madre, el padre y los hijos, sino que nos enseña una familia compuesta por la madre y su hija. Además, la preocupación de Caperucita por su abuela enferma educa a los niños en el cuidado de los mayores o de los abuelos. Asimismo, si nos centramos en la relación de la niña con su abuela, podemos hablar también de valores como la solidaridad o el respeto.

Conforme avanza la historia, puede que el valor más destacado de la trama es el de aprender a no confiar en los extraños. Hay que mencionar que, en un principio, esta historia tenía una connotación sexual muy acusada, ya que, en la versión del autor Perrault el lobo seduce a la niña con unas intenciones sexuales. Esta interpretación del siglo XV trata de enseñar a las niñas y adolescentes a no confiar en los hombres que intentan seducirlas. La versión actualizada, cuyos valores estamos analizando, tiene como finalidad enseñar a los más pequeños a no irse con extraños, y sobre todo, a no hablar con estos. Así, debido a que Caperucita le reveló la dirección de la abuelita al lobo, este pudo llegar primero y comérsela.

Otro valor relevante de nuestra historia es el de no desobedecer nunca a los padres. Recordemos que, al salir de casa, la madre de Caperucita le dijo que tuviera cuidado al pasar por el bosque y que nunca se apartase del camino; claramente, la niña no obedeció, puesto que habla con el lobo y se queda a recoger flores allí donde este le enseñó (Bettelheim, 1999: 176-191).

Al final del cuento, llegamos al momento en el que el cazador salva a la niña y a su abuela cortando la barriga del lobo. Sin embargo, el cuento no finaliza aquí, sino que a Caperucita se le ocurre que pueden ponerle piedras en la barriga y coserla de nuevo. Por consiguiente, trasluce el deseo de venganza en la niña, más allá del castigo que el lobo ya recibe por comerse a las dos féminas. Retomaremos este aspecto en las conclusiones de nuestros análisis.

\subsection{Blancanieves}

El cuento de Blancanieves, aunque en su versión original destaca por el papel de la propia protagonista, en la versión de los hermanos Grimm también adquieren gran importancia los enanitos, de forma 
que el cuento pasa a conocerse como Blancanieves y los siete enanitos.

Siguiendo el mismo patrón que en el análisis del cuento anterior, el primer valor que se destaca en esta historia es la envidia o, mejor dicho, el papel destructivo de la envidia. La madrastra de Blancanieves está celosa o tiene envidia de la niña por ser más guapa que ella. Este hecho puede servir de ejemplo positivo para los más pequeños, al enseñarles que uno se debe querer a sí mismo tal y como es, y que tener envidia solo acaba provocando efectos negativos.

Al igual que en el ejemplo anterior, conviene hablar del papel de la familia que enseña este cuento. Se trata de una familia monoparental, similar a la de Caperucita Roja; sin embargo, en este caso Blancanieves vive con su madrastra después de la muerte de su padre. A modo de inciso, consideramos que mostrar ejemplos de familias diferentes al modelo tradicional impuesto por la religión tiene un gran valor educativo.

También podemos ver que la solidaridad está muy presente, más aún si cabe en el viaje que Blancanieves ha de realizar hasta lograr su final feliz. Conviene recordar que la madrastra encarga a un cazador que mate a la niña. No obstante, este la deja escapar y entrega a la reina los pulmones y el hígado de un jabalí. Se trata, pues, de un gran ejemplo de solidaridad para los niños y las niñas, así como de una anécdota que enseña que se debe ayudar a los demás si surge la oportunidad.

Es de destacar también la generosidad con la que es recibida Blancanieves por parte de los enanitos; así como la manera en la que lo comparten todo con ella y el compañerismo que encuentra entre los propios enanitos: se ayudan, trabajan juntos, viven juntos, etc. Aun así, hay que mencionar el hecho de que Blancanieves, durante su estancia en la casa de los enanitos, se dedica a los trabajos del hogar. Encontramos aquí un ejemplo del currículum oculto que subyace a los cuentos tradicionales y que construye los roles de género, entre otros aspectos.

Al igual que en el cuento de Caperucita Roja, Blancanieves es una niña inocente que decide abrirle la puerta a la reina disfrazada, aunque los enanitos le habían dicho que no abriera la puerta a nadie. Una vez más vemos un ejemplo de las consecuencias que tiene la desobediencia hacia los padres o mayores, y de qué puede pasar cuando hablamos con extraños.

Como sucede en la mayoría de los cuentos de hadas, este acaba con la boda de Blancanieves y el príncipe, después de que este la devolviese a la vida con su beso. En contraste, la madrastra de Blancanieves fue condenada a calzar unos zapatos de hierro calentados al rojo vivo, con los que tendría que bailar hasta morir 
(Bettelheim, 1999: 207-222). Una vez más encontramos el tema del castigo, en el que profundizaremos en el siguiente apartado.

\section{Resultado del análisis de los valores en Caperucita Roja y Blancanieves}

Antes de entrar plenamente en los resultados de nuestra investigación, cabe recordar la importancia que tienen los cuentos en la vida de los infantes. Habida cuenta de la trascendencia de estas historias en el carácter de los niños y las niñas, los cuentos infantiles deben dar mucha importancia a las inquietudes de los más pequeños, darles soluciones, abordar sus problemas con seriedad $y$, al mismo tiempo, acrecentar su confianza en ellos mismos y en sus futuros (Bettelheim, 1999: 11). De esta manera, podemos apreciar que cada uno de nosotros tiene todo un bagaje de conocimientos extraídos de los llamados cuentos populares de hadas. Son historias que poco tienen que ver con la sociedad moderna, pero que ofrecen una educación moral basada en lo experiencial, así como en conceptos abstractos. "En este sentido, los cuentos de hadas tienen un valor inestimable, puesto que ofrecen a la imaginación del niño nuevas dimensiones a las que le sería imposible llegar por sí solo» (Bettelheim, 1999: 13). Asimismo, en los cuento de hadas se plantean problemas de una forma concisa, para que el niño o la niña puedan abordarla de una manera sencilla. De esta forma, se evitan las situaciones complejas, al apoyarse en personajes típicos y no únicos (Bettelheim, 1999: 14).

Al igual que en los dos ejemplos que analizamos anteriormente, Caperucita Roja y Blancanieves, un hecho destacable es que en los cuentos los personajes no son buenos y malos al mismo tiempo, como en la vida real. Cada personaje tiene su carácter bien definido y a lo largo del cuento no lo cambia en ningún momento. Así, los niños se identifican con el héroe, de tal manera que, cuantas más acciones llenas de honradez realiza un personaje, más fácil le será al infante identificarse con este. Por tanto, vemos que en la niñez no se preguntan si quieren ser buenos o malos sino que la cuestión es más bien a quién quieren parecerse. Es un acto mimético típico de la infancia (Bettelheim, 1999: 15). Además, en la gran mayoría de los cuentos tradicionales de hadas, el personaje malvado consigue el puesto del héroe por un periodo limitado. Para ejemplificar esto podemos pensar en el lobo de Caperucita Roja o la malvada madre de Blancanieves.

Sin embargo, como ya anticipamos en los dos apartados anteriores, queremos detenernos en el castigo. En todos los cuentos infantiles tradicionales, el personaje malo o negativo es castigado al acabar el cuento, lo que no podemos decir que constituya un buen ejemplo o experiencia en la educación moral del infante. Tal como 
nos explica el autor Bettelheim: "Tanto en los cuentos de hadas como en la vida real, el castigo, o el temor al castigo, sólo evita el crimen de modo relativo» (Bettelheim, 1999: 14-15). Por tanto, entendemos que el castigo aparece en cada uno de los cuentos para enseñarnos que la venganza mediante el crimen no cambiaría nada en el personaje negativo. No obstante, mediante el castigo tampoco se nos muestra una trasformación del carácter considerado como malo o negativo. Es decir, por un lado, se enseña a los niños que cualquier acto malvado o que no cumpla con unas determinadas normas será castigado gravemente. Por otra parte, independientemente del acto, se da máxima importancia al castigo y nunca al perdón.

Continuando con esta idea, si partimos de la gran influencia educativa moral que tienen los cuentos en el carácter infantil, puede deducirse que la conducta dañina o vengativa de la futura persona adulta podría tener sus antecedentes aquí, en los cuentos de hadas. Al igual que estas historias nos influyen en la idea de familia convencional, mediante el príncipe y la princesa, o que cada disputa entre grandes reyes se acaba en una guerra, se contribuye así a la idea de que debemos vengarnos de todo aquello que nos hagan y aplicar un castigo. Puede considerarse que algunas de las conductas que se pueden ver en la sociedad actual tienen parte de su germen en los cuentos tradicionales.

En relación con esta idea, en el sistema educativo actual, encontramos un gran vacío, especialmente en lo relativo al perdón. Aunque en los últimos años se ha puesto cada vez más de moda la educación en valores, este hueco del que hablamos sigue sin ser tratado y se continúan dejando de lado algunas experiencias básicas como el perdón o el arrepentimiento, entre otras. Si nos remitimos al pasado de cada uno, podríamos recordar cómo se trataba el perdón en la educación infantil, cuando la maestra o el maestro nos exigían pedir perdón por morder, pegar o cualquier otro acto similar. A pesar de ello, el perdón no se aborda desde un punto de vista más profundo, como sucede con otros valores como la solidaridad, el respeto o el amor. También parece que el tema del perdón queda relegado a los primeros años y no resurge conforme avanzamos en los años del estudio.

Según los autores María del Rosario González Martín y Juan Luis Fuentes, "el perdón es decisivo en la constitución de una persona sana, o al menos, la decisión constante de otorgarlo o no» (González Martín y Fuentes, 2012: 480). Por tanto, el perdón debería ser una de los temas prioritarios en el currículum educativo. En nuestra sociedad, en la que las emociones suelen considerarse de segundo orden o directamente ningunearse, es preciso recurrir a dinámicas tan profundas como es el perdón, en las que el niño o la niña no retengan una imagen negativa tanto del otro como de sí mismos, así 
como que aprendan a gestionar sus sentimientos de venganza y futura reparación. Sin el perdón, aunque la situación conflictiva se haya detenido, la relación no se puede curar (González Martín y Fuentes, 2012: 482).

Además, aunque el ámbito familiar es reconocido como un lugar ético en el que se deben aprender los valores básicos desde los primeros años de vida, el ámbito educativo, debido a la gran cantidad de relaciones interpersonales y sociales que se establecen en las escuelas, debe involucrarse en la enseñanza del perdón. «Como toda experiencia humana el perdón necesita ser educado y por ello, ni la familia ni la escuela pueden ignorarlo» (González Martín y Fuentes, 2012: 485). Por tanto, toda educación debe enseñar, aparte de la transformación pacífica de conflictos mediante la comunicación, la trasformación sana y constructiva de las personas, la comunidad y la sociedad.

Así, en el proceso educativo el educador debe ejercer de facilitador que ayude al alumnado a abordar de frente la acción negativa que ha recibido, de manera que pueda valorarla sin justificarla o relativizarla. El educador debe partir de la idea del conflicto como algo inherente a la condición humana, y por tanto, imposible de evitar. Aunque un conflicto puede resultar una experiencia negativa, se ha de tratar como una oportunidad de cambio. Como la autora Sonia París Albert (2009: 21) nos dice:

Así, siguiendo a Francisco Muñoz, se considera que el carácter positivo o negativo de los conflictos depende de los medios que son usados para su regulación. En este sentido, el conflicto será negativo si es gestionado con violencia y positivo si es regulado con la práctica de medios pacíficos. En el primero de los casos su carácter negativo será fruto de las consecuencias destructivas que la violencia produce, a diferencia el segundo en el cual será positivo debido al cuidado de las relaciones que se alcanza cuando se actúa de manera pacífica.

Es más, "enfrentarse al mal cometido y sus consecuencias es ocasión de crecimiento personal» (González Martín y Fuentes, 2012: 486).

Si profundizamos en la necesidad de educar en el perdón, podemos hablar de recuperación de la dignidad y de la esperanza. Según nos dice la autora Arendt (1995: 29), el perdón nos aleja de la venganza y "logra dar lugar a un nuevo comienzo allí donde todo parecía haber concluido». Y esto se debe a que, como personas $y$, por tanto, actores, somos capaces de perdonar y arrepentirnos (Hilb, 2015: 46), más aún cuando el perdón y la reconciliación se traducen en trabajo conjunto por un mundo común mejor (Hilb, 2015: 52). 
Si abundamos un poco más en el tema, podemos afirmar que el perdón y la promesa son facultades sin las que la acción no es posible (López, 2011: 269); aquí entendemos por acción:

... ese poder de hacerse parte del mundo espontáneamente; de comenzar procesos originarios como la existencia humana misma. La acción, en estos términos, es una actividad enteramente positiva, pues desvela la posibilidad del comienzo que es inherente al nacimiento, al origen, de la humanidad.

Hannah Arendt nos explica que no podemos retroceder el tiempo para deshacer un mal ya cometido, y tampoco podemos garantizar que ese mal no se volverá a hacer. Pero ante estas imposibilidades, los únicos remedios son el perdón y la promesa (López, 2011: 270). Estamos hablando de un perdón que tiene que ver con el amor, no un amor romántico, sino un amor de amistad que, junto con el perdón, se entiende como el respeto al prójimo (López, 2011: 277). Esto se debe a que, según la autora Arendt «el perdón solo puede darse en la relación directa entre quien perdona y quien es perdonado, ya que el perdón y la promesa realizados en soledad $\mathrm{o}$ aislamiento carecen de realidad $\mathrm{y}$ no tienen otro significado que el de un papel desempeñado ante el yo de uno mismo» (Arendt, 2009: 257). A partir de esta última idea, debemos mostrar nuestro desacuerdo con la autora, al considerar que el perdón es una acción personal liberadora con dos vertientes. Por una parte, uno ha de perdonar a su prójimo; por otra parte (quizá la más importante), uno tiene la posibilidad de liberar su conciencia al perdonar.

En suma, esta propuesta no se inscribe en un marco educativo en el que se deban dar "clases de perdón» o enseñarse este valor de una manera forzada. Consideramos que, al igual que otros muchos valores aprendidos desde la infancia, el perdón se ha de trabajar a través de la literatura, desde los cuentos infantiles.

\section{Conclusiones}

Dada la gran importancia que tienen los cuentos infantiles en el desarrollo del carácter de los niños, hemos empezado por analizar los valores transmiten, mediante dos de los cuentos más conocidos, Caperucita Roja y Blancanieves.

Puesto que la versión inicial de Caperucita Roja del autor Charles Perrault no parece estar muy adaptada a los tiempos actuales y tiene cierta inclinación hacia lo sexual, nuestro análisis se basó en la versión adaptada de los hermanos Grimm. Aunque la violencia es patente, como sucede en casi todos los cuentos de este tipo, en la historia de Caperucita Roja también encontramos valores bien ejemplificados que pueden establecer una base educativa para los 
niños y las niñas. De esta manera, a lo largo del cuento encontramos

enseñanzas como el hecho de no confiar en los extraños y no desobedecer a los padres, así como el respeto y el cuidado de los mayores o los abuelos, o bien una idea de familia muy frecuente en nuestros días.

En cuanto al análisis de los valores y enseñanzas presentes en el cuento de Blancanieves, el tema principal de esta historia es cómo la envidia puede llegar a ser destructiva tanto para uno mismo como para los que le rodean. Así pues, destacamos las enseñanzas de solidaridad, generosidad y ayuda al prójimo cada vez que se tiene la oportunidad. Como similitudes entre los dos cuentos analizados, vemos que en ambos se aborda el tema de la familia monoparental y las repercusiones que pueden tener conductas como hablar con extraños o confiar en ellos.

No obstante, si algo podemos destacar no solo en estas dos historias, sino también en la gran mayoría de los cuentos de hadas, es el castigo o la venganza. Partiendo de la afirmación de que gran parte de nuestro bagaje de conocimientos se adquiere durante la infancia mediante los cuentos infantiles, es fácil deducir que parte de estos conocimientos están determinados también por la idea de castigo. Es más, los niños y las niñas se suelen identificar con el héroe, un héroe que siempre gana mediante la venganza o el castigo del personaje negativo, nunca por medio del perdón. Aunque puede parecer una enseñanza mínima y de importancia cuestionable, al igual que las niñas sueñan desde pequeñas con casarse con su príncipe azul debido a la influencia de los cuentos de hadas, consideramos que la idea del castigo de las historias infantiles desempeña un importante papel en los caracteres que predominan en nuestra sociedad adulta actual.

Muy ligado a este problema está la ausencia del tratamiento del perdón dentro del ámbito educativo. Esta experiencia básica entre personas debería estar presente en la educación de los más pequeños desde una edad temprana. De esta manera, al igual que se da mucha importancia a valores como el respeto o la solidaridad, consideramos necesaria la educación en el perdón.

Por una parte, la libertad individual, aparte de otros pilares fundamentales, también tiene como base el perdón, especialmente cuando hablamos de niños cuyo entorno más próximo son las escuelas, en las que abundan las relaciones sociales y, por tanto, los conflictos. Por otra parte, al perdonar podemos hablar de la recuperación de la dignidad y la esperanza, al confiar en la trasformación positiva del conflicto.

Para finalizar, consideramos que la educación en el perdón es de vital importancia en nuestra sociedad, pero no de una manera forzada, sino, al igual que otros valores y enseñanzas que interiorizamos desde pequeños mediante los cuentos, y que se 
quedan con nosotros para toda la vida, el perdón se ha de aprender a través de la literatura infantil.

\section{Bibliografía}

Arendt, Hannah. 1995. De la historia a la acción. Barcelona: Paidós.

Arendt, Hannah y Manuel Cruz. 2009. La condición humana. Barcelona: Paidós.

Bettelheim, Bruno. 1999. Psicoanálisis de los cuentos de hadas. Barcelona: Crítica.

González Martín, María del Rosario y Juan Luis Fuentes. 2012. "Los límites de las modas educativas y la condición humana. Un hueco de la educación de las grandes experiencias: el perdón». Revista Española de Pedagogía 253: 479-493.

Hilb, Claudia. 2015. "Justicia, reconciliación, perdón», En Lesa humanidad: Argentina y Sudáfrica: reflexiones después del Mal, editado por Claudia Hilb, 53-74. España: Katz.

López, María. 2011. "La reversibilidad del tiempo, el perdón y la promesa en Hannah Arendt». Investigaciones fenomenológicas: Anuario de la Sociedad Española de Fenomenología 3: 265-278.

París Albert, Sonia. 2009. Filosofía de los conflictos. Una teoría para su transformación pacífica. Barcelona: Icaria. 


\section{Las distopías en las series de televisión contemporáneas:}

una aproximación

Francisco Giménez Mateu

al362643@uji.es 
En las páginas que siguen me propongo presentar el proyecto preparado para el desarrollo de mi tesis doctoral enmarcada en el programa de doctorado en Estudios Interdisciplinares de Género. Una tesis doctoral que se centrará en el concepto filosófico de distopía a través de un análisis tanto literario como audiovisual de las ideas de utopía y distopía a lo largo de la historia. Para ello, estudiaré desde la utópica La República, de Platón, hasta la distópica 1984, de George Orwell; y desde la película Metrópolis, de Fritz Lang, hasta la serie de televisión El cuento de la criada, creada por Bruce Miller. En este artículo planteo una introducción a dicha tesis doctoral, además de un acercamiento a su metodología y objetivos propuestos: encontrar esos puntos comunes entre las producciones utópicas y distópicas analizadas que nos conduzcan a componer una noción integral de distopía para tratar de com-prender una definición de dicho término y des-cubrir si las relaciones de género, entre otras cosas, pueden definir aquel tipo de sociedades que damos en llamar distópicas. Por eso, como el texto siguiente se trata de una proyección, el lector puede echar en falta apartados tales como «Resultados» o "Conclusiones». Esto es así porque la finalidad de las próximas líneas es tan solo esbozar un proyecto que irá cogiendo forma en un futuro cercano y que, espero, poder describir en próximos números de Fòrum de Recerca.

Palabras clave: distopía, utopía, estudios de género, series de televisión, análisis literario, análisis audiovisual

\section{Introducción}

El espíritu de la distopía, siempre pesimista, se ha generalizado tanto en nuestro tiempo como en su momento lo hizo el espíritu, siempre optimista, de la utopía (Núñez Ladevéze, 1986: 122). Si podemos decir que la utopía aparece ligada a momentos históricos determinados, a momentos de crisis (Servier, 1969: 228), lo mismo podemos decir, pienso, de la distopía. Una distopía y utopía que quedan ligadas, también, en su definición. Porque si intentar definir la idea de utopía es, o puede ser, tanto o más inútil que definir el significado de filosofía (Blanco Martínez, 2000: 11), lo mismo podemos decir de la distopía. Y es que no fue hasta 2014 cuando José María Merino preparó este término para su entrada en el Diccionario de la Lengua Española (Morán Bueno, 2014; Costa Vila, 2014) y la definió así: «representación ficticia de una sociedad futura de características negativas causantes de alienación humana» (DLE 2014, 814). 
La primera referencia de la palabra utopía, la encontramos (con

todas sus ambigüedades) en Tomás Moro (Mumford, 2013: 9), en su libro De optimo reipublicae statu deque nova insula Utopía, publicado en 1516 (Trousson, 2011: 10). Y la primera referencia de la palabra distopía, la encontramos (aunque no significa exactamente lo que hoy entendemos por la palabra) en John Stuart Mill, en un discurso parlamentario en 1868: "It is, perhaps, too complimentary to call them utopians, they ought rather to be called dys-topians [...]» (Aldridge, 1984: 8). Con esto quería llegar a interpretar tanto a la utopía como a la distopía como elementos ligados a su propio tiempo, un tiempo que identifico con la modernidad y con la posmodernidad.

Aunque mitos hay en todos los pueblos del mundo, "utopías» (y añado, por lo tanto, "distopías») solo se han forjado en Occidente, debido, sobre todo, a esa concepción judeocristiana del mundo que contrapone lo que es a lo que debe (o debería) ser: ese sentimiento profundo de una meta por alcanzar (Cappelletti, 1966: 3; Servier, 1969: 230), ese deseo del "deber ser», ese afán por lo justo del que habla Martin Buber (1955: 17). Es, pues, esa idea de que hay un deber ser implícito en el ser mismo y que, además, puede explicitarse especulativamente, lo que introduce el rasgo utópico en el pensamiento (Núñez Ladevéze, 1986: 111). Jean Servier (1969: 230) describe dos grandes características de toda utopía: la descripción de una ciudad considerada perfecta y una crítica del orden social. Una crítica que, mediante la comparación con la realidad, muestra sus defectos (Núñez Ladevéze, 1986: 112). De ahí la definición que hace de utopía Martin Buber (1955: 17): «una expresión de algo que no existe, que solo es imaginario». Una expresión que, como ya he dicho, supone una crítica al presente y un intento de descubrir otras alternativas (Mumford, 2013: 10-11; Núñez Ladevéze, 1986: 114). Una actitud crítica que aparece ante el disgusto del modo de ser actual del mundo: un disgusto que nos prepara para comprender lo que este mundo tiene de equivocado (Buber, 1955: 18).

Si la modernidad, ya agotada, ha dejado paso a la posmodernidad (Díaz, 1999: 13, 31), lo mismo puede decirse, creo, de la utopía y la distopía: la utopía, ya agotada, ha dejado paso a la distopía. Una utopía que, relacionada en la modernidad con la dialéctica histórica, parecía justificar y legitimar cualquier presente (Devueau, 1961: 31-32). Además, como apunta Lewis Mumford (2013: 12-14): la gran mayoría de las utopías clásicas tienen en su base elementos cerrados, inflexibles y totalitarios. De ahí las debilidades esenciales de la utopía que, pienso, son superadas, ahora, con la distopía. Luis Núñez Ladevéze (1986: 120) afirma, en este sentido, que "el desencanto ha sustituido al candor de la utopía primigenia». Y Jean Servier (1969: 230) que «semejante angustia es a 
menudo generadora de sueños compensadores». Así pues, esa sensación de desánimo, pesimismo y desencanto desemboca en la distopía ( $y$, también, creo, en la posmodernidad): un instrumento poderoso con el que poder exponer el disentimiento, la protesta y el temor (Núñez Ladevéze, 1986: 121).

\section{Objetivos}

Como pienso que no pueden, a priori, separarse los conceptos de utopía y distopía para estudiarlos, he considerado totalmente necesario un acercamiento al concepto de distopía a través del de utopía. Por ello, mi entrada al mundo de la distopía se llevará a cabo a partir del de la utopía. Y para abrir las puertas de ese mundo utópico, tomaré las llaves de aquellas obras que se han considerado como fundantes del género utópico, como Utopía, de Tomás Moro (quien, dicho sea de paso, inventa el neologismo utopía); Ciudad del Sol, de Tommaso Campanella, o Nueva Atlántida, de Francis Bacon. También pasaré revista al contexto histórico que acompaña a estas obras porque toda obra nace en un tiempo, y tiempo y obra, pienso, no pueden leerse por separado. Luego de haber hecho una lectura literaria e histórica de la utopía, estaré preparado para entrar en el reino de la distopia. Una distopía (tema central de mi tesis doctoral) que, también, como la utopía, nace en un determinado tiempo. Por eso, haré el acercamiento a la distopía desde dos perspectivas. Uno, una perspectiva literaria (analizando cómo se pasa de un pensamiento utópico y optimista a un pensamiento distópico de corte pesimista) comparando la literatura utópica con la literatura distópica y descubriendo cómo, necesariamente, las obras utópicas (debido a sus limitaciones) nos conducen a las obras distópicas. Dos, una perspectiva audiovisual, analizando películas y series de televisión distópicas, porque si la distopía forma parte de un tiempo concreto (como defiendo) ese tiempo es el tiempo del cine, el tiempo de las series de televisión. Necesariamente, pues, haré un repaso de las primeras películas con una temática distópica clara, como puede ser, por ejemplo, Metrópolis, de Fritz Lang, en 1929, para observar cómo se pueden complementar la literatura y lo audiovisual para llevarnos de la mano a una noción de la distopía más precisa.

A continuación, entraré de lleno en la parte más específica de toda mi tesis doctoral: un análisis audiovisual de algunas series de televisión contemporáneas para desembocar en cómo se nos muestra, qué mecanismos utiliza y por qué nos afecta emocionalmente el contenido audiovisual de dichas series de televisión. Me gustaría comentar que la elección de estas no es arbitraria y que se fundamenta en un objetivo claro: descubrir cómo nos pueden acercar las series de televisión a un contenido 
existencial, a valores morales y a conceptos filosóficos. Además, para dotar a todo este estudio de una perspectiva de género, dicha elección se meditará y mucho. De ahí que pueda anticipar, por ahora, El cuento de la criada, serie de televisión (además de obra literaria) que nos narra una sociedad donde la mujer ha quedado relegada a un segundo plano.

Con lo dicho, puedo preludiar que mi tesis doctoral contará con el siguiente objetivo general: analizar la idea de distopía en la actualidad a través de un estudio audiovisual de algunas series de televisión actuales como El cuento de la criada con el fin de descubrir esos elementos comunes que hacen que podamos llamarlas "distópicas» para des-velar, desde allí, una teoría y definición de la distopía. Además, también trabajaré estos otros objetivos específicos: primero, desarrollar una historia de la "utopía» a partir de sus textos más significativos para encontrar esos elementos comunes que hacen que se las pueda llamar "utopías» e imaginar una definición o una teoría que pudiera incluirlas a todas. Segundo, estudiar una historia de la «distopía» a través de un análisis tanto de sus aportes literarios como de sus producciones audiovisuales más significativas para encontrar, igual que en el caso anterior, esos elementos comunes que hacen que se las pueda llamar "distopías» e imaginar una definición o una teoría que pudiera incluirlas a todas. Tercero, encontrar una identificación histórica tanto de la utopía como de la distopía para comprender por qué aparecen en un determinado momento y no en otro y relacionarlas, así, con su tiempo y entre ellas mismas. Y cuarto, llevar a cabo un análisis literario de todas las obras utópicas y distópicas citadas, ademá, de complementarlo con un análisis audiovisual de algunas de sus producciones audiovisuales (en este caso, algunas series de televisión) para entender cómo se vierten las ideas literarias en el mundo audiovisual, cómo se complementan y cómo pueden ayudarnos a entender algunos conceptos (en este caso, la distopía) de una forma más completa.

\section{Metodología}

Con lo ya anticipado, queda claro que para estudiar el concepto de distopía, será necesario, a su vez, estudiar el concepto de «utopía». Como ya he dicho, entiendo la distopía como un cambio histórico inevitable que nos lleva a movernos desde la utopía hasta la distopía. Por eso, mi investigación acerca de la distopía arrancará necesariamente con una aproximación a la utopía para, una vez formada una idea de qué es, poder movernos hasta la distopía.

Para rastrear la utopía me centraré, primero, en obras filosóficas, por ser estas su primera manifestación. Echaré mano, pues, tanto de obras clásicas que pueden considerarse utópicas 
(como La República, de Platón), pasando por otras obras que se considera que fundaron la utopía como género literario (como Utopía, de Tomás Moro; Ciudad del Sol, de Tommaso Campanella, o Nueva Atlántida, de Francis Bacon), hasta llegar al siglo xx (con obras como Altneuland, de Theodor Herzl, o Una utopía moderna, de H. G. Wells). No está de más decir que también analizaré el contexto en el que aparecen todas estas obras para entender por qué aparecen en ese momento y no en otro.

Una vez llevado a cabo este recorrido histórico y literario a través de la utopía, avanzaré hasta la distopía y sus manifestaciones. En este caso, para observar cómo se pasa del pensamiento utópico al pensamiento distópico, echaré mano de algunas obras que se han considerado fundantes de la distopía como género literario como Un mundo feliz, de Aldous Huxley; 1984, de George Orwell, o Fahrenheit 451 , de Ray Bradbury. Tras esto, también pasaré a revisar algunas otras obras distópicas para complementar la lectura de las anteriores como La fe de nuestros padres, de Philip K. Dick; La pianola, de Kurt Vonnegut, entre otras. Acompañaré Todos sobre Zanzíbar, de John Brunner, en este recorrido literario, al igual que en el caso anterior, de un estudio histórico, en el que observaré el tiempo en el que aparecen estas obras, para entender por qué aparecen entonces, en ese determinado momento y no en otro.

Y tras este prometedor análisis literario e histórico de los conceptos de utopía y distopía, y con una idea ya formada de qué es eso que hoy en día llamamos distopía, centraré mi análisis, ya específicamente, en cómo se muestra en las series de televisión (parte novedosa de mi tesis doctoral). Así pues, a través de un análisis audiovisual (cuya metodología desarrollaré a lo largo del trabajo), estudiaré tanto la estética de las series de televisión (a través de un análisis de su lenguaje y narrativa audiovisual) como la parte sociopolítica y ética (a través de un análisis de los componentes sociales, políticos y éticos inherentes a sus tramas y especialmente de las relaciones de género), de modo que relacionaré, así, sociedad, política, ética y estética para ver cómo se manifiestan en conjunto en pantalla.

Por eso, para hablar de la metodología que utilizaré a lo largo de toda mi tesis doctoral, iré por partes, ya que no solo será una, sino varias, que se ayudarán y complementarán entre sí. Primero (y por orden cronológico) llevaré a cabo un análisis literario de algunas de las obras utópicas y distópicas más significativas, algunas de las cuales ya he mencionado: Utopía, Ciudad del Sol o Nueva Atlántida, por una parte, y Un mundo feliz, 1984 o Fahrenheit 451, por otra, para ver qué pueden decirnos y cómo se relacionan entre ellas. Asimismo, echaré mano de textos como Principios de análisis literario, de Cesare Segre (1985); Estructura del texto artístico, de Yuri M. Lotman (1988); Teoría de la novela. Antología de textos del 
siglo Xx, de Enric Sullà (1996); Manual de crítica literaria contemporánea, de Fernando Gómez Redondo (2008), Conceptos de crítica literaria, de René Wellek (1968); Literatura comparada: principios y métodos, de María José Vega y Neus Carbonell (1998), o Historia social de la literatura y el arte, de Arnlod Hauser (1978; 1993a; 1993b) entre muchos otros. Todo este análisis literario irá acompañado de un análisis histórico. Así pues, también estudiaré, además de los conceptos utopía y distopía, los de modernidad y posmodernidad como referentes históricos en los que aparecen tanto la utopía como la distopía como género literario. Para esto me ayudaré de algunas obras como ¿Qué es Modernidad?, de Bolivar Echeverría (2009); Todo lo sólido se desvanece en el aire. La experiencia de la modernidad, de Marshall Berman (1989); Modernidad y posmodernidad, de Armando Roa (1995); El debate modernidad-posmodernidad, de Nicolás Casullo (2004); Los orígenes de la posmodernidad, de Perry Anderson (2000); La posmodernidad, de Hal Foster (2008); Posmodernidad, de Esther Díaz (1999); La condición de la posmodernidad: investigación sobre los orígenes del cambio cultural, de David Harvey (1998); De la mano de Alicia: Io social y lo político en la postmodernidad, de Boaventura de Sousa Santos (1998), o Las ilusiones del posmodernismo, de Terry Eagleton (1997), entre otras.

Además, analizaré aspectos sociopolíticos, éticos y estéticos tanto de películas como de series de televisión distópicas. Parte del material que he utilizado para ello está compuesto de libros como Manual básico de lenguaje y narrativa audiovisual, de Federico Fernández Díez y José Martínez Abadía (1999), para aprehender las reglas básicas del mundo audiovisual; Estética del cine. Espacio filmico, montaje, narración, lenguaje, de Jacques Aumont et al. (2008), para entender todos los elementos observables en una película o serie de televisión, o Hacia una filosofía de la fotografía, de Vilém Flusser (1990) que toma la imagen como algo susceptible de interpretación. Así como De la literatura al cine. Teoría y análisis de la adaptación, de José Luis Sánchez Noriega (2000); Leer el cine. La teoría literaria en la teoría cinematográfica, de José Antonio Pérez Bowie (2008); Las cinco claves del cine, de Joseph Mascalli (1990); ¿Qué es una buena película?, de Laurent Jullier (2006); Nuevos conceptos de la teoría del cine. Estructuralismo, semiótica, narratología, psicoanálisis, intertextualidad, de Robert Stam et al. (1999); Sociología del cine. La apertura para la historia de mañana, de Pierre Sorlin (1985), o Veinte lecciones sobre la imagen y el sentido, de Guy Gauthier (1996).

En cuanto a la metodología en sí, para el análisis del material audiovisual me ayudaré (no de todos los elementos, sino tan solo de los que considere necesarios) de una aproximación desde múltiples perspectivas, combinando el análisis cuantitativo y cualitativo que 
expone Laura Cortés-Selva en su artículo «Fotografía y series de televisión. Metodología para el análisis del estilo visual televisivo» (2016: 135-150). Este análisis, en resumen, se divide en tres niveles: en primer lugar, descriptivo (descripción del estilo visual con un análisis del plano individual) en el que se observarán, entre otras, variables formales (la duración del plano en segundos, la escala de plano, la tipología de movimientos de cámara, el diseño lumínico y el diseño colorimétrico), variables de contenido (las localizaciones dominantes, los espacios interiores o exteriores) y variables temporales (si es de día o de noche o si es presente, pasado o futuro), mediante una metodología cuantitativa (descriptiva, susceptible de medición). En segundo lugar, un nivel analítico en el que se analizarán el tiempo y el movimiento, es decir, la escena y la secuencia, para encontrar significados y posibles funciones que cumplen los patrones estilísticos hallados mediante una metodología cualitativa (o interpretativa). Y en tercer lugar, el histórico, que se encaja en el contexto de su tiempo y que tiene en cuenta las condiciones económicas, tecnológicas, los estándares de la industria y ciertos códigos estético-culturales de un determinado momento histórico, ya que se entiende que el estilo es el resultado de una conjunción de todo ello.

Finalmente, introduciré la perspectiva de género en el análisis. En la distopía existe, implícitamente y explícitamente, el elemento de las relaciones de poder, que pueden interpretarse en clave de género (Turbet, 2003: 14). Además, la perspectiva de género nos des-vela que hay ciertos términos que son construidos (Laqueur, 1994: 2125), que hay productos que son sociales y culturales (Ortner y Whitehead, 1981: 1) y es exactamente adonde quiero llegar: cómo se muestra en las series de televisión esa construcción social y cultural que es el género, ya que estas han alcanzado en los últimos años una relevancia cultural y social enorme (Muñoz Fernández, 2016: 70). Además, la idea es analizar cómo se muestran las relaciones de género en las series escogidas y cómo rompen o los modelos y estereotipos de género que nos han querido vender como naturales pero que, en realidad, son construcciones históricas al servicio de intereses políticos y sociales (Butler, 1990: 39-40). Así pues, con el visionado de estas series de televisión no solo me centraré en el análisis de los modelos y estereotipos de género que arrastramos sino que, además, en si estas contribuyen a perpetuar dichos modelos y estereotipos o si, por el contrario, nos ayudan a romperlos, y, si es así, cómo.

\section{Estructura y planificación}

Para acabar con la exposición de mi proyecto, explicaré brevemente la estructura y la planificación de mi tesis doctoral. Con 
la finalidad de abordar el objetivo general y los objetivos específicos a través de la metodología propuesta, el orden lógico de mi tesis será el siguiente: en la primera parte, a modo de presentación, trataré, desde la literatura, la historia y lo audiovisual la idea de utopía y de distopía. En la segunda parte, la más personal de este trabajo, y que sigue desde la primera, tratará de profundizar filosóficamente en el concepto de distopía a partir del análisis y la reflexión del lenguaje y la narrativa audiovisual que nos muestran algunas series de televisión en la actualidad.

En cuanto a la planificación, la tesis doctoral está planteada para desarrollarla, a tiempo completo, durante tres años. Dado que la propuesta esbozada tiene una lógica interna que no escapa a sus partes y no está planteada desde apartados independientes, sino a partir de apartados que se apoyan y completan, no se podrá plantear una planificación cerrada, iré tratando los distintos temas a lo largo de estos tres años. Si se trata de ser lo más ajustado posible, podría anticipar que el primer año estará dedicado a la revisión bibliográfica, lectura y revisión de los conceptos de utopía y distopía en general, además de la investigación de las obras escritas y películas realizadas de temática utópica y distópica más significativas de la historia. Así, el segundo año arrancará con una revisión filmográfica y el visionado a fondo de las películas de temática distópica; en este punto introduciré la metodología propuesta propuesta para el análisis audiovisual (sociopolítico, ético y estético). Así pues, podré profundizar en todas esas películas para extraer tanto su sentido ético como estético y lanzarme a la interpretación. Y con todo, a partir del tercer año, visionaré las series seleccionadas para tener una sólida base en cuanto a novelas (textos literarios), películas (material audiovisual) utópicas y distópicas por una parte y haber desarrollado la metodología propuesta y seguirla a lo largo del trabajo, por otra. De esta manera, empezaré a trabajar en detalle las series de televisión. Para terminar, dedicaré los últimos meses a leer, revisar, corregir y acabar de definir y dar forma a toda la tesis doctoral en conjunto.

\section{Bibliografía}

Aldridge, Alexandra. 1984. The Sceintific World View in Dystopia. Michigan: UMI Research Press.

Anderson, Perry. 2000. Los orígenes de la posmodernidad. Barcelona: Anagrama.

Aumont, Jacques, Alain Bergala, Michel Marie y Marc Vernet. 2008. Estética del cine. Espacio fílmico, montaje, narración, lenguaje. Buenos Aires: Paidós. 
Berman, Marshall. 1989. Todo lo sólido se desvanece en el aire. La experiencia de la modernidad. Buenos Aires: Siglo XXI.

Blanco Martínez, Rogelio. 1999. La ciudad ausente. Utopía y utopismo en el pensamiento occidental. Madrid: Akal.

Buber, Martin. 1955. Caminos de Utopía. Ciudad de México: Fondo de Cultura Económica.

Butler, Judith. 1990. El género en disputa. El feminismo y la subversión de la identidad. Ciudad de México: Paidós.

Cappelletti, Ángel J. 1966. Utopías: antiguas y modernas. Quito: J. M. Cajica.

Casullo, Nicolás, ed. 2004. El debate modernidad-posmodernidad. Buenos Aires: Retórica.

Cortés-Selva, Laura. 2016. "Fotografía y series de televisión. Metodología para el análisis del estilo visual televisivo». index.comunicación. Revista científica en el ámbito de la Comunicación Aplicada 6 (2): 135-150.

Costa Vila, Jordi. 2014. "El tiempo de la distopía». El País, 10 de octubre.

https://elpais.com/cultura/2014/10/01/babelia/1412173689_53 9421.html

Díaz, Esther. 1999. Posmodernidad. Buenos Aires: Biblos.

Duveau, Georges. 1961. Sociologie de l'utopie es autres «essais». París: Presses Universitaires de France.

Eagleton, Terry. 1997. Las ilusiones del posmodernismo. Buenos Aires: Paidós.

Echeverría, Bolivar. 2009. ¿Qué es Modernidad?. Ciudad de México: Universidad Nacional Autónoma de México.

Fernández Díez, Federico y José Martínez Abadía. 1999. Manual básico de lenguaje y narrativa audiovisual. Barcelona: Paidós.

Flusser, Vilém. 1990. Estética del cine. Espacio fílmico, montaje, narración, lenguaje. Ciudad de México: Trillas.

Foster, Hal, ed. 2008. La posmodernidad. Barcelona: Kairós.

Gauthier, Guy. 1996. Veinte lecciones sobre la imagen y el sentido. Madrid: Cátedra.

Gómez Redondo, Fernando. 2008. Manual de crítica literaria contemporánea. Madrid: Castalia

Harvey, David. 1998. La condición de la posmodernidad: Investigación sobre los orígenes del cambio cultural. Buenos Aires: Amorrortu. 
Hauser, Arnold. 1978. Historia social de la literatura y el arte 1. Madrid: Labor.

-. 1993a. Historia social de la literatura y el arte 2. Madrid: Labor.

-. 1993b. Historia social de la literatura y el arte 3. Madrid: Labor.

Jullier, Laurent. 2006. ¿Qué es una buena película? Barcelona: Paidós.

Laqueur, Thomas. 1994. La construcción del sexo. Cuerpo y género desde los griegos hasta Freud. Madrid: Cátedra.

Lotman, Yuri M. 1988. Estructura del texto artístico. Madrid: Istmo.

Mascalli, Joseph. 1990. Las cinco claves del cine. Buenos Aires: Sindicato de la Industria Cinematográfica Argentina.

Morán Bueno, David. 2014. «Literatura distópica: cuando el futuro es una pesadilla». $A B C, 5$ de octubre.

https://www.abc.es/cultura/libros/20140805/abci-literaturadistopica-201408041809.html

Mumford, Lewis. 2013. Historia de las utopías. Logroño: Pepitas de calabaza.

Muñoz Fernández, Horacio. 2016. "¿Son arte las series de televisión?». index.comunicación. Revista científica en el ámbito de la Comunicación Aplicada 6 (2): 69-82.

Núñez Ladevéze, Luis. 1986. "Sobre el proceso de la utopía a la distopía». Revista de Estudios Políticos 52: 111-123.

Ortner, Sherry B. y Harriet Whitehead. 1981. "Introduction: Accounting for Sexual Meanings». En Sexual Meanings: The Cultural Construction of Gender and Sexuality, editado por Sherry B. Ortner y Harriet Whitehead, 1-28. Cambridge: Cambridge University Press.

Pérez Bowie, José Antonio. 2008. Leer el cine. La teoría literaria en la teoría cinematográfica. Salamanca: Ediciones Universidad de Salamanca.

RAE. 2014. Diccionario de la Lengua Española. 23ạ edición. Barcelona: Espasa.

Roa, Armando. 1995. Modernidad y posmodernidad. Santiago de Chile: Andrés Bello.

Sánchez Noriega, José Luis. 2000. De la literatura al cine. Teoría y análisis de la adaptación. Barcelona: Paidós.

Segre, Cesare. 1985. Principios de análisis del texto literario. Barcelona: Crítica.

Servier, Jean. 1969. Historia de la utopía. Caracas: Monte Ávila. 
Sorlin, Pierre. 1985. Sociología del cine. La apertura para la historia de mañana. Ciudad de México: Fondo de Cultura Económica.

de Sousa Santos, Boaventura. 1998. De la mano de Alicia: lo social y lo político en la postmodernidad. Bogotá: Siglo del Hombre, Facultad de Derecho de la Universidad de los Andes y Uniandes.

Stam, Robert, Robert Burgoyne y Sandy Flitterman-Lewis. 1999. Nuevos conceptos de la teoría del cine. Estructuralismo, semiótica, narratología, psicoanálisis, intertextualidad. Barcelona: Paidós.

Sullà, Enric. 1996. Teoría de la novela. Antología de textos del siglo $x x$. Barcelona: Crítica.

Trousson, Raymond. 2011. «Tomás Moro, padre de la utopía moderna». En Utopía, Tomás Moro, 9-25. Madrid: Círculo de Bellas Artes.

Turbet, Silvia. 2003. "La crisis del concepto de género». En Del sexo al género. Los equívocos de un concepto, editado por Silvia Turbet, 7-38. Madrid: Cátedra.

Vega, María José y Neus Carbonell. 1998. La literatura comparada: principios y métodos. Madrid: Gredos.

Wellek, René. 1968. Conceptos de crítica literaria. Caracas: Ediciones de la Biblioteca de la Universidad Central de Venezuela. 


\section{Simbolismo vegetal en la pintura prerrafaelita}

Amapola y adormidera: protagonistas en el estereotipo artístico de la femme fatale

Noelia Alemany Mesas alemanyn@uji.es 
I. Resumen

\begin{abstract}
1. Resumen
Los elementos naturales plasmados en el lienzo han constituido, desde siempre, un aspecto notable para la pintura. Sin embargo, es durante el prerrafaelismo -movimiento artístico desarrollado durante la segunda mitad del siglo XIX- cuando empieza a cobrar importancia la naturaleza gracias al simbolismo que adquiere cada especie vegetal representada, un simbolismo que ayudaba a entender el significado de la obra.

Este estudio pretende centrar su atención en la amapola o adormidera, y para ello se analizan dos figuras femeninas de gran relevancia dentro de la pintura decimonónica: Ofelia e Isabella. A través de estos ejemplos, se procura mostrar tanto el simbolismo vegetal hallado en dichas pinturas, como los conocimientos que los prerrafaelitas tenían en otros campos de estudio diferentes a este. Por otra parte, la elección de ambos personajes femeninos no solo permite el análisis de los elementos naturales que aparecen en las obras, sino el estudio del nuevo modelo iconográfico femenino implantado por los prerrafaelitas: la femme fatale.
\end{abstract}

Palabras clave: prerrafaelismo, Ofelia, Isabella, femme fatale, amapola.

\title{
II. Introducción
}

El estudio planteado pretende analizar la representación pictórica de la amapola o adormidera en un contexto prerrafaelita donde los autores, además de introducir este tipo de elementos naturales, también les proporcionaban un simbolismo que ayudaba a esclarecer el significado de las obras. En este caso, el análisis de esta especie vegetal parte de dos personajes femeninos: Ofelia e Isabella. Por un lado, el personaje secundario de Ofelia en la gran tragedia de William Shakespeare se ha convertido en una de las figuras más emblemáticas del género pictórico y ha servido para mostrar una faceta literaria entre los artistas de la época. Por otro lado, la presencia de Isabella muestra nuevamente un gran interés por la literatura, concretamente por la poesía, pero también por los clásicos, pues dicho poema está basado en el Decameron de Boccaccio. Al mismo tiempo, la elección de las obras tampoco constituye un tema banal. El estudio propuesto ha sido bastante meditado previamente con la intención de poder establecer alguna relación significativa entre ellas. Por tanto, no es casualidad que ambas obras pertenecieran a un mismo periodo artístico y tampoco que la figura protagonista fuera femenina en ambos casos. Este estudio no solo va a analizar el simbolismo vegetal, sino la propia 
figura femenina integrada en el prototipo de femme fatale. Solo así podrá entenderse la representación de dicha flor en ambas escenas.

\section{Objetivos}

Partiendo de algunas obras del artista británico John William Waterhouse, el objetivo principal de este estudio consiste en comprobar la relación establecida entre el modelo de femme fatale propuesto por los prerrafaelitas y el simbolismo vegetal que se esconde en sus obras. Para ello, el análisis se centra en dos composiciones del artista -Ofelia e Isabella and the Pot of Basil-, pues en ellas se observan tanto las características del nuevo prototipo artístico como la influencia que ejercía el entorno natural en la historia de estos dos personajes femeninos. A su vez, para estudiar la relación comprendida entre ambos, resulta imprescindible tratar determinados aspectos teóricos de manera previa, como la amapola o la adormidera, dejando a un lado la cuestión de la femme fatale tan vista por los investigadores en estudios anteriores. La preocupación por este tema se debe al escaso conocimiento en lo que a botánica se refiere, un conocimiento vital a la hora de establecer un análisis de semejantes características. De esta manera, este estudio se desarrolla en torno a dos aspectos o puntos principales: la descripción detallada de la amapola o adormidera por un lado, y la conexión que se genera con respecto al estereotipo de femme fatale por otro. El seguimiento de esta estructura es el que va a ayudar a cumplir con los objetivos específicos propuestos, los cuales se resumen en:

- El estudio de la amapola o adormidera desde una perspectiva distinta a la acostumbrada - centrada esta vez en el simbolismo-, de forma que se muestran con ello todas las posibilidades que un elemento natural como este ofrece.

- El análisis de la pintura de Waterhouse a partir de dos de sus obras para observar las similitudes existentes entre ambas, pero también la importancia que el autor decide otorgarle a la naturaleza - lo cual se observa a lo largo de su carrera artística.

\section{Material y método}

El método a seguir en el desarrollo de este estudio es el iconográfico-iconológico. Bien es sabido que de todas las opciones posibles para el análisis de las obras de arte, este método es uno de los más recurrentes por los investigadores tanto por su globalización como por su eficacia dentro del estudio de la Historia del Arte. La descripción de las imágenes y la interpretación posterior es la que 
mejor va a ayudar a esclarecer el simbolismo de la amapola y, por tanto, el significado de ambas obras.

En cuanto a las fuentes requeridas, este estudio centra su atención en distintos materiales especializados en botánica, no solo para conocer los componentes de la amapola o adormidera, sino también para hacernos conscientes de la historia que esconde y que puede llegar a ser la responsable directa de su significado en las obras de Waterhouse. En este sentido, el libro Plantas y árboles en el mundo clásico, de Prieto Esteban, puede considerarse un buen punto de partida para adquirir una visión general sobre el mundo vegetal, así como el artículo de López Terrada centrado en la mitología clásica y el simbolismo de estos elementos naturales. Ya para dirigir la atención hacia la amapola o adormidera, los artículos de Becerra Romero y Gómez Fernández — «La adormidera en el Mediterráneo oriental: planta sagrada, planta profana» y «Amapola, efímera flor: las mil y una caras de la amapola silvestre», respectivamentepueden resultar de gran ayuda.

De la misma forma, la consulta a clásicos como la tragedia de Shakespeare y los poemas de Keats se considera imprescindible, ya que sus líneas y el modo de expresión de los autores pueden decir bastante en relación con la mentalidad que tenía el ser humano sobre la figura femenina. También resulta digno de mención el manual de Cavallero - J. W. Waterhouse and the Magic of Color-, aunque un análisis de estas características requiere de materiales más especializados en las figuras a tratar. Por ello conviene mencionar algunos documentos como la tesis doctoral de Capona Pérez sobre Ofelia -Ofelia o el mal imaginario. Estudio de la evolución del personaje de Ofelia en tres obras dramáticas desde una mirada de género- o el artículo de Garrosa Gude sobre Isabella - «Lisabetta y el tiesto de albahaca (Decamerón IV, 5): el sustrato folclórico de Boccaccio».

\section{Amapola roja o adormidera}

Según el diccionario de la Real Academia Española de la Lengua existen notables diferencias entre la amapola y adormidera. Independientemente de que ambas pertenezcan a la misma familia de papaveráceas y muestren una imagen similar, hay muchos elementos que no coinciden entre ellas. El más destacado se encuentra relacionado con el espacio donde se instala cada una, ya que la amapola se caracteriza por mostrar una imagen asilvestrada y totalmente libre, de modo que su campo de actuación se limita a los sembrados, los cuales logra infestar con suma facilidad en un corto periodo de tiempo. La adormidera, por su parte, suele cultivarse y no todos los países permiten su producción, pues la semilla que permanece en su interior dispone de una cápsula o cabeza con la que 
el ser humano puede extraer opio y que la convierte, al mismo tiempo, en una planta apreciada o rechazada según época y cultura.

Científicamente, ambas pertenecen a la familia de las papaveráceas y se identifican como Papaver. Dentro de esta familia existen además otras clases distintas a la Papaver rhoeas L. y la Papaver somniferum L., que muestran ciertas características comunes relacionadas con "el jugo o látex de color blanquecino, amarillento e incluso incoloro, que desprenden cuando se cortan o se dañan» (Becerra, 2006: 7), así como la gran variedad de componentes o sustancias conocidos como alcaloides. Estos responden a una serie de compuestos nitrogenados característicos de los vegetales que dotan a la flor de propiedades notables. En este caso, el compuesto orgánico principal en las adormideras es la morfina, mientras que el de las amapolas rojas es la readina. A pesar del efecto narcótico que ambas presentan, la amapola roja no es tan peligrosa. Ello es debido a su baja proporción de morfina y a la falta de determinadas "propiedades tóxicas y enteógenas que [contiene] su hermana mayor la adormidera» (Gómez, 2008: 42-43).

Ambas especies no solo presentan una simbología particular en el arte al ser las más conocidas dentro de la familia de las papaveráceas. Su historia es también reseñable en este punto, ya que se han encontrado evidencias arqueológicas sobre dichas especies desde tiempos remotos. No hay duda de que los europeos en el pasado ya sabían de su existencia. Tampoco se discute la gran aceptación que tuvieron en la península Ibérica durante el Neolítico, aunque otros espacios europeos como Francia, Alemania o la antigua Yugoslavia también disfrutaron de su presencia, de forma que se abarca una cronología extensa que llegaba incluso hasta época romana. Aun así, el verdadero origen de estas especies se encuentra en el Próximo Oriente gracias a las excavaciones arqueológicas realizadas por la zona. Los restos hallados donde se contemplan distintas representaciones artísticas sobre la adormidera, pertenecen a la antigua Mesopotamia. Egipto también mostraba representaciones pictóricas sobre la amapola en tumbas, pero la adormidera no formaba parte de su repertorio hasta la etapa ptolemaica, momento en que empezó a ser cultivada en tierras cercanas al Nilo (Becerra, 2006: 10). Lo que parece estar claro es la difusión que experimentaron ambas especies hacia el Mediterráneo oriental. Su expansión pudo ser producto de «las exportaciones de cereales que comenzaron hace unos ocho mil años con la [...] naciente cultura neolítica» (López, 2006: 28). Estas exportaciones fueron las que terminaron propagando la función medicinal y comestible de ambas. En el caso de la amapola o "flor de fuego», la mezcla de ingredientes permitía elaborar preparados infantiles con los que dormir a los niños gracias al efecto narcótico que había en sus flores (Gómez, 2008: 43). Como remedio antiguo, las 
propiedades sedantes de esta ayudaban a provocar el sueño, pero también a calmar los accesos de tos. La adormidera, por su parte, interesó desde un punto de vista farmacológico ya que contenía opio, el cual se extraía desecando el jugo lechoso de las cápsulas (Prieto, 2011: 121-122).

No obstante, uno de los aspectos más reseñables se encuentra en las fuentes clásicas, pues estas sirven muchas veces como inspiración para la elaboración de determinadas obras de arte, especialmente aquellas desarrolladas a partir del Renacimiento. El mundo griego, así como el romano, siempre han estado considerados los dos máximos representantes de la mitología antigua. En este sentido, los autores clásicos de ambas civilizaciones mostraban un gran apego por las divinidades mitológicas y todos los aspectos relacionados con este tema, de modo que cuando hablaron de la amapola y la adormidera, no tardaron en vincular ambos elementos a la mitología. Teniendo en cuenta que la adormidera se difundió gracias a las exportaciones de cereal, los autores terminaron asociándola con la diosa Deméter, es decir, la diosa de la agricultura y, por ende, del cereal. A simple vista, esta podría ser la interpretación más correcta, pero no hay que olvidar que la mentalidad del ser humano en la Antigüedad no era la misma que en la actualidad. En el mundo griego y romano, las cuestiones mitológicas tenían un gran peso en la sociedad y se apoyaban constantemente en sus historias. Por tanto, el hecho de que la adormidera se vinculase con Deméter se debe a su triste historia. Las propiedades de esta planta, capaces de provocar sueño y olvido, eran las idóneas para poder aliviar su pena tras el rapto de hija Perséfone por el dios del Inframundo (Becerra, 2006: 13). Según Pausanias, fue la desesperación la que llevó a Deméter a masticar la planta; mientras que Ovidio decidió introducir a Lete en la narración -el río infernal del Olvido, según la mitología griega-, que fue quien terminó conduciendo a la diosa a una caverna donde florecía una multitud de adormideras, con las que poder aletargarse.

Al igual que la adormidera, la amapola silvestre se vincula con el culto de una diosa de la mitología. La diosa escogida es Afrodita y a ella le acompañan otras vinculadas al tema amoroso. A diferencia de la anterior, utilizada para aliviar las penas o al menos para olvidarlas, la "flor de fuego" estaba presente en momentos de alegría, como bodas u otras celebraciones. Según Ovidio, esta planta mostraba una serie de componentes que daban como resultado una bebida afrodisíaca que solía servir en la noche de bodas para desposar a la mujer. Por tanto, es importante distinguir ambas clases del mismo modo que lo hacían los antiguos (Prieto, 2011: 122). 
La mujer como tema o símbolo es un elemento que, en época prerrafaelita, llegó a tomar un fuerte protagonismo en los diferentes campos de pintura, literatura, poesía y música. En poco tiempo, las mujeres pasaron de estar prácticamente en la sombra a convertirse en protagonistas. La fascinación por estas en el arte, unida al orden social establecido, hizo que determinados artistas de la hermandad como Rossetti, Millais o Hunt las tomasen como modelos, de modo que se integró su figura dentro del panorama artístico y se difundió una imagen negativa debido a la doble moral de la sociedad y a todos los cambios que estaban produciéndose a favor de las mujeres. Algunos de los más significativos y difundidos en las artes señaladas se relacionan con la sexualidad femenina, la cual no se limita a la satisfacción del marido en el lecho conyugal, sino que trasciende a otros ámbitos. En este sentido, se describen numerosos ejemplos de matrimonios desgraciados donde la mujer se ha lanzado al adulterio sin pensarlo (Caparrós, 1999: 40). También resulta frecuente mostrar la perturbación y el desasosiego que causa en esta el amor no correspondido, así como otros aspectos relacionados con la crueldad, la traición, la venganza, la perversión, la seducción, etc., que hacen de la mujer un "ser maligno». Cierto es que esta manera de pensar siempre ha estado presente en el ser humano, aunque ha sido la concepción decimonónica del mundo en el campo artístico y literario la que ha terminado desarrollando el estereotipo de femme fatale (Eetessam, 2009: 234). Todo ello se muestra en las dos obras señaladas, aunque es el estudio simultáneo de los elementos vegetales representados en las imágenes el que ayuda a comprender el significado de la obra.

\section{La amapola y su interpretación en Ofelia (1894)}

La segunda versión de Ofelia elaborada por el artista en 1894 representa el momento previo a la muerte de uno de los personajes secundarios de Hamlet - una de las tragedias más conocidas de William Shakespeare. La narración de este autor no termina de dejar claro si esta cae por accidente al arroyo u opta por suicidarse sumida por la desesperación y la locura que le produce el amor no correspondido de Hamlet. La teoría más recurrida suele ser la segunda, ya que le proporciona a la obra más dramatismo por el fatal desenlace de la joven. Algunos autores aseguran que el suicidio es la única salida que le queda cuando la locura se apodera de ella. Al mismo tiempo, este acto es el elemento que la independiza de la obra de Shakespeare hasta convertirse en un «símbolo del suicidio femenino» (Rodríguez, 2007: 26). En este caso, la locura o el desorden psicológico de Ofelia pasó a convertirse, dentro del arte 
prerrafaelita y la literatura británica del momento, en el modelo de todas aquellas mujeres que habían sido golpeadas por el amor. Muchas de ellas vieron en Ofelia una figura atractiva, aunque perjudicial por el fatal desenlace.

A pesar de la ingenuidad, bondad y fidelidad que tanto caracteriza a la doncella, esta es reconocida como una de las figuras más representativas de la "fatalidad femenina». Por tanto, no hace falta ser rebelde para pertenecer a dicha categoría. Muchas veces la obediencia, así como otros de sus rasgos, son suficientes para determinar ese aura de fatalidad, pues esta se basa en la falta de control de la propia identidad que se produce en el momento en que muere su padre y es rechazada por su amado, es decir, cuando pierde todo referente masculino (Capona, 2004: 67-68). Ello la lleva a encontrarse en un escenario solitario, que suele identificarse con un paraje natural. Se podría decir que es la situación de desgarro y perturbación que sufre la que termina conectándola con la naturaleza. Las flores seleccionadas en la obra de Waterhouse no responden a ningún elemento aleatorio, sino que esconden un fuerte simbolismo que determina el propio significado de la composición. En el caso de Millais, la relación con lo efímero y lo caduco de las flores queda establecida al esparcir distintas especies sobre la Ofelia acuática, algo que en Waterhouse no resulta tan evidente a simple vista. Ello no quiere decir que el simbolismo vegetal no se produzca en esta obra, ya que si se analiza detenidamente y se compara con la obra de Millais, se puede observar todo lo contrario.

El lienzo de 1851 realizado por este otro artista, representa una serie de elementos vegetales con significado propio. Algunos investigadores observan «nomeolvides, ulmarias, ortigas, margaritas, narcisos, coronas imperiales, lirios, adonis, dedos de muerto... incorporados no como aderezos pueriles, sino como metáforas tanto de los defectos de Hamlet como de los sentimientos taciturnos de Ofelia» (Rodríguez, 2007: 30). Por otra parte, la presencia de amapolas también se hace notable alrededor de su figura gracias a ese intenso color rojo que alude al adormecimiento y al fenómeno de la muerte: dos conceptos similares en la mayoría de los casos. Principalmente, ambos son los dos significados más conocidos que adquiere la amapola dentro del ámbito artístico. En relación con esto, algunas veces la escena no precisa de este tipo de elementos para interpretarse correctamente, por lo tanto, las flores aparecen como un componente más. En cambio, otras veces, se requiere de su presencia para comprender o complementar el significado de la composición.

Si se desconoce el fatal destino de este personaje, difícilmente se puede llegar a imaginar que Ofelia está a punto de recurrir al suicidio. Sin embargo, un conocimiento amplio en simbolismo vegetal puede descifrar el significado de la pintura de Waterhouse. 
En este caso, la aparición de la amapola ensortijada entre el cabello de la joven le aporta un mayor dramatismo a la obra, ya que envuelve al personaje en una de las etapas más terribles de su vida. El encerramiento o encapsulamiento que el artista propone al coronarla con la flor carga a la imagen de un fuerte patetismo, más intenso que cualquier representación anterior. A diferencia de Millais, que representa la calma tras comprender que el sufrimiento de Ofelia por fin ha cesado (Cavallero, 2017: 77), Waterhouse proyecta ese estado de enajenación y perturbación al cual es sometida momentos antes del suicidio. Su primera versión sobre este personaje en 1889 no captó ese aura de locura a la cual estaba siendo sometida; sin embargo, se percibe una intranquilidad en el ambiente promovida por la intensa mirada de la joven hacia el espectador; una mirada que parece estar informando de algo. Aun así, es en su segunda versión cuando se descubre lo que está a punto de producirse gracias al posicionamiento de la amapola en la obra. La amapola, una flor que evoca al sueño y alude a la propia muerte, es representada en la cabellera de Ofelia, quien aparece acariciándose el pelo mientras toca sutilmente los pétalos con los dedos. Con esta excelente representación por parte de Waterhouse, el artista transmite las verdaderas intenciones de la joven, pues con ello parece haberse asumido la teoría del suicidio. Aparte, el hecho de que se represente en la cabeza de Ofelia evidencia el pensamiento suicida por parte de esta y confirma la teoría de que el artista británico también era partidario de su suicidio, de lo contrario, no representaría la amapola en este punto y más sabiendo la simbología que genera este tipo de elementos vegetales. Es cierto que la obra alude a una guirnalda de flores que Ofelia deposita finalmente sobre su cabeza, aun así llama la atención que de entre todas ellas, sea la amapola la protagonista.

En la última versión que el artista propone, vuelve a presentar a una joven desesperada, con la mirada perdida y sumida en un estado de enajenación en el que apenas es consciente de sus actos. Lo llamativo aquí vuelve a ser el protagonismo que toma la amapola en la obra, la cual vuelve a estar presente en la cabeza de esta, pero también en los ropajes. Parece ser que en su estado de locura, Ofelia se toma bastante en serio ese proceso de adornarse con las flores, pues en ellas parece encontrar a sus únicas compañeras durante los duros momentos de perdición que está sufriendo al ser traicionada por la humanidad entera (Cavallero, 2017: 77). Por este motivo, Waterhouse recurre a la amapola en diversas ocasiones. En ella observa una vía de escape, en este caso, la muerte. Solo ella la puede salvar del sufrimiento padecido. 
Nuevamente, el artista vuelca su atención hacia la literatura representando uno de los poemas más conocidos del escritor británico John Keats. El poema, elaborado en 1820, fue inspirado en el Decamerón de Giovanni Boccaccio. En esta ocasión, el escrito, compuesto a mediados del siglo XIV, se dispuso en diversas jornadas con el fin de agrupar distintos bloques temáticos. En lo que respecta a Lisabetta y Lorenzo, es decir, la historia en la que se inspiró John Keats más tarde, esta aparece en la IV jornada dedicada a la narración de amores fallidos cuyos desenlaces resultaban desgraciados (Garrosa, 2010: 164). Gracias a este tipo de historias logran percibirse las grandes dotes narrativas del autor, pues no muchos pueden enlazar determinados temas, como el amor, la venganza y la muerte, con la misma facilidad que lo hace Boccaccio. De hecho, esta podría ser una de las razones que motivaron a Keats a recoger tal relato, adaptándolo a su gusto y llevando a cabo determinados cambios relacionados con el lugar donde transcurre la acción y el nombre de la protagonista. En este sentido, el escenario de la historia pasa de Messina a Florencia, mientras que la protagonista ya no responde al nombre de Lisabetta sino al de Isabella.

Por otra parte, cabe tener en cuenta que esta historia no solo tuvo repercusión en el ámbito literario, también fue importante dentro del artístico. El poema Isabella or the Pot of Basil, de Keats, terminó siendo trasladado a la pintura gracias a algunos de los integrantes de la Hermandad Prerrafaelita que decidieron plasmar su historia en el lienzo, y así convirtieron su representación en uno de los proyectos más enriquecedores de la hermandad al implicar el primer núcleo prerrafaelita compuesto por Rossetti, Hunt y Millais (Hilton, 2000: 35). Tal fue la repercusión que Waterhouse, siguiendo los mismos parámetros que los grandes precursores de este arte británico, dedicó parte de su producción artística a dicho pasaje, y elaboró una versión al respecto. La escena escogida por este es quizás una de las más desgarradoras por el sufrimiento que se percibe en la joven mientras abraza la maceta de albahaca donde ha sido depositada la cabeza de su amado. Al mismo tiempo, se convierte en una escena macabra no solo porque conserva la cabeza en una olla, sino porque ha sido ella misma la que ha depositado sus restos en dicho recipiente. Así pues, lo que puede parecer una imagen triste y romántica guarda en realidad un humor bastante oscuro y macabro. Resulta repugnante pensar que la joven le está llorando a una cabeza que ella misma ha enterrado y que poco a poco se va pudriendo en el interior de una olla, mientras su cabello va mezclándose con las raíces de la albahaca.

Esta acción es uno de los aspectos fundamentales que conectan al personaje de Isabella con el estereotipo de femme fatale, aunque 
no el único. Algunos incluso señalan que el propio amor que siente

hacia Lorenzo ha sido el que lo ha condenado a ese destino. Probablemente si esta no hubiese desobedecido a sus hermanos y no hubiese aceptado dicha relación clandestina desde un principio, Lorenzo seguiría vivo. Afirmaciones como estas son las que evidencian el poder patriarcal que todavía se ejerce sobre la mujer, ya que se busca a toda costa la forma de culpabilizar al sexo femenino de las desgracias del hombre cuando, en realidad, ambos consienten la relación desde un principio. Muchas veces, centrándose en ese aspecto, se olvidan de que fueron sus hermanos quienes acabaron con la felicidad de los amantes. Sin embargo, es la desobediencia de Isabella para con ellos la que destaca sobre el acto cruel e imperdonable de estos, y potencia así una imagen femenina rebelde incapaz de acatar las órdenes del varón. Es precisamente esta imagen amenazante de mujer la que proyectan los prerrafaelitas en sus lienzos gracias a la recreación de la femme fatale (Caparrós, 1999: 41).

Concretamente, Hunt y Waterhouse son quienes mejor representan este estereotipo tanto por la recreación de algunos de los rasgos físicos más característicos de estas mujeres como por el estado en que parecen encontrarse. En estos casos, la mirada juega un papel fundamental al reflejar, en el caso de Hunt, la locura femenina. A diferencia de este, Waterhouse plasma esta misma sensación con los ojos cerrados. Parece ser que el artista concibe mejor ese estado de locura sin representar la mirada perdida o loca de la joven. Ya lo hizo con Ofelia en 1894 y lo vuelve a hacer con Isabella años después.

Realmente, la elección de ambas composiciones sobre Waterhouse para este trabajo no tiene que ver exclusivamente con la recreación de la amapola en ellas, sino también con todas las similitudes que comparten. En primer lugar, el estado de demencia o delirio que reside en ambas protagonistas, es plasmado por el artista de la misma forma. Tanto en Ofelia como en Isabella, Waterhouse representa a dos jóvenes con los ojos cerrados y la cabeza erguida mientras la locura se va apoderando de ellas, y las consume poco a poco hasta llevarlas a la muerte. En segundo lugar, la naturaleza queda inmediatamente conectada con esa figura estereotipada de la mujer tal y como se ha visto en el ejemplo anterior.

Más allá de las alusiones constantes a la naturaleza en ambos textos, Waterhouse se centra en ella por la simbología que esconde. El poema no habla en ningún momento de amapolas o adormideras, en cambio sí menciona rosas, jacintos, lirios y otros elementos característicos como riachuelos o bosques. Hace también referencia a las estaciones del año y a algunos fenómenos naturales como el rocío, mientras que la amapola no aparece por ninguna parte. No obstante, llama la atención la metáfora que el poeta establece sobre 
las rosas, por lo que tendría más sentido plasmar en el lienzo esta

flor y no otra. Aun así, su simbología le concede el papel protagonista en la obra. Parece ser que Waterhouse creía firmemente en estos temas debido al elevado número de representaciones creadas donde la naturaleza ejercía un papel relevante dentro de la propia composición. De hecho, podría ser este elemento el que le permitía crear numerosas representaciones de femmes fatales envueltas todas ellas sobre un halo de leyenda.

Tal vez la intención del artista fuese mostrar esa doble cara que los escritos y la mayoría de obras plásticas trataban de ocultar, de modo que la representación de las rosas en el lienzo únicamente reflejaría aquella visión triste, pero a la vez dulce, que solía tenerse sobre el relato. A través de su obra y el papel que toma la amapola en ella, se vislumbra la intención del autor, pues todas las interpretaciones generadas sobre esta flor en el lienzo seleccionado adquieren un carácter negativo que lo diferencian del resto. Dicho carácter alude a la muerte, aunque existen múltiples teorías. Las dudas surgen, principalmente, al tratar de relacionar este elemento vegetal con alguno de los personajes, ya que la muerte termina actuando sobre los dos protagonistas indistintamente. La diferencia reside en el modo en que actúa sobre ellos: mientras Lorenzo es asesinado, Isabella muere de pena. En este caso, la amapola podría interpretarse de tres formas distintas atendiendo al elemento temporal, es decir, si la flor se ve desde una perspectiva pasada, presente o futura. Esta condición influye en la interpretación posterior. Pero ¿qué tiene que ver el tiempo en todo esto? El tiempo es en realidad el factor que determina si la planta se refiere a la muerte de Lorenzo o a la de Isabella. Si es la muerte de Lorenzo, la amapola pasaría a ser una confirmación de lo que ya de por sí es un hecho. La muerte del amante o, mejor dicho, el asesinato de este en manos de los hermanos de la joven ya ha tenido lugar semanas atrás. La representación de la amapola podría entenderse aquí como un respaldo al resto de elementos que reflejan de un modo $u$ otro ese fatal acontecimiento. Por un lado, la aparición de la maceta u olla de albahaca en el margen izquierdo de la composición, es algo que en principio no necesitaría ningún tipo de respaldo, ya que en su interior se halla enterrada la cabeza del amante. Sin embargo, todo aquel que desconozca la historia puede pensar que la muerte se oculta en esta imagen al observar la amapola en un primer plano. Por otro lado, esta podría relacionarse también con la calavera que aparece representada en el pedestal donde se postra la maceta. A simple vista, puede parecer que la recreación de estos dos elementos no necesite de ningún respaldo. A pesar de ello, no hay que olvidar que Lorenzo fue enterrado por los hermanos de Isabella en el bosque y su muerte se produjo en ese mismo lugar, de modo que esto sería como 
recordar de alguna manera, el vínculo que este personaje mantiene con la naturaleza.

La segunda interpretación relacionaría la amapola con un futuro próximo. En base a ello, esta pasaría a asociarse con el personaje femenino, y aludiría a la muerte de Isabella. Igual que en las distintas versiones sobre Ofelia, el artista está aventurando al espectador a ese fatal desenlace que está a punto de producirse. La joven, sumida en una profunda pena y abatida por la desolación, está llegando a sus últimos días de vida. El episodio representado aquí es prácticamente el anterior a su muerte, puesto que todavía posee su preciada maceta. Se podría decir que es el robo de esta el broche final a su historia, en definitiva, lo que la acaba condenando a la muerte. La desaparición de la maceta y con ella, los restos de su amado, hace que Isabella no tenga nada más por lo que vivir, de modo que termina muriendo trágicamente, apenándose por la ausencia total de su amado hasta exhalar su último aliento de vida. Dicho esto, la amapola no dejaría de ser un guiño a ese futuro inmediato que está a punto de sufrir todavía sin saberlo; de ahí la proximidad de la amapola hacia su propia figura y probablemente el hecho de que haya sido representada a su espalda.

Finalmente, la presencia de este elemento vegetal en la imagen podría relacionarse también con ese momento exacto del relato. En él se muestra a una Isabella ida, completamente sumida en un profundo estado melancólico que la hace no ser consciente de donde se encuentra. Se ha olvidado de todo aquello que la rodea, incluso de vivir, de ahí que pueda tener cabida ese paralelismo entre el personaje de Isabella y la muerte en este punto del relato. Realmente, la melancolía es un estado anímico bastante similar en algunos aspectos a la propia muerte, ya que presenta determinados rasgos que se acercan más a esta que a la propia vida. Por ello, el personaje de la enamorada se muestra de una forma casi fantasmal, como si estuviese «muerta en vida». Según Mínguez y Rodríguez (2014: 371):

La melancolía es un estado anímico o temperamento que médicamente se ha caracterizado por mostrar síntomas de locura, a veces alternado con episodios maníacos, además de depresión y momentos de ansiedad. Es un estado de indecisión, que comienza como una enfermedad mental, aprisionando al hombre afligido [o a la mujer en este caso] en círculos de cuestiones sin solución. En todos los casos el efecto es el mismo: depresión, acompañado de miedo, un estado interior de silencio, y una percepción del mundo difusa, en un proceso de pérdida de sentido.

Fuera de la cuestión temporal existe otra alternativa, quizás más válida, al ser la única que conecta directamente la muerte con el sueño, que es, en definitiva, el aspecto fundamental que caracteriza 
a esta flor. Esta interpretación quedaría fuera de la clasificación anterior al tratarse de un sueño revelador que tiene la joven tras la ausencia de su amado, el cual le permite descubrir el lugar donde se halla enterrado el cuerpo del difunto. $Y$ esta teoría termina convirtiéndose en la más potente de todas por distintas razones. En primer lugar, las sustancias que componen la amapola permiten aletargar a una persona, de modo que tendría sentido que se representara en el primer plano de la obra, pues estaría haciendo referencia a ese sueño revelador de la joven. En segundo lugar, los clásicos ayudan a justificar todavía más esta teoría mediante afirmaciones como la siguiente: «entre tanto de amapola ceñida la plácida frente viene noche y consigo trae los negros sueños» (Prieto, 2011: 121). En esta ocasión, Ovidio se refiere a la amapola de una forma negativa, de lo contrario no hablaría de "negros sueños», sino de sueños sin más. Esto es lo que permite realmente vincular la presencia de la amapola con el sueño de la joven, ya que su visión no es para nada agradable. En este sentido, estaría más relacionada con un mal sueño. Finalmente, el propio Keats hace un guiño casi sin darse cuenta a ambos aspectos cuando dice lo siguiente: «and she had died in drowsy ignorance» (Keats, 1990: 194).

Con todo lo dicho en esta última parte, resulta evidente la relación intrínseca que el sueño y la muerte presentan, así como la importancia de recrear la amapola en el lienzo, al ser esta flor la que conecta ambos conceptos.

\section{Discusión y conclusiones}

Con el presente trabajo se ha pretendido mostrar la relación existente entre el estereotipo de femme fatale acuñado por los prerrafaelitas y la naturaleza representada en los lienzos de Waterhouse. Gracias al análisis de dos de sus obras, se ha comprobado la conexión habida entre ambos aspectos. Por un lado, el apartado introductorio sobre la flor ha sido esencial para desarrollar el trabajo. La comprensión de sus características y el uso que se le ha dado a lo largo de la historia, han ayudado a interpretar, de la manera más coherente posible, su aparición en cada una de las obras seleccionadas. Por otro lado, el análisis establecido en el segundo apartado ha servido para observar qué otros significados presenta la amapola, más allá de la tradicional interpretación que asocia esta especie vegetal con el sueño. Se podría decir que el estudio realizado con anterioridad ha sido el que ha permitido relacionar la amapola con otra variante, en este caso la muerte, y se ha convertido en el concepto vertebrador de ambos personajes. Al mismo tiempo, se ha demostrado que un elemento tan simple como parecen ser las flores puede llegar a ofrecer información valiosa respecto al contexto en el que aparecen representadas y extraer, a 
su vez, reflexiones interesantes que pueden acercar cada vez más al individuo a la mentalidad de los artistas prerrafaelitas, concretamente, a la mentalidad de Waterhouse.

\section{Bibliografía}

Becerra Romero, Daniel. 2006. "La adormidera en el Mediterráneo oriental: planta sagrada, planta profana». Habis 37: 7-16.

Caparrós Masegosa, Lola. 1999. Prerrafaelismo, simbolismo y decadentismo en la pintura española de fin de siglo. Granada: Universidad de Granada.

Capona Pérez, Daniela. 2004. Ofelia o el mal imaginario. Estudio de la evolución del personaje de Ofelia en tres obras dramáticas desde una mirada de género. Tesis doctoral. Santiago de Chile. Universidad de Chile.

Cavallero, Dani. 2017. J. W. Waterhouse and the Magic of Color. Jefferson, NC: McFarland \& Company.

Eetessam Párraga, Golrokh. 2009. "Lilith en el arte decimonónico. Estudio del mito de la femme fatale». Revista Signa 18: 229-249.

Garrosa Gude, José Luis. 2010. "Lisabetta y el tiesto de albahaca (Decamerón IV, 5): el sustrato folclórico de Boccaccio». Cuadernos de Filología Italiana (volumen extraordinario 6): 163177.

Gómez Fernández, J. Ramón. 2008. «Amapola, efímera flor: las mil y una caras de la amapola silvestre». QEJ. Bricojardinería \& paisajismo: Revista profesional de distribución en horticultura ornamental y jardinería 166: 42-43.

Hilton, Timothy. 2000. Los prerrafaelitas. Barcelona: Destino.

Keats, John. 1990. John Keats, editado por Elizabeth Cook. Oxford: Oxford University Press.

López Terrada, María José. 2006. «El mundo vegetal en la mitología clásica y su representación artística». Ars longa: cuadernos de arte 14-15: 27-44.

Mínguez, Víctor e Inmaculada Rodríguez Moya. 2014. «Napoleón y el espejo de la Antigüedad: arqueología de las imágenes del poder». Ars longa: cuadernos de arte 24: 279-281.

Prieto Esteban, Ma Jesús. 2011. Plantas y árboles en el mundo clásico. Madrid: Áurea Clásicos.

Rodríguez Navarro, Ma Victoria. 2007. "Dos etapas en el último viaje de la triste Ofelia: Millais y Rimbaud». En Littérature, langages et 
arts: reconstres et création, coordinado por Dominique Bonnet et al., 26-38. Huelva: Universidad de Huelva.

Shakespeare, William. 1980. Hamlet, Macbeth. Acto IV, Escena VII, editado por José María Valverde. Barcelona: Planeta.

Soler Moratón, Melania. 2015. «Gustav Klimt y Lilith: la representación de la new woman como femme fatale». Conferencia presentada en el VII Congreso Virtual sobre Historia de las Mujeres. Jaén: Archivo Histórico Diocesano de Jaén, los días 15-31 de octubre de 2015. 


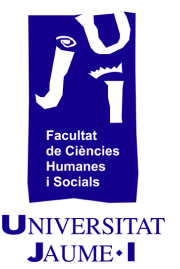

\section{El Torrelló del Boverot (Almassora),}

un jaciment clau per a comprendre l'organització del territori de la província de Castelló durant l'edat del bronze i l'edat del ferro 
I. Resum

En aquest treball tractarem l'evolució del jaciment del Torrelló del Boverot durant l'edat del bronze i l'edat del ferro i el compararem amb una selecció d'altres jaciments de la província de Castelló que tenen la mateixa cronologia. En primer lloc comentarem les característiques principals d'ambdues etapes històriques, incidirem en les cultures del bronze valencià i la cultura ibera, respectivament, i a continuació presentarem el Torrelló d'Almassora i, més breument, els altres jaciments que hem escollit de diverses localitats. En concret, presentarem els jaciments arqueològics de Vinarragell, la Torre d'Onda, el Torrelló d'Onda, l'Abric de les Cinc, el Tossal del Mortòrum, la Solivella i el Puig de la Nau.

Després d'exposar les característiques de les diferents èpoques, les de les cultures corresponents i quins són els llocs que tractem, es compararan els materials. Per a dur a terme la comparació, es tindrà en compte, a més de la cronologia, la similitud que tinguen els objectes que hem escollit del Torrelló amb els que hi ha a les altres poblacions anteriorment esmentades. Oferirem exemples concrets que confrontarem directament amb els més pareguts.

Tot això ens servirà per a conéixer i comprendre millor la història antiga de la província i per a posar en relleu la importància que va tenir el Torrelló del Boverot en el seu context històric, geogràfic $\mathrm{i}$ cultural.

Paraules clau: Torrelló del Boverot, edat del bronze, bronze valencià, edat del ferro, cultura ibera.

\section{Introducció i objectius}

Hi ha diversos jaciments a la província de Castelló que no s'han pogut excavar completament, l'extensió dels quals no s'ha pogut comprovar o no se sap si hi havia necròpolis per la mateixa zona o fins $i$ tot hi ha parts que han sigut destruïdes. L'objectiu per aquest treball ha sigut reunir la bibliografia sobre el Torrelló en un mateix document i comparar-lo amb altres jaciments, pareguts quant a cronologia i materials i de la mateixa província, per posar en relleu la importància que va tenir en l'antiguitat respecte a altres poblats $i$ també la influència com a punt de contacte amb el comerç mitjançant el riu Millars, en concret del comerç fenici. A més a més, es compararan les peces més emblemàtiques i representatives d'Almassora amb les més similars de les altres poblacions. 
La investigació per a aquest treball va començar en gener i es va allargar fins a octubre de 2018. L'inici va estar marcat pel començament de l'assignatura Treball Final de Grau, del grau d'Història i Patrimoni de la Universitat Jaume I. Durant aquests mesos, la investigació es va centrar en els nombrosos articles sobre els jaciments que hi ha a terres valencianes i en alguns llibres. També es va consultar en revistes dedicades principalment a les investigacions sobre les troballes en les excavacions de la província, com ara Quaderns de Prehistòria i arqueologia de Castelló, i altres que també inclouen apartats sobre aquesta zona. La recerca s'ha centrat en l'edat del bronze, les característiques del bronze valencià, l'edat del ferro i la cultura ibera al País Valencià i en el Torrelló del Boverot. A partir d'això s'ha buscat a més bibliografia sobre els jaciments o necròpolis de Castelló que coincidiren quant a cronologia i materials. Per tant, s'ha cercat informació general sobre les èpoques i cultures materials i també sobre exemples concrets.

Cal anomenar els dos autors principals en què ens hem basat: el principal investigador del Torrelló del Boverot, director del Museu d'Almassora dedicat al jaciment, Gerardo Clausell Cantavella. Ha escrit nombrosos articles amb la informació recollida a les excavacions, tant de la seua estructura com dels seus materials, els quals se citen sovint al llarg del treball. L'altre autor és Artur Oliver Foix, conservador del Museu de Belles Arts de Castelló. És un important investigador $\mathrm{i}$ referent en l'estudi sobre les èpoques $\mathrm{i}$ cultures que tractem, per la qual cosa és indispensable incloure el seu treball en la bibliografia utilitzada.

En el moment de començar aquest treball les publicacions sobre el Torrelló del Boverot s'havien aturat perquè no hi havia hagut excavacions durant setze anys i encara no hi havia cap article científic sobre les s'havien dut a terme a finals de l'any 2017. Sí que hi ha bibliografia d'altres excavacions d'urgència a la zona del Sitjar dels anys 2003 i 2005, però és breu i tampoc no es va tornar a intervenir l'àrea del jaciment, per la qual cosa no hi va haver més producció bibliogràfica. A causa d'aquests fets, gran part de la bibliografia trobada era molt antiga i ha calgut fer-ne una selecció per a no incloure teories desactualitzades, la qual cosa reduïa el nombre de les obres disponibles.

\section{El Torrelló del Boverot}

El jaciment del poblat està ubicat a l'extrem oest del terme municipal d'Almassora, a la província de Castelló, sobre un tell format a les terrasses superiors. Es troba a aproximadament $14 \mathrm{~km}$ de la mar al marge esquerre del riu Millars. La seua situació per 
damunt del riu i amb un bon domini del territori converteix aquest lloc en un punt amb una gran importància estratègica, no només davant de possibles invasions des de la mar, sinó també com a referent comercial de la zona costanera i com a comunicador amb I'interior, seguint el riu.

Així mateix, el jaciment del poblat ibèric del Torrelló del Boverot d'Almassora està format per les construccions extramurs del jaciment del Sitjar de Baix d'Onda. La construcció de la carretera CV10 i de dos polígons industrials en aquesta zona va afectar greument gran part del jaciment (Casabó, 2010: 61).

En estreta relació amb aquest poblat trobem una necròpoli al terme municipal d'Onda, a escassos 300 metres al nord-est del Torrelló d'Almassora. En la imatge següent podem observar la ubicació del Torrelló vora el riu Millars, així com de la necròpolis, a poca distància del poblat. Podem apreciar que el límit d'aquesta zona entre els termes municipals d'Almassora i d'Onda està marcat, així com el riu Millars, que separa de manera natural Almassora i Vilareal.

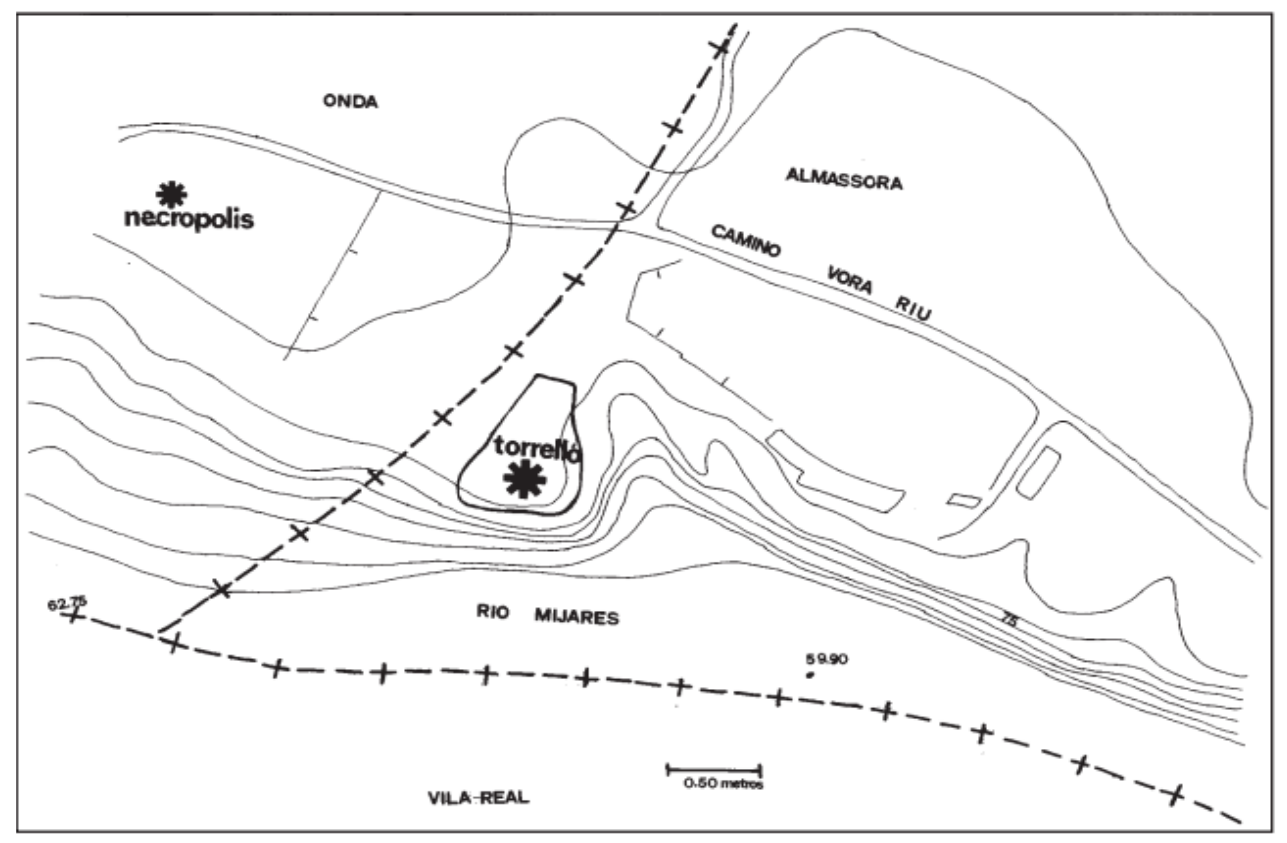

Figura 1. Mapa de la situació del Torrelló del Boverot i de la necròpolis, Gerardo Clausell Cantavella (1999: 116)

Al Torrelló del Boverot trobem majoritàriament estructures de l'edat del bronze i de l'edat del ferro. Dins d'aquesta última s'estudia la cultura ibera, així com el material que reflecteix la influència fenícia de l'època i també material propi de la cultura ibèrica, comentats en l'apartat següent d'aquest treball. Quant al bronze, $s^{\prime}$ han trobat habitacions dins del poblat.

Hem d'indicar que la cronologia de la necròpoli està estretament relacionada amb la del poblat, fet que queda patent en comparar les datacions dels materials trobats en cada àrea. Els moments 
d'assentament al Torrelló coincideixen amb els d'utilització de la necròpoli, així com es corresponen els períodes d'abandonament.

El jaciment del Sitjar de Baix posseeix una àmplia cronologia que va més enllà de la que tractem en aquest treball, per això ens centrarem en les fases que coincideixen amb les que ja hem comentat del Torrelló del Boverot.

\section{Estudi de la cultura material}

A continuació comentarem els materials d'alguns jaciments de la província de Castelló caracteritzats per tenir fases de l'edat del bronze i fases ibèriques amb exemples de material fenici gràcies a les relacions comercials que es van establir en aquesta zona. Aquests seran Vinarragell (Borriana), la Torre d'Onda (Borriana), el Torrelló d'Onda (Onda), l'Abric de les Cinc (Almenara), el tossal del Mortòrum (Cabanes), la necròpolos de la Solivella (Alcalà de Xivert). Distingirem segons el tipus de material, com per exemple ceràmica i bronze, i tindrem present també la seua utilització.

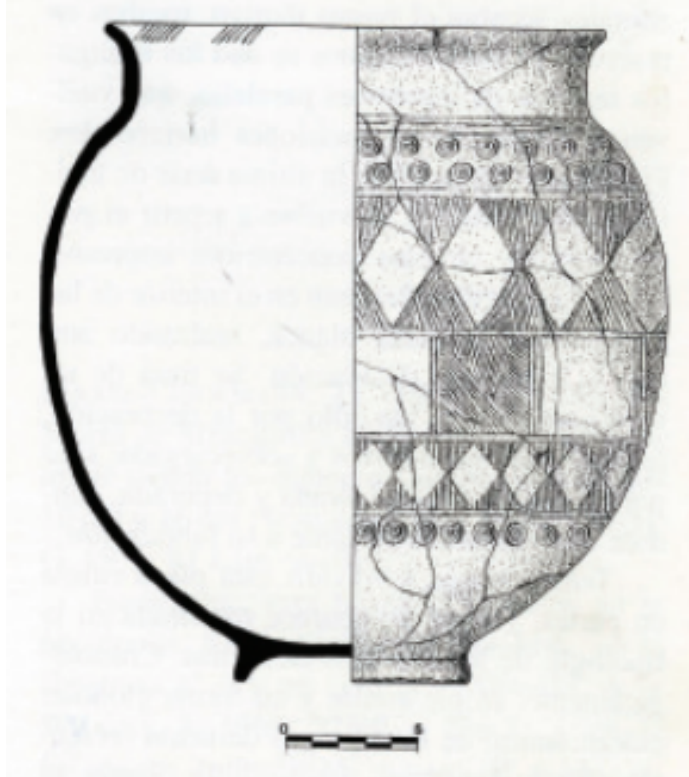

Figura 2. Gerard Clausell Cantavella, ceràmica decorada amb incisions i impressions, 1997

Al Torrelló d'Almassora trobem ceràmica de diversos tipus. Hi ha recipients que es van decorar amb motius geomètrics mitjançant incisions. Un d'aquests és l'atifell fet a mà amb forma globular i base anular recuperat a l'habitació 7. La decoració incisa geomètrica omple gran part de la superfície i repeteix les figures de la part superior a la inferior (figura 2). 


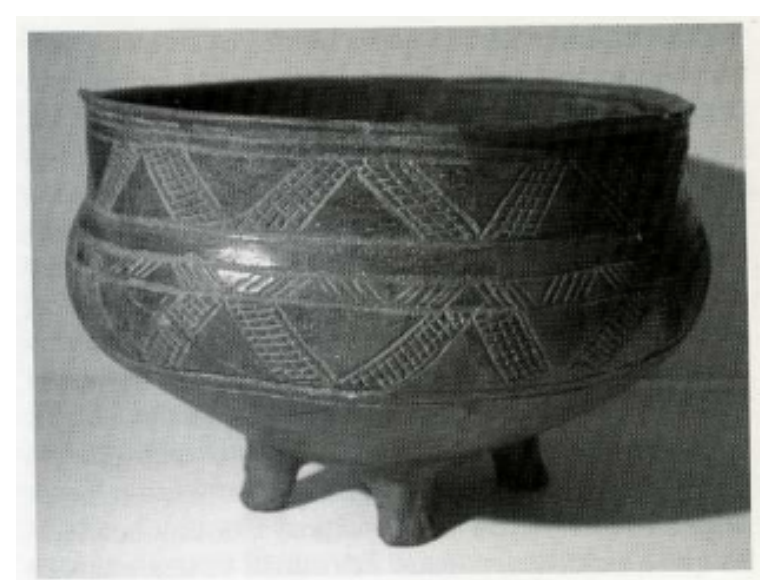

Figura 3. César Mateu, vas ceràmic trespeus, 2004

Destaca un vas ceràmic (figura 3) amb cos globular i la base convexa, ja que són les potes les que actuen com a suport. La decoració incisa de motius geomètrics es va fer abans de coure la ceràmica. L'anàlisi mineralògic va mostrar que el material amb què està elaborat no és autòcton i la cronologia és anterior a la de l'estança en què es va trobar, per tant segurament arribaria al Torrelló del Boverot per l'intercanvi comercial (Clausell, 2004: 174).

Al Tossal del Mortòrum es va trobar una peça similar (figura 4): un plat trespeus fenici, però sense decoració.

Aquesta forma en la ceràmica és comú als poblats fenicis o en aquells que hi han tingut contacte. Un exemple de decoració amb incisions del mateix jaciment està entre la ceràmica del bronze tardà (figura 5). Aquesta peça combina incisions que emmarquen el dibuix amb impressions que formen espigues (Aguilella, 2017: 70, 77).
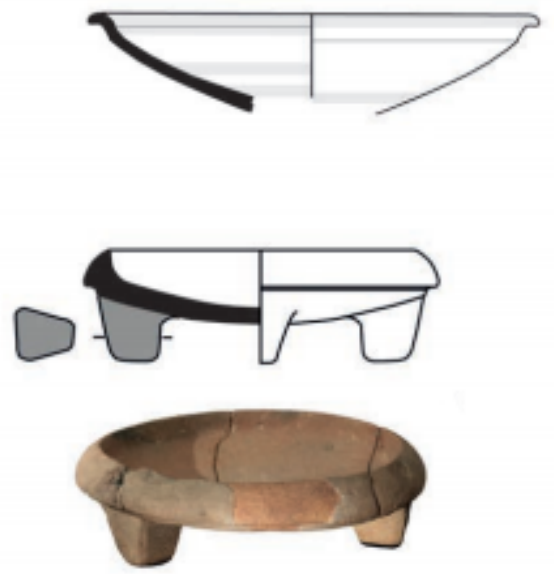

Figura 4. Gustau Aguilella, vas ceràmic trespeus del Tossal del Mortòrum, 2017 

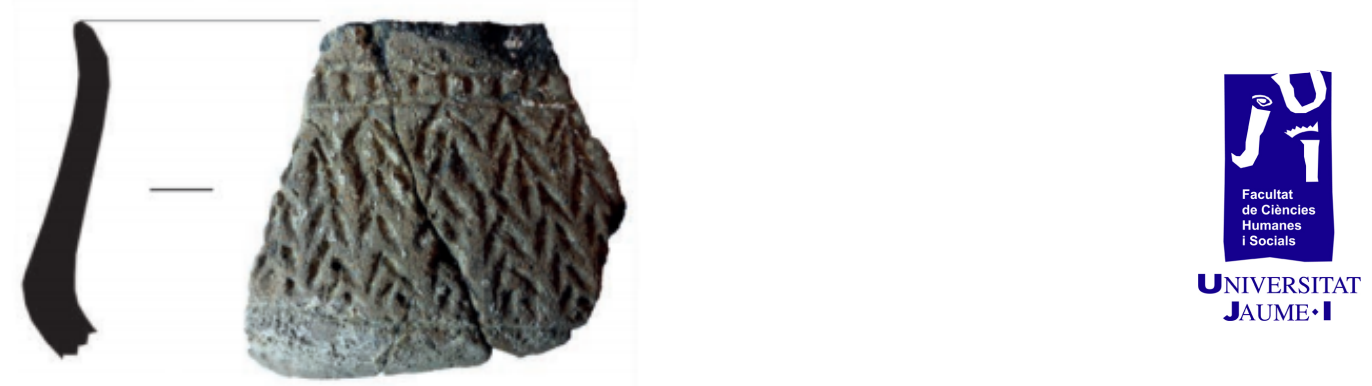

Figura 5. Gustau Aguilella, ceràmica del Tossal del Mortòrum decorada amb incisions, 2017

Un altre exemple de decoració feta amb incisions és la cassola brunyida feta a mà localitzada a l'habitació 5 , també amb decoració geomètrica però només a la part superior. És pareguda a una peça de Vinarragell (figura 6) (Clausell, 1997: 240-242). Durant la segona campanya d'excavacions de Vinarragell es van descobrir diversos tipus de ceràmica, entre els quals hi ha aquesta. Està feta a mà i té decoracions amb figures geomètriques elaborades mitjançant incisions (Mesado, 1974: 75, 103).
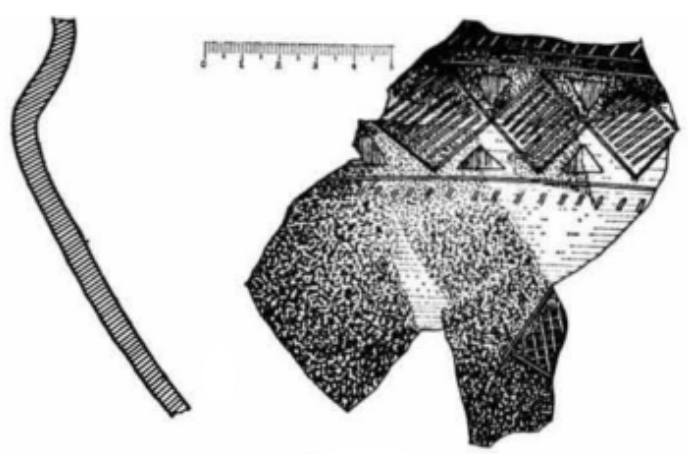

Figura 6. Norberto Mesado, ceràmica decorada amb incisions, 1974

Al Torrelló d'Onda també hi ha objectes amb incisions. Es tracta de ceràmica del bronze valencià, feta a mà, possiblement pensada per a l'àmbit domèstic. Malgrat la fragmentació de moltes de les peces, s'ha pogut establir que les formes més comunes dels atuells són la globular i l'ovoïdal (Gusi, 1974: 45).

Un altre tipus de decoració és la pintada. Trobem un exemple peculiar en un fragment de l'ibèric final que conté una escena festiva (figura 7). S'hi poden diferenciar dos personatges, un d'ells identificat com a femení que toca una flauta doble mentre que l'altre, masculí, balla. Estan representats de perfil, encara així és difícil identificar les parts de la cara d'ella, i les d'ell pareixen zoomòrfiques. Hi ha elements geomètrics al voltant que emmarquen el tema principal: a la dreta, una forma rectangular subdividida disposada verticalment amb un altre element amb traços més curts que es recolza sobre el primer, i a l'esquerra, una sèrie de rombes entre dues línies verticals (Clausell, 2000b: 93-94). 


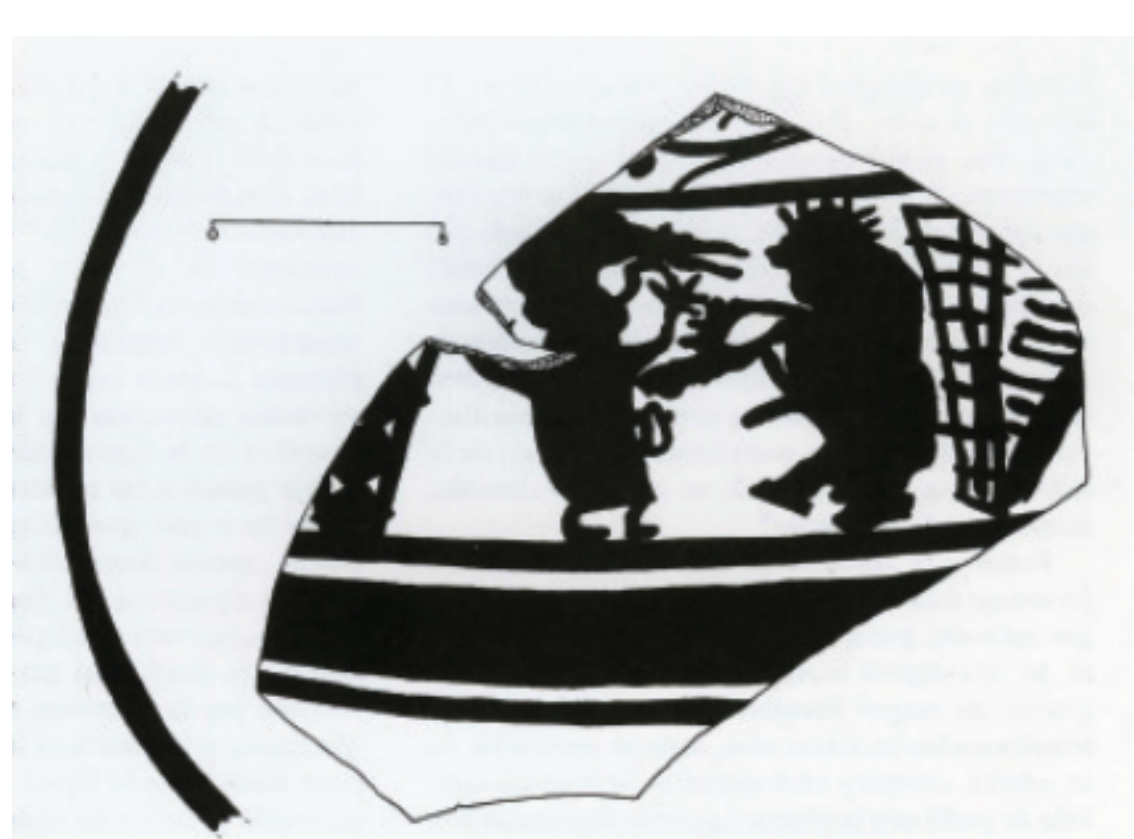

Figura 7. Gerardo Clausell Cantavella, vas iber amb escena de flautista i ballarí, 2000

Un altre exemple de ceràmica decorada amb pintura és el grup de ceràmiques ibèriques pintades del Torrelló d'Onda. Es van fer a torn i es van trobar molt fragmentades. En la major part dels casos eren dissenys geomètrics, encara que també n'hi havia algun zoomòrfic (Gusi, 1974: 50).

L'Abric de les Cinc també presenta peces pintades. Pertanyen a l'ibèric antic, la major part són fetes a torn, estan decorades amb diverses tonalitats de roig i formen figures geomètriques (Junyent, 1976: 77).

El Tossal del Mortòrum també ha aportat material amb pintura; és el cas de la pithos de cos ovoïdal i quatre anses dobles. Pertany al Ferro Antic, es va fer a torn i es va decorar amb pintura roja a l'exterior (Aguilella, 2017: 68, 72).

A més de decoracions amb motius geomètrics i vegetals també va aparéixer al Boverot un got de l'ibèric final amb una inscripció pintada per la part de fora. Es tracta d'un fragment de la peça original amb una inscripció incompleta causada per la fragmentació de la ceràmica (figura 8). El primer i l'últim signe estan incomplets, per la qual cosa només se'n pot proposar la possible identificació amb $r v$ o bé $a$ i $r$ o bé te respectivament. Els dos signes següents s'identifiquen amb claredat, són $b a$ i ti. El quart signe no està acabat d'escriure, però segurament es tracta de $r$. El cinqué torna a ser fàcilment recognoscible: és una $u$. Entre que acabem de comentar i l'últim que està incomplet hi ha escrit un signe de puntuació format per dos punts col-locats verticalment (Clausell, 2000b: 98). 


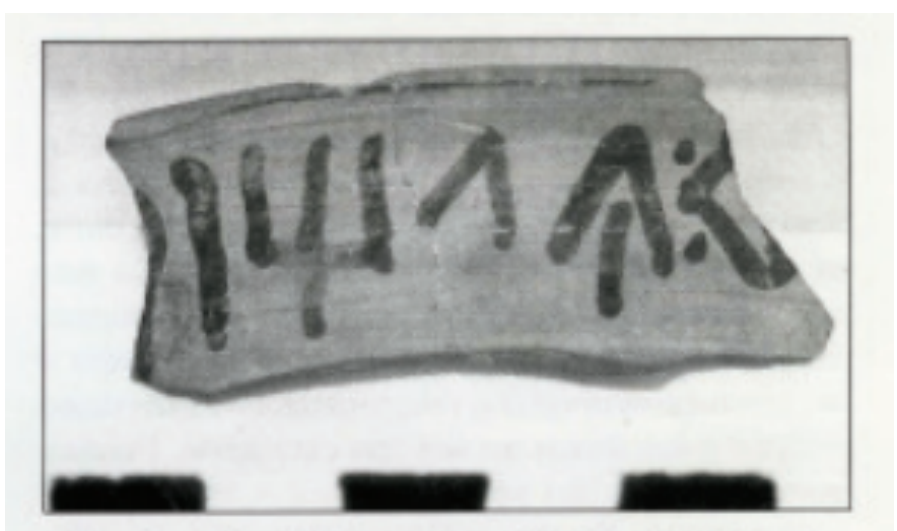

Figura 8. Gerardo Clausell Cantavella, ceràmica ibera amb una inscripció pintada, 2000

Al jaciment que hi ha a Borriana anomenat Torre d'Onda també va aparéixer una inscripció pintada sobre ceràmica, encara que és molt més breu. Molt possiblement es tracta dels signes se (Oliver, 1978: 272).

A la necròpoli del jaciment d'Almassora es van localitzar diverses urnes funeràries, nosaltres ens centrarem en la incineració 20. Es tracta d'una urna ibèrica feta a torn que s'havia col·locat dins d'un clot a la roca amb la intenció de dipositar-hi aquesta ceràmica. La peça és bastant gran, amb 35 centímetres d'alçada i un diàmetre màxim de 44 centímetres, tot i que la part superior està trencada segurament a causa dels treballs agrícoles. A l'interior, a banda de la cremació, hi havia dos peces més: el contenidor d'una segona incineració i un bol sencer. Totes tres tenen decoració pintada amb motius geomètrics i en les dues primeres hi ha decoració polícroma $\mathrm{i}$ vegetal (Clausell, 1999: 117-118).

A l'exterior de l'urna, superficialment, hi havia mostres dentals en bon estat de conservació. A l'interior hi havia les cendres dels difunts junt amb restes de la pira i també d'ovicaprí (Clausell, 1999: 118-119). Un dels dos grups de materials recollits en aquest soterrament correspon a una persona adulta jove, possiblement una dona, i l'altre grup, a un xiquet d'uns cinc anys (Clausell, 1999b: 504).

A la necròpoli de la Solivella també es van trobar urnes decorades amb pintura, fetes a torn, com és el cas de l'extreta en la sepultura número 25. És bitroncocònica, amb orelletes, i la decoració consisteix en figures geomètriques realitzades amb pintura rogenca. A l'interior hi havia l'aixovar, format en gran part per objectes xicotets de bronze. A més, té una tapadora feta amb pedra (Fletcher, 1965: 15, 25, 36).

Les urnes localitzades al Puig de la Nau eren ibèriques fetes a torn amb orelletes de diversos tipus. L'exemple que mostrem les té ambdues foradades i el cos és esfèric (imatge 38) (Oliver i Gusi, 1995b: 244). 
En les intervencions realitzades a la necròpoli del Torrelló també es van trobar altres materials. Quant al bronze, un exemple n'és la fíbula anular hispànica quasi perfectament conservada.

Al jaciment anomenat Puig de la Nau, hi van aparéixer també objectes fets amb bronze, com ara cadenes, segurament per a collars, sivelles per a la roba, anells o puntes de fletxa (Oliver i Gusi, 1995: 178-182).

Cal remarcar, però, l'aparició d'un escarabat etrusc a la necròpoli del Torrelló del Boverot (figura 9). El material utilitzat és pedra semipreciosa i se situa entre els segles V-IV a C. Les dimensions no són molt grans: fa 1,20 centímetres d'ample, 0,90 centímetres d'alçada i 1,50 centímetres de llarg del sòcol. Té una perforació perquè es puga encastar $\mathrm{i}$ al dors i sobre el sòcol es poden distingir les diferents parts de l'escarabat. En l'altra cara hi ha la representació d'una persona en moviment amb un casc, un escut i una llança. Davall de l'escut hi ha una planta i una sanefa cordejada que emmarca tota la figura (Clausell, 2003: 243).
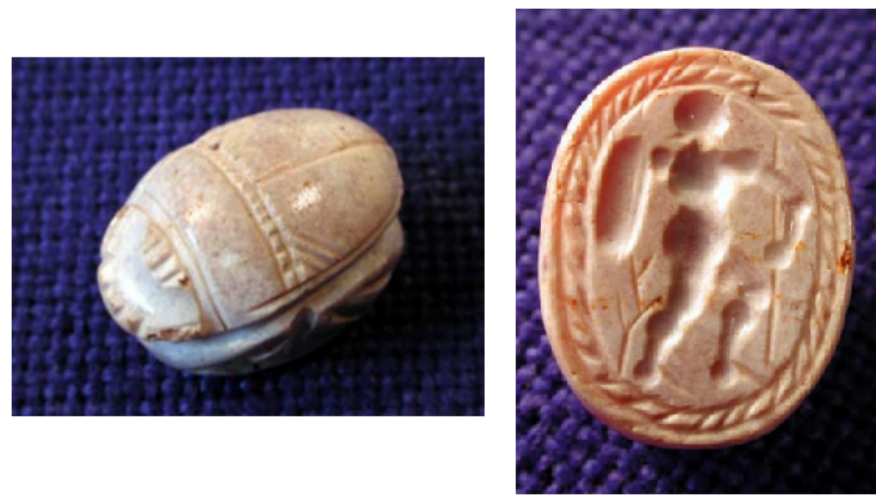

Figura 9. Gerardo Clausell Cantavella, cara externa (esquerra) i cara interna (dreta) de l'escarabat de la necròpoli del Torrelló del Boverot, 2003

I no és l'únic que s'ha trobat a la província. A la tomba 6 de la necròpolis de la Solivella també hi va aparéixer un a la tomba 6 . Fa 0,8 centímetres d'ample, 0,6 d'alçada i 1,2 de longitud. Es va fer amb pasta blanca vidriada a l'Egipte, té un forat longitudinal i està pitjor conservat, ja que està partit en dos trossos. Les figures representades no s'hi identifiquen tan clarament com les del Torrelló, l'escarabat apareix amb menys detalls i les figures de l'altra cara són teories. Possiblement hi ha un lleó tombat amb un element allargat entre les potes i per damunt té el disc solar (Padro: 72-73, 76).

\section{Discussió i conclusions}

A mesura que ha anat avançant l'elaboració del treball, s'han anat desenvolupant els objectius que s'hi havien establert, però també hi ha coses que han canviat. Es volia unir en un mateix 
document la informació més important en relació amb el Torrelló del

Boverot $\mathrm{i}$ tenir en compte altres jaciments amb característiques paregudes per tal de comparar materials i establir clarament les similituds cronològiques i culturals. Això no només s'ha acomplert, sinó que ha superat la idea original. El jaciment està format per l'anomenat Torrelló del Boverot, però també hi ha dues àrees que estan ubicades al terme municipal d'Onda: el Sitjar de Baix i la necròpoli. En un primer moment aquesta també es va tenir en compte, però no sabíem que existia l'altra zona i el fet d'haver trobat totes les parts que conformen el Torrelló suposa un èxit en la feina de recerca de tot tipus d'informació sobre aquest. Així doncs, aquest descobriment va permetre que també s'investigara sobre el Sitjar i que el treball fora més complet i fidel a la realitat.

Un altre objectiu ha sigut trobar altres jaciments que tingueren similituds cronològiques i culturals al Torrelló. El d'Almassora té diverses fases d'ocupació, però el treball se centra en l'Antiguitat. Així, hem tractat l'edat del bronze i l'edat del ferro, les etapes més rellevants en aquest cas. Quant al primer, per la seua ubicació estratègica, les estructures i els materials trobats -ceràmica amb les característiques típiques de la cultura de l'època i objectes de bronze- podem saber que va ser ocupat en aquest moment i a més es distingeixen fases d'abandonament intermèdies. Quant al segon, hi ha materials corresponents a la cultura ibèrica, majoritàriament ceràmica -per exemple les urnes amb orelletes- però també en destaca la influència dels contactes comercials mediterranis als pobles indígenes. A mesura que es va anar elaborant el treball es va comprendre fins a quin punt la influència fenícia inspira la cultura ibera, ja que la gent del llevant tenia la seua pròpia cultura i seguia el seu propi curs evolutiu, però amb aquests contactes s'accelera el canvi i s'acaben adoptant certs detalls d'estil ceràmic -que coneixen gràcies als fenicis- per a les produccions de la seua cultura.

Per a la recerca d'altres jaciments es va centrar la recerca en aquells que foren més pròxims i que tingueren més pareguts per a poder relacionar els materials del Torrelló amb els seus. És a dir, que es va escollir una mostra dels materials més representatius de cada època del jaciment d'Almassora i a partir d'aquests es va buscar als llocs que s'han exposat altres peces paregudes. D'aquesta manera es relaciona el Boverot amb el seu entorn i li dona més valor perquè es demostra que també és un jaciment que aporta informació valuosa i que cal tenir-lo en compte en els estudis generals de tota la zona castellonenca. Al començament del treball es va voler centrar la investigació a la zona del Millars, però finalment es va ampliar per a incloure altres jaciments molt importants de la província i perquè el d'Almassora no va rebre només la influència del riu, sinó que va estar en contacte amb les poblacions d'una zona més ampla i li van arribar 
les mateixes influències culturals que als altres poblats que hem comentat, independentment de la ubicació.

La bibliografia que s'ha necessitat per aconseguir l'objectiu que acabem de comentar ha sigut més accessible que en el cas anterior, però també és menys nombrosa quant a antecedents històrics. Algunes de les fonts han sigut manuals, dels quals hi ha molt pocs que es dediquen exclusivament a qualsevol de les dues èpoques i les cultures corresponents. Alguns manuals són de prehistòria i tenen apartats sobre aquests temes, i d'altres són d'una de les dues èpoques a què ens hem referit $i$ inclouen el llevant o es dediquen al bronze valencià $\mathrm{i}$ tenen algun apartat dedicat a un jaciment de Castelló. El que ha resultat més dificultós de trobar ha sigut bibliografia en altres idiomes. Aquest és un tema molt concret d'una regió molt concreta que ni tan sols abasta tota una comunitat, per tant només hi ha manuals generals per parlar d'un període en línies generals. Si els jaciments, per ara, no han sigut totalment estudiats pels autors locals, en part a causa de l'estancament dels projectes d'excavació, i recentment ha anat publicant-se bibliografia només sobre alguns de tots els casos, hi ha encara més dificultat per trobar autors d'altres països que dediquen els seus estudis i les seues publicacions a poblacions concretes de Castelló.

Quant a la bibliografia concreta sobre els jaciments, aquesta ha sigut més abundant i la major part l'hem trobada en forma d'article. L'únic problema ha sigut que alguns jaciments s'han tractat molt poc o en cap publicació científica i no s'hi han pogut incloure; així mateix, $n^{\prime}$ hi ha d'altres que fa temps que no s'excaven i, per tant, la bibliografia és massa antiga per a utilitzar-la, o també ens ha passat que només hi ha un dels articles que és mitjanament recent. En alguns casos només en trobàrem dels anys cinquanta, seixanta 0 setanta i en altres feien referència a publicacions dels anys trenta, per la qual cosa no es podia consultar la font original a l'hora de formular teories quant a la cronologia i la cultura perquè, amb el temps, han anat canviant. En definitiva, s'ha aconseguit aquest objectiu malgrat que s'ha pogut aportar molt poca bibliografia estrangera, s'hi ha inclós diversos jaciments arqueològics $i$, a més, s'ha tingut en compte la influència fenícia, reflectida en alguns materials.

M'agradaria esmentar la dificultat afegida que hi ha en relació amb la Torre d'Onda i el Torrelló d'Onda. La similitud terminològica pot induir alguns investigadors a confondre'ls o donar per fet que són el mateix. La Torre d’Onda està a Borriana, prop de la platja, i el Torrelló d'Onda s'ubica a Onda, vora el riu Millars. Faig aquest aclariment perquè en nombroses obres en citen un -més sovint la Torre d'Onda- de manera tan breu que no s'explica a quin municipi pertany, o també diuen que $\mathrm{n}^{\prime}$ hi ha un altre relativament proper amb 
una denominació pareguda i s'ha de parar atenció per no confondre els termes ni els jaciments ni creure que només n'hi ha un.

L'objectiu de comparar el Torrelló del Boverot amb altres jaciments conclou amb l'apartat dedicat a la cultura material. Originalment, es va pensar a comparar els jaciments sencers amb el d'Almassora, però l'extensió i el treball d'investigació haurien sigut excessius per a un Treball Final de Grau. Per aquesta raó es va apostar per la comparació dels materials, el bagatge cultural autèntic que reflecteix l'essència de cada època. Finalment, s'han comparat peces concretes del Torrelló amb altres que compartien més característiques mitjançant mostres de cada tipus de ceràmica i d'altres materials.

\section{Bibliografia}

Aguilella Arzo, Gustau. 2017. "Tossal del Mortòrum. Un assentament de l'edat del bronze i del Ferro Antic a la Ribera de Cabanes (Castelló)». Monografies de Prehistòria i Arqueologia Castellonenques 12.

Albelda, Vanessa i Sonia Machause. 2015. "L'Abric de les Cinc (Almenara): ¿Cuál fue su uso durante los siglos VII-IV a.C.?». Saguntum. Papeles del laboratorio de arqueología de Valencia Extra 17.

Alfonso Llorens, Joaquín i Helena Ruiz Conde. 2005. «Excavación arqueológica en el yacimiento del Sitjar Baix (Onda). Campaña 2005. Avance de los resultados». Quaderns de Prehistòria $i$ arqueologia de Castelló 24.

Arasa i Gil, Ferran i Norberto Mesado Oliver. 1997. "La ceràmica d'importació del jaciment ibèric de la Torre d'Onda (Borriana, La Plana Baixa)». Archivo de Prehistoria Levantina, XXII.

Barandiarán, Ignacio. 1998. Prehistoria de la Península Ibérica. Barcelona. Ariel.

Berrocal Ruiz, Paloma, et al. 2005. «Intervención arqueológica en el yacimiento arqueológico del Sitjar Baix, Onda (la Plana Baixa)». Quaderns de Prehistòria i arqueologia de Castelló 24.

Clausell Cantavella, Gerardo. 1987. «Excavaciones de salvamento en el Torrelló del Boverot d'Almassora». Quaderns de Prehistòria i arqueologia de Castelló 13.

-. 1997. «El comercio marítimo fenicio en la desembocadura del río Mijares (Castellón)", conferència presentada en les III Jornades d'Arqueologia Subaquàtica. València: Facultat de Geografia i Història de la Universitat de València, els dies 13, 14 i 15 de novembre de 1997. 
-. 1997b. «Materiales del Bronce Medio en el Torrelló del Boverot d'Almassora (Castellón)». La Murà.

—. 1998. «El comienzo de la iberización: el Torrelló del Boverot (Almazora, Castellón)». Quaderns de Prehistòria i arqueologia de Castelló 19.

—. 1999. "La incineración 20 de la necrópolis del Torrelló del Boverot (Almazora, Castellón)». Quaderns de Prehistòria i arqueologia de Castelló, 20.

-. 1999b. «Paralelos, cronología y estudio antropológico de la incineración 20 del Torrelló». Actes del XXV Congrés Nacional d'Arqueologia 501-507.

-. 2000. "La incidencia fenicia en el asentamiento del Torrelló del Boverot d'Almassora (Castellón)». Actas del IV Congreso Internacional de Estudios Fenicios y Púnicos IV.

—. 2003. «Un escarabeo etrusco en la n.ecrópolis del Torrelló del Boverot (Almassora, Castelló)». Quaderns de Prehistòria $i$ arqueologia de Castelló 23.

-. 2004. «El Torrelló del Boverot: del Bronce Medio al comienzo del Hierro». La Edad del Bronce en tierras valencianas y zonas limítrofes, coordinat per Mauro S. Hernández Pérez i Laura Hernández Alcaraz. Ayuntamiento de Villena i Instituto Alicantino de Cultura Juan Gil-Albert.

Clausell Cantavella, Gerardo, Isabel Izquierdo Moya, Ferran Arasa i Gil i Jordi Juan-Tresserras. 2000b. "La fase del Ibérico Final en el asentamiento del Torrelló del Boverot (Almazora, Castellón): dos piezas singulares». Archivo Español de Arqueología 73.

Fernández Castro, María Cruz. 1995. Iberian in Prehistory. Oxford: Blackwell.

Fletcher Valls, Domingo. 1965. "La necrópolis de la Solivella (Alcalá de Xivert)», Servicio de Investigación Prehistórica.

Gusi Jener, Francesc. 1974. «Excavación del recinto fortificado del Torrelló de Onda (Castellón)». Quaderns de Prehistòria i arqueologia de Castelló 1.

Junyent, Emili, et al. 1982-1983. "El Abric de les Cinc (Almenara, Castellón). 2a campaña de excavación 1977». Quaderns de Prehistòria i arqueologia de Castelló 9.

Mata Parreño, Consuelo. 2001. «Límites y fronteras en Edetania». Archivo de prehistoria Levantina XXIV.

Mesado Oliver, Norbert. 1974. Vinarragell (Burriana-Castellón). Valencia: Museu de Prehistòria de València. 
Oliver Foix, Arturo. 1978. «Epigrafía ibérica de la provincia de Castellón». Quaderns de Prehistòria i arqueologia de Castelló 5.

-. 2010. La Prehistòria en el Bajo Mijares. Castelló de la Plana: Societat Castellonenca de Cultura.

-. 2014. "La Necrópolis Ibérica de la Solivella: Nuevas visiones, nuevas propuestas». Cuadernos de Prehistoria y Arqueología 40.

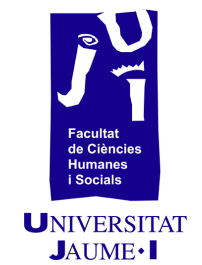

Oliver Foix, Arturo i Francesc Gusi Jener. 1995. «Análisis general de los materiales». El Puig de la Nau: un hàbitat fortificado en el ámbito mediterráneo peninsular 4.

-. 1995b. "La necrópolis». El Puig de la Nau: un hàbitat fortificado en el ámbito mediterráneo peninsular 4.

Padró i Parcerisa, Josep. 1974. "A propósito del escarabeo de la Solivella (Alcalá de Xivert, Castellón), y otras piezas egipcias de la zona del Bajo Ebro». Quaderns de Prehistòria i arqueologia de Castelló 1 



\section{Llengua i Literatura}

Lengua y Literatura Language and Literature 


per recuperar la dignitat

Noves propostes de cooperació a São Tomé i Príncep

Maria Montroy Ferré

maria.montroy@uji.es 
Aquesta investigació es planteja com una proposta per a analitzar la realitat sociolingüística i cultural de l'illa de São Tomé, a São Tomé i Príncep (Àfrica), i les possibilitats de cooperació en la Iluita contra la marginació que pateixen les persones majors i la desaparició de trets identitaris de l'illa, com ara el crioll forro i algunes de les tradicions (danses, celebracions, etc.). Partim de la premissa que la subtitulació al portugués de contingut audiovisual en forro pot ser una eina beneficiosa en la sensibilització de la població en l'àmbit de la cooperació. En aquest cas, parlem sobre l'abandonament que viuen els ancians i les ancianes a São Tomé i sobre el risc d'extinció del crioll forro amb tot el que això comporta: pèrdua d'aspectes culturals, desigualtats dels seus parlants respecte als monolingües de portugués, etc. Per tal de portar a terme l'objectiu de la investigació, es va realitzar una primera entrevista a tres ancians que parlen en crioll forro sobre qüestions socials $i$ culturals de São Tomé. Aquest vídeo es va subtitular al portugués i es va mostrar a un grup de persones de diferents edats i professions perquè servira de base per reflexionar sobre el que diuen els ancians i quina n'és la percepció de la població en general. A partir de les reflexions dels entrevistats, entenem que seria possible una cooperació alternativa, per exemple, la cooperació lingüística, que tinguera per objectiu revitalitzar el forro i, per tant, contribuiria indirectament a la sensibilització de la població cap a les persones ancianes i la cultura i les tradicions pròpies.

Paraules clau: São Tomé, forro, cooperació, ancians, subtitulació, Àfrica, recuperació lingüística, criolls, sociolingüística, etnografia.

\section{Introducció}

São Tomé i Príncep és un país format per diverses illes, situat al golf de Guinea, molt a prop de la línia de l'equador. Té una superfície de 1001 km i una població de 194000 habitants. El Terceiro Relatório Nacional dos Objectivos do Milénio de l'any 2014 indica una taxa de pobresa del $71,3 \%$ per a les dones i del 63,4\% per als homes i, pel que fa al desenvolupament humà, São Tomé i Príncep ocupa el lloc 127 en una llista de 175 països (Gonçalves de Género, 2012).

Quant a les llengües del país, podem dir que se n'hi parlen quatre a més del portugués: el lunguyé (exclusiu de l'illa de Príncep), el crioll capverdià, l'angolar i el crioll forro, aquest últim autòcton de l'illa de São Tomé (Hagemeijer, 2009). 
Es creu que, abans de la colonització per part dels portuguesos en el segle XVI, São Tomé estava deshabitada (Almada Negreiros, 1895; Seibert, 2009; Ribeiro de Souza, 2015). Per tant, les primeres llengües que s'hi van establir van ser, d'una banda, el portugués dels colonitzadors i, de l'altra, les llengües dels esclaus que es van portar des d'altres colònies per treballar, primer a les plantacions de sucre $\mathrm{i}$ més endavant, en els segles XIX i XX, a les de café i cacau (Hagemeijer, 2009; Gonçalves i Hagemeijer, 2015; Lorenzino 1996). De la barreja de totes aquestes llengües va sorgir un primer crioll que es va nativitzar ràpidament entre els descendents de la primera generació d'esclaus.

Com que cada poblador portugués tenia dret a una esclava per decreto régio, els fills d'aquestes unions que mesclaven el portugués patern i el primer crioll de les mares van ser els primers parlants de forro. També se'ls va començar a identificar amb eixe nom, ja que van ser declarats lliures mitjançant la carta de alforria a partir de 1515. Aquest crioll es va difondre ràpidament a les plantacions i es va convertir en la llengua que aprenien els esclaus que arribaven progressivament a l'illa (Hagemeijer, 2009).

Des que São Tomé i Príncep va aconseguir la independència de Portugal l'any 1975, el portugués es va consolidar com a llengua exclusiva (Gonçalves i Hagemeijer, 2015), ja que, tot i que la constitució del país no contempla la seua realitat lingüística (Gouveia, 1993), en la pràctica el portugués s'utilitza com a única llengua oficial. Així, l'estigmatització dels criolls no s'ha superat i ha suposat fins i tot una disminució d'alguns elements d'identitat de tipus cultural o religiós i que l'estatus del crioll nacional, el forro, siga de llengua en risc de desaparició (Ribeiro de Souza, 2015).

Actualment, les persones que mantenen el crioll forro com a llengua habitual són les persones que viuen a les zones més agrícoles i, sobretot, les persones de més edat (Mata, 2004; Ribeiro de Souza, 2015).

Per tal de caracteritzar el sector de la població parlant de forro, doncs, hem de tindre en compte dos factors: d'una banda, la distribució de la població a l'illa de São Tomé i, de l'altra, la situació i la percepció social de les persones ancianes a l'illa.

Pel que fa a la distribució poblacional, hi trobem dos àmbits diferenciats:

D'una banda hi ha la ciutat, que és on està la major part del comerç, el port, l'aeroport, l'hospital, la universitat, etc. Per aquest motiu, moltes persones procedents de zones més allunyades, van migrar a la ciutat amb l'objectiu de tindre més facilitats en l'àmbit laboral.

D'altra banda, tenim xicotetes comunitats per la costa i a l'interior de l'illa, i les antigues plantacions (roças). Algunes d'aquestes últimes s'han restaurat i s'han convertit en restaurants, 
hotels, etc., però moltes altres van quedar abandonades i s'hi han instal.lat famílies a viure, i les han convertit en una espècie de poble menut. Normalment, aquestes persones es dediquen al treball més agrícola i sovint es desplacen cada dia fins a la ciutat per a vendre fruites, verdures, peix, etc.

Quant a la situació social de les ancianes i els ancians, és important assenyalar que moltes de les actituds i característiques pròpies de l'edat avançada (insomni, tremolors, etc.) es relacionen a São Tomé amb la bruixeria i l'ocultisme. Aquest fet desemboca en la marginació i fins i tot el maltractament dels ancians, que es veuen completament desemparats (Valverde, 2000; Espírito Santo, 2009; Neves, 2009).

Potser un dels motius d'aquestes supersticions sobre els majors és que, en l'àmbit del curanderisme, sempre s'aprén d'una persona més vella. Es conten històries impressionants sobre els antics, hi havia alguns coneixements que només els més vells tenien. Uns diuen que no volgueren transmetre aquests coneixements. Altres, que va ser culpa dels joves, que amb la seua indolència i falta de respecte no van voler aprendre. Hi ha qui pensa que el desinterés dels joves va fer que els vells només transmeteren els seus sabers a uns pocs privilegiats (Valverde, 2000). A més, segons Valverde 2000), els curandeiros consideren un orgull ser poliglotes, perquè el fet de parlar moltes llengües els permet accedir a més esperits i obtindre més informació espiritual.

Moltes de les tradicions culturals de São Tomé s'han vist en detriment en els últims anys, la major part Iligades als ancians. Hi ha certs esdeveniments que ja no es duen a terme tan sovint com abans. Parlem de danses, festivitats, expressions culturals, etc. Molts joves ja no les coneixen, ni tampoc moltes de les músiques tradicionals, ni alguns plats de la gastronomia més ancestral (Amado, 2014).

Totes aquestes característiques de São Tomé motiven aquesta contribució per descobrir quina és la percepció de la població respecte a la situació de les persones ancianes, del forro i de les tradicions de l'illa. A partir de les respostes dels participants, podem plantejar noves propostes de cooperació per evitar que el crioll forro es perda i, amb això, pal-liar la situació dels ancians i la desaparició de trets culturals del país.

\section{Objectius}

L'objectiu d'aquesta investigació és analitzar les possibilitats de cooperació en el context de São Tomé per lluitar contra la marginació que pateixen els ancians i la desaparició de trets identitaris de l'illa, com ara el forro i les seues tradicions. Per tal d'assolir aquest 
objectiu, hem d'arribar a alguns objectius secundaris, tant teòrics com metodològics:

1. Descriure el context del forro, la situació dels ancians i les ancianes a São Tomé i les possibilitats de cooperació en aquest àmbit.

2. Esbrinar la percepció dels ancians sobre la situació que viuen al país i també sobre el detriment del crioll forro i de les seues tradicions per mitjà d'una entrevista enregistrada en aquesta llengua $i$ subtitulada al portugués.

3. Esbrinar la resposta que té un grup de persones de diferents edats, sexe i professions en veure el vídeo de l'entrevista als ancians i analitzar quina és la seua percepció de la situació del forro, de les persones ancianes i de les tradicions del país, per avaluar l'acollida que podria tindre una iniciativa de recuperació de la llengua.

\section{Metodologia}

Per tal d'analitzar la percepció de la població envers la llengua i cultura pròpies, però també envers la situació dels ancians del país, es va dur a terme un estudi qualitatiu i etnogràfic durant dos mesos. En una primera fase, es va enregistrar un vídeo on apareixien tres persones ancianes d'un centre de dia parlant en forro sobre com eren les tradicions del país i la vida dels ancians antigament. El vídeo es va subtitular al portugués per facilitar-ne la comprensió a les persones que el veurien després. En la segona fase de la investigació, es van entrevistar setze persones de professions diferents i d'entre 18 i 55 anys d'edat a partir del primer vídeo enregistrat.

\subsection{Participants}

Ancians: es va visitar un centre de dia de la ciutat de São Tomé. D'entre els vells que parlaven forro, dos dones i un home d'edats compreses entre els 70 i els 80 anys es van oferir a participar-hi. Els tres vivien a la ciutat, tot $\mathrm{i}$ que en barris diferents, $\mathrm{i}$ visitaven el centre per assegurar-se el desdejuni i el dinar diaris, ja que, tot i que els tres tenien fills, no podien atendre'ls gaire per motius que no van explicar.

Entrevistats: per a les entrevistes posteriors es va contactar amb setze persones de diferents edats (huit majors de 30 anys, huit menors de la mateixa edat) i professions: un cuiner, dos pediatres tradicionals, dues treballadores domèstiques, la directora de la Santa Casa da Misericordia, una empresària, una perruquera, un horticultor, una comptable, un guia turístic/barber/fotògraf, un 
estudiant, dos paletes, un atleta, una administrativa de l'hospital, un estudiant/paleta.

A causa de les dificultats per trobar persones que puguen dedicar el temps a realitzar aquestes entrevistes de manera altruista i sense generar desconfiances, es va començar a buscar per persones pròximes en l'àmbit personal i, gràcies a recomanacions d'aquestes primeres, es va poder accedir a la resta dels entrevistats, respectant sempre un equilibri entre l'edat i sexe dels participants i intentant que hi haguera varietat en les professions a les quals es dedicaven.

\subsection{Instruments}

\section{Entrevista als ancians:}

Tot i que es va deixar un cert marge de llibertat perquè pogueren parlar d'allò que volgueren, hi havia algunes preguntes de partida que els tres tenien en comú:

1. Com era la teua vida de menut/da?

2. Quines llengües parlaves més quan eres menut/da? I ara?

3. Saps alguna cançó en forro?

4. Les danses d'ara es fan igual que abans?

5. Pots parlar-nos d'alguna tradició del país? Continua celebrant-se igual ara i en el passat?

6. Com era la vida de les ancianes i els ancians en el passat? I ara?

\section{Vídeo subtitulat:}

El vídeo amb els comentaris i reflexions dels tres ancians tenia una duració d'uns quinze minuts $i$ es va gravar en forro per a, posteriorment, subtitular-lo al portugués.

No es va seguir una pauta establerta de subtitulació per tal d'adaptar-nos a la realitat del país: comptàvem que la majoria d'entrevistats només tindria estudis bàsics, per la qual cosa no estarien habituats a llegir. A São Tomé és molt difícil aconseguir llibres de lectura per a la major part de la població i, per tant, el nivell de lectura no seria el mateix que el d'una persona d'Europa. Així mateix, ens van comentar que no veien subtítols a la televisió habitualment i això ens va fer replantejar-nos la normativa habitual que presenta la subtitulació.

Per assegurar-nos que tots els entrevistats serien capaços de llegir els subtítols, es va agafar una persona que ens serviria de base, ja que entenia perfectament forro, no tenia estudis i tampoc no tenia costum de llegir. Amb l'ajut d'aquesta persona es va resumir la informació del vídeo al màxim per poder deixar els subtítols tot el temps possible en pantalla. Segons la dificultat del que s'estava dient, els subtítols es mantenien més o menys temps. Aquesta 
flexibilitat a l'hora de subtitular va permetre que totes les persones pogueren seguir el contingut del vídeo sense problemes, tot i que no es tracte d'una subtitulació convencional.

\section{Entrevista a la població:}

En primer lloc, als 16 entrevistats se'ls va fer una primera bateria de preguntes generals, que variava si l'entrevistat era nascut i criat a de preguntes gene
São Tomé o no:

Nascuts i criats a São Tomé:

1. Quina és la teua llengua materna?

2. En quina llengua parles als teus fills (si en tens)?

3. Quina llengua utilitzes més?

4. Amb quines llengües estàs en contacte en el teu dia a dia?

5. Quina creus que és la millor llengua per a l'ensenyament, anar al metge, fer gestions, etc.?

6. En quines ocasions es parla forro?

7. Com penses que és la vida de les persones ancianes a São Tomé?

8. Saps què són ladainha, txiloli, bócadú, danço congo, ússua, etc.? Has vist personalment aquestes coses? I els teus fills?

\section{Estrangers}

1. Quants anys fa que vius a São Tomé?

2. Vas necessitar aprendre alguna llengua per a viure ací?

3. Et relaciones més amb altres estrangers, amb locals o igual amb uns i altres?

4. T'has plantejat alguna vegada aprendre una altra llengua del país?

5. Saps quantes llengües s'hi parlen?

6. Quina has sentit parlar més (a banda del portugués)?

7. Alguna vegada has sentit parlar forro? En quin context?

8. Com creus que és la vida de les persones ancianes ací?

9. Saps el que són ladainha, txiloli, bócadú, danço congo, ússua, etc.? Has vist personalment aquestes coses?

Després, se'ls va mostrar a cadascun el vídeo dels ancians del centre de dia i, a continuació, havien de respondre una sèrie de preguntes:

Nascuts i criats a São Tomé:

1. Què opines del contingut del vídeo?

2. Coneixies alguna de les cançons que es canten al vídeo? Creus que els teus fills o les persones més joves les coneixerien? 
3. T'agradaria que el forro s'estudiara a les escoles o la universitat?

4. Per què creus que no es parla forro amb els fills?

5. Creus que hi ha una relació entre l'abandonament dels ancians i la pèrdua de llengua i tradicions del país?

6. Consideres important evitar-ho o és millor deixar que el progrés seguisca el seu curs?

7. Com creus que es podria fer?

\section{Estrangers}

1. Et sorprén el que conten els ancians?

2. Diuen que cada vegada es parla menys forro, que la llengua s'està perdent. Què n'opines?

3. Si es facilitara l'aprenentatge d'aquesta llengua, l'aprendries?

4. Coneixies alguna de les cançons que es canten?

5. Què et sembla que es perden algunes tradicions com el bócadú, les danses, etc.?

6. Creus que hi ha alguna relació entre l'abandonament dels ancians i la pèrdua de llengua i tradicions del país?

7. Com penses que podria evitar-se?

8. Consideres important evitar-ho o creus que és millor deixar que el progrés seguisca el seu curs?

\section{Resultats}

Abans de detindre'ns en les respostes que vam recollir, caldria explicar breument alguns esdeveniments culturals que es van esmenar en aquestes entrevistes:

El txiloli és una obra de teatre popular, d'aproximadament sis hores de duració, en les quals la música i la dansa són de gran importància. Hi ha una bibliografia àmplia i diferents estudis.

El danço congo i ússua són danses tradicionals que tenen els seus orígens en l'arribada d'esclaus a São Tomé des de diferents parts d'Àfrica.

El bócadú seria un equivalent al nostre dimecres de cendra. En aquest dia, era tradicional que les famílies es reuniren a dinar juntes $\mathrm{i}$ era el membre de més edat qui donava el menjar en la boca a la resta de la família.

Finalment, la ladainha és la forma antiga de celebrar els soterraments i passar el dol. Durant huit dies es menjava i bevia amb els familiars de la persona que havia mort i passaven aquests dies junts.

Una vegada aclarits els conceptes anteriors, podem passar a observar les respostes $i$ els comentaris més ressenyables dels tres ancians: 
1. Tenen la percepció que el forro és una llengua per a parlar en família i amb persones adultes, però amb els xiquets ha de parlar-se portugués. Un d'ells reconeix que només parlava forro fins que va tindre fills. Aleshores, va començar a utilitzar el portugués per parlar amb ells.

2. Un d'ells pensa que el forro hauria d'estudiar-se a l'escola, igual que el portugués.

3. Aprecien diferències en les danses tradicionals: ja no es toquen instruments tradicionals molt sovint $i$, segons la seua opinió, ara es balla per a guanyar concursos i competicions. Una de les entrevistades fa referència a les discoteques com una motivació per ballar distinta a la que ells tenien de joves. Pensen que alguns tipus de danses estan desapareixent.

4. Quant a altres tradicions, ens parlen del bócadú, del txiloli i la ladainha. El primer tenia els més vells com a protagonistes i era d'una gran importància familiar i en el veïnat; es preparava amb il/lusió. Del txiloli diuen que abans molts joves el veien i hi volien participar. Ara quasi ningú hi vol participar i els joves no tenen cap interés a veure'l. Consideren que la ladainha també desapareixerà prompte.

5. Pel que fa a com era la vida dels ancians en el passat, ens conten que abans les mares donaven menjar als xiquets perquè el portaren a les persones ancianes, les quals, com a agraïment, convidaven a menjar el xiquet i, de vegades, li donaven menjar per a la seua família; que eren molt bons i que se'ls parlava amb la mà al pit com a senyal de respecte. Ara, diuen, només se'ls acusa de fetillers i fins i tot els agredeixen.

A més, dues de les participants van voler cantar un fragment de dues cançons tradicionals, que pensen que ja no s'escolten tant com abans.

Quant a les respostes obtingudes de les setze persones que van veure aquest vídeo, podem extraure algunes conclusions generals:

1. Es considera que el crioll forro no és una llengua adequada per als xiquets. Es pensa que se'ls ha de parlar portugués $i$, quan siguen grans, tindran la responsabilitat d'aprendre forro pel seu compte, escoltant aquells qui ja la parlen i animant-se a utilitzar-la a poc a poc.

2. Tres dels entrevistats creuen que el forro s'aprendria millor a l'escola que a casa.

3. A tots els agradaria que la llengua s'estudiara a l'escola o universitat. Una d'elles assenyala que, si la llengua s'estudiara, ells serien "més aptes» en l'àmbit educatiu i en la seua vida en general. 
4. Dues persones asseguren que quan s'utilitzaven més les llengües criolles, les persones estaven més unides. Una explica el següent: "Com que abans el forro estava prohibit, parlar la llengua era el poder que tenia la gent, i això els unia. Però ara, aquesta llengua allunya els joves, que el que volen és aprendre anglés. Com que els joves s'han allunyat dels vells, és natural que també s'hagen allunyat de la llengua».

5. En general, es considera que el forro és la llengua dels proverbis, de les cançons, de fer bromes respecte a aquells que no coneixen la llengua, i dels ancians.

6. Tres dels entrevistats creuen que darrere de la discriminació dels ancians s'amaga també una discriminació econòmica: aquells a qui s'abandona, maltracta i margina sempre són ancians pobres.

7. Una entrevistada també assenyala que hi ha una diferència entre sexes a l'hora de discriminar ancians: mentre que hi ha més homes considerats curandeiros, són més les dones acusades de feiticera (fetillera).

8. Molts dels entrevistes pensen que la clau per no perdre ni la llengua ni les tradicions pròpies està a acostar-se als majors.

9. S'observa una diferència destacable entre els majors i menors de 20-25 anys respecte al coneixement que tenen de les tradicions i cançons populars. El txiloli és la tradició més coneguda entre ells, tot i que els més joves reconeixen no haver-lo vist mai personalment 0 , com a mínim, no sencer. El danço congo i ússua sí que eren danses reconegudes, però tots coincidien que ja no s'hi veuen tant com abans. Quant a la ladainha, cinc dels entrevistats desconeixien completament la seua existència, dos d'ells eren estrangeres i la resta menors de 26 anys. També tots coneixen el bócadú, però coincideixen que hi ha molt poques persones que ho celebren.

A banda, cal remarcar algunes de les aportacions de les persones que no han nascut a São Tomé. Una d'elles, empresària de professió, va respondre que coneixia "regular» la cultura del país, tot i que feia 25 anys que vivia a São Tomé i tindre'n la nacionalitat. Fora de gravació va comentar: "he dit que no conec bé la cultura del país perquè no he arribat a aprendre forro".

Un altre entrevistat de 22 anys nascut a Gabon comentava que ell es va esforçar per aprendre forro i que l'utilitza quan parla amb gent major o visita comunitats on sap que s'hi parla.

La tercera entrevistada estrangera, natural de Cap Verd, diu que és molt difícil aprendre una llengua que els seus parlants han 
abandonat. Pensa que si es facilitara l'aprenentatge del forro, ella l'aprendria i que aquells que se senten orgullosos de la seua llengua haurien de parlar-la. Assenyala també la diferència d'actituds entre les persones d'origen capverdià i aquelles d'origen forro respecte a la llengua i les tradicions: "Nosaltres sí que ens preocupem de mantindre la nostra llengua i les nostres tradicions».

Alguns dels participants han comentat que, durant els últims anys, hi ha joves que estan tornant a interessar-se per la llengua i les tradicions del país i intenten organitzar o participar d'activitats en aquest sentit.

Després de reflexionar sobre les seues respostes, es va preguntar als entrevistats si pensaven que hi havia algun tipus de relació entre la desaparició del forro i de moltes de les seues tradicions i l'abandonament dels ancians. Els setze van respondre que sí.

\section{Conclusions}

Segons les respostes obtingudes d'aquests setze entrevistats, i responent a l'objectiu d'esbrinar quina és la percepció de tots els participants sobre la situació, concloem que sí que hi ha un cert grau de sensibilitat per una part de la població sobre el que els passa als ancians i les ancianes; que s'adonen de la debilitació de les seues tradicions en els últims anys i que volen que es conserve tant la seua llengua com la seua cultura. Per tant, d'acord amb les respostes recollides, tot indica que, en cas de promoure treballs per a revitalitzar la llengua i les tradicions del país, la resposta de la població seria positiva i podria servir com un primer pas per a sensibilitzar sobre la situació de les persones majors.

A més, cal incidir en alguns apunts interessants que van comentar alguns entrevistats, que assenyalen la importància de les relacions familiars en la situació que viuen les ancianes i els ancians a São Tomé. Pensen que, a causa del passat colonial del país, on els esclaus no tenien cap nucli familiar -ja que l'interés dels amos perquè els esclaus tingueren fills responia únicament a assegurar-se una nova generació d'esclaus per a les seues plantacions-, els pares d'origen forro no són tan afectius amb els fills i, per tant, tampoc no saben respondre d'una altra manera quan els progenitors envelleixen. Segons aquestes persones, a això se li suma el fet que moltes famílies decideixen anar-se' $n$ a la ciutat $o$, fins i tot, a un altre país per motius econòmics. Llavors, els ancians i les ancianes de la família són una boca més a alimentar, que no aporta res a la casa i, com que no han rebut l'amor que s'esperaria en la infantesa, tampoc no els costa desentendre-se'n. De fet, una de les entrevistades deia que, en general, a São Tomé no es parla de sentiments. Tot i que ho presenta com una característica cultural, troba que això perjudica les 
relacions familiars i, en concret, la que es té amb les ancianes i els ancians.

En relació amb això, Valverde 2000) comenta que en forro no existeixen paraules per als sentiments com ara tristesa o alegria, sinó que només tenen paraules com sola (plor, plorar) o floga (broma, jugar, divertir-se...). Podria tractar-se del matís cultural del que ens parlen els entrevistats reflectit en la llengua.

Tot i que entenem que l'Estat hauria d'assumir la major part de responsabilitat per posar-ne una solució, no hem d'oblidar que a São Tomé hi treballen més de 150 ONG (Cravo et al., 2010) que intenten, com indica la mateixa definició de cooperació, treballar amb diferents actors per tal d'aconseguir el desenvolupament de les persones en tots els aspectes. Donats els resultats d'aquesta investigació, podem analitzar les possibilitats de cooperació per a treballar en favor de les persones ancianes, la llengua i les tradicions del país: caldria pensar a incloure un tipus de cooperació alternativa als projectes de São Tomé. En concret, es tractaria d'una cooperació lingüística que treballe en la revitalització del crioll forro amb l'objectiu, no només de protegir la riquesa cultural que comporta una llengua, sinó també de dignificar-ne els parlants. De la mateixa manera, també hi cabria una cooperació de tipus cultural, que acostara les cançons, les danses, els instruments, les històries i la gastronomia tradicionals als més joves, per intentar generar un interés que sembla perdut $\mathrm{i}$ intentar conservar-les. Aquest tipus d'iniciatives seria una manera indirecta de treballar contra l'estigmatització i la marginació de les persones majors en col-laboració amb altres entitats o altres tipus de cooperació que tinguen el mateix objectiu.

A més, aquestes collaboracions no només serien útils en la revitalització del crioll forro i la cultura de São Tomé, la qual cosa ja seria una fita important per a la riquesa cultural $i$ identitària que comporta, sinó que també podria utilitzar-se com a eina per a apoderar les dones, que són clau en la transmissió lingüística i cultural, i com també per a generar consciència ambiental, ja que és en la llengua on les comunitats conserven els coneixements ecològics més ancestrals (Abarrategi, 2016).

\section{Bibliografia}

Abarrategi Garaigordobil, Txema. 2016. «Un acercamiento a la identidad de cooperación. La cooperación lingüística como clave para la soberanía cultural». Revista Pueblos 70 (28 de juliol). Accés: http://www.revistapueblos.org/blog/2016/07/28/unacercamiento-a-la-identidad-de-la-cooperacion-la-cooperacionlinguistica-como-clave-para-la-soberania-cultural/ 
Almada Negreiros, José. 1895. História Ethnographica da Ilha de S. Thomé. Lisboa: Antiga Casa Bertrand. José Bastos.

Bacelar Gouveia, Jorge. 1993. "Constituição da República Democrática de S. Tomé e Príncipe». En As constituições dos estados lusófonos, 283-319. Lisboa: Notícias.

de Ceita do Espírito Santo, Armindo. 2009. São Tomé e Príncipe: problemas e perspectivas para o seu desenvolvimento. Lisboa: Colibri.

Cravo, Carolina; Laure Londaitzbehere, Olívio Diogo i Sónia Sousa. 2010. Estudo Diagnóstico das ONG em São Tomé e Príncipe. São Tomé: ACEP-Associação para a Cooperação entre os Povos. FONG-STP Federação das ONG em São Tomé e Príncipe.

Gonçalves, Rita i Tjerk Hagemeijer 2015. "O português num contexto multilingue: O caso de São Tomé e Príncipe». Revista Científica da Universidade Eduardo Mondlane: Série Letras e Ciências Sociais 11: 87-107.

Gonçalves de Género, Esterline. 2012. Análise da Estratégia de Desenvolvimento da União Africana: uma abordagem geo-social aplicada a São Tomé e Príncipe. Tesi doctoral. Lisboa: Universidade Técnica de Lisboa. Instituto Superior de Ciências Políticas e Sociais.

Hagemeijer, Tjerk. 2009. "As Línguas de S. Tomé e Príncipe». Revista de Crioulos de Base Lexical Portuguesa e Espanhola 1 (1): 1-27.

Lorenzino, Augusto Gerardo. 1996. «Uma avaliação socio-linguística sobre São Tomé e Príncipe». Conferència presentada en Congresso Internacional sobre o Português: Actas, vol. I-III, coordinat per Inês Duarte i Isabel Leiria. Lisboa: Associação Portuguesa de Linguística e Edições Colbri.

Mata, Inocência. 2004. "Uma questão de direitos linguísticos». En $A$ suave pátria: reflexões político-culturáis sobre a sociedade SãoTomense, 53-54. Lisboa: Colibri.

Montroy Ferré, Maria. 2018. «La cooperación lingüística en el ámbito de la cooperación internacional: el caso de Santo Tomé y Príncipe». Revista Pueblos (27 de setembre). Accés http://www.revistapueblos.org/blog/2018/09/27/lacooperacion-linguistica-en-el-ambito-de-la-cooperacioninternacional-el-caso-de-santo-tome-y-principe/

Neto Amado, Lúcio. 2014. Manifestações culturáis são-tomenses. Lisboa: Chiado Editora.

Neves, Paulo. 2009. Estórias de Vida contadas por idosos do centro de dia da cruz vermelha e do lar dona Simôa godinho da Santa Casa de Misercórdia de São Tomé e Príncipe. São Tomé e 
Príncipe: Uneos (União Nacional dos Escritores e Artistas de São Tomé e Príncipe).

Ribeiro de Souza, Luciana. 2015. «São Tomé e Príncipe em dois momentos identitários. (Quinta seção: discurso, língua, pátria e ensino)». Cadernos de Pós-Graduação em Letras (Mackenzie) $15(1)$.

http://editorarevistas.mackenzie.br/index.php/cpgl/article/view

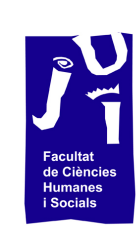

UNIVERSITAT JAUME・I 19428

Seibert, Gerhard. 2009. "Santo Tomé y Príncipe: transformaciones políticas y socioeconómicas del periodo poscolonial», traducció de María Puy. Nova Africa 24: 23-36.

Terceiro Relatório Nacional dos Objectivos do Milénio. 2014. São Tomé.

Valverde, Paulo. 2000. Máscara, Mato e Morte em São Tomé: textos para uma etnografia de São Tomé. Oeiras: Celta Editora. 
I. Abstract

\begin{abstract}
1. Abstract
A renewed interest has arisen on the importance of language attitudes in the acquisition of an additional language. Variables, such as age, gender, mother tongue, and the sociolinguistic context, have been taken into consideration when exploring language attitudes. However, the research on young learners' attitudes towards languages in English-based schools is scarce. The present paper focuses on young learners' language attitudes in a high English immersion context in the province of Castelló de la Plana, in the Valencian Community, where two official languages coexist, Spanish as the majority language, and Valencian as the minority language. However, English is the leading language of instruction in this international school context.

Many studies have been conducted within the framework of language attitudes across ages. However, as far as we know, no studies have accounted for young learners' attitudes in an English immersion context, such as international schools. Therefore, the present study aims at filling in this research gap by analysing children's language attitudes in an English immersion school context. The aims of this paper are twofold: in the first place, to analyse the language attitudes towards the three languages under investigation (Spanish, English and Valencian) of multilingual children attending an English immersion school. And, in the second place, to investigate the effect of age on language attitudes. The findings derived from the present study confirm the impact of age on young learners' language attitudes. However, the school context does not present a determining role in young learners' attitudes.
\end{abstract}

Keywords: language attitudes, high English immersion context, additional language, multilingual setting, young learners.

\title{
II. Introduction
}

When we talk about the acquisition of a second or an additional language, it is unarguably necessary to make reference to the factors that contribute to the process. One of the aspects that have always caught my attention is the way a language is acquired when we are not even able to stand on our own feet. In this process not only cognitive factors but also psychological factors that may be biased by outer and inner agents play a role. As noted by Portolés $(2014,2)$ "the affective side of languages is also paramount in order to better understand early language acquisition processes». One of these psychological factors that really caught my attention was the importance of attitudes towards languages and how the use of a first 
language may affect the way we acquire or learn an additional language. As part of human nature, somebody's acts, feelings and beliefs towards a language shape, both favourably and unfavourably, the way we see and, therefore, use and label a given language.

Many studies have been conducted within the framework of language attitudes across ages - children, adolescents, and adults. However, most of the research has analysed how native speakers of a language perceive, use and label their L1 or L2 within their speech community (Baker 1992). As far as we are aware, no studies have accounted for young learners' attitudes in an English immersion context, such as international schools. Therefore, the present study aims at filling in this research gap by analysing children's language attitudes in an English immersion school context. The aims of this paper are twofold: in the first place, to analyse the language attitudes towards the three languages under investigation (Spanish, English and Valencian) of multilingual children attending an English immersion school; and, in the second place, to investigate the effects of age on language attitudes.

We will first provide an explanation of language attitudes in a wider context, taking into account their definition as well as former studies conducted in relation to the topic of investigation. Then, the research questions will be posed together with the hypotheses. Afterwards, the setting, sample, instrument and procedure and data analysis will be carefully explained. Once the method has been presented, we will proceed with the analysis and discussion of the results obtained in the present study. The study will finish with a reflection on the results as a conclusion and the limitations that we found in the study as well as further research that can be done on the topic.

\section{Aims and research questions}

As far as we know, no studies have focused on multilingual children's language attitudes attending an English immersion setting. Hence, we aim at (1) analysing the language attitudes towards the three languages under investigation (Spanish, English and Valencian) of multilingual children attending an English-based school, (2) investigating the effect of the age on language attitudes. Taking into account the theoretical background and our aims, we have formulated the following research questions:

First research question (henceforth RQ1): What are the attitudes of very young learners towards different languages?

Second research question (henceforth RQ2): What is the effect of the age on their language attitudes? 
Considering the above research questions posed above, we have formulated the following hypotheses:

Hypothesis I: Students in English-based schools will display the most positive attitudes towards English, followed by Spanish and finally, Valencian.

Hypothesis II: Older students will show more negative attitudes towards the minority and the foreign language than younger learners.

\section{Methodology}

\subsection{Setting}

The study was carried out at an international school. The school is placed in the city of Vila-real, located in the province of Castelló, in the Valencian Community. This centre is a private educational centre which covers academic years at all levels; from nursery, infant, and primary, to secondary education and sixth form. The school offers students the opportunity to study the English National Curriculum. Additionally, the school offers updated facilities that benefit students learning and provide them with a high-quality educational experience.

In order to understand the setting as a high English immersion context, several important aspects as regards the school context have been deeply detailed hereunder according to Cenoz's (2009) continua of multilingual education, which are organised as follows:

Table 1. Analysis of the school according to Cenoz's (2009)

\begin{tabular}{|c|c|c|c|c|}
\hline $\begin{array}{l}\text { Subject (number of } \\
\text { languages courses) }\end{array}$ & $\begin{array}{l}\text { Language of } \\
\text { instruction (number } \\
\text { of languages to teach } \\
\text { contents) }\end{array}$ & $\begin{array}{c}\text { Teacher } \\
\text { (knowledge of } \\
\text { language) }\end{array}$ & $\begin{array}{l}\text { School context } \\
\text { (linguistic } \\
\text { landscape and } \\
\text { soundscape) }\end{array}$ & $\begin{array}{l}\text { Sociolinguistic context } \\
\text { (minority, majority } \\
\text { and foreign languages) }\end{array}$ \\
\hline $\begin{array}{ll}\text { - } & \text { English } \\
\text { - } & \text { Spanish } \\
\text { - } & \text { Valencian } \\
\text { - } & \text { French } \\
\text { - } & \text { German } \\
\text { - } & \text { Chinese }\end{array}$ & $\begin{array}{l}\text { - } \quad \text { English } \\
\text { - } \quad \text { Spanish }\end{array}$ & $\begin{array}{ll}\text { - } & \text { Spanish } \\
\text { - } & \text { Valencian } \\
\text { - } & \text { English } \\
\text { - } & \text { French } \\
\text { - } & \text { German } \\
\text { - } & \text { Chinese }\end{array}$ & $\begin{array}{ll}\text { - } & \text { Spanish } \\
\text { - } & \text { English } \\
\text { - } & \text { Valencian }\end{array}$ & $\begin{array}{ll}- & \text { Spanish } \\
-\quad & \text { English } \\
& \text { Valencian }\end{array}$ \\
\hline
\end{tabular}

Concerning the language of instruction used among the teachers, English is the main language used for communication in the school. As aforementioned, almost all content subjects are taught in 
English, except for those regarding language courses, which are taught in the target language. Table 3 shows the percentages of languages instruction in the school.

Table 2. Percentages of language instruction

\begin{tabular}{|l|c|c|c|c|c|c|}
\hline & English & Spanish & Valencian & French & Chinese & German \\
\hline Nursery & $90 \%$ & $10 \%$ & - & & - & - \\
\hline Primary & $60 \%$ & $30 \%$ & $10 \%$ & - & - & - \\
\hline Secondary & $60 \%$ & $10 \%$ & $10 \%$ & $(10 \%)$ & $(10 \%)$ & $(10 \%)$ \\
\hline Sixth Form & $50 \%$ & $30 \%$ & - & $(10 \%)$ & $(10 \%)$ & $(10 \%)$ \\
\hline
\end{tabular}

As shown in Table 2, most content subjects are taught in English, while Spanish is only used in primary and sixth form to teach content subjects such as social science or business. Additionally, Valencian is introduced in primary and taught through Key Stage 2 and 3 (i.e. primary and secondary). Also, a third language is introduced in secondary education. However, the rest of the subjects are taught in English such as Maths, Geography, History, Arts and Crafts or PE. Hence, all teachers in the school know English as well as personal staff. However, not all native speakers of English can speak Spanish, for instance, there is a number of teachers who do not understand Spanish language. In addition to teachers and staff, students are all multilingual. For example, there are Chinese, African, and English students who have to deal with more than three languages everyday, as well as those students who are Spanish or Valencian native speakers. In fact, those children who use Spanish in the classroom are always told to use only English both to speak with the teacher and with the classmates.

When referring to the language of instruction, there are three main languages - English, Spanish, and Valencian. On the one hand, if we focus on the classroom context, English is the only language used with the exception of the language used in language subjects, such as Valencian, Spanish, French, German, or Chinese, where the target language is the language used. As regards teachers' meetings, English is also the language of communication. However, there are some Spanish native speakers who use Spanish to express personal opinions or talk about important circumstances concerning students, as it is the case of the teacher of Valencian in primary education. On the other hand, the language used to address parents is usually Spanish since most of them do not speak nor understand English. Still, parents are able to access a website where they can be updated on their children's exams, homework, or field trips. In this way, parents are able to choose between English or Spanish as the school website offers both versions. However, if parents want to arrange a 
tutorial with an English native speaker and this particular professor is not able to carry out a conversation in Spanish, there is always another professor who interprets the tutorial. Despite the English language being the majority language in formal contexts, the school offers several extracurricular activities which are not necessarily taught in English such as robotics or ICT.

Finally, we observed the sociolinguistic context, which comprises the way languages are used in terms of majority and minority languages or heritage. As mentioned, Valencian is hardly used in the school aside from native children who talk in Valencian during break time or Valencian teachers who may refer to other Spanish teachers using the language in hand. Nonetheless, the language is not used if the interlocutor is not sure whether the other person involved in the conversation is a Valencian native speaker. Hence, Spanish would be the language chosen for communication in these situations. In primary education, as previously stated, students use the English language to communicate with each other and with the teacher. Nonetheless, students in secondary education hardly use English to talk with their classmates but only to address the professor. Additionally, children would mainly use Spanish with each other outside the classroom. As the vast majority of professors are English native speakers, English would be the language used for communication with each other. Therefore, the English language is the majority language at the school followed by Spanish, whereas Valencian is the minority language. Foreign languages (i.e. Chinese, German, French), however, are only present within the language courses as included in the school's curriculum.

On the whole, the British School in which the present study was conducted should be considered a multilingual school and, thus, it holds a high English immersion context.

\subsection{Participants}

All the participants in this study $(\mathrm{N}=70)$ were primary students from a single private school in an industrial town in the Valencian Community. In order to carry out the study, two classes from the first grade (mean age: 5.5 ) as well as two classes from the fifth grade of primary education (mean age: 8.5) have been examined for the study. Nonetheless, they all share a similar socio-economic status (high-class). As Table 3 presents, more than a half of the students come from fifth grade $(n=42 ; 60 \%)$ whereas the rest of the participants are currently enrolled in the first grade of primary education ( $n=28 ; 40 \%$ ). Moreover, $57 \%$ of the students in Year 1 are female and $43 \%$ are male, while the majority of participants from Year 5 are male students (55\%) and the rest are female (45\%). 
Table 3. Total number of participants

\begin{tabular}{|l|c|c|}
\hline & Year 1 & Year 2 \\
\hline Male & 12 & 23 \\
\hline Female & 16 & 19 \\
\hline $\mathrm{N}=$ Students & $28(40 \%)$ & $42 \%(60 \%)$ \\
\hline Total & & 70 \\
\hline
\end{tabular}

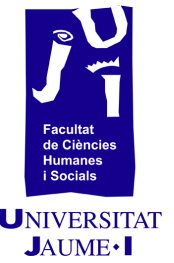

The respondents in this study have different languages as a mother tongue, although the majority of them speak Spanish or Valencian as their first language. In order to better illustrate these data, Figure 1 displays the different L1 of students.

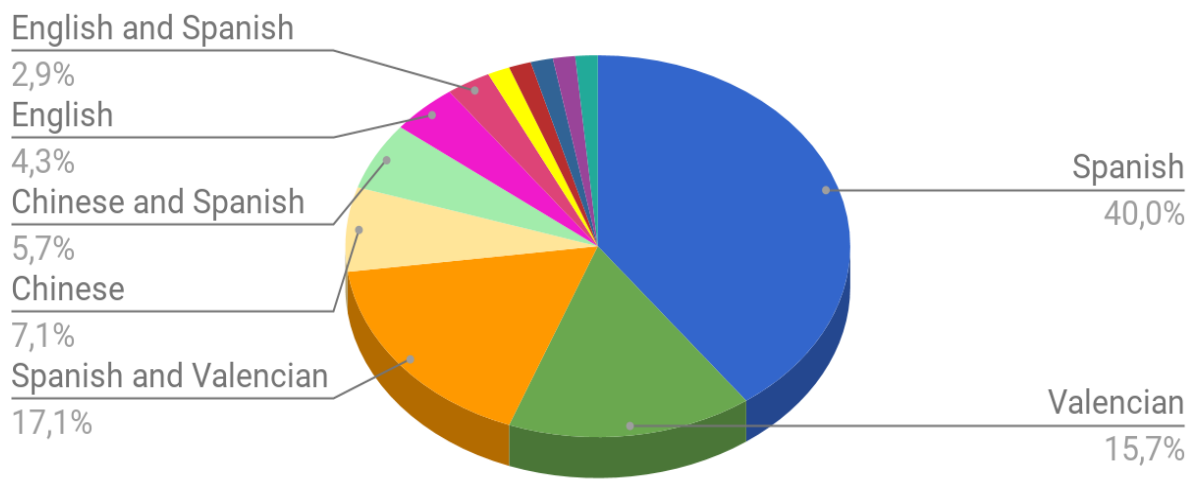

Figure 1. Students' L1

Figure 1 highlights a great difference between those students who noted to have Spanish as their mother tongue $(n=28 ; 39 \%)$ and those who stated to use Valencian as their first language $(n=11$; $16 \%)$. However, $17 \%(n=12)$ of the students considered both Valencian and Spanish as their mother tongues. When students were asked the reason why they considered to have both languages as a first language (L1) they all remarked that they use one language with one parent and the other language with the other parent. As regards the mother tongue of the other $45 \%$ of the students, almost $13 \%$ $(n=9)$ spoke Chinese as a first language, either together with Spanish or not.

\subsection{Instrument}

The research instrument used to gather the data was based on Portolés (2014) and adapted for our purposes. Therefore, in order to analyse children's language attitudes towards the three languages (Spanish, English and Valencian), we employed a matched-guise technique. First of all, participants were asked to write personal information such as their names, age and first language. Considering the age of students, we made use of red and green stickers to report 
young learners' language attitudes. As stated in Portolés (2014), these colours hold positive and negative connotations as they are directly linked with the colours of traffic lights. However, we omitted the use of the yellow colour, that was used by Portolés (2014) to portray neutral choices and, therefore, only positive and negative language attitudes will be analysed within our study. The questionnaire included three pictures of the two main characters of the story with a blue, a pink and a green background. These three colours respectively made reference to the language used to tell the scripts of the story; the blue picture made reference to Spanish, the pink picture to English, and the green picture to Valencian (see Appendix A).

The script was adapted from a current Disney film that became very popular among the younger population in the recent years Frozen. The script (see Appendix B) was adapted and translated into the minority language (Valencian), the majority language (Spanish) and the foreign language (English). The translation was carefully done in order to have the final result as similar as possible in the three languages.

According to the criteria of the matched-guise technique, only one person should be representing the different speeches to avoid external variables affecting the participants' responses. In this line, the script was read aloud by the main researcher of the present study, native speaker of Valencian and Spanish, and proficient in English. The tone, intonation, and emphasis were thoughtfully taking into consideration.

\subsection{Procedure and data analysis}

The data collection procedure took place in an English-based school set in the province of Castelló de la Plana, where this study was conducted. First of all, the deputy director of the school was informed about the purposes of the study. After that, I explained the aims of the thesis and the time that would be needed to conduct the activity to the different teachers. Once I received their consent and we agreed on a date for the implementation of the task, we started with the data collection.

To start with, we printed out two questionnaires in each sheet of paper and then we cut it out in a half. As a result, a hundred A3-sizequestionnaires were printed, from which seventy-one were used. Additionally, the three pictures used in the matched-guise questionnaire were printed in A4 format. As aforementioned, questionnaires were distributed to each of the students together with three green stickers (positive attitudes) and three red stickers (negative attitudes) that they had to put next to the three different pictures. These questionnaires were identical for Year 1 and Year 5 students. 
Before starting with the activity, students were provided with the explanation of the task and the instructions to follow. Moreover, they were explicitly told not to copy their classmates' answers, while they were supervised by their tutor and the main researcher during the whole activity to make sure that their answers were individual choices. Once the activity was explained and understood, students proceeded to write their names, ages and mother tongue. Then, the researcher showed the A4 picture that corresponded to the audio track that was about to be played. In this way, students could follow the activity and know where they had to put the sticker. Finally, a pen-drive with three different audio tracks was used to play the recordings in the class computer.

The implementation of the activity was conducted in the classrooms and it lasted a total of four days; the first two days we collected the data in both of the classes in Year 5 and the last two days in those of Year 1 . The length of the activity varied depending on the class, with a higher demand of time when dealing with the younger students. Hence, around thirty minutes were needed to finish the activity in Year 1 and fifteen minutes in Year 5 (see Table 4).

Table 4. Duration of the implementation of the study

\begin{tabular}{|c|c|}
\hline Academic year & Duration \\
\hline Year 5 A & 15 \\
\hline Year 5 B & 15 \\
\hline Year 1 A & 30 \\
\hline Year 1 B & 30 \\
\hline Total & $1{\mathrm{~h} 30^{\prime}}$ \\
\hline
\end{tabular}

The answers collected were codified in an Excel page in order to consider the different variables for analysis. In the process of codifying the data, we found out that one student used half of a green sticker and half of a red sticker to illustrate a «neutral» answer. Despite the fact that they were told not to use both, this student did not consent to use either a red or a green sticker to determine his attitudes towards Spanish. Therefore, this data had to be removed from the data analysed. This is the reason why we only considered seventy questionnaires instead of seventy-one that were distributed in the very beginning. This being solved and all the valid data codified, percentages were employed in order to perform the qualitative analysis, which is presented and discussion in the next section. 
Considering the research questions presented in the method section, we will start by analysing the results in order to answer RQ1 in the first place. To start with, global results will be analysed taking into account both academic years as a whole. In this vein, language attitudes in an English-based school will be determined. Then, in the RQ2, we will continue by considering each academic year individually in order to examine to what extent the variable of the age may affect the way children perceive different languages.

\subsection{Results and discussion related to hypothesis I}

With the main concern of analysing the language attitudes of school children in an English-based school context, we have posited Hypothesis I, which claims that pre-schoolers and primary students would display more favourable attitudes towards Spanish, followed by English and finally, Valencian. In line with this assumption, we examined the global percentage of positive language attitudes towards the three languages under investigation. As it can be observed in Figure 2, these findings suggest that the participants' overall attitudes towards languages are highly positive as $71.9 \%$ of the responses reported favourable attitudes.

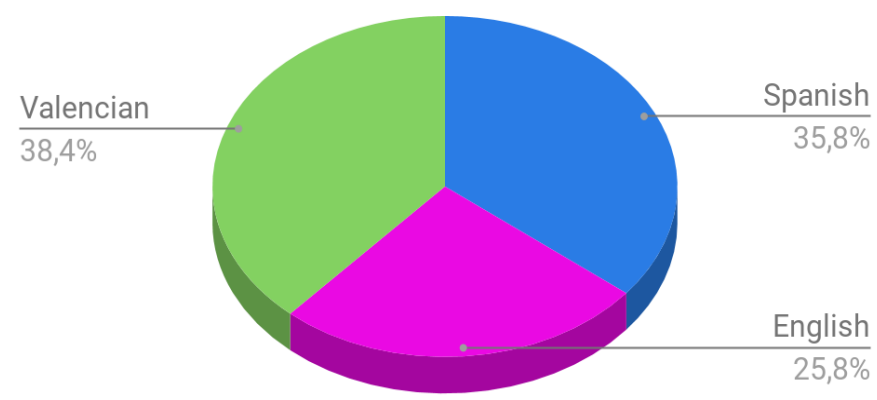

Figure 2. Students' positive language attitudes

The overall results of positive language attitudes do not seem to provide a significant difference between Spanish ( $n=54 ; 35.8 \%)$ and Valencian ( $n=58 ; 38.4 \%)$. In fact, the Figure shows a preference towards Valencian rather than towards Spanish. With reference to English, more unfavourable attitudes are portrayed in the findings as only $25.8 \%(n=39)$ of the students noted to have favourable attitudes. Contrary to our previous expectations, the minority language reported the most positive attitudes, followed by Spanish, and finally English. Our study contradicts Portolés' (2014, 144) findings in which participants appeared to have higher favourable 
attitudes towards Spanish than towards the other two languages under investigation, that is Valencian and English. However, our findings are in line with Nightingale's (2012) study on adolescents' language attitudes, who reported to have more positive language attitudes towards Valencian in the first place, followed by English and finally Spanish. Still, the foreign language (i.e. English) is placed in the last place in the present study, even though the context is an English-based school.

As aforementioned, teachers at the school were also impressed with the findings since students' everyday attitudes towards the minority language are far more negative that what we found in our results. In fact, one of the teachers claimed that even Valencian native speakers are reluctant to use Valencian in an educational context and they all resort to the use of Spanish or English to address teachers or other students. Moreover, we believe that other variables which have not been taken into consideration in the present study might have played a role in students' choices. The order in which the recordings were played may have also influenced the way children perceived each recording in the different languages. As Spanish was the first audio track to be played, this might have caused the better understanding of the story in Valencian, that is, the third audio track.

It is important to highlight that according to our findings, children's behaviour do not match the language attitudes' affective component. While their behaviour shows negative attitudes towards Valencian, the affective part shows the contrary. That is to say that respondents displayed very positive attitudes towards Valencian but then they do not use it, probably due to the low prestige that Valencian has in the present school. The same happened in O'Rourke and Ramallo's (2015) study about Galician speakers' language attitudes, who displayed commitment and very positive attitudes towards the language (i.e. cognitively and affectively) but very low language practices (i.e. the behavioural component). In line with these findings, it can be stated that the behavioural and the affective component of the tripartite attitudes' construct can be analysed individually.

Taking into account our findings, the hypothesis is not confirmed and, therefore, our previous expectations have been contradicted since our participants displayed more favourable attitudes towards Valencian, followed by Spanish and finally, English.

\subsection{Results and discussion related to hypothesis II}

The second research question seeks to determine whether the age variable may have an impact on young learners' language attitudes (Baker 1992, Nikolov 1999, Mihaljević-Djigunović and Krevelj 2009, Nightingale 2012, Portolés 2014) in an English-based 
school context. Our hypothesis II claims that younger learners would display more positive attitudes towards the three languages than those in older learners. In order to test the second hypothesis, Figure 3 illustrates the overall positive attitudes from Year 1 and Year 5 students.
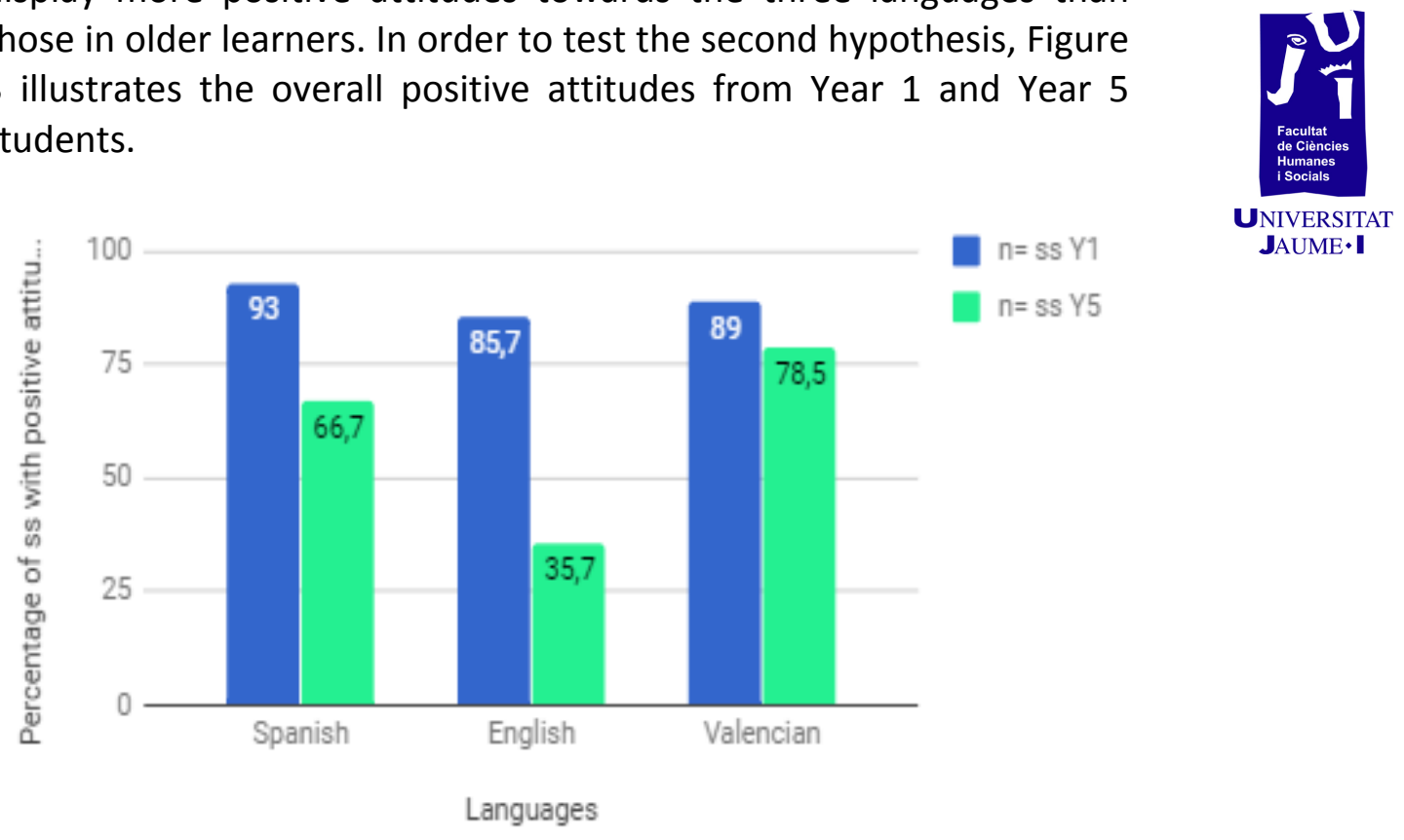

Figure 3. Students with positive attitudes in Year 1 and Year 5

As it can be observed in Figure 3, there seems to be a significant difference between the amount of positive language attitudes shown in Year 1 and Year 5. At first sight, first grade school children have the most positive attitudes towards the three languages. As opposed to the overall positive attitudes analysed in the previous subsection, this figure presents that younger learners show higher positive attitudes in Spanish (93\%) than in Valencian (89\%). However, English follows Valencian really close as positive attitudes refers. Yet, it is still presented in the last place $(85.7 \%)$.

Considering the results regarding school children in the fifth grade, the reported attitudes are quite lower than those presented in first grade. Year 5 students show a clear preference for Valencian (78.5\%), followed by Spanish (66.7\%) and finally English (35.7\%). It is important to highlight that the younger learners, those whose ages range from five to six years old, always speak in English with each other as well as with the teacher. However, as they grow older they start using Spanish to talk with their classmates and only resort to English when they address to the teacher, this can be clearly observed in secondary or sixth form. Self-awareness and self-esteem might be some of the key factors that influence language use within puberty. Therefore, students' attitudes towards the foreign language seem to be affected by the age variable since we can observe a great different between younger learners' (85.7\%) and older learners' (35.7\%) attitudes towards English. 
These results, thus, are in line with other studies' findings (Baker 1992, Nightingale 2012, Portolés 2014). Portolés (2014, 147) also found out that preschoolers show a preference for the minority and the foreign language when compared to primary students results. She also claimed that "young children lack awareness of the low status and vitality of the minority language" and, thus, this is reflected in the present results since younger learners' displayed high positive attitudes towards the minority and the foreign language compared to the older learners.

As stated by Portolés $(2014,150)$, «Baker (1992) reported that favourable attitudes towards the minority language decrease with age». However, this is not the case in the present study since fifth grade students showed more favourable attitudes towards the minority language (78.5\%) than to the majority language $(66.7 \%)$. In this vein, it cannot be stated that the majority language has got the higher positive attitudes as regards older learners. Thus, these findings contradict previous research (Hoffmann and Ytsma 2004, Lasagabaster and Huguet 2007, Rindler Schjerve and Vetter 2012, Portolés 2014) in which respondents reported to have more favourable attitudes towards the majority language (i.e. Spanish) as they grew older, than to the minority or the foreign language. These authors also claimed that Spanish is considered to have a higher prestige in shared bilingual communities and, therefore, it is the language used in formal situations or places considered to be of high social status. In line with these assumptions, if we consider our respondents' language practices, Spanish is the language mostly used among them in the school, followed by English. We probably agree on the fact that this might due to the high prestige that Spanish has got in the present school context.

Taking into account these results, the hypothesis II is not confirmed since older students' showed more negative attitudes towards the majority and the foreign language than younger learners. Therefore, as discussed in the previous section, the affective and behavioural components do not match either since older learners seem to have positive feelings towards Valencian (i.e. positive language attitudes) but they rather use Spanish or English to communicate at the school.

\section{Conclusions}

Language attitudes may play an important role in the process of learning an additional language and, therefore, they may affect the degree to which we are able to master it. Existing research (Safont 2007, Nightingale 2012, Portolés 2014) has examined learners' language attitudes. However, no studies have been conducted in English-based schools. 
Thus, the present study aimed at covering that research gap by examining young learners' language attitudes in an English immersion context. Variables, such as age have been taken into consideration. Our results, as regards the age variable, show that positive language attitudes drop over time and, thus, these findings are in line with previous studies (Baker 1992, Nikolov 1999, Cenoz 2009, Lefever 2009, Nightingale 2012, Portolés 2014). Taking these findings into consideration, it cannot be stated that the sociolinguistic context (i.e. the English immersion school context) necessarily affects the development of language attitudes across the years.

However, considering our results, the minority language gains favourable attitudes as regards older learners while majority and foreign languages' attitudes decrease over time. Considering the school context as a paramount element affecting language attitudes and leaving the age variable out of the equation, it can be noted that it may affect language attitudes in a certain way especially on a daily basis. For example, students do not use Valencian in class and are quite reluctant to receive instruction in this language as stated by several teachers in the school. Nonetheless, this is not reflected on our results since the minority language reported the most favourable attitudes, followed by Spanish and, finally, English.

Our findings suggest that, although the language attitudes' tripartite construct can be analysed by considering its three components to be interrelated, they can also be analysed individually since sometime what we feel or believe does not match with actual responses or behaviours.

In our view, these results have provided valuable insights that should be further investigated by applying statistical methods in order to get determining results.

\section{References}

Baker, Colin. 1992. Attitudes and language. Clevedon: Multilingual Matters.

Cenoz, Jasone. 2009. Towards Multilingual Education. Basque Educational Research from an International Perspective. Bristol, UK: Multilingual Matters.

Hoffmann, Charlotte, and Johannes Ytsma, eds. 2004. Trilingualism in Family, School and Community. Clevedon: Multilingual Matters.

Multilingualism in European bilingual contexts: Language use and attitudes.

Lefever, Samuel. 2009. "When I wanna be cool ...": English for young learners in Iceland." In Young Learner English Language Policy and Implementation: International Perspectives, edited by 
Janet Enever, Jayne Moon and Uma Raman, 103-112. Reading: Garnet Education.

Mihaljević-Djigunović, Jelena, and Stela Letica Krevelj. 2009. "Instructed Early SLA: Development of Attitudes.» Studia Romanica et Anglica Zagrabiensia : Revue publiée par les Sections romane, italienne et anglaise de la Faculté des Lettres de l'Université de Zagreb 54: 137-156.

Nightingale, Richard. 2012. Bridging the gap between the internal and external: The Effect of Sociocultural Factors in Adolescent Learners' Attitudes Towards English. Saarbrücken: Lambert Academic Publishing.

Nikolov, Marianne. 1999. "Classroom observation project.» In English Language Education in Hungary: A baseline study, edited by Marianne Nikolov, Hajnal Fekete and Éva Major, 221-246. Budapest: British Council.

O'Rourke, Bernadette, and Fernando Ramallo. 2015. «Neofalantes as an active minority: Understanding language practices and motivations for change amongst new speakers of Galician.» International Journal of the Sociology of Language 231: 147-165.

Portolés, Laura. 2014. «Analysing Prospective Teachers' Attitudes towards Three Languages in Two Different Sociolinguistic and Educational Setting." In Teaching and Learning in Multilingual Contexts: Sociolinguistics and Educational Perspectives, edited by Agnieszka Otwinowska and Gessica De Angelis, 50-67. Bristol, UK: Multilingual Matters.

Rindler Schjerve, Rosita, and Eva Vetter. 2012. European Multilingualism Current Perspectives and Challenges. Bristol: Multilingual Matters. Bristol: Multilingual Matters.

Safont, M. Pilar. 2007. «Language Use and Language Attitudes in the Valencian Community." In Multilingualism in European Bilingual Contexts: Language Use and Attitudes, edited by David Lasagabaster and Ángel Huguet, 90-113. Clevedon: Multilingual Matters. 



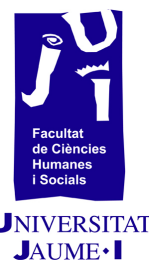

\section{Analysis of the correlation between self-perceived linguistic competence and language attitudes in the Valencian multilingual context}


I. Abstract

Language learning, language use and language attitudes are narrowly interrelated concepts, whose significance becomes even more relevant in diglossic settings involving a majority, a minority and a foreign language. This is precisely the fractured linguistic context of the Valencian Community, where the burgeoning role of English in the educational system adds to the asymmetrical bilingual setting in which the autochthonous language is minoritized in its own territory. Therefore, drawing on multilingualism and language attitudes as the theoretical framework, this study provides an interface between both concepts by focusing on the correlation between self-perceived competence and language attitudes in the Valencian multilingual setting by secondary education students.

As part of more extensive research examining the correlation between several variables and language attitudes, this paper builds on the previous findings on language attitudes in Catalan-speaking areas. It analyses the impact of self-assessed competence in both the majority and minority languages (Catalan and Spanish) on language attitudes towards the three languages amongst a sample of $624^{\text {th }}$ year ESO students from the Catalan-speaking area of the Valencian Community. From a quantitative approach, this cross-sectional study presents a statistical analysis of the gathered data, searching bivariate correlations to determine the correlation between both variables. The analysis of the results and the following conclusions highlight the correlation between language proficiency and language attitudes as well as the determinant role of linguistic command to promote balanced language attitudes and to achieve effective bilingualism.

Keywords: language attitudes, self-perceived competence, asymmetrical bilingualism, diglossic context, multilingualism.

\section{Introduction}

The international sociolinguistic landscape has changed dramatically over the last century partly fuelled by the increasing number of multilingual individuals and societies to the extent of multilingualism emerging as an all-pervading phenomenon (Gorter 2006, Auer and Wei 2007, Todeva and Cenoz 2009, Cenoz 2013). Thus, although the number of multilingual individuals and communities overcoming those being proficient in only one language is not a recent occurrence (Cenoz and Genesse 1998, Dewaele 2005, Safont 2007), it was in the middle of the 20th century that it began to catch the attention of researchers and to create a corpus that 
continues to increase (Herdina and Jessner 2002, Gorter 2006,

Pavlenko 2007, De Angelis and Dewaele 2011).

As pointed out by Alcón and Safont $(2013$, cited in Portolés 2015) the growing interest in this phenomenon can be mainly attributed to two different factors: the expanding role of English as a lingua franca and the revitalization of minority languages.

Notwithstanding this fact, despite unequally effective linguistic policies to bolster language revitalization, the evident linguistic imbalance between majority and minority languages and their different official and social status is alarming (Extra and Gorter 2001, Phillipson 2004, 2006, Romaine 2007). Spain, that holds about $50 \%$ of the 20 million speakers of minority languages in the European framework (Lasagabaser 2017), presents a wide context for the study of multilingualism, affective factors and language learning and uses, as it is shown by the numerous studies conducted in the bilingual autonomous communities (Lasagabaster 2000, 2003, 2005a, Pascual 2006, Huguet 2007b, Lasagabaster 2007, Safont 2007, Portolés 2011, 2015, Safont 2015). Nevertheless, this study will focus on the Valencian Community, a highly diglossic setting that had been neglected until a few years ago when authors started to turn their attention to this multilingual context (Safont 2007, Portolés 2011, Nightingale 2012, Portolés 2014, Safont 2015, Nightingale 2016).

As regards the role of English as a Lingua Franca (ELF), as stated by Cenoz and Jessner (2000), although its global pervasiveness is widely assumed, its interface in conjunction with or in opposition to minority languages is contentious (Phillipson and Skutnabb-Kangas 1996, House 2003, Phillipson 2004, Dewey 2007, Portolés 2015). In the case of the Valencian Community, it plays a paramount role in the educational system wherein English is the first and most widespread foreign language.

When dealing with languages, it is essential to adopt a holistic approach since they cannot be isolated from their speakers, nor from the socio-cultural context wherein they are used and wherefrom they stem. Actually, context is credited with a widespread impact on the speakers that will further leverage their attitudes towards other languages and their own. It is against this multilingual background, that the importance of language attitudes emerges. The role of affective factors is paramount in educational contexts in multilingual settings involving a majority, a minority language in process or revitalization and a foreign language (Lasagabaster 2003, 2005a). As it has been widely stated (Dörnyei 1990, Baker 1992, Garrett 2001, Dörnyei 2003, Huguet and González-Riaño 2004, Lasagabaster 2005b), attitudes held towards a language will influence both its learning process and its future use on the part of the learners. Language attitudes are also clearly influenced by the social perception of a language and its use in society (Riley 2007, Todeva 
and Cenoz 2009, Bou 2016), even though these attitudes can be learnt and modified by means of effective language and language-ineducation policies.

However, bilingual and multilingual policies and language attitudes are unfortunately not always in line with scientific and linguistic research. This is the case of the Valencian Community, where effective bilingualism is less widespread than it could be expected in a region in which two languages, Spanish and Catalan, hold co-official status.

\section{The Valencian multilingual context}

The Valencian Community is a highly diglossic context, wherein the majority language (Spanish) coexists with Catalan, the autochthonous minority and minoritized language, in an asymmetrical bilingual setting. In this diglossic frame, a foreign language, English, comes on the scene, thus creating a multilingual context with three languages in contact.

Although the Valencian Community is a bilingual region, in which both languages, Catalan and Spanish, share official status, effective bilingualism is not the norm. If both co-official languages status and social widespread are compared with the linguistic context in other autonomous communities sharing both the same official languages, such as Catalonia or the Balearic Islands, the minority position of Catalan in favour of the dominant language, Spanish, is evident. This linguistic imbalance in the Valencian Community, Catalan being the minority language in its own historical territory has raised increasing interest in recent years (Safont 2007, González-Martínez 2010, Calatayud 2011, Portolés 2014).

The attempts at developing effective bilingualism amongst students resulted in a wide array of linguistic programs, partly influenced by the two linguistic regions in the Community, the predominantly Spanish-speaking and the Catalan-speaking areas. This division, first established by the Statute of Autonomy (Generalitat Valenciana 1982) and adopted by the LUEV one year later (art 6.7), has strongly determined future language and language-in-education policies as well as it has perpetuated linguistic conflict impeding symmetrical bilingualism (Torró 2001, Pascual 2006, Bodoque 2011, Pascual 2011, Arnau and Vila 2013). Furthermore, the fact that the presence of Catalan is unequal through all rungs of the educational ladder does not help promote a cohesive concept of the language nor booster its social status which results in an unsteady disparity in the use of the vernacular language.

Thus, this diglossic context and fractured educational model leads to strong divisions between Spanish and Catalan native speakers, language educational models based on the majority or 
minority language and an unequal command of both the co-official languages according to the specific sociolinguistic features of the students. Consequently, language attitudes are strongly polarized between two linguistic groups, which in turn determine language learning and language use.

This study is set in the Catalan-speaking territories, where two different programmes co-exist in secondary education. The Spanishmedium model, "Programa d'incorporació progressiva» (PIP), wherein Spanish is the main language of instruction and the Catalanbased model, "Programa d'Ensenyament en Valencià» (Valencian Teaching Programme, hereafter PEV), which uses Catalan as the vehicular language.

\section{Objectives}

The current study aims to shed light on the research on language attitudes in the multilingual context of the Valencian community, a sociolinguistic setting generally neglected until the last decades. Following the path initiated by Safont's (2007) and Portolés' studies (2011), who started filling the gap on research in language attitudes in the Valencian Community from a multilingual approach, this study focuses on the impact of self-assessed linguistic competence in both the co-official languages, Spanish and Catalan, on language attitudes towards the three languages present in the curriculum: Catalan, Spanish and English. The variable 'linguistic competence' was selected due to its relevance in previous studies that place it amongst the most definitely agreed upon influential factors on language attitudes (Baker 1992, Janés 2006, Querol and Huguet 2010).

As pointed out by Lasagabaster and Sierra (2009) "the more positive the students' attitudes, the higher their L2 achievement». Thus, taking into account that there is a "bidirectional correlation» between language proficiency and language attitudes (Lasagabaster 2005a, Nightingale 2012, cited in Portolés 2015) we will evaluate students' self-evaluated competence both in the majority and the minority language and its impact on language attitudes. The research question and the consequent hypothesis which will guide the study are as follows:

RQ1: Does students' self-assessed competence in both the coofficial languages have an impact on their attitudes towards the three languages in contact?

$\mathrm{H} 1$ : There is a correlation between students' self-assessed competence in both the co-official languages and their attitudes towards the three languages in contact. 
This is a cross-sectional study which follows a quantitative method, based on the standards of validity, reliability, replicability, generalizability (Brown 1988) to gather the data and analyse the results obtained. According to Brown and Rodgers' (2002) classification, this study is a primary research that contains both a survey research (including the use of questionnaires and Likert scales) and a descriptive statistical research. The obtained data were analysed using the Statistical Package for the Social Sciences (SPSS).

\section{Participants}

The sample was constituted by $624^{\text {th }}$-year ESO students (15-16 years old) from "Les Alfàbegues» state high school in Bétera, a medium-sized village located in a predominantly Catalan-speaking area. This is a heterogeneous group and the pupils study any of the two $4^{\text {th }}$-year ESO branches: Academic and Applied Education, which will gear them to Batxillerat or Vocational Training studies respectively, in both the linguistic programmes offered in the school, the Valencian-based (PEP) and the Spanish-based (PIP) models. This duality regarding the linguistic models was one of the reasons to select this as the setting for our study since it was the only institution of secondary education in Bétera where the variable linguistic model could be observed.

Regarding the features of the sample, their distribution according to gender is quite balanced, $51.61 \%$ of the respondents being female and $48.39 \%$ male. Nevertheless, it is striking to notice that although there is maintenance in the percentage of students following the Catalan-based model both in primary and secondary school (57.38\%), the exclusive or nearly exclusive use of the minority language only represents $9.62 \%$ of social interactions with friends at school, a percentage that drops when talking to friends outside school (1.9\%). In fact, a majority of students reported using only or mostly Spanish at home, In social interactions with relativea and strangers, as well as with their peers and friends, inside $(67.31 \%)$ and outside school (76.92\%). These data are in line with the language students self-identify, which is mainly Spanish (61.29\%), followed by Catalan (19.35\%) and both (12.9\%).

As for the learning of the foreign language, the average age they started learning English was 5.74 years old. It is significant to observe that averagely, students began speaking Spanish earlier (2.85 years old) than they started learning Catalan (4.45). 


\section{Research instruments}

The research instruments used to collect and analyse the data involved four questionnaires filled in by the students which included a sociolinguistic survey, a self-assessment of their linguistic competence in both the co-official languages, and sets of questions to evaluate their language use and their attitudes towards the 3 languages in contact. These questionnaires had been successfully used in previous research in bilingual and multilingual contexts both in the Catalan-speaking regions and abroad (Huguet and Llurda 2001, Laoire 2007, Lasagabaster 2007, Safont 2007, Portolés 2011).

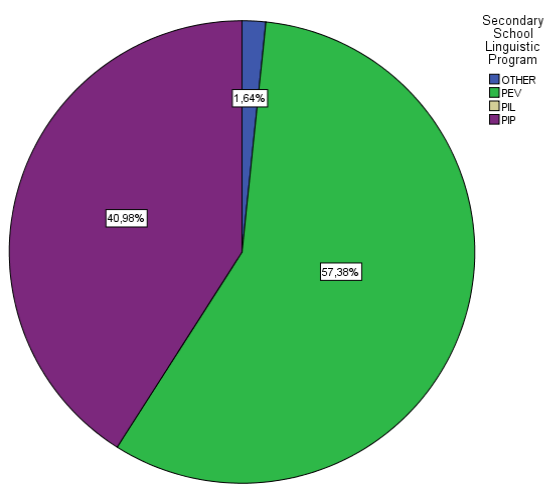

Figure 1. Gender and primary and secondary linguistic programs

The first instrument (sociolinguistic survey) consists of 24 questions which mainly gather data about students' linguistic background, linguistic educational program, language transmission, familiar geographical origin and parent's educational and socioprofessional status. The self-assessment questionnaire to gauge student's self-perceived competence both in Spanish and Catalan has been adapted from previous questionnaires that analysed language attitudes in the Valencian Community (Safont 2007, Portolés 2011). It consists of 8 questions, 4 per language to assess students' own perception of their language competence in every skill (listening, reading, speaking and writing). It uses a Likert scale from 1 to 10, in contrast with the 5-Likert rating questionnaire to measure their language attitudes towards the three languages in contact (questionnaire 3). To analyse the student's use of the two co-official languages, the third questionnaire consists of 19 multiple choice questions which were adapted and designed to gather data on several social interactions and situations wherein both languages are used. Finally, the last questionnaire gathers data on language attitudes towards Spanish, Catalan and English. To this aim, it includes the same 10 statements for each of the three languages, 30 in total, to be measured by means of a 5-Likert scale.

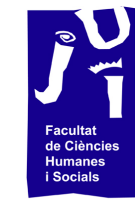

UNIVERSITAT JAUME $\mathbf{I}$ 
Before analysing the correlation between self-assessed linguistic competence and language attitudes, I analysed and compared student's self-perceived competence in both the co-official languages.

The first graph displays the self-perceived linguistic competence in the majority language, wherein both the mean and the median are significantly higher (9.13 and 9.50) as opposed to the linguistic competence in Catalan, showed in the second graph, where the mean and the median drop to 7.87 and 7.90 respectively.

It is also noticeable the difference between the ranges in the majority and the minority languages. Thus, the general range in selfperceived linguistic competence in Catalan is much wider (5.75) than the general range in the majority language, 4.75. This difference becomes even more evident when we eliminate the isolated data in both languages, in which case the range of self-assessed competence in Spanish drops to 2.25 whereas in the minority language the range reaches 4.50 .

According to the previous analysis, it can be deduced that students perceive to possess a higher command of the majority language in contrast to their self-perceived lower proficiency in the minority language. Furthermore, it is noticeable that participants' values of self-perceived competence in Catalan are more spread out and therefore there is a greater variability amongst students' responses.

This great variability in the data regarding self-assessed linguistic competence in Catalan led me to analyse the distribution of linguistic competence in the minority language in deeper detail to find out the segment of the sample where we can find this disparity.

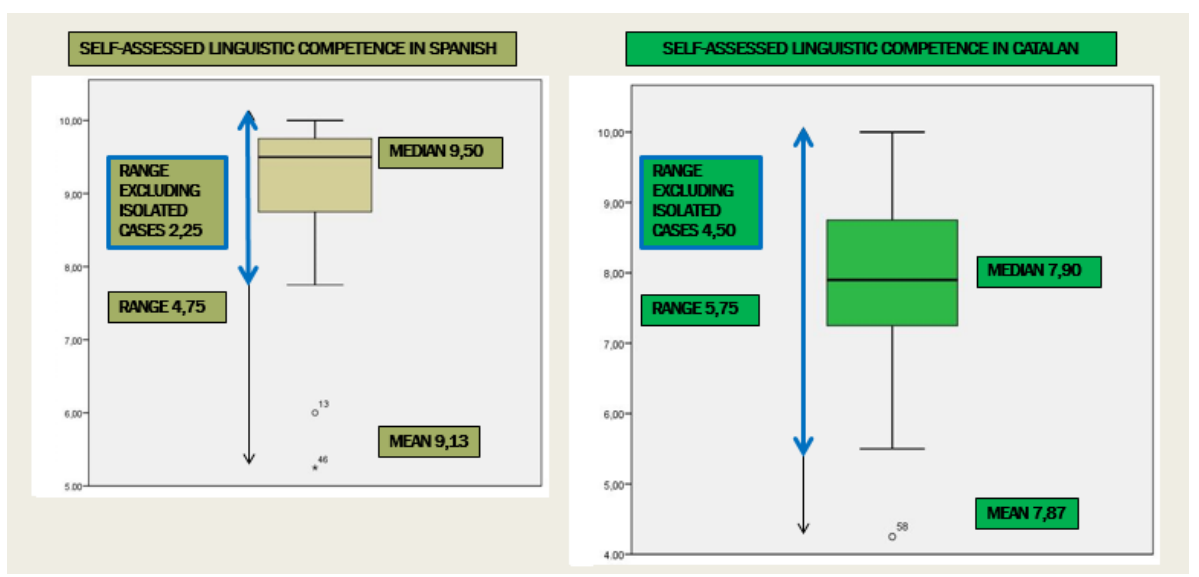

Figure 2. Self-assessed linguistic competences distribution 
The graph below shows the analysis of the distribution of students' language attitudes according to their mother tongue, Spanish, Catalan or self-perceived bilinguals in both languages.

It can be observed that, as could have been expected, the wider variability in self-perceived linguistic competence in Catalan can be found amongst students whose mother tongue is Spanish. In fact, except for the isolated data, the global range coincides with the range of Spanish-native speakers. On the contrary, the range is lower and self-perceived linguistic competence in Catalan values are higher amongst those students who have Catalan as their only or at least as one of their mother tongues.

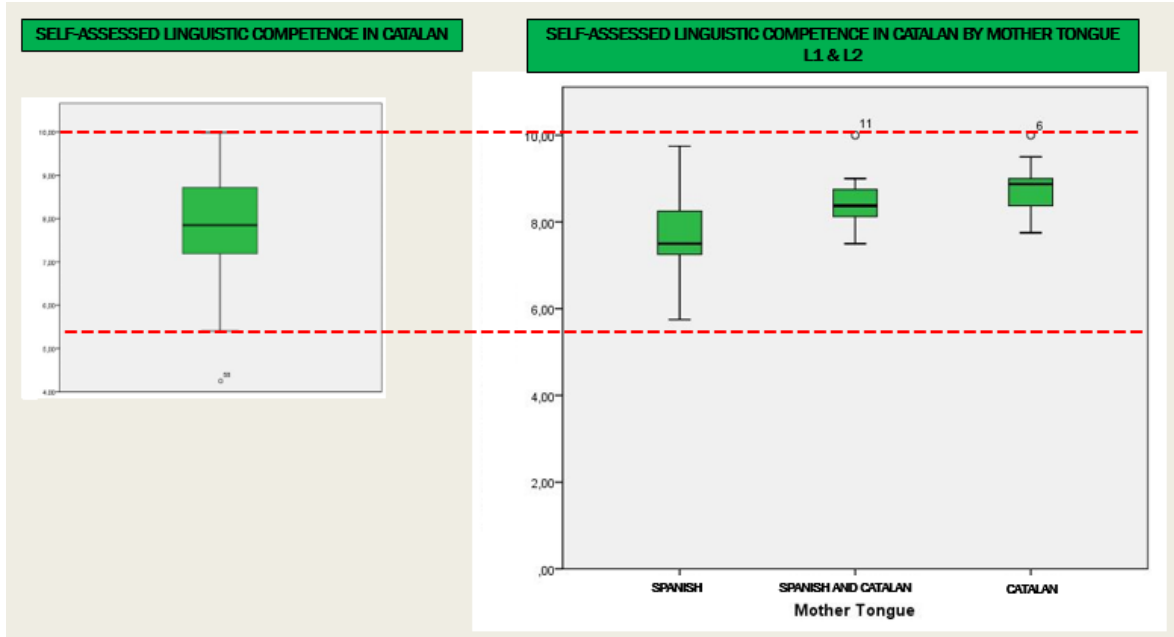

Figure 3. Distribution of self-assessed competence in Catalan by mother tongue

After having observed the students' self-assessed competence in both languages, I analysed the data statistically regarding language attitudes towards the three languages examined in this study, obtaining a mean of 4.06 (L1), 3.74 (L3) and 3 (L2) as shown in Table 1. The results show that students hold more polarized attitudes towards L2 (Catalan), their variance being 0.92; as opposed to 0.54 in Spanish (L1) and 0.60 in English (L3). This polarization in attitude is especially noticeable in the segment of respondents that hold much less favourable attitudes towards Catalan, as reflected in the fact that the $25^{\text {th }}$ percentile regarding $L 2$ is further from the mean than the corresponding percentiles regarding the other analysed languages. Furthermore, according to the rank analysis it can also be deduced that the respondents display a wider range of attitudes towards the minority language. 
Table 1. Attitudes towards languages

\begin{tabular}{|l|l|l|l|l|l|l|}
\hline & $\begin{array}{l}\text { Mea } \\
\mathrm{n}\end{array}$ & $\begin{array}{l}\text { Percentile } \\
25\end{array}$ & $\begin{array}{l}\text { Media } \\
\mathrm{n}\end{array}$ & $\begin{array}{l}\text { Percentile } \\
75\end{array}$ & $\begin{array}{l}\text { Rang } \\
\mathrm{e}\end{array}$ & $\begin{array}{l}\text { Varianc } \\
\mathrm{e}\end{array}$ \\
\hline $\begin{array}{l}\text { ATTITUDES } \\
\text { TOWARDS } \\
\text { CATALAN }\end{array}$ & 3.03 & 2.40 & 2.90 & 3.70 & 3.79 & .86 \\
\hline $\begin{array}{l}\text { ATTITUDES } \\
\text { TOWARDS } \\
\text { SPANISH }\end{array}$ & 4.05 & 3.50 & 4.10 & 4.60 & 3.00 & .53 \\
\hline $\begin{array}{l}\text { ATTITUDES } \\
\text { TOWARDS } \\
\text { ENGLISH }\end{array}$ & 3.75 & 3.30 & 3.70 & 4.20 & 3.20 & .52 \\
\hline
\end{tabular}

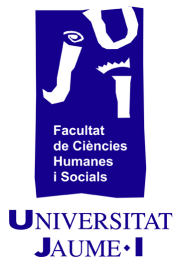

Thus, as depicted in Figure 4, more positive attitudes were displayed towards Spanish and less favourable ones towards Catalan. It is remarkable that attitudes towards the foreign language, although still scored lower than those towards the majority language, were still more favourable than attitudes towards Catalan. In fact Catalan is the language towards which the students hold the least positive attitudes.

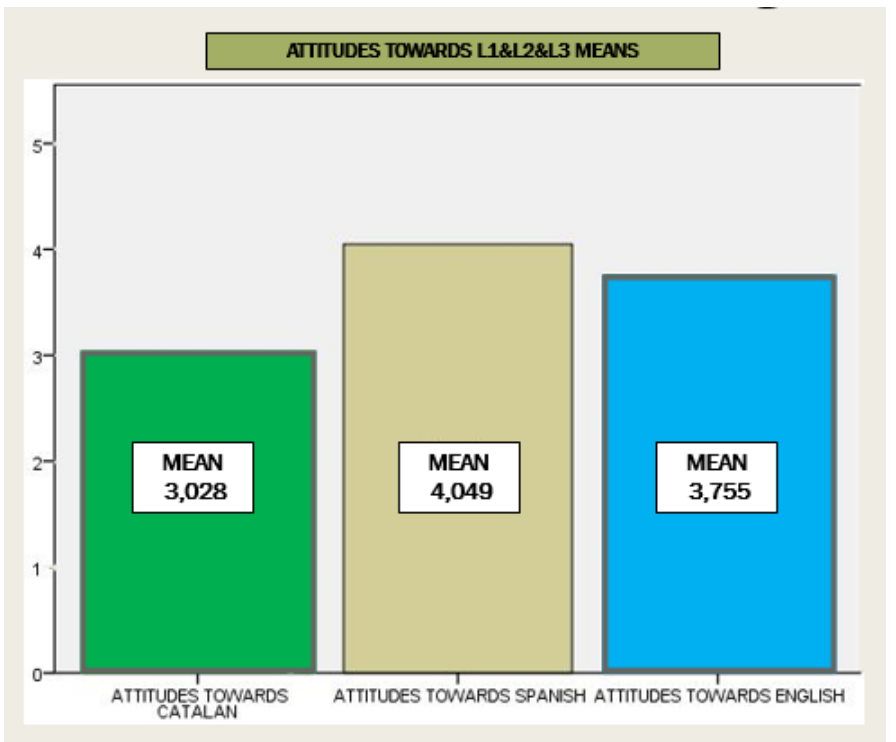

Figure 4. Attitudes towards L1, L2 and L3 means

Once I had studied students' self-perceived linguistic competence in both the co-official language, their distribution according to mother tongue and their attitudes means towards the three languages involved, I analysed the proposed research question that guided this study. To answer it I had to compare 6 different combinations which were the result of determining the influence of the self-perceived competences in L1 and L2 in each of the three languages object of study.

I consider that the verification of at least 4 of these combinations between the three languages in contact and the self- 
assessed competence in the two co-official languages will result in the confirmation of the hypothesis.

To test the 6 combinations I carried out a bivariate correlation to determine the linear relationship between the variables, comparing them in pairs, where the obtained Pearson correlation coefficients indicate the strength of the linear relationships between the pairs (Table 2. Correlations attitudes - self-assessed competences). The pairs whose correlations have significance in the level 0.01 are highlighted in dark colours, whereas those in light colours, display significance in the level 0.05. 5 out of the 6 correlations tested were verified; thus being 3 direct correlations (coloured in blue) and 1 indirect correlation (coloured in red) established. Only one of the analysed pairs was assessed as no correlated (no coloured). It is significant that self-assessed linguistic competence in one of the co-official languages is directly correlated to attitudes towards this language but indirectly correlated to attitudes towards the other co-official language. These results reflect the linguistic conflict between both the majority and the minority languages.

Table 2. Correlations attitudes - self-assessed competences

\begin{tabular}{|c|c|c|c|c|}
\hline & & $\begin{array}{l}\text { ATTITUDES } \\
\text { TOWARDS } \\
\text { CATALAN }\end{array}$ & $\begin{array}{c}\text { ATTITUDES } \\
\text { TOWARDS } \\
\text { SPANISH }\end{array}$ & $\begin{array}{c}\text { ATTITUDES } \\
\text { TOWARDS } \\
\text { ENGLISH }\end{array}$ \\
\hline \multirow{2}{*}{$\begin{array}{l}\text { Self-assessment of } \\
\text { linguistic } \\
\text { competence in } \\
\text { Catalan }\end{array}$} & $\begin{array}{c}\text { Pearson } \\
\text { Correlation }\end{array}$ & $629^{* *}$ &,$- 370^{* *}$ &,- 051 \\
\hline & $\begin{array}{c}\text { Sig. } \\
\text { (bilateral) }\end{array}$ & ,000 & ,003 & 693 \\
\hline \multirow{2}{*}{$\begin{array}{l}\text { Self-assessment of } \\
\text { linguistic } \\
\text { competence in } \\
\text { Spanish }\end{array}$} & $\begin{array}{c}\text { Pearson } \\
\text { Correlation }\end{array}$ &,$- 280^{*}$ &, $341^{* *}$ &, $345^{* *}$ \\
\hline & $\begin{array}{c}\text { Sig. } \\
\text { (bilateral) }\end{array}$ & 027 & ,007 & ,006 \\
\hline
\end{tabular}

The following summary (Table 3 ) shows the existing correlations, their direction, and significance level.

Table 3. Summary of correlations H3 secondary hypotheses

\begin{tabular}{|l|l|l|l|}
\hline & $\begin{array}{l}\text { ATTITUDES } \\
\text { TOWARDS } \\
\text { CATALAN }\end{array}$ & $\begin{array}{l}\text { ATTITUDES } \\
\text { TOWARUDSS } \\
\text { TOWARDS }\end{array}$ \\
\hline $\begin{array}{l}\text { Competence in } \\
\text { Catalan }\end{array}$ & $\begin{array}{l}\text { Direct correlation } \\
\text { Level } 0.01\end{array}$ & $\begin{array}{l}\text { Indirect Correlation } \\
\text { Level } 0.01\end{array}$ & No correlation \\
\hline $\begin{array}{l}\text { Competence in } \\
\text { Spanish }\end{array}$ & $\begin{array}{l}\text { Indirect Correlation } \\
\text { Level } 0.05\end{array}$ & $\begin{array}{l}\text { Direct correlation } \\
\text { Level } 0.01\end{array}$ & $\begin{array}{l}\text { Direct correlation } \\
\text { Level } 0.01\end{array}$ \\
\hline
\end{tabular}

Hence, it can be concluded that there is a correlation between the self-assessed competence in both the majority and the minority 
languages and the student's attitudes towards both the co-official languages. The results also confirmed the correlation between the self-assessed competence in Spanish and the students' attitudes towards the foreign language. The non-verification of the correlation between the self-perceived competence in Catalan and the students' attitudes towards English, confirmed its null hypothesis.

After confirming a correlation in 5 out of the 6 possible variable combinations, we can conclude that the posed hypothesis is confirmed, and therefore "There is a correlation between the selfassessed competence in L1 and L2 and the students' attitudes towards the analysed languages», wherefrom the following conclusion can be drawn.

There are strong positive correlations between self-assessed competence in each of the co-official languages and attitudes towards these. Thus, a higher self-evaluated competence in Catalan correlates to more positive attitudes towards the minority language and in the same vein there is a powerful association between higher competence in Spanish and more favourable attitudes towards the majority language. On the contrary, linguistic competence and attitudes between the two co-official languages are negatively correlated. Regarding the foreign language, there is a positive correlation between competence in Spanish and attitudes towards English, although this correlation is smaller than previous linear relationships.

From the previous results we can conclude that there is a correlation between self-perceived competence and language attitudes, thus confirming our hypothesis.

\section{Conclusions}

From the analysis and discussion of the results of our study in light of previous research (Lasagabaster 2005a, Huguet 2007a, Lasagabaster 2007, Safont 2007, Portolés 2011, 2015, Lasagabaser 2017, Lasagabaster 2017), several conclusions can be drawn as regards both language policies and future research. Our findings confirm our hypothesis, and therefore, we can corroborate that there is a correlation between self-assessed linguistic competence and the students' attitudes towards the three analysed languages. Thus, the variable self-assessed competence in both the co-official languages yielded significant differences on the attitudes towards the three languages in contact at the same time that proved to have a bearing on students' linguistic attitudes.

As it had been stated in the introduction, there is bidirectional relationship between language attitudes and language proficiency (Lasagabaster 2003)(cited in Portolés 2015). This is confirmed by our results that observe a direct correlation between self-assessed 
competence in Catalan and more favourable attitudes towards the minority language. Similarly, students' positive attitudes towards Spanish are also directly correlated with referring higher competences in the majority language. These findings coincide with the conclusions of previous studies in bilingual and multilingual contexts (Lasagabaster 2003, 2005a, Laugharne 2007, Querol and Huguet 2010, Portolés 2011, Madariaga, Huguet, and Janés 2016).

As we had expected according to previous research (Baker 1992, Lasagabaster 2005b, Huguet 2007a, Huguet, Laprestra, and Madariaga 2008, Lasagabaser 2017, Lasagabaster 2017), the findings yielded notable disparities on language attitudes towards the majority and the minority languages, in this case between the Spanish-speaking and the Catalan-speaking groups. It is important to remark that the attitudes of self-perceived bilinguals tend to converge with those of the students whose mother tongue is only Catalan. Moreover, the study also showed an inverse correlation between respondents' mother tongue and their attitudes towards the other co-official language, be it Catalan or Spanish. Thus, attitudes towards the majority and the minority languages were skewed towards the positive pole or the opposite of the attitudinal continuum according to the positive or negative attitudes displayed towards Catalan or Spanish.

As for the limitations of the study we should refer to the sample, which is one of the most commonly shortcomings encountered in the field of educational research. In fact, the size and localization of the sample does not automatically make the findings fully extensible to the rest of the Valencian Community. For this reason, these constraints on generalizability require additional research to be conducted with extended samples covering an area as extended as possible, to amend possible shortcomings associated with crosssectional studies, longitudinal studies would also be advisable.

The considerable differences in language attitudes towards the three languages present in the curriculum lead us to consider the need of redesigning language and language-in-education policies that allow every student to achieve the same level of bilingualism and to foster positive attitudes towards the three languages.

Further research in this field is essential since drawing a map on language attitudes amongst the students allows to investigate the causes and shift or minimize their negative impact. Affective factors are a paramount element in determining the future downward trend in the use of a minority language since language use and vitality are strongly influenced by the speakers' perception of the languages in contact (Baker 1992). Thus, reverting a negative attitude towards a language not only leads to an increase in the motivation to learn it and use it, but also on the attainment of a higher linguistic command 
which paves the path, in conjunction with other factors, to the possible revitalization of its social use.

It is common knowledge that the official status of a language does not guarantee its survival, nor its inclusion as a subject in the educational system implies a wider use. Therefore, it is essential to promote effective language and language-in-education policies to try to balance the status of the majority and the minority languages with the aim of achieving a balanced bilingualism that boosts positive language attitudes towards the three languages involved.

\section{References}

Alcón, Eva, and Maria Pilar Safont. 2013. «English and multilingualism.» In Encyclopaedia of Applied Linguistics, edited by Carol A. Chapelle, 3883-3888. New York: Wiley \& Sons.

De Angelis, Gessica, and Jean-Marc Dewaele. 2011. New trends in crosslinguistic influence and multilingualism research. Clevedon: Multilingual Matters.

Arnau, Joaquim, and F. Xavier Vila. 2013. "Language in education policies in the Catalan language area." In Reviving Catalan at school Challenges and instructional approaches edited by Joaquim Arnau, 1-28. Bristol: Multilingual Matters.

Auer, Peter, and Li Wei, eds. 2007. Handbook of multilingualism and multilingual communication. Vol. 5. Berlin: Mouton de Gruyter.

Baker, Colin. 1992. Attitudes and language. Clevedon: Multilingual Matters.

Bodoque, Anselm. 2011. "El model valencià de política lingüística.» Revista de Llengua i Dret 56: 143-171.

Bou, Mireia. 2016. "Les actituds lingüístiques i la tria lingüística en les comunicacions formals de nous moviments socials a Catalunya.» Revista de Llengua i Dret 66: 38-61.

Brown, James D. 1988. Understanding research in second language lerning: A teacher's guide to statistics and research design. Cambridge: Cambridge University Press.

Brown, James D., and Theodore S. Rodgers. 2002. Doing second language research: An introduction to the theory and practice of second language research for graduate/master's students in TESOL and applied linguistics, and others. Oxford: Oxford University Press.

Calatayud, Víctor A. 2011. "Análisis de la realidad sociolingüística del valenciano.» Papers: revista de sociología 96 (2): 501-514. 
Cenoz, Jasone. 2013. "Defining multilingualism.» Annual Review of Applied Linguistics 33: 3-18.

Cenoz, Jasone, and Fred Genesse, eds. 1998. Beyond bilingualism: Multilingualism and multilingual education. Vol. 110. Clevedon: Multilingual Matters.

Cenoz, Jasone, and Ulrike Jessner, eds. 2000. English in Europe: The acquisition of a third language. Vol. 19. Clevedon: Multilingual Matters.

Dewaele, Jean-Marc. 2005. "Sociodemographic, psychological and politico-cultural correlates in Flemish students' attitudes towards French and English.» Journal of Multilingual and Multicultural Development 26: 118-137.

Dewey, Martin. 2007. "English as a lingua franca and globalization: an interconnected perspective." International Journal of Applied Linguistics 17 (3): 332.

Dörnyei, Zoltdn. 1990. "Conceptualizing motivation in foreignlanguage learning." Language Learning 40 (1): 45-78.

Dörnyei, Zoltdn. 2003. "Attitudes, orientations and motivations in language learning: Advances in theory, research and applications.» Language Learning 53 (1): 3-32.

Extra, Guus, and Durk Gorter, eds. 2001. The other languages of Europe: Demographic, sociolinguistic, and educational perspectives. Clevedon: Multilingual Matters.

Garrett, Peter. 2001. "Language attitudes and sociolinguistics.» Journal of Sociolinguistics 5 (4): 626-631.

Generalitat Valenciana. 1982. Estatut d'Autonomia de la Comunitat Valenciana.

Generalitat Valenciana. 1983. Llei d'Ús i Ensenyament del Valencià. Llei 4/1983, 23 de novembre.

González-Martínez, Juan L. 2010. «Actitudes lingüísticas en una comunidad rural: Els Ports (Castellón). Datos de un cuestionario sociolingüístico.» Cultura, Lenguaje y Representación 8: 75-95.

Gorter, Durk, ed. 2006. Linguistic landscape: A new approach to multilingualism. Clevedon: Multilingual Matters.

Herdina, Philip, and Ulrike Jessner. 2002. A dynamic model of multilingualism: Perspectives of change in psycholinguistics. Clevedon: Multilingual Matters.

House, Juliane. 2003. «English as a lingua franca: A threat to multilingualism?» Journal of Sociolinguistics 7 (4): 556-578. 
Huguet, Ángel. 2007a. "Language use and language attitudes in Catalonia.» In Multilingualism in European bilingual contexts: Language use and attitudes, edited by David Lasagabaster and Ángel Huget, 17-39. Clevedon: Multilingual Matters.

Huguet, Ángel. 2007b. «Minority languages and curriculum: The case of Spain.» Language, Culture and Curriculum 20 (1): 70-86.

Huguet, Ángel, and Xosé A. González-Riaño. 2004. Actitudes lingüísticas, lengua familiar y enseñanza de la lengua minoritaria. Barcelona: Horsori.

Huguet, Ángel, Cecilio Laprestra, and José M. Madariaga. 2008. «A study on language attitudes towards regional and foreign languages by school children in Aragon, Spain." International Journal of Multilingualism 5 (4): 275-293.

Huguet, Ángel, and Enric Llurda. 2001. "Language attitudes of school children in two Catalan/Spanish bilingual communities.» International Journal of Bilingual Education and Bilingualism 4 (4): 267-282.

Janés, Judit. 2006. "Las actitudes hacia las lenguas y el aprendizaje lingüístico.» Revista Interuniversitaria de Formación del Profesorado 20 (56): 117-132.

Laoire, Muiris Ó. 2007. "Language use and language attitudes in Ireland.» In Multilingualism in Europeanbilingual contexts: Language useand attitudes, edited by David Lasagabaster and Ángel Huguet. Clevedon: Multilingual Matters.

Lasagabaser, David. 2017. "Language Learning Motivation and Language Attitudes in Multilingual Spain from an International Perspective.» Modern Language Journal 101: 583-596.

Lasagabaster, David. 2000. "Three languages and three linguistic models in the Basque educational system." In Bilingual Education and Bilingualism, edited by Jasone Cenoz and Ulrike Jessner, 179-197. Clevedon Multilingual Matters.

Lasagabaster, David. 2003. Trilingüïsmo en la enseñanza. Actitudes hacia la lengua minoritaria, la mayoritaria y la extranjera. Lleida: Editorial Milenio.

Lasagabaster, David. 2005a. "Attitudes towards Basque, Spanish and English: An analysis of the most influential variables." Journal of Multilingual and Multicultural Development 26 (4): 296-316.

Lasagabaster, David. 2005b. "La presencia de tres lenguas en el currículo: Multilingüismo en los contextos canadiense y español.» Revista de Educación 337: 405-426. 
Lasagabaster, David. 2007. "Language use and Language attitudes in the Basque Country." In Multilingualism in European bilingual contexts: Language use and attitudes, edited by David Lasagabaster and Ángel Huguet. Clevedon: Multilingual Matters.

Lasagabaster, David. 2017. "Language Learning Motivation and Language Attitudes in Multilingual Spain from an International Perspective.» Modern Language Journal 101: 583-596.

Lasagabaster, David, and Juan Manuel Sierra. 2009. «Immersion and CLIL in English: more differences than similarities.» ELT Journal 64 (4): 367-375.

Laugharne, Janet. 2007. "Language use and language attitudes in Wales.» In Multilingualism in European bilingual contexts: Language use and attitudes, edited by David Lasagabaster and Ángel Huguet, 208-233. Clevedon: Multilingual Matters.

Madariaga, José M., Ángela Huguet, and Judit Janés. 2016. «Language attitudes in Catalan multilingual classrooms: educational implications.» Language and Intercultural Communication 16 (2): 216-234.

Nightingale, Richard. 2012. Bridging the gap between the internal and the external: The effect of sociocultural factors in adolescent learners' attitudes towards English. Saarbrücken: Lambert Academic Publising.

Nightingale, Richard. 2016. "The effect of out-of-school media contact on language attitudes in multilingual adolescents: A complex psycho-sociolinguistic system.» PhD dissertation. Castelló de la Plana: Universitat Jaume I.

Pascual, Vicent. 2006. El tractament de les llengües en un model d'educació plurilingüe per al sistema educatiu valencià. València: Conselleria de Cultura, Educació i Esport.

Pascual, Vicent. 2011. L'escola valenciana. Un model d'educació plurilingüe $i$ intercultural per al sistema educatiu valencià. Generalitat Valenciana. València: Conselleria de Cultura, Educació i Esport.

Pavlenko, Aneta. 2007. Emotions and multilingualism. Cambridge: Cambridge University Press.

Phillipson, Robert. 2004. English-only Europe? Challenging language policy. London: Routledge.

Phillipson, Robert. 2006. Language policy and linguistic imperialism. An introduction to language policy: Theory and method. Oxford: Blackwell Publishing. 
Phillipson, Robert, and Tove Skutnabb-Kangas. 1996. "English only worldwide or language ecology?» TESOL Quarterly 30 (3): 429452.

Portolés, Laura. 2011. A multilingual portrait of language attitudes in higher education. Saarbrücken: VDM Verlag.

Portolés, Laura. 2014. "Analysing prospective teachers' attitudes towards three languages in two different sociolinguistic and educational settings." In Teaching and learning in multilingual contexts: sociolinguistic and educational perspectives, edited by Agnieszka Otwinowska and Gessica De Angelis, 50-75. Clevedon: Multilingual Matters.

Portolés, Laura. 2015. Multilingualism and very young learners: An analysis of pragmatic awareness and language attitudes. Berlin: Walter de Gruyter.

Querol, Mónica, and Ángel Huguet. 2010. «Conocimiento lingüístico y actitudes lingüísticas. Un estudio sobre sus relaciones en el alumnado de origen inmigrante en Cataluña.» Segundas Lenguas e Inmigración 3: 61-79.

Riley, Philip. 2007. Language, culture and identity: An ethnolinguistic perspective. London: Continuum.

Romaine, Suzanne. 2007. "The impact of language policy on endangered languages." In Democracy and human rights in multicultural societies, edited by Paul de Guchteneire and Matthias Koenig, 217-236. London: Routledge.

Safont, María Pilar. 2007. "Language use and Language attitudes in the Valencian Community." In Multilingualism in European bilingual contexts: Language use and attitudes, edited by David Lasagabaster and Ángel Huguet, 90-116. Clevedon: Multilingual Matters.

Safont, María Pilar. 2015. "The promotion of multilingualism in a Catalan-speaking area. Familial challenges in the Valencian Community.» In The Multilingual Challenge: Cross-Disciplinary Perspectives, edited by Ulrike Jessner and Claire J. Kramsch, 3959. Berlin: Walter de Gruyter.

Todeva, Elka, and Jasone Cenoz, eds. 2009. The multiple realities of multilingualism: Personal narratives and researchers' perspectives. Berlin: Walter de Gruyter.

Torró, Tudi. 2001. «El Català a l'escola del País Valencià: dades i reflexions.» Treballs de Sociolingüística Catalana 20: 193-208. 


\section{Developing plurilingual competences in primary education}

Alicia Chabert

chabert@uji.es 
I. Abstract

\begin{abstract}
I. Abstract
Now more than ever the English language has become a necessity in several aspects of our life. As a consequence of globalisation and the need of an international and intercultural communication tool (Jenkins, Cogo, and Dewey 2011, Cogo 2012), English has emerged as a lingua franca and English acquisition is in the spotlight. This research aims to understand how English teaching could be improved at school. While different language approaches and methodologies are used, the mother tongue of the learner is not taken into account in English teaching and the CEFR (Common European Framework of Reference for Languages) is still used as guideline, not only in Europe but also worldwide (Nakatani 2012). We live in a multilingual world where all languages are in constant contact and identities are blurred, mostly when English has raised as the universal language. Taking this into account, our research focuses on a plurilingual approach to English language teaching in order to improve English learning in primary school based on an understanding of languages from an ecological point of view. The study is conducted in five primary school centres, in 3 different countries.
\end{abstract}

Keywords: plurilingualism, multilingualism, linguistic ecology, lingua franca, plurilingual competence, foreign language acquisition.

\title{
II. Context of this research and literature review
}

\section{A dichotomy: English as a lingua franca and multilingualism}

English is the dominant language of the $21^{\text {st }}$ century, with 379 million native speakers (Statista 2019) and approximately 1.75 billion users, which represents approximately a quarter of the world's population (British 2013). The British Council estimates that there are 2 billion people using English in their daily life, or at least learning to use it. This increase in English language speakers is one of the consequences of globalisation, along with the rapid evolution of technology (telephones, the media, internet...) and it is intrinsically linked to English becoming a lingua franca (ELF).

Firth (1996) first defined ELF as a "contact language» between people with different mother tongues and who chose English as their foreign language of communication. However, most researchers now believe native speakers should be included in the ELF paradigm (Friedrich and Matsuda 2010, Jenkins, Cogo, and Dewey 2011, Seidlhofer 2011). Seidlhofer (2005) included native speakers of 
English for whom ELF is also an additional language, understanding ELF as intercultural communication. We understand ELF as the best approach to English learning in a multilingual society taking that the language is taught without associating it necessarily to a specific culture. In this vein, House (2003) distinguishes between 'languages for communication' and 'languages for identification'.

In this moment in history, with immigration, ease to travel, social networks and the internet, among other reasons, the lines between languages in use and identities are blurred and constantly changing. Cultural and linguistic diversity, multilingualism and plurilingualism are among the key values of Europe, however the guidelines for English teaching keep having a monolingual approach, which contradicts its own recommendations for plurilingualism and multilingualism. The Council of Europe makes a distinction between the linguistic diversity of a geographical region, which is referred to as multilingualism, and the linguistic competence of an individual, also known as plurilingualism. However, both terms are usually interchanged and most of the time we will find that the most common term is multilingualism, referring to the linguistic diversity of both individual and geographical region. We will, nevertheless, keep the distinction between the two.

The Common European Framework of Reference for Languages: learning, teaching, assessment (Council of Europe 2001 - henceforth CEFR) is the current language guidelines used not only in Europe but also worldwide. It was originally designed to describe the language learners' abilities at different stages of their learning and to be language-neutral, so it could be applied to any foreign language learning situation. Because of its neutral nature, this framework does not take into account the different mother tongues the learners have, which impact the strengths and weaknesses of each student, so "the practice of applying the CEFR has mostly neglected the dimension on plurilingualism and plurilingual competence» (Kalliokoski 2011, 87). Nevertheless, plurilingualism has now become an unavoidable factor to take into account in most research and it has been introduced in the CEFR. Plurilingualism is presented in the CEFR as a changing competence, in which the learner's resources in one language or variety may be very different in nature to those in another (Council of Europe 2018), However the monolingual immersive approach to foreign language teaching is still recommended.

\section{Monolingual and plurilingual approaches to foreign language acquisition}

In the field of English language teaching, the monolingual approach has been appointed as the official approach to be applied in English classes. Even though, literature and research suggests that 
it has been a questionable approach for years and that a plurilingual approach could be more beneficial for English learning.

For decades, there has been a main focus on major exposure to L2 and the importance of a native speaker teacher in the English classroom. In fact, while the perception in some countries is changing, in many others the non-native English teachers are still positioned as deficient speakers of the language, with imperfect knowledge of the foreign language and culture (Walkinshaw and Oanh 2014). Regardless of the nativeness of the teacher, the use of the mother tongue in the classroom has been very controversial for decades. From Selinker's (1972) definition and understanding of interlanguage as a separate linguistic system in which the learner fossilizes errors due to similarities with the native language and this prevents the person from achieving a full level of facility in the use of the target language, many theories were introduced on how the first language (L1) influenced the second language (L2) learning negatively. As Selinker, many researchers supported this view of L1 being a detrimental 'interference' to L2 learning (Lado 1957, James 1980, Flege and Davidian 1984, Yu 2009). Nevertheless, soon enough scholars started proving how the $\mathrm{L} 1$ could be used as an instrumental tool to favour L2 learning (Cook, Long, and McDonough 1979, Wells 1999, Cook 2001, Lasagabaster 2001, Herdina and Jessner 2002, Jessner, De Angelis, and Kresic 2015), and slowly but surely there has been a recent shift towards this approach in which mother tongues are positive to subsequent language learning. This research shift has focused on using languages to learn other languages, i.e. developing the plurilingual competences of the students to use languages as a strategic competence. The Council of Europe refers to this competence as the repertoire of resources which the students acquire in all the languages they know or have learned, and the cultures associated with them (Beacco et al. 2016). So much evidence has been provided in literature that whether using L1 on L2 learning is not the focus of the debate anymore, but how to use L1 in a way that will be beneficial to second language learning.

Despite plurilingualism being recommended by the Council of Europe and by linguistic specialists, the common belief of the English-only classroom still prevails in teachers and parents of students. Europe has been promoting linguistic diversity and general guidelines on plurilingualism without real application, which has resulted in confusion and programmes that claim to be plurilingual but in which a plurilingual approach is still lacking, as it can be observed in the Plurilingual Plan in the Valencian Region.

The Plurilingual Plan is a new program that has been implemented in the past couple of years in the Valencian Region and involves the division of subjects by language so that the students are exposed to three languages in different contexts: Spanish, Catalan 
and English. The goal of this program is to maximise the communicative competence in different languages by creating contexts in which those languages are to be used. However, while this program focuses on increasing the opportunities to use a certain language, it is still focused on a monolingual approach since it does not allow more than one language to be used in a specific setting and keeps all languages as separate units understanding them as if they do not interact with each other. Even though this programme claims to be plurilingual, it fails to use a plurilingual approach.

\section{Objectives of our study}

Our research aims to describe how the English language is spreading in our multilingual reality and analyse how the current language policies and guidelines are implemented. At the same time, we propose a plurilingual approach that is tested in different countries with the purpose of demonstrating how English teaching could be improved taking into account the mother tongue of the students and using an ecological linguistic approach, so English is learnt as a communicative tool and not an identification instrument that devalues different cultures and languages.

The ecology of language is a metaphor first used by Haugen (1972) to describe the relationships among the diverse forms of language in the world and the groups of people who use them. We will use this concept as part of the approach we propose, that is, a plurilingual approach that values each language for the learning of a subsequent language. We consider essential that students gain knowledge and understanding of different languages so that they develop social and intercultural understanding, but in order to achieve this, plurilingualism needs to be promoted in the classroom. Despite the different views on cross-linguistic influence, it has been demonstrated that the interaction among language systems in a plurilingual speaker develops a high level of awareness of languages (Jessner 2006). In our international study we aim to demonstrate how the interaction of languages facilitates the learning in a subsequent language.

To put simply, our research objectives are as follows:

- Comparing how the mother tongue influences English learning differently in each country (Norway, China and Spain participate in this research).

- Arguing that, if the mother tongue influences learning English, the teaching methodology should be adapted accordingly.

- Demonstrating that a plurilingual approach is always more beneficial than a monolingual one in a primary school setting. 
- Demonstrating that using an ecological linguistic approach while learning English will provide the students with the understanding of English as an international communication tool separate to the value of the mother tongue a an identification tool. In Seidlhofer's words, we need to change the monolingual view of English as a property of its native speakers "to the recognition of ELF as an appropriated communicative resource, its use characterized by continuous negotiation of meaning and linguistic adaptation and plurality».

In order to meet our objectives, we are carrying out an international study that includes speakers of a roman language (Spanish), Scandinavian language (Norwegian), and Asian language (Chinese). This study focuses on the learning of primary school students and aims to understand their attitudes towards English and their mother tongue, as well as the objectives above stated.

\section{Research design: method and participants}

Five centres participate in this study: three in the Valencian Region, one in Norway and one in China. While our initial proposal was to have the same amount of schools in every country, due to the lack of response and willingness to participate we were only able to secure one in Norway and one in China, in comparison to the three we have in the Valencian Region. This issue will be included in the limitations of our study.

In terms of participants, we focused on the $6^{\text {th }}$ year of Primary School and the groups include between 20 and 30 students (this is specified in each segment of the experiment). As for methodology, we use experimental research along with survey, so we use a mix of quantitative and qualitative approaches. The process has been carried out as follows:

- 2016 to 2017 - Literature review and research.

- 2017 to 2018 - Surveys to parents and students, pilot test and interviews to teachers.

- 2018 to 2019 - Full study on the three countries participating in the study, questionnaires to the students and analysis of all obtained results.

All the results will be analysed within the current frameworks and guidelines, as well as the countries linguistic and education policies. We will also compare the different policies between Europe and Asia (ASEAN+3 countries $^{1}$ ).

In 2017 we carried out the pilot study, for which we used a monolingual approach in the control group and a plurilingual

\footnotetext{
${ }^{1}$ Association of Southeast Asian Nations including Indonesia, Malaysia, Philippines, Singapore, Thailand, Brunei, Myanmar, Cambodia, Laos and Vietnam, plus China, South Korea and Japan
} 
approach in the experimental group. In order to test the experiment in different countries, we conducted the study in one of the centres participating from Spain and the centre participating in Norway. Before carrying out the study we interviewed the teachers to learn about the students' background, their general behaviour and the style used in the classroom. Afterwards, we chose a topic and grammar concept that coincided in both books (Norwegian and Spanish) and the students had not yet studied, which in this case was the past continuous. For the monolingual approach we used only English for instruction, explanation and communication. On the other hand, for the plurilingual approach we used the mother tongue of the students when needed for instruction and we compared English and the mother tongue when explaining grammar concepts (in Norway we received help of the Norwegian teacher in the classroom for this purpose). After this we examined the students using exercises based on the new concepts and grammar learnt. The exercises used as a test included three activities: fill the gaps activity (comprehension-production), identification of concept in a reading activity (comprehension) and creation of sentences using the concept explained in class (production). In both countries we obtained better results using a plurilingual approach (see Chabert 2017).

Surveys were carried out in the classroom focusing on whether the students received any English tutoring after school, the amount of time they spent watching TV or movies in English, their feelings about their mother tongue and English and their opinion on whether they believe they learn better when the class is taught fully in English or in English and their mother tongue.

Based on this first process, we realised that it would be very interesting to include an oral part of the activity and analyse the plurilingual competence in speaking as well as writing. For this reason, in the main study we modified the approach as follows:

- Two sessions are held in each group (control and experimental group per centre).

- In the first session, a new concept is explained and practised. At the end of the class the students do the test about the specific subject (as with the previous pilot test, in one class we use a plurilingual approach and monolingual in the other).

- In the second class we review the concept explained in the first class and record the audio of the whole class. A role play activity is carried out in couples.

- This time the concept and unit chosen to be explained and used in the test were: the imperative, giving and receiving directions and prepositions of place and movement. 
- After the activities, students were requested to fill in a questionnaire to gather information about their backgrounds and their affective filters when learning English.

In terms of the activities to be analysed in this study, we included a comprehension activity, a fill the gaps and a production exercise. As for the role play: the students are partnered in couples. One of them acts as him/herself, the teacher acts as a foreigner who is lost and the second student is the grandparent of the student. The students enact a situation in which they are going to the market and encounter a lost foreigner that is asking for directions. The foreigner (who, for the purpose is not a native English Speaker) asks for help to the grandparent but he/she does not speak English, so the student acting as him/herself will intervene. This activity is done in both experimental and control groups, however in the experimental group the student is allowed to use a little Spanish if they get stuck (up to 3 words). The specific situation of this role play is purposeful, so the foreigner is in this case a German speaker, so she does not use English only. The reason for this is to show that it is okay to use a different language to express yourself if the English terminology is not readily available and also to see that we can draw from other languages to understand other languages (similarities between languages). On the other hand, by bringing a guardian figure (grandparent/parent) and actually using language to help them motivates the students, gives them a sense of autonomy and relevance. This fact is also then tested after the activity by asking the students about what they would do in this situation. In the experimental group, the students are allowed to use a little of their mother tongue to show them that is better to say something in their mother tongue than getting stuck or being scared of saying something wrong. By audio recording this, we analyse if the fluency is affected when the option of using a few words in their mother tongue is given.

At the moment we have the results of the pilot test and two of the schools in Spain. The study in the third school is programmed to start in February 2019, the study in Norway will take place in January 2019 and in China in April 2019. Once all the results are available we will analyse them individually and compare them, taking the current educational guidelines of the relevant country into account.

\section{Results and expectations}

During the pilot test we achieved better results in all activities and both countries when using a plurilingual approach. See below figure 1 for reference: 


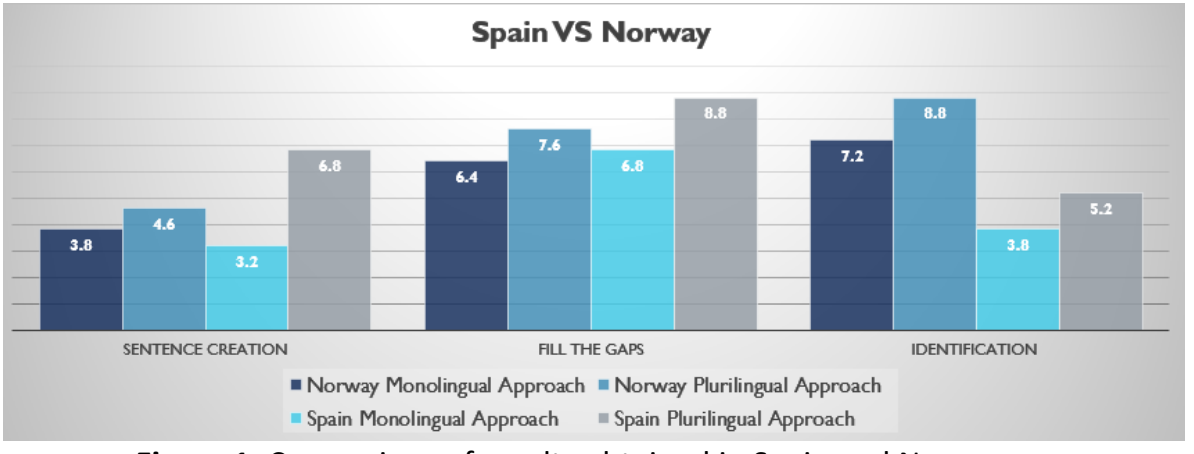

Figure 1. Comparison of results obtained in Spain and Norway

Also in the first test, we questionnaires were administered to parents regarding their preference on language use in the classroom and, interestingly, $100 \%$ of the parents in the Spanish school believed only English should be used in the classroom, whereas $97.3 \%$ of the parents in the Norwegian school believed both Norwegian and English should be used in the classroom. We found this information relevant taking into account that Norway has a very high level of proficiency (see EF EPI index ${ }^{2}$ ) in English as compared to Spain, and yet they use their mother in their classes.

Regarding the current main study, based on the pilot study and the first results obtained in the two schools already tested, we expect students to achieve better results in the activities when both English and their mother tongue are used in the classroom. We further expect students to be more confident in English when the use of their mother tongue when needed is not punished, as per the results on the first schools, and we believe the role play activity will provide student with a sense of autonomy, accomplishment and positive attitude towards languages (including their mother tongue).

\section{Conclusion and limitations}

As above mentioned, based on the results of the pilot study, and the first results obtained, a plurilingual approach is shown to achieve better results in English learning. Our study supports the theory that the $L 1$ is deeply involved in subsequent language learning and that fostering plurilingual competences will teach the students to use L1 in a strategic way to navigate the voids in $L 2$ knowledge and progress in it accordingly. In this way, teaching all languages as part of a communication system would develop strategic competences that would facilitate learning in any language. Also, by teaching English from an ecological perspective in which English is not associated with prestige but with international and intercultural communication between people in a globalised world, the preservation of cultures and languages would thrive.

\footnotetext{
${ }^{2}$ https://www.ef.com/wwen/epi/
} 
It is important to note, as part of our limitations, that all variables in this international study need to be considered in terms of: mother tongue, socio-economic background, bilingualism of the students, rural vs. urban area, and tutoring outside school. Also, further testing (from a longitudinal perspective) would be needed to check the impact of the long-term application of a plurilingual approach. Nevertheless, this study significant in promoting and examining the concept of plurilingual competence as established by the Council of Europe, which is believed to be necessary to accompany linguistic policies (Coste, Moore, and Zarate 2009).

\section{References}

Beacco, Jean-Claude, Michael Byram, Marisa Cavalli, Daniel Coste, Mirjam Egli Cuenat, Francis Goullier, and Johanna Panthier. 2016. Guide for the Development and Implementation of Curricula for Plurilingual and Intercultural Education. Brussels: Council of Europe.

British, Council. 2013. The English Effect. The impact of English, what it's worth to the UK and why it matters to the world. London: The British Council.

Chabert, Alicia. 2017. "La influència de la llengua materna en l'aprenentatge d'anglés arreu del món.» Fòrum de Recerca 22.

Cogo, Alessia. 2012. «English as a Lingua Franca: concepts, use, and implications.» ELT Journal 66 (1): 97-105. doi: 10.1093/elt/ccr069.

Cook, Vivian. 2001. "Using the First Language in the Classroom.» The Canadian Modern Language Review 57 (3): 402-423. doi: 10.3138/cmlr.57.3.402.

Cook, Vivian J., J. Long, and Steven McDonough. 1979. «First and second language learning." In The Mother Tongue and Other Languages in Education, edited by George E. Perren. London: Centre for Information on Language Teaching.

Coste, Daniel, Danièle Moore, and Geneviève Zarate. 2009. Plurilingual and Pluricultural Competence - Studies towards a Common European Framework of Reference for language learning and teaching. Brussels: Council of Europe.

Council of Europe. 2018. Common European Framework of Reference for Languages: Learning, Teaching, Assessment Companion Volume with New Descriptors. Brussels: Language Policy Programme.

Firth, Alan. 1996. "The discursive accomplishment of normality: On 'lingua franca' English and conversation analysis.» Journal of 
Pragmatics 26 (2): 237-259. doi: 10.1016/0378-2166(96)00014-

8.

Flege, James Emil, and Richard D. Davidian. 1984. "Transfer and developmental processes in adult foreign language speech production." Applied Psycholinguistics 5 (4): 323-347. doi: 10.1017/S014271640000521X.

Friedrich, Patricia, and Aya Matsuda. 2010. «When Five Words Are Not Enough: A Conceptual and Terminological Discussion of English as a Lingua Franca.» International Multilingual Research Journal 4 (1): 20-30. doi: 10.1080/19313150903500978.

Haugen, Einar. 1972. The ecology of language. Stanford: Stanford University Press.

Herdina, Philip, and Ulrike Jessner. 2002. A Dynamic model of multilingualism: perspectives of change in psycholinguistics. Clevedon: Multilingual Matters.

House, Juliane. 2003. "English as a lingua franca: A threat to multilingualism?» Journal of Sociolinguistics 7 (4): 556-578.

James, Carl. 1980. Contrastive analysis. London: Longman.

Jenkins, Jennifer, Alessia Cogo, and Martin Dewey. 2011. «Review of developments in research into English as a lingua franca.» Language Teaching 44 (3): 281-315. doi: 10.1017/S0261444811000115.

Jessner, Ulrike. 2006. Linguistic Awareness in Multilinguals: English as a Third Language. Edinburgh: Edinburgh University Press.

Jessner, Ulrike, Gessica De Angelis, and Marijana Kresic. 2015. Crosslinguistic influence and crosslinguistic interaction in multilingual language learning. New York: Bloomsbury Academic.

Kalliokoski, Jyrki. 2011. «Plurilingual competence, Styles and Variation.» Journal of Estonian and Finno-Ugric Linguistics 2 (2): 87-110.

Lado, Robert. 1957. Linguistics across cultures; applied linguistics for language teachers. Ann Arbor: University of Michigan Press.

Lasagabaster, David. 2001. "The Effect of Knowledge About the L1 on Foreign Language Skills and Grammar.» International Journal of Bilingual Education and Bilingualism 4 (5): 310-331. doi: 10.1080/13670050108667735.

Nakatani, Yasuo. 2012. «Exploring the Implementation of the CEFR in Asian Contexts: Focus on Communication Strategies.» Procedia.Social and Behavioral Sciences 46: 771-775. doi: 10.1016/j.sbspro.2012.05.196. 
Seidlhofer, Barbara. 2005. "English as a lingua franca.» ELT Journal 59 (4): 339-341. doi: 10.1093/elt/cci064.

Seidlhofer, Barbara. 2011. Understanding English as a Lingua Franca. Oxford: Oxford University Press.

Selinker, Larry. 1972. "Interlanguage.» International Review of Applied Linguistics in Language Teaching 10 (1-4): 209-232. doi: 10.1515/iral.1972.10.1-4.209.

Statista. 2019. The most spoken languages worldwide (native speakers in millions). Hamburg.

https://www.statista.com/statistics/266808/the-most-spokenlanguages-worldwide/.

Walkinshaw, lan, and Duongthi Hoang Oanh. 2014. "Native and NonNative English Language Teachers.» SAGE Open 4 (2). doi: $10.1177 / 2158244014534451$.

Wells, Gordon. 1999. "Using L1 to Master L2: A Response to Anton and DiCamilla's "Socio-Cognitive Functions of L1 Collaborative Interaction in the L2".» The Modern Language Journal 83 (2): 248-254. doi: 10.1111/0026-7902.00019 Cit.

$\mathrm{Yu}$, Xiaoping. 2009. "The Negative Transfer of Mother Tongue in Non-English Majors' Compositions from the Perspective of Syntax.» Asian Social Science 5 (9): 137-141. 


\section{Estilos comunicativos y elaboración hipotáctica:}

implicaciones en los discursos generalistas y especializados

Rosana Moya Isach rosana.moya@uji.es 
I. Resumen

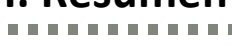

El objetivo de este artículo es analizar la diversidad de los estilos periodísticos de los dos modelos comunicativos de la prensa española - generalista y especializado-, partiendo del estudio de la elaboración hipotáctica de la lingüística sistémico-funcional, que se manifiesta en las oraciones de relativo explicativas. Por medio de un programa de concordancias, se analizará un corpus anotado sintácticamente con el fin de esclarecer las divergencias de los textos periodísticos de información general y especializado (de corte económico y deportivo) para así confeccionar una caracterización definitoria del estilo del género de la noticia de cada modelo, a partir de la función comunicativa de las construcciones explicativas.

Palabras clave: estilos periodísticos, información generalista, información especializada, relativas explicativas, relaciones lógicosemánticas, elaboración hipotáctica, lingüística sistémica.

\section{Introducción}

Las diferencias entre los periódicos generalistas y los especializados son evidentes en todos los aspectos: desde la temática a las audiencias, pasando por la línea editorial, el formato, la formación del periodista, el objetivo de sus noticias, los actores que intervienen en ellas o los valores empresariales. Además, no son nimias las diferencias existentes dentro del patrón de prensa especializada: no es lo mismo hablar de deportes que de economía. Todos estos aspectos influyen de manera decisiva en el uso del lenguaje $y$, por ende, en el estilo comunicativo de cada medio, entendido este como "diversidad de estilos» (Chillón, 2001) o "selección de variedades» (Van Dijk, 1990; Halliday, 1985). La bibliografía ha recogido posturas contrapuestas al respecto. Algunos autores recalcan que los periódicos especializados se permiten la licencia de usar tecnicismos relacionados con su área, mientras que en los de información general predomina un lenguaje más accesible para el «lector común» (Borrat, 1993: 80). Sin embargo, otros opinan que el lenguaje accesible se le debe exigir al periódico especializado, el cual debe llevar a cabo "una estrategia de acercamiento al lector» (Pano, 2012: 777). Así lo matiza, por ejemplo, Del Río (2010: 101102), refiriéndose a la prensa económica:

Una de las máximas del periodismo es que la información se escriba con claridad y de manera directa para que alcance al mayor número de gente posible, y la información económica no es ajena a esta norma. El público que demanda noticias económicas, al margen de que se divulguen en medios generalistas 0 
especializados, exige que sean inteligibles. Se les pide un lenguaje accesible para la ciudadanía en general y que no vaya dirigido solamente a expertos, ya que la información económica, por afectar directamente al bolsillo de los consumidores, despierta una gran sensibilidad en los mismos.

En este sentido, los periódicos especializados en economía concentran diferentes grados de especialización entre sus contenidos, ya que, aunque el perfil mayoritario de público al que va dirigida esta información sea un lector experto, cada vez más hay artículos para un público más variado. Así pues, el lenguaje utilizado por los periodistas está «condicionado por la cantidad de información compartida entre emisor y receptor, pero también por la finalidad del texto» (Martínez, 2010: 712).

Con respecto a la prensa deportiva, la gran cantidad de lectores que registra es una muestra de su elevado consumo, algo que tiene que ver con uno de sus principales objetivos: además de informar, los textos periodísticos deportivos también tienen la función de entretener. Evidentemente, esa rutina se traduce en un lenguaje que adquiere todo su valor por el propósito esencial del medio (que es, al fin y al cabo, una empresa que vende un producto de consumo): el «infotainment» (Gómez, 2014). Esta tendencia a la información y al entretenimiento "hace que en este tipo de prensa especializada cobre más valor la anécdota, la extravagancia, la vida privada de los actores del deporte y las diferencias entre ellos que el esfuerzo de estos, la propia competición y los valores educativos del deporte» (Gómez, 2014: en línea). De este modo, Naranjo (2011: 64) advierte que el lenguaje debe adecuarse a las expectativas del lector: "la audiencia espera una explicación de lo que transcurre en el evento deportivo; una explicación que sea sencilla e inteligible, pero, al mismo tiempo, que resulte lo más completa posible».

En definitiva, el estilo periodístico tiene relación con la función comunicativa de cada medio, de manera que consideramos que estudiar el lenguaje de la prensa (en este caso, la «fisonomía expresiva» y las "aptitudes comunicativas» (Chillón, 2001) de las construcciones de relativo explicativas) aportará datos que nos servirán para confeccionar una caracterización definitoria del estilo del género de la noticia en cada modelo.

\section{Concepto y elementos de análisis}

Este trabajo pretende, pues, estudiar los estilos de dos modelos comunicativos de la prensa española - generalista y especializado-, tomando como base de análisis la lingüística sistémico-funcional y su propuesta de la elaboración hipotáctica, enmarcada en las relaciones 
lógico-semánticas ${ }^{1}$ (Downing y Locke 1992; Halliday y Hassan, 1976;

Halliday y Mathiessen, 2014). En el procedimiento de la elaboración, una oración o un fragmento de texto desarrolla o reelabora otra oración o fragmento, aportando una explicación, una aclaración, un ejemplo o una descripción. Se presenta con oraciones introducidas por conectores (serían relaciones paratácticas) y con cláusulas subordinadas de relativo explicativas (relaciones hipotácticas). Estas últimas constituyen la base de este trabajo ${ }^{2}$.

En su definición de la elaboración hipotáctica, Halliday y Mathiessen (2014: 462) especifican que se trata de una estrategia para introducir el discurso informativo de fondo (discourse background information): una caracterización, una interpretación de algún aspecto de la cláusula dominante o una forma de evaluación. El mecanismo sintáctico de la elaboración hipotáctica es la oración subordinada de relativo explicativa: ${ }^{3}$

Hypotactic elaboration has evolved a special clausal construction that of the non-defining relative clause, with an obligatory anaphoric reference item, the relative, in finite clauses (...). The combination of elaboration with hypotaxis gives the category of non-defining relative clause (also called 'non-restrictive', 'descriptive'). (Halliday y Mathiessen, 2014: 462-464).

La oración de relativo explicativa, también llamada apositiva o incidental por asimilarse a las aposiciones (NGLE, 2009: § 44.4a), por su naturaleza parentética, como recalca la NGLE (2009: § 44.4f), forma un grupo fónico propio que se refleja en la escritura por medio de signos de puntuación cuyo fin es separar la subordinada de su antecedente, y que representan la pausa fónica. Entre esos signos de puntuación está principalmente la coma, aunque de manera esporádica también aparecen el paréntesis y la raya, para marcar gráficamente la naturaleza incidental de la información. Esta particularidad prosódica es la norma general, pese a que también hay excepciones diversas:

a) No siempre se hace pausa ante la relativa explicativa, aunque existen razones semánticas que justifican esa opción en tales casos (NGLE, 2009: § 44.6a). Por ejemplo:

\footnotetext{
${ }^{1}$ Las relaciones lógico-semánticas pueden ser de dos tipos: de expansión o de proyección. Las relaciones de expansión son de elaboración, extensión y ampliación, mientras que las de proyección hacen referencia a los verbos de pensamiento y de comunicación. Todas ellas se manifiestan también desde el punto de vista sintáctico en relaciones paratácticas (coordinación en la gramática tradicional) o hipotácticas (subordinación en la gramática tradicional).

${ }^{2}$ González (2003) valida la aplicación de esta teoría lingüística en su artículo sobre las relaciones lógico-semánticas en las noticias de los periódicos ingleses. Por su parte, Moya (2018) trata el mecanismo de la elaboración paratáctica en la prensa española.

3 Para conocer el estado de la cuestión acerca de la tipología de las oraciones de relativo (especificativas vs. explicativas), consultar Company Rico (2015).
} 
1. Por su parte, "School Spirits» emite un nuevo episodio en el que

el canal se acerca hasta colegios y universidades para conocer las historias sobre espíritus y cuenta con alumnos y profesores de cada centro para narrar sus experiencias (R. C., «Xplora, a la caza de sucesos paranormales emblemáticos», La Razón, 1-3-2014, 85).

b) Las relativas especificativas no siempre sirven para restringir la denotación del grupo nominal, ya que pueden aportar cierta información valorativa; es decir, en ocasiones, las diferencias semánticas entre una misma oración con pausa o sin ella se difuminan (NGLE, 2009: § 44.6b). En este sentido, el papel de las especificativas puede ser más determinativo que restrictivo en casos como este (NGLE, 2009: § 44.5d):

2. Un hombre que se ha identificado como un miembro de la flota rusa del mar Negro ha declarado a Reuters: "Estamos aquí para que no se repita lo que pasó con el Maidán". (Pilar Bonet, "La tensión militar se dispara en Crimea», El País, 1-3-2014, 2).

c) En algunos casos concretos, las especificativas también pueden llevar pausa. Gutiérrez (2004: 228) señala que el que, la que, los que y las que se usan en proposiciones especificativas entre comas:

3. La opción de Phoenix Suns, la que más ha sonado, parece que ha perdido fuerza. (J. I. P, «Pau: "Si me traspasan, sería duro"», As, 202-2014, 33).

Sobre estas, la NGLE (2009: § 44.10l) destaca que algunos autores las denominan oraciones relativas "pseudoapositivas» y se emplean para rectificar, atenuar o matizar el contenido expresado por el antecedente. Como no añaden información nueva, admiten la interpretación restrictiva, de modo que, aunque presenten la pauta prosódica de las explicativas, las considera relativas libres.

d) En otros casos, la oración de relativo puede aparecer en el interior de un grupo nominal en aposición que reproduce el contenido de algún elemento de la oración anterior, a modo de "etiqueta discursiva» (López, 2013) o «encapsulador anafórico» (Borreguero, 2018) ${ }^{4}$. Aunque estas oraciones aparezcan delimitadas por comas, son especificativas (NGLE, 2009: $\S 44.8 \mathrm{~h}$ ), en las que el antecedente se interpreta con valor atributivo:

Fuentes del consejo han relacionado esta propuesta con un hipotético interés de Pemex en segregar la compañía, cosa que la

\footnotetext{
${ }^{4}$ Borreguero (2018: 179) los define como «sintagmas nominales con un componente anafórico que reifican y recategorizan fragmentos discursivos para convertirlos en puntos de anclaje informativos (temas) a partir de los cuales poder desarrollar el discurso».
} 
firma mexicana ha negado. (Miguel Jiménez, «Repsol blindará su españolidad en la junta», El País, 28-2-2014, 27).

Al margen de las excepciones, lo que sí queda claro en los estudios de los gramáticos (y así lo recoge la NGLE) es que hay ciertas normas para el uso de unos relativos $u$ otros, dependiendo del tipo de oración de relativo de que se trate. Así pues, los pronombres relativos que, el/la cual, los/las cuales y quien, así como sus variantes con artículo y con preposición, pueden introducir tanto explicativas y especificativas, con matices. Con los dos primeros pronombres, solo las relativas explicativas admiten antecedentes oracionales $y$, si se trata de relativos complejos, van introducidos por el artículo neutro lo:

5. El director general para España no descarta que Emirates introduzca el mítico Airbus 380 en Barajas en los próximos tres años, lo que implicaría elevar un $20 \%$ la capacidad en asientos ofertada por los dos aviones habilitados para el servicio MadridDubái.(J. F. Magariño, «Emirates reclama cielos abiertos para enlazar España y Latinoamérica», Cinco Días, 18-3-2014, 6).

Que se refiere tanto a personas como a cosas. Cuando se trata de antecedentes muy especificados (como nombres propios $y$ demostrativos), introduce oraciones explicativas entre pausas. Las variantes con artículo y preposición pueden introducir tanto especificativas como explicativas, pero el que, la que, los que y las que únicamente introducen especificativas (incluso con pausa, como ya hemos señalado) o relativas libres, según la interpretación que se adopte. El/la cual y los/las cuales también tienen como antecedente personas o cosas y solo pueden aparecer en explicativas. En el caso de quien, cuyo antecedente se refiere a personas, la variante sin preposición también introduce oraciones explicativas; sin embargo, como señala Rodríguez (2013: 145), «la admisión de su uso en oraciones relativas especificativas o restrictivas se produce cuando una preposición se antepone a las citadas formas pronominales (con el cual, de la cual, en los cuales, mediante las cuales, para quien, por quienes, etc.)».

Por otro lado, el determinante cuyo también puede introducir explicativas. No obstante, no existen relativas explicativas introducidas por cuanto, "ya que, al ser las explicativas estructuras incidentales, se rompería la correlación entre el cuantificador del antecedente y el de la subordinada.» (NGLE, 2009: § 22.6I).

En cuanto a los adverbios relativos donde y cuando, pueden introducir relativas explicativas. En el primer caso, donde y sus variantes con preposición pueden introducir tanto especificativas como explicativas. Cuando también puede introducir los dos tipos de relativas, pero si se trata de antecedentes en forma de adverbios 
pronominales que expresan momento o intervalo temporal, solo introduce explicativas:

6. Ahora el PP sufre un enorme desgaste y lograría el $31,5 \%$ de los votos, 13,1 puntos menos que hace tres años, y el PSOE el 32\%, 3,3 puntos más que entonces, cuando tuvo el peor resultado de su historia. Hay una ligera ventaja de los socialistas. (Fernando Garea, «PP y PSOE, pendientes de movilizar a sus votantes desencantados», El País, 9-3-2014, 26).

En el caso de antecedentes temporales no adverbiales (fechas, por ejemplo), la NGLE da cuenta de que las construcciones con cuando, las que aparecen en incisos que aclaran la referencia de una expresión temporal, tienen rasgos de las relativas explicativas, pero también de las relativas libres ${ }^{5}$ en aposición, puesto que existe la posibilidad de permutar los miembros de la construcción. Más allá de esta discusión, en este análisis consideramos, sin embargo, que se trata de circunstanciales temporales muy marcados que aportan información relevante al antecedente.

Con el caso del adverbio relativo como, también existe cierta controversia al respecto, así como dos interpretaciones. Por un lado, tal como se hace eco la NGLE, hay gramáticos que consideran al relativo como entre incisos como una oración de relativo explicativa. Sin embargo, otros entienden que se trata de un adjunto en aposición, cuya estructura sería como la de las relativas libres. En este sentido, la NGLE apoya esta segunda idea, puesto que «una relativa explicativa no puede aparecer delante de su antecedente» (2009: $\S 44.8 \mathrm{~m}$ ). De esta forma, la prueba de que se trata de una relativa libre es el cambio de orden en oraciones como esta:

7. Faltaba hacer cuadrar, como contestaron desde Cultura a este periódico hace semanas, "las agendas de los altos cargos". (Tommaso Koch, "Luz al final del túnel del Arqueológico», El País, 17-03-14).

En este caso, podríamos decir, sin que haya un cambio semántico con respecto al anterior ejemplo, lo siguiente: "Como contestaron desde Cultura a este periódico hace semanas, faltaba hacer cuadrar las agendas de los altos cargos». Igual que en este ejemplo, el uso del pronombre relativo como entre incisos es habitual para introducir palabras de otros en el discurso (el discurso reportado), algo elemental en el texto periodístico, porque aporta evidencialidad, «noción que designa el compromiso con la veracidad de la información transmitida o con la fuente de la que procede» (NGLE, 2009: § 22.11h). Por tanto, consideramos que analizar el uso

\footnotetext{
${ }^{5}$ La NGLE considera que las relativas libres son una subclase de las relativas especificativas.
} 
de este adverbio relativo puede arrojar datos interesantes referentes al estilo directo e indirecto en el discurso informativo.

En la elaboración paratáctica, la exposición y la ejemplificación contribuían a la divulgación de los contenidos y a la didacticidad, mientras que la clarificación tenía que ver con la progresión informativa y el dinamismo discursivo. Los tres elementos paratácticos influían, además, en el grado de formalidad y en la modalización del texto (Moya, 2018). En la elaboración hipotáctica, las relativas explicativas aportan información en tres vertientes. Como señala Rodríguez (2013: 147), pueden funcionar como un inciso, aclarando algún aspecto del antecedente; pueden aportar información de carácter temático y, por tanto, accesoria, o bien aportar una información con valor generalizador. En la caracterización de este autor entran en juego los conceptos de tema y rema, relacionados cada uno de ellos con un tipo de relativa y con un tipo de proyección, entendida esta en términos anafóricos y catafóricos:

Inspirándonos en las teorías de Firbas (1971) sobre el dinamismo comunicativo, el tema (o tópico) se asociaría al elemento menos portador de información -es decir, a las estructuras de tipo explicativo-, mientras que el rema (o comento) se vincularía al elemento que hace avanzar el texto -las estructuras especificativas-[...]. El proceso explicativo en las relativas resultaría naturalmente anafórico o retrospectivo, puesto que redundaría en los aspectos de significado más prescindibles de un ente $\mathrm{o}$, gramaticalmente, de un antecedente. Al contrario, el proceso especificativo permitiría el avance denotativo, ya que aportaría aspectos particularizadores e indispensables sobre el elemento al que completa la estructura relativa; en tal caso, se percibiría naturalmente catafórico o prospectivo (Rodríguez, 2013: 146-147).

Así pues, los elementos relativos que servirán de base para la búsqueda en el corpus de trabajo ( todos ellos con unas implicaciones en el discurso informativo) son los siguientes: 
Tabla 1. Implicaciones de la elaboración hipotáctica en el discurso informativo

\begin{tabular}{|c|c|}
\hline Elementos de búsqueda & Estilo comunicativo \\
\hline $\begin{array}{c}\text { Pronombres relativos } \\
\text { (que, cual, quien y variantes) }\end{array}$ & \multirow{3}{*}{$\begin{array}{l}\text { El uso de oraciones explicativas en el } \\
\text { discurso informativo se corresponde con la } \\
\text { progresión informativa, el dinamismo discursivo. } \\
\text { Las oraciones explicativas contribuyen a la } \\
\text { profundización de la información y al estilo } \\
\text { cohesionado. } \\
\text { El uso de un tipo u otro de relativo está } \\
\text { relacionado con el grado de formalidad del texto } \\
\text { y con la temática de la información. }\end{array}$} \\
\hline $\begin{array}{c}\text { Determinantes } \\
\text { relativos (cuyo y variantes) }\end{array}$ & \\
\hline $\begin{array}{l}\text { Adverbios relativos } \\
\text { (donde, como, cuando y } \\
\text { variantes) }\end{array}$ & \\
\hline
\end{tabular}

IV. Metodología: análisis de un corpus con anotaciones sintácticas

Para realizar el análisis, se ha elaborado un corpus de noticias periodísticas de autor - firmadas por periodistas de la casa y no de agencias-, recopilado durante los meses de febrero y marzo de 2014 y extraído de seis periódicos: dos de tipo generalista, dos económicos y dos deportivos, seleccionados principalmente por variedad editorial. Como cada corpus consta de un número de palabras distinto, se ha optado por normalizar las frecuencias absolutas por cada 100000 palabras de texto. En el cómputo de palabras de cada corpus se incluyen todos los elementos de la noticia (titular, entradilla y cuerpo de la noticia), excepto los datos referentes a autoría, sección, ciudad o fecha de publicación.

Tabla 2. Caracterización del corpus

\begin{tabular}{|c|c|c|}
\hline Periódico & № de noticias & № de palabras \\
\hline El País & 1360 & 903848 \\
\hline La Razón & 1076 & 597137 \\
\hline Generalistas & 2436 & 1501807 \\
\hline Expansión & 905 & 419986 \\
\hline Cinco Días & 549 & 314547 \\
\hline Económicos & 1454 & 734533 \\
\hline As & 1025 & 308521 \\
\hline Sport & 991 & 415590 \\
\hline Deportivos & 2016 & 724111 \\
\hline
\end{tabular}

Para realizar el análisis, llevado a cabo con la herramienta Concordance del programa WordSmith Tools, en su versión 7.0. (Michael Scott, 2018), el corpus se ha etiquetado mediante el sistema VISL, Visual Interactive Syntax Learning, un proyecto de investigación y desarrollo del Institute of Language and Communication, de la University of Southern Denmark (SDU), dirigido por el lingüista Eckhard Bick. Concretamente, la herramienta utilizada es Hispal Parser (Constraint Grammar-Based Parser for Spanish), un etiquetador con anotaciones morfológicas y sintácticas basado, en parte, en un proyecto anterior 
en lengua portuguesa: Palavras. Según las pruebas e investigaciones de Bick (2006), aunque no hay datos que lo puedan comparar con otros etiquetadores en español, como Connexor's Machinese (http://www.connexor.com/demo/syntax/) o Freeling (Padró et al.), la precisión sintáctica de Hispal Parser es de un 95-96 \%. Además de esta cifra, varias razones de peso han influido para utilizar este programa para nuestro corpus de estudio:

a. Por un lado, su accesibilidad. Aunque el programa es de pago (como la mayoría), dispone de una versión de uso libre en su página web (https://visl.sdu.dk/visl/es/parsing/automatic/upload.ph p), en la que cualquier usuario puede subir sus textos originales, aunque limitados a un tamaño concreto. El programa ofrece un enlace con el texto ya etiquetado pasados unos minutos (el tiempo de espera depende del tamaño del texto original). Una de las condiciones para utilizar esta interfaz es que los textos subidos por el usuario pasarán a formar parte de la base de datos del proyecto.

b. Por otro lado, su usabilidad, entendida como efectividad y practicidad. El usuario no precisa tener conocimientos informáticos avanzados, ya que la versión libre no requiere de ninguna instalación ni mediante la línea de comandos ni a través de instaladores.

c. Por último, su sistema de anotación. Hay varios etiquetadores morfológicos en español; sin embargo, ninguno de ellos contaba con una anotación tan concreta como Hispal Parser. Para nuestro análisis, necesitábamos discernir, por ejemplo, el que conjunción del que pronombre $y$, tras varias pruebas con otros etiquetadores, ninguno ofrecía la precisión de este.

d. ¿Por qué utilizar un corpus anotado? Aunque desde el primer momento del estudio teníamos en mente las palabras del creador de WordSmith Tools, Michael Scott (en comunicación personal), de que cuanto más limpio esté un corpus, más objetivos son los resultados obtenidos, también queríamos que las búsquedas fueran prácticas. En este sentido, no era lo mismo buscar cada una de las formas de los relativos (con todas sus variantes con y sin preposición, y con y sin artículo) -con la consiguiente limpieza manual para distinguir, por ejemplo, el que conjunción del que relativo-, que buscar directamente la etiqueta de relativo $(\langle r e \mid\rangle)$ en el corpus anotado. Con todo, cabe decir que siempre hay que 
revisar las concordancias obtenidas para eliminar

aquellas búsquedas que no concuerdan con los objetivos.

Los elementos que han sido clave para buscar pronombres, determinantes y adverbios relativos en oraciones explicativas han sido los signos de puntuación, por ser esta la norma general. De esta forma, se han tenido en cuenta los contextos en los que aparecían comas, paréntesis y rayas $^{6}$, con objeto de excluir las oraciones especificativas de las explicativas desde el primer momento de la búsqueda. Por tanto, se han obviado las posibles excepciones de explicativas sin pausa; sin embargo, sí se han eliminado las especificativas con pausa introducidas por el que, la que, los que y las que, al tiempo que se han obviado los resultados con encapsuladores anafóricos. Con las concordancias obtenidas, se ha realizado una revisión posterior para discriminar aquellos elementos que no cumplían los requisitos de las explicativas, a saber: el adverbio cuando sin antecedente temporal o con valor concesivo o condicional; oraciones con como con valor causal, condicional, preposicional $^{7}$ o ejemplificativo, o las oraciones con que, precedidas de conjunción, como y o pero, que no siempre son relativas explicativas $^{8}$ :

8. Sin antecedente: En la selección brasileña, cuando Neymar recibe una falta ostentosa, Marcelo es el más puntual en acudir al rescate, ponerse de uñas con el agresor, y reparar en el árbitro. (Dídac Peyret, «Enemigos íntimos», Sport, 20-3-2014, 8).

9. Valor condicional: Es blanca como la nieve, porque está esculpida directamente en la roca, y ese material, cuando se trabaja, parece escayola o talco. (Ulises Fuente, "Egipto confidencial», La Razón, 19-3-2014, 56).

10. Valor preposicional: La Fiscalía de la Audiencia Nacional solicitó al juez Pablo Ruz la imputación del Barcelona (como persona jurídica) en un delito fiscal en el fichaje de Neymar, al considerar que el club defraudó 9,1 millones a Hacienda. (Moisés Llorens, "La Fiscalía pide imputar al Barça por Neymar», As, 20-2-2014, 2).

11. Valor causal: En consecuencia, como el niño no está acostumbrado a que le fijen normas, se rebela y tiene más riesgo de querer fugarse. (Raquel Vidales, «Coger la puerta... y volver otra vez», El País, 18-3-2014, 30).

\footnotetext{
${ }^{6}$ También se ha considerado buscar el guion corto, puesto que en algunos medios se utiliza este para delimitar los incisos.

7 Jiménez (2003) habla del valor de como como preposición en casos donde no aparece el verbo. Sin embargo, para otros autores, se trata de un valor relativo donde hay un verbo omitido.

${ }^{8}$ De hecho, se ha comprobado que, en algunos de estos casos, el programa ha etiquetado incorrectamente la conjunción que, introductoria de una subordinada sustantiva, como un relativo.
} 
12. Conjunción: La realidad fue que el Bayern se gustó, nos gustó, y

que hoy es claro favorito para pasar a cuartos. (Edu Fernández, «El Arsenal se agarra al pasado para hacer posible el milagro», As, 113-2014, 11).

13. Conjunción: Agregaba el oficial que su organización estaba fuera de la política, pero que, a partir de los sangrientos sucesos de los últimos días, su grupo y otros grupos de defensa forman parte del consejo coordinador del Maidán que hasta ahora había estado controlado por los líderes de la oposición parlamentaria. (Pilar Bonet, «El Maidán recela de los políticos», El País, 22-2-2014, 4).

\section{Resultados: relativas explicativas en el mensaje periodístico}

Numerosos autores que estudian el estilo periodístico hacen referencia a la subordinación en el discurso informativo y, más concretamente, a las funciones de las oraciones de relativo explicativas en este ámbito de uso. Vellón (2013: 92) destaca que la incidencia del relativo en el discurso informativo es el síntoma de una paradoja característica de este lenguaje: por un lado, contribuye a ofrecer el conjunto completo de datos de una información y, por otro lado, fomenta el estilo cohesionado frente al segmentado, que es más dinámico y el recomendado por los libros de estilo.

La ampliación de la información aportada por las oraciones explicativas también tiene que ver, según Vellón (2013: 92), con las carencias del estilo nominal, puesto que las formas nominalizadoras del discurso no son suficientes para la especificación informativa que requiere el texto noticioso.

Pero ¿hay diferencias en los resultados obtenidos según el modelo de cada medio? La siguiente tabla muestra los datos absolutos y normalizados (por cada 100000 palabras) de las oraciones explicativas, recogidas en cada una de las cabeceras de análisis, y según el relativo introductorio de la construcción: 
Tabla 3. Elaboración hipotáctica. Generalistas y especializados

\begin{tabular}{|c|c|c|c|c|c|c|c|}
\hline \multicolumn{2}{|l|}{ Tipo de medio } & \multicolumn{2}{|c|}{ GENERALISTAS } & \multicolumn{2}{|c|}{ DEPORTIVOS } & \multicolumn{2}{|c|}{ ECONÓMICOS } \\
\hline \multicolumn{2}{|c|}{ Relativos $\quad$ No de palabras } & 903848 & 597137 & 308521 & 415590 & 419986 & 314547 \\
\hline \multirow{2}{*}{$\begin{array}{c}\text { que + variantes/ } \\
\text { el que+ variantes }\end{array}$} & FA & 3055 & 2045 & 1010 & 1125 & 1848 & 1269 \\
\hline & FN & 338 & 342 & 327 & 271 & 440 & 403 \\
\hline \multirow[t]{2}{*}{ quien + variantes } & $\mathrm{FA}$ & 202 & 220 & 57 & 176 & 50 & 37 \\
\hline & FN & 22 & 37 & 18 & 42 & 12 & 12 \\
\hline \multirow{2}{*}{$\begin{array}{r}\text { el cual + } \\
\text { variantes }\end{array}$} & FA & 49 & 42 & 11 & 12 & 15 & 17 \\
\hline & FN & 5 & 7 & 4 & 3 & 4 & 5 \\
\hline \multirow[t]{2}{*}{ cuyo + variantes } & $\mathrm{FA}$ & 160 & 101 & 30 & 38 & 83 & 57 \\
\hline & $\mathrm{FN}$ & 18 & 17 & 10 & 9 & 20 & 18 \\
\hline \multirow[t]{2}{*}{ donde+ variantes } & FA & 448 & 278 & 112 & 168 & 150 & 126 \\
\hline & FN & 50 & 47 & 36 & 40 & 36 & 40 \\
\hline \multirow[t]{2}{*}{ cuando } & $\mathrm{FA}$ & 270 & 157 & 72 & 91 & 89 & 69 \\
\hline & FN & 30 & 26 & 23 & 22 & 21 & 22 \\
\hline \multirow[t]{2}{*}{ como } & FA & 305 & 273 & 100 & 146 & 123 & 79 \\
\hline & FN & 34 & 46 & 32 & 35 & 29 & 25 \\
\hline \multirow[t]{2}{*}{ TOTAL } & FA & 4489 & 3116 & 1392 & 1756 & 2358 & 1654 \\
\hline & FN & 497 & 522 & 451 & 423 & 561 & 526 \\
\hline
\end{tabular}

En primer lugar, respecto a los resultados obtenidos en el uso de oraciones explicativas introducidas por el pronombre que, hay que destacar que se ha registrado el mayor número de casos en comparación con los demás relativos, como cabría esperar por ser el relativo de uso más general en español. La ausencia de rasgos léxicos y morfológicos de este relativo permite que aparezca en un gran número de contextos (NGLE, 2009: § 22.4h).

Así, en cuanto a que, los relativos complejos - el que, la que, lo que, los que, las que- y sus variantes con preposición, los periódicos económicos han obtenido las frecuencias más altas: Expansión registra 440 oraciones explicativas introducidas por que o alguna de sus variantes por cada 100000 palabras, y Cinco Días obtiene 403 casos. A los periódicos económicos les siguen los generalistas ( $L a$ Razón: 342/100 000; El País: 338/100 000 palabras). Los deportivos son los que menos explicativas de este tipo registran, con 327 casos de As y 271 de Sport. Lo que llama la atención de estos datos es que en los periódicos económicos se ha recogido el mayor número de casos del relativo complejo con pronombre neutro lo que, que presenta antecedentes oracionales ${ }^{9}$ cuyo contenido -global 0 parcial - es recogido en forma pronominal por el relativo:

\footnotetext{
${ }^{9}$ Cabe señalar que también pueden introducir relativas explicativas con antecedente oracional los pronombres lo cual y que y el adverbio como, según la NGLE (2009: 44.8I).
} 
14. Según la CNMC, desde julio de 2009, en que se crearon las subastas eléctricas, hasta octubre de 2013 , el coste ha sido un $11 \%$ superior a los precios de mercado (1.670 millones), lo que se tradujo en un sobrecoste en la factura del 4,3\%. (Carmen Monforte, "La CNMC pide la desaparición de la tarifa eléctrica para los domésticos», Cinco Días, 01-3-2014, 3).

15. El precio objetivo a 12 meses del conjunto de los expertos para el valor es de 30,88 euros, lo que implica un potencial de revalorización del 5,5\%. (Miriam Calavia, "Valores para sacar partido de la calma tensa del mercado», Cinco Días, 20-3-2014, 20).

16. Éste incluye más ayudas públicas de las inicialmente previstas y una reclasificación de otras anteriores ya aprobadas, lo que obligará a Ceiss a realizar mayores ajustes. (M. Roig, M. Martínez, «Bruselas da luz verde a la compra de Ceiss por Unicaja», Expansión, 13-3-2014, 14).

17. Entre los argumentos que apoyan esta hipótesis está que el invierno de este año ha sido particularmente suave, lo que ha permitido acumular un stock para los próximos 4 ó 5 meses. (Pablo J. Cerezal, «Europa impulsa el gas por 'fracking' para blindar el suministro ante crisis rusas», Expansión, 19-3-2014, 24).

Algunos autores, como García (1996) o Martínez (1994), llaman a este tipo de oraciones "construcciones continuativas», puesto que a veces adquieren un valor ilativo, similar a locuciones como por 10 tanto, pues o por consiguiente. La reiterada aparición de este relativo complejo en los periódicos especializados en economía evidencia que esta temática requiere de explicaciones complejas de causaefecto. En esta misma línea se sitúa la combinación del pronombre indefinido neutro algo con el relativo que, que actúa de forma similar al relativo complejo lo que y que se ha registrado en ejemplos como este:

18. Ahora, está pendiente de ver si se hace con un contrato en Talara, (Perú), lo que aportaría en torno a 1.050 millones de euros a sus cuentas, algo que aplaudirá el mercado, según Renta 4. (D. Esperanza, "Técnicas Reunidas, una oportunidad a medio plazo", Expansión, 17-3-2014, 20).

Con respecto a los resultados obtenidos en el uso del pronombre quien, el dato más reseñable se ha obtenido en los periódicos especializados económicos, con cifras bastante inferiores a las de la prensa generalista y deportiva. Se han registrado las siguientes frecuencias, por orden: As (18), El País (22), La Razón (37) y Sport (42). Por su parte, las dos cabeceras económicas usan 12 oraciones de este tipo por cada 100000 palabras. El presente dato demuestra que este tipo de medio informa poco de personajes o entes personificados; más bien se nutre y comunica a partir de informes, datos, organizaciones, empresas, instituciones y demás entidades. En 
cambio, tanto la prensa generalista como la deportiva utilizan numerosas fuentes personales, sobre todo en una tipología de noticias, las informaciones declarativas, que convierten en texto noticiable las palabras de las figuras públicas, y en las que la relativa introducida por quien está al servicio del discurso reportado. En ambas cabeceras generalistas, su uso destaca especialmente en la sección de política, en la que se han obtenido más concordancias (54 casos registrados en La Razón; 28 casos en El País). De hecho, las secciones de política y deportes son las más asiduas a esta práctica porque muchas de sus informaciones proceden de comunicados, comparecencias o ruedas de prensa. Cabe matizar, además, que estas cláusulas explicativas introducidas por quien son fundamentales para aportar una información remática sobre el antecedente humano, ya que inician un nuevo recorrido informativo a partir del pronombre, que actúa como enlace temático, hasta el punto de que en muchas ocasiones focaliza el interés informativo:

19. "Queremos construir, estamos dispuestos a aportar, pero cuando se está en el Gobierno es muy importante asumir la responsabilidad". "Creo que estamos en un gran partido", enfatizó el líder de los populares, quien trató de resumir su hoja de ruta política con meridiana claridad: "ETA, a disolverse, no hay referéndum que liquide la soberanía nacional y España tendrá pronto crecimiento económico y empleo". (J. Rivas, "Rajoy emplaza a ETA ante el PP vasco: "Se arregla muy fácil: disuélvanse"», El País, 09-3-2014, 31).

20. Ningún socialista se paró ante los medios, salvo la secretaria de Política Municipal de la Ejecutiva del PSN, Maite Esporrín, quien a la salida de la reunión extraordinaria confirmaba la decisión de su grupo de apoyar una moción de censura. (Macías Alfonso Merlos, "La solución imposible del PSN: echar a Barcina sin el apoyo de Bildu», La Razón, 02-3-2014, 22).

Una función similar adquiere el adverbio relativo como cuando se usa entre incisos. La única diferencia entre ambos es su antecedente: quien tiene como antecedente a la fuente, mientras que como tiene como antecedente las palabras de la fuente, por lo que se trata de un antecedente oracional. Independientemente de su debatida identificación como oración de relativo explicativa o como relativa libre -entre todos los valores que ofrece esta polifacética unidad gramatical-, su uso como relativo en la prensa periodística es superior al de otras partículas relativas. Destaca, especialmente, en los modelos generalistas y deportivos (la cifra oscila en una FN de 32 a 46 casos) y, como ocurría con quien, tiene un uso más limitado en las cabeceras económicas (Expansión: 29/100 000; Cinco Días: 25/100 000). Uno de sus principales 
cometidos es, pues, recuperar o dar paso $^{10}$ a las palabras de las fuentes informativas, o incluso rescatar contenidos ya dados por el periódico días anteriores, de modo que con la autorreferencialidad se aporta credibilidad a la información:

21. Ahora la precaución llega del Este de Europa, aunque sorprendentemente con algún efecto colateral positivo, como señala Luis Antonio Ruiz, presidente de Jaguar-Land Rover España: "Nos van a pasar producción prevista para Ucrania, porque nos faltaban coches para cubrir la demanda de aqui". (M. Gómez Blanco, «El final del túnel está en el Sur», El País, 8-3-2014, 55).

22. Con más o menos tiempo, el cantante vitoriano llega como el veterano de la gala después de quedar segundo en su certamen homólogo del año 2000. Ahora, para intentar su asalto a Dinamarca, Raúl se presenta con "Seguir sin ti», una balada "sobria, elegante y madura», como él mismo la calificó. (G. Granda, J. Herrero, "El camino a Eurovisión se despeja esta noche», La Razón, 22-2-2014, 92).

23. En los últimos meses habían aparecido rumores en todas las direcciones posibles sobre el futuro de Ter Stegen pero, finalmente, el portero alemán jugará la próxima temporada en el Camp Nou, como apuntaba uno de ellos. (David Salinas, "Ter Stegen superó la revisión médica», Sport, 27-2-2014, 4).

24. Como ya adelantó AS, la coincidencia de fechas del anuncio oficial del fichaje del madrileño por Peugeot para correr la próxima edición del Dakar ha sido un impedimento insalvable para su participación en Canarias. (Pipo López, «Sainz no correrá al final en Canarias», As, 19-3-2014, 38).

25. Parte de la banca acreedora ha capitalizado deuda y se prevé que ahora traspase su participación a una gestora de activos tóxicos, como adelantó EXPANSIÓN. (Marisa Anglés, "Colonial elevará el importe de la ampliación», Expansión, 1-3-2014, 10).

Ahora bien, hay que destacar que, en otras ocasiones, tanto quien como como encabezan relativas que concretan acciones del personaje en cuestión:

26. La petición de 30 días de suspensión parte del magistrado instructor, a quien el Consejo del Poder Judicial encomendó investigar si Vázquez Taín cometió una falta muy grave por las

\footnotetext{
${ }^{10}$ Como da cuenta la NGLE (2009: § 22.11f), estos incisos con como pueden aparecer a principio de oración, intercalados o al final, de forma que la relativa puede aparecer delante de su antecedente. Por esta razón, para el análisis de este relativo, también se han tenido en cuenta las relativas que aparecen a principio de oración, precedidas de punto.
} 
supuestas irregularidades cometidas en la recusación que le interpuso el principal implicado en el robo del Códice. (José A. Hernández, «El instructor del Consejo pide suspender un mes al juez Vázquez Taín», El País, 12-3-2014, 13).

27. El doctor Berger, quien le permitió "volver a jugar al tenis" en 2010, le recomendó parar y evitar males. (Neus Yerro, "Del Potro se enfrenta a un futuro incierto». Sport, 11-3-2014, 39).

28. En esta reunión el banquero no pudo ocultar su emoción, e incluso, como le ocurrió un día antes, se le escapó alguna que otra lágrima. (Ángeles Gonzalo, "El banquero sin activos tóxicos», Cinco Días, 01-3-2014, 40).

Con todo, cabe mencionar que también el relativo que puede tener como antecedente nombres propios. En la mayoría de casos obtenidos, adquiere esta última función de quien: se usa más para precisar acciones, estados o cualidades del antecedente que para introducir el discurso reportado:

29. La primera, a la Mejor Película de Animación y Mejor Canción interpretada por Idina Menzel (que actuó en la gala). (Rosa Gamazo, David O. Russell, «Blanchett y McConaughey, los mejores actores de esta edición», La Razón, 04-3-2014, 50).

30. Según Tania Akouche, que se muestra sorprendida ante la detención de su padre en España, Hatem regresó a Valencia en enero pasado para someterse a una revisión junto a su hijo donante, Ali Hatem. (Laura J. Varo, "Dijeron que el órgano de mi hermano no servía"», El País, 13-3-2014, 36).

En referencia a los relativos el cual y cuyo (y variantes), las frecuencias obtenidas revelan que se usan poco en el lenguaje periodístico, algo que también se refleja en otros ámbitos, especialmente en los registros informales, desde hace décadas. Álvarez (1988: 91) señala que «ninguno de los dos puede, pues, considerarse al mismo nivel que los otros relativos en cuanto al uso, y ninguno de ellos mantiene tampoco, en la sincronía actual, la vitalidad que poseyeron en tiempos pasados». Por una parte, las frecuencias normalizadas obtenidas de el cual y sus variantes oscilan entre los 3 casos por cada 100000 palabras (Sport) y los 7/100 000 (La Razón). Por otra parte, las frecuencias del determinante relativo posesivo cuyo, relativamente superiores al anterior, oscilan entre los 9 y los 20 casos. Destacan especialmente las bajas cifras en los periódicos deportivos ( 9 casos en Sport y 10 en As), lo que evidencia que el grado de formalidad de estos textos está más cercano al lenguaje coloquial: 
31. Se da por hecho que los miembros del consejo den luz verde al pacto, cuyos detalles se han estado perfilando desde noviembre. (Miguel Ángel Patiño, «Repsol celebra hoy dos consejos: por YPF y por las cuentas», Expansión, 25-2-2014, 4).

32. Mañana viernes se celebrará el primer interrogatorio ante el juez central de Instrucción 1, Javier Gómez Bermúdez, que investiga la implicación de ambos en el envío de un paquete desde Toledo a Madrid en 1991, a nombre de Jesús Gallego, de Construcciones Atocha, una de las adjudicatarias de la autovía de Leizarán, a cuyo trazado se oponía ETA. (María Fabra, "Prisión para los etarras detenidos en México», El País, 20-2-2014, 19).

Los adverbios relativos de lugar y tiempo, donde y cuando, manifiestan una de las particularidades de la noticia como género: su carácter narrativo. A este respecto, consideramos que la noticia presenta una estructura plurisecuencial con un patrón discursivo predominante: el expositivo-narrativo (Vellón, 2014). La aparición de estos relativos evidencia las marcas de narratividad de la noticia porque supone contextualizar lo sucedido, los acontecimientos. Donde admite antecedentes espaciales como lugares, pero también acciones, eventos o situaciones que incluyen de manera implícita coordenadas espacio-temporales (NGLE, 2009: $\S 22.81$ ). Cuando, el conector temporal más empleado en el español actual, según recoge la NGLE, sitúa un evento o designa instantes o momentos. En el análisis del corpus, tanto uno como otro resaltan en un modelo de medio: el generalista. Donde y sus variantes aparecen 50 veces por cada 100000 palabras en El País, cifra similar a la registrada en La Razón (47), mientras que en los periódicos especializados - tanto económicos como deportivos - la cifra oscila entre las 36 y 40 concordancias. Cuando se registra en El País con una FN de 30 casos por cada 100000 palabras, mientras que en La Razón la cifra es de 26. Los especializados oscilan entre los 21 y los 23 casos. El uso de estos adverbios en relativas explicativas, que añaden alguna aclaración a la contextualización espacio-temporal de los hechos relatados en la noticia o de sus antecedentes y consecuencias, muestra que el estilo de los periódicos de información general (con temáticas variadas, de carácter social, cultural, deportivo, económico y político) se sirve de este recurso para profundizar en las circunstancias que rodean el que informativo, como una estrategia de diferenciación frente a la inmediatez de su gran competencia, la prensa digital:

33. En 1974, cuando los anticonceptivos estaban prohibidos en España, la Fiscalía del Tribunal Supremo estimó que pese a que se consideraba un delito en cualquier circunstancia, en España se 
realizaban unos 300.000 abortos al año -cifra que los más

conservadores consideraron disparatada-. (María Sahuquillo, «Tantas razones como mujeres», El País, 12-3-2014, 35).

34. Es el caso de Bielorrusia, donde la UE - más proclive a negociar que a sancionar - ha vetado el visado para viajar a países miembros de la Unión a 232 ciudadanos y ha congelado las cuentas de 25 empresas. (Belén Domínguez, «El peligroso efecto de las sanciones», El País, 10-3-2014, 30).

\section{Conclusiones}

Después de analizar, desde el punto de vista cuantitativo, las oraciones de relativo explicativas en los tres modelos de periódicos, podemos establecer una caracterización de los medios generalistas y especializados a partir del uso y las funciones de las relativas explicativas.

Si comparamos los datos generales de cada una de las cabeceras, nos encontramos con un predominio de las construcciones explicativas en los medios económicos: Expansión destaca con 561 casos, mientras que Cinco Días usa 526 oraciones de este tipo por cada 100000 palabras. Queda claro que se trata de medios que abogan por el estilo cohesionado, en el que resalta la explicación, aclaración o especificación del hecho noticioso en un sentido: acentúan más las causas y las consecuencias que la contextualización espaciotemporal o concerniente a antecedentes personales. Esto demuestra que sus fuentes y actores principales no son personas individuales, sino organismos, empresas y entidades diversas. Además, el hecho de usar relativos como el cual o cuyo evidencia que se trata de medios con un grado de formalidad medioalto.

En el lado opuesto se sitúan los medios especializados en deportes. As usa 451 relativas explicativas, mientras que Sport tiene una frecuencia normalizada de 423 casos. Estas cifras señalan el predominio de un estilo más dinámico en este tipo de medios, que usan un estilo segmentado cercano a la oralidad. Además, su escasa preferencia por relativos cultos como cuyo también pone de manifiesto que el nivel de formalidad de estos textos es más bajo que en los otros modelos. Por otro lado, resalta la predilección por los actores y fuentes personales, algo que se relaciona con el estilo declarativo de sus noticias.

Con respecto a los medios generalistas, estos se sitúan en un estilo intermedio entre los medios especializados económicos y los deportivos. Respecto a las cifras totales, destaca La Razón con 522 casos, mientras que El País usa 497 oraciones explicativas. Si destacan en algún aspecto con respecto a los otros modelos, es en el 
uso de relativas explicativas con antecedentes circunstanciales, lo que se traduce en que la profundización en la contextualización de los hechos es un plus para la prensa escrita de información general, que tiene que diferenciarse de la prensa en Internet y su actualización constante. Asimismo, el uso de relativos con antecedentes personales desempeña el mismo papel que en la prensa deportiva especializada: favorece el estilo declarativo.

Sobre la comparativa entre los medios generalistas, no podemos extraer datos concluyentes respecto al lenguaje de cada tipo de sección con únicamente dos cabeceras. Se debería ampliar el estudio de otras publicaciones de información general para comprobar si se vislumbra un patrón en el estilo de las distintas secciones. Finalmente, en este estudio hemos obviado las relativas externas de participio y gerundio (que algunos autores enmarcan dentro de las oraciones de relativo). Se trata de construcciones que modifican a un sustantivo antecedente y que funcionan a modo de inciso, por lo que podría ser interesante considerarlas en próximas investigaciones.

\section{Bibliografía}

Álvarez Martínez, Ma Ángeles. 1988. "Los pronombres "el cual" y "cuyo". ¿dos relativos en desuso?». Revista de Filología. Universidad de La Laguna 6 y 7: 79-92.

Bick, Eckhard. 2006. "A Constraint Grammar Parser for Spanish». Proceedings of TIL 2006/4th Workshop on Information and Human Language Technology/ (Ribeirão Preto, octubre, 27-28).

Borrat, Héctor. 1993. "Hacia una teoría de la especialización periodística». Análisi. Quaderns de Comunicació i Cultura 15: 7984.

Borreguero Zuloaga, Margarita. 2018. "Los encapsuladores anafóricos: una propuesta de clasificación». Caplletra. Revista Internacional de Filologia 64: 179-203.

Casero-Ripollés, Andreu, ed. 2004. Periodismo político en España: concepciones, tensiones y elecciones. La Laguna: Sociedad Latina de Comunicación Social.

Chillón, Albert. 2001. "El 'giro lingüístico' en periodismo y su incidencia en la comunicación periodística». Cuadernos de Información 14: 24-47.

Company Rico, Juan Antonio. 2015. Las construcciones relativas y sus distintos ámbitos: un estudio contrastivo francés/español. València: Universitat de València.

Del Río, Rosa. 2010. «Noticias económicas, el poder de la información», en La especialización en el periodismo: Formarse 
para informar, coordinado por Idoia Camacho, 100-126. SevillaZamora: Comunicación Social.

Downing, Angela y Philip Locke. 1992. A University Course in English Grammar. Londres: Prentice-Hall.

García García, Serafina. 1993. "Las fronteras de los pronombres relativos». Verba: Anuario Galego de Filoloxia 20: 355-368.

Gómez Bueno, Javier. 2014. «La mezcla de géneros periodísticos en la prensa deportiva escrita en Cataluña». Razón y palabra 87. Acceso en

http://www.razonypalabra.org.mx/N/N87/N87/06_Gomez_V87. pdf.

González Rodríguez, Mạ José. 2003. «Relaciones lógico-semánticas y su forma de expresión en el género noticia». Atlantis 25 (2): 5559.

Gutiérrez Araus, Mạ Luz. 2004. Problemas fundamentales de la gramática del español como 2/L. Madrid: Arco Libros.

Halliday, Michael A. K. 1985. An Introduction to Functional Grammar. Londres: Edward Arnold.

Halliday y Ruqaiya Hasan. 1976. Cohesion in English. Nueva York: Longman.

Halliday, Michael, Christian Matthiessen. 2014. Halliday's Introduction to Functional Grammar. Londres: Routledge.

Jiménez Juliá, Tomás. 2003. “Como” en español actual». Verba: Anuario Galego de Filoloxia 30: 117-161.

López Samaniego, Anna. 2013. "Las etiquetas discursivas: del mantenimiento a la construcción del referente». Estudios de Lingüística Universidad de Alicante 27: 167-197.

Martínez Lanzán, Gloria. 2010. «Los extranjerismos en la prensa económica». En Los caminos de la lengua. Estudios en homenaje a Enrique Alcaraz Varó, editado por José Luis Cifuentes et al, 709-730. Alicante: Publicaciones Universidad de Alicante.

Martínez, José Antonio. 1994. Funciones, categorías y transposición. Madrid: Istmo.

Moya Isach, Rosana. 2018. «Conexión textual y modelos comunicativos: la elaboración paratáctica en el discurso periodístico informativo». Cultura, lenguaje y representación 9: 99-118

Naranjo de Arcos, Alicia. 2011. Tratamiento de la información deportiva en la prensa: la crónica como género prevalente. El caso de los encuentros de fútbol entre Real Madrid y F.C. Barcelona. Tesis doctoral. Málaga: Universidad de Málaga. 
Páez Cordero, Florelia. 2013. El uso de los pronombres relativos en el español de Venezuela. Oviedo: Universidad de Oviedo.

Pano Alamán, Ana. 2012. "Aproximación al discurso sobre la prima de riesgo en los titulares de economía de la prensa española». Discurso \& Sociedad 6-4: 759-781.

Real Academia Española y Asociación de Academias de La Lengua Española. 2009. Nueva gramática de la lengua española, volúmenes I (Morfología y Sintaxis) y II (Sintaxis). Madrid: Espasa Libros.

Rodríguez Muñoz, Francisco José. 2013. «La proyección sintagmática de las oraciones relativas en español». Onomázein: Revista de lingüística, filología y traducción 28: 143-147.

Van Dijk, Teun A. 1990. La noticia como discurso: comprensión, estructura y producción de la información. Barcelona: Paidós.

Vellón Lahoz, Francisco Javier. 2013. Norma y estilo en los libros de redacción. Salamanca: Comunicación Social.

Vellón Lahoz, Francisco Javier. 2014. «Divulgación y didacticidad en el discurso periodístico: la información económica durante la crisis». Zer. Revista de Estudios de Comunicación 19: 99-119. 


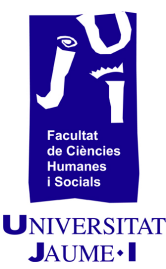

\section{La enseñanza de las locuciones como recurso lingüístico en los textos de Bachillerato}


I. Resumen

\begin{abstract}
l. Resumen
El objetivo de este trabajo es analizar la presencia de las locuciones en los manuales de Lengua Castellana y Literatura de 1은 de Bachillerato, así como conocer qué tipo de actividades se proponen en ellos. En primer lugar, se explica la metodología y la descripción del corpus, el cual posibilita la observación y la extracción de los resultados obtenidos de forma objetiva. En segundo lugar, se exponen las características formales más relevantes de las locuciones y la clasificación establecida en el Manual de la Nueva gramática de la lengua española (2010). Seguidamente, se compara su enseñanza en la Educación Secundaria Obligatoria con la del aula de Español como Lengua Extranjera, dado que esta última sitúa las locuciones en una posición más relevante dentro del currículo.
\end{abstract}

Palabras clave: educación, aprendizaje, lengua española, fraseología, locuciones, recurso lingüístico.

\title{
II. Introducción
}

El objeto de estudio de este trabajo es el tratamiento de las locuciones en los manuales de Lengua Castellana y Literatura de 10 de Bachillerato, en los que la enseñanza de este tipo de expresiones fijas apenas tiene cabida. Además, en los niveles superiores, como es este caso, todavía son, como señala Martínez Marín (2000, 328), «insuficientemente atendidas en la enseñanza del castellano, aunque tienen una gran importancia en su estructura y funcionamiento».

Por el contrario, en la enseñanza de español como lengua extranjera, tanto las locuciones como otras unidades pertenecientes a la fraseología son una parte fundamental del currículo, porque sin ellas los alumnos no entenderían muchas de las expresiones que se utilizan con frecuencia en las conversaciones. Asimismo, cada vez más manuales que integran y trabajan las locuciones. Por ejemplo, se han publicado obras como Locuciones y refranes para dar y tomar. El libro para aprender más de 120 locuciones y refranes del español, en el que se destaca la importancia de «trabajar las locuciones de manera integrada en el resto de contenidos del currículo, de modo que no queden aisladas y descontextualizadas» (Gómez González y Ureña Tormo 2015, 3).

Sin embargo, sea como fuere, la poca presencia que adquieren las locuciones en el aula de Secundaria conlleva un empobrecimiento en la competencia comunicativa y cultural de los jóvenes, quienes se ven obligados, en muchos casos, a emplear proformas o palabras baúl en vez de otros elementos lingüísticos como las locuciones, que 
les permitirían dar un notable salto cualitativo y enriquecer con numerosos matices el mensaje (Núñez-Román 2015, 156). Estas expresiones tienen, de algún modo, una belleza especial que hace que recurra a ellas cuando se quiere decir más de lo que se está diciendo, dado que, evidentemente, no se expresa con la misma fuerza ilocutiva llueve mucho que llueve a cántaros. Es por eso por lo que algunos investigadores «sugieren que quizá es la gramática tal y como se enseña lo que no ayuda a los alumnos a mejorar los usos» (Fontich 2014, 5). En definitiva, se trata de dejar constancia de la poca claridad a la hora de trabajar este aspecto lingüístico que, en muchos casos, queda casi invisibilizado en el aula.

\section{Objetivos}

El objetivo principal es conocer cuál es el lugar que ocupan las locuciones en los manuales de texto y, así, entender cómo se lleva a la práctica su enseñanza en el aula. Por eso, consideramos importante analizar y comparar el tratamiento de las locuciones en los manuales de Lengua Castellana y Literatura de 10 de Bachillerato a través de un cuestionario general para identificar qué clases de locuciones se explican y si se contextualizan dentro de un texto.

\section{Las locuciones como elemento didáctico}

\subsection{Sobre el nombre y los rasgos generales de las locuciones:}

El concepto de locución aparece explicado en los manuales analizados como «expresión pluriverbal», «expresión fija», «expresión fraseológica», "combinación de dos o más palabras», etc. Todas estas definiciones coinciden en que se trata de un conjunto de palabras que forman expresiones fijas y estables o, dicho de un modo mejor:

Son unidades léxicas formadas por más de dos palabras gráficas en su límite inferior, cuyo límite superior se sitúa en el nivel de la oración compuesta. Dichas unidades se caracterizan por su alta frecuencia de uso, y de coaparición de sus elementos integrantes; por su institucionalización entendida en términos de fijación y especialización semántica; por su especificidad idiomática; así como por el grado en el cual se dan todos estos aspectos en los distintos tipos. (Corpas Pastor 1996, 20)

Pero ¿cómo sabemos que estamos ante locuciones y no otro tipo de expresiones fraseológicas como las combinaciones libres? Zuluaga $(1980,136)$ explica que mantienen siempre un mismo orden sin que se permita la alteración como en las combinaciones libres; rechazan la inserción de otras palabras que no formen parte de la 
propia combinación, como tampoco permiten separar o modificar el género y número de los propios elementos integrantes. Además, son idiomáticas o semiidiomáticas y esto implica que - por lo generalno se pueden traducir, porque el significado global de la expresión no es la suma de sus componentes.

Las locuciones pueden cumplir la función de diferentes tipos de palabras y, por eso, el Manual de la Nueva gramática de la lengua española $(2010,222-633)$ las clasifica en siete tipos:

- Locuciones nominales: pez gordo

- Locuciones adjetivales: en jarras

- Locuciones verbales: meter la pata

- Locuciones adverbiales: ni más ni menos

- Locuciones prepositivas: a base de

- Locuciones conjuntivas: pese a que

- Locuciones interjectivas: iNi en broma!

\subsection{Clasificación de las locuciones en los distintos manuales}

Para poder determinar cómo se trabajan las locuciones en los manuales, se ha analizado la frecuencia de aparición de cada uno de los tipos descritos anteriormente. De este modo, obtenemos los siguientes porcentajes:

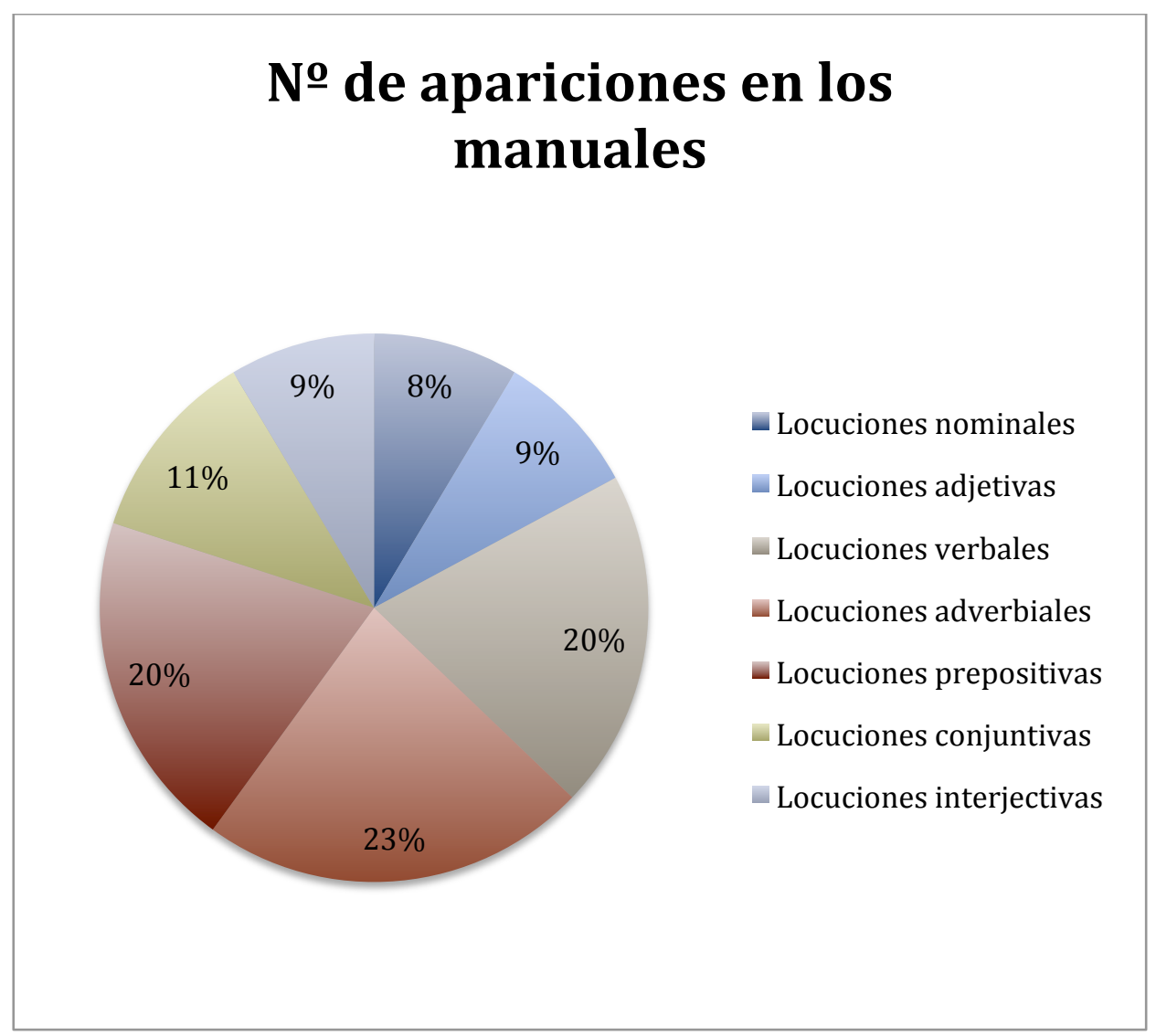

Figura 1. Aparición de los tipos de locuciones (elaboración propia) 
Por lo que respecta a las locuciones nominales, son las menos populares en los manuales didácticos de Bachillerato, dado que tan solo aparecen un $8 \%$ de las veces y, en la mayoría de los casos, se hace de forma general. Esto tal vez se deba a la dificultad que entraña establecer una separación entre los grupos nominales y las locuciones nominales.

Por su parte, las locuciones adjetivas también constituyen un grupo bastante olvidado en los manuales, puesto que apenas llegan al $9 \%$. La razón puede ser la confusión que presenta su forma frente a sintagmas preposicionales de composición libre.

En cambio, las locuciones verbales conforman uno de los grupos más incluidos en los manuales. Además, en buena parte de los casos, las explicaciones de estos sintagmas fijos se ofrecen por oposición a las perífrasis verbales, por lo que resulta mucho más fácil la visualización e identificación de este tipo de locuciones. Se sitúan, junto con las locuciones prepositivas, en un $20 \%$.

Las locuciones adverbiales son las más estudiadas en los manuales ( $23 \%$ ); tal vez sea por la facilidad que presentan a la hora de identificarlas o de sustituirlas por un adverbio.

De igual manera, las locuciones preposicionales se sitúan entre las más explicadas en los manuales con un $20 \%$. Existe una gran variedad de esquemas que forman locuciones prepositivas $y$, además, como se afirma en el manual de la $\operatorname{NGLE}(2010,560)$, "permiten gramaticalizar significados mucho más específicos que los que designan las preposiciones simples».

El grupo de las locuciones conjuntivas, que se incluye junto con las conjunciones, logra alcanzar un $11 \%$ en el análisis.

Finalmente, las locuciones interjectivas se sitúan con un $9 \%$ en el análisis.

Estos porcentajes responden de forma negativa a la pregunta 5 (¿Se dicen todos los tipos de locuciones que existen en la lengua española?) del cuestionario utilizado para el análisis de manuales. A continuación, por ofrecer un ejemplo, vemos cómo ilustra esta cuestión el manual de la editorial Micomicona (2015):

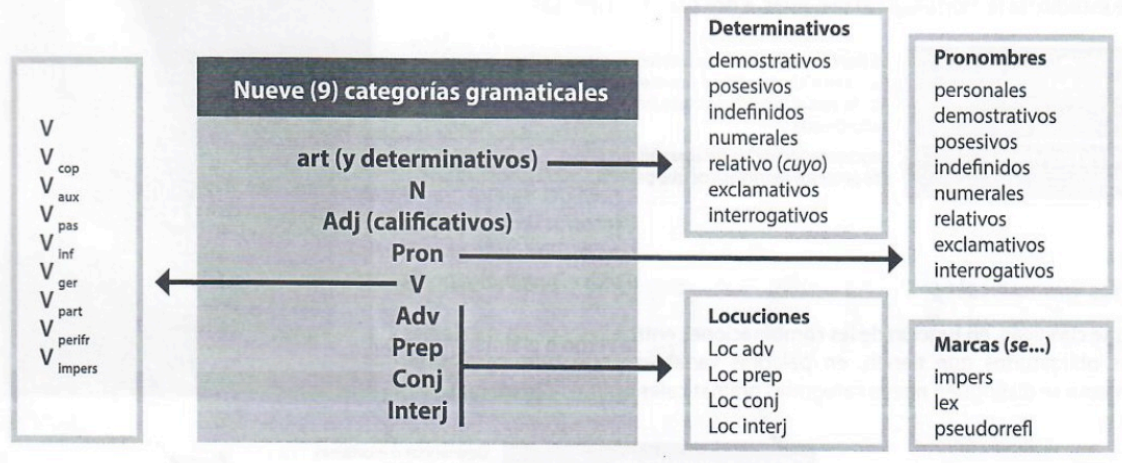

Figura 2. Esquema del manual Micomicona 
Como podemos observar, solo las categorías gramaticales del adverbio, preposición, conjunción e interjección poseen locuciones, mientras que del resto no se dice nada. En definitiva, se trata de un reflejo de lo que encontramos en la mayoría de los manuales, los cuales emplean una clasificación incompleta de las locuciones que se han descrito al inicio de este apartado.

Además, en ninguno de ellos aparece una explicación justificada de por qué se han elegido unas determinadas locuciones y no otras, como tampoco se explica la ausencia de los grupos no tratados. Esto conlleva una confusión a la hora de aprender y reconocer la lengua, porque en los textos no se discrimina la aparición y el uso de unas u otras locuciones. Por lo tanto, la pregunta que nos podemos plantear es la siguiente: ¿por qué a las locuciones no se les concede la misma importancia que al resto de las categorías gramaticales si se encuentran en nuestro día a día?

\subsection{Del uso inconsciente al uso consciente}

El aprendizaje y el uso de las locuciones se adquieren de forma natural e inconsciente. Diversas hipótesis psicolingüísticas como la de Belinchón $(1999,354)$ exponen que los niños empiezan a conocer la estructura del lenguaje a partir de los cuatro años y medio. Al mismo tiempo, «los niños van aprendiendo progresivamente las estructuras fijas de la lengua, y lo hacen del mismo modo que van asimilando otros mecanismos del lenguaje, como las formas flexivas de los verbos» (Ruiz Gurillo 2001, 89).

De esta forma, aprenden las estructuras de un gran número de locuciones y expresiones fraseológicas, como también empiezan a conocer el significado y los contextos en los que se pueden utilizar. Sin embargo, todo esto es producto de la adquisición de la lengua de forma natural y progresiva, pero esto no implica que sepan qué tipo de expresiones están utilizando ni, en un nivel más complejo, las funciones que dichas unidades fraseológicas pueden tener en textos escritos. De hecho, "varias experiencias e investigaciones indican que, para aprender a comunicarnos de manera eficiente, es esencial que las actividades de producción y de recepción de textos de diversos tipos se acompañen de actividades de reflexión sobre los usos mismos» (Fontich 2014, 5).

Por todo ello, las locuciones, como recurso lingüístico que son, deberían introducirse en todos los niveles de la Educación Secundaria, y explicarse con más detalle en Bachillerato. El cambio de un uso inconsciente de las estructuras fraseológicas al uso consciente no es un proceso sencillo; por tanto, previamente, deben trabajarse las bases teóricas para dejar bien definido qué son las locuciones.

Así pues, debemos trabajar en el uso consciente de aquellos recursos que, en el caso de las locuciones, se han adquirido de forma 
inconsciente, para que tengan un sentido y una finalidad dentro del

discurso. De esta manera, saben combinarlas con otras unidades lingüísticas para conseguir el propósito lingüístico que tiene el texto (persuadir, exponer, describir, informar, etc.).

\subsection{Tratamiento a partir de textos}

\subsubsection{Recurso de cohesión y coherencia}

Si partimos del texto como unidad de análisis, observamos la convergencia de diversos fenómenos lingüísticos presentes en la lengua en uso. Aparecen de forma recurrente para que los textos funcionen bien cohesionados y sean coherentes. En este apartado observaremos cómo las locuciones "contribuyen en especial a la cohesión de un texto y, en consecuencia, a proporcionarle su unidad y coherencia» (Ruiz Gurillo 2001, 66).

A continuación, nos serviremos de un ejemplo extraído de la obra de Ruiz Gurillo $(2001,67)$ :

Sara: No, no, no, porque mi novio no se lo merece. Por una parte, ya me ha engañado suficientes veces; por otra parte, nunca tiene ni un detalle conmigo. Sin embargo, es una persona maravillosa con los demás. De todas maneras, no pienso seguir así [...]. Por supuesto que lo quiero, a pesar de que la situación en la que estamos es ya insostenible.

Como vemos en este ejemplo, las locuciones configuran el esqueleto del texto. Podríamos cambiar el propósito de la conversación, pero, si mantenemos las locuciones, veremos que el significado se dirige hacia la misma dirección. Esto nos permite observar que el cuerpo de un texto necesita estar cohesionado gracias a los recursos lingüísticos que intervienen. En esta línea, las locuciones nos ayudan a organizar el mensaje y a extraer la idea de la que se trata en el ejemplo. Al mismo tiempo, estas unidades fijas también se ponen al servicio de los hablantes para satisfacer los propósitos a los que se quiere llegar. Por consiguiente, las locuciones también sirven «como armas argumentativas» (Ruiz Gurillo 2001, 65), porque permiten reforzar las ideas o debilitar los argumentos del otro interlocutor.

En definitiva, nos encontramos ante elementos lingüísticos que pueden trabajarse en el aula como recursos de coherencia y cohesión, como armas argumentativas para reforzar nuestras opiniones, etc. Por lo tanto, para lograr entender su uso lingüístico deben enseñarse dentro de un contexto determinado, dado que pueden adquirir diversas funciones según la finalidad del texto. 


\subsubsection{Recurso expresivo}

El discurso coloquial está repleto de locuciones nominales, adjetivales, verbales, adverbiales e interjectivas, por lo que este puede servir de fuente para el estudio de las locuciones. Como afirma Ruiz Gurillo (2001, 72):

El lenguaje coloquial es [...] una fracción o nivel de lenguaje total que se destaca por su carácter pintoresco reflejado en multitud de expresiones y vocablos intraducibles a otros idiomas, fundados muchas veces en alusiones metafóricas.

Cuando hablamos de expresiones y vocablos intraducibles, nos referimos a unidades fraseológicas como las que se estudian en este trabajo, las cuales son capaces de ocupar el lugar del léxico común. A continuación, presentamos un ejemplo de conversación coloquial que ha sido extraído del corpus del grupo Val.Es.Co:

L: no/ era una chica de quinto/ a mí la verdad es que me importaba muy poco lo que ella hiciera ME DABA igual ¿no? Lo que pasa que me quedé/ un poco de piedra [...].

En este ejemplo, el interlocutor emplea la locución adjetiva de piedra para expresar su sorpresa ante tal situación. A pesar de que no se puede hablar de un léxico estrictamente coloquial, es cierto que aparecen con frecuencia ciertas frases y expresiones como llover a cántaros, libro abierto, no ver tres en un burro, etc. Estos ejemplos nos muestran un registro más relajado, propio del lenguaje coloquial.

Ciertamente, se pueden trabajar las locuciones desde las diferentes tipologías textuales si se conocen las funciones que tienen en cada texto. Por tanto, es necesario abordarlas desde un enfoque comunicativo que nos ayude a comprender su uso pragmático en cada texto. No obstante, se recomienda que se mencionen e integren de forma transversal durante todo el curso a partir de los textos que se estudian en el aula, en vez de tratarlas en una sola unidad didáctica, dado que el uso y la aparición de las locuciones sería más forzoso y artificial.

\section{Metodología del análisis}

\subsection{Selección del corpus recogido}

Este trabajo se basa en el análisis de diez manuales de texto de Lengua Castellana y Literatura utilizados en el curso de 10 de Bachillerato. La selección de este curso para el estudio que se va a realizar se basa en que el alumnado de este nivel debería conocer todas las partes integrantes de las categorías gramaticales. Además, 
el currículo establece que los estudiantes tienen que saber «aplicar sistemáticamente los conocimientos sobre las distintas categorías gramaticales en la realización, autoevaluación y mejora de los textos orales y escritos, tomando conciencia de la importancia del conocimiento gramatical para el uso correcto de la lengua» (Real Decreto 1105/2014).

A continuación, se presenta una tabla que muestra el listado de manuales que configura el corpus:

Tabla 1. Manuales del corpus (elaboración propia)

\begin{tabular}{|cc|}
\hline Editorial & Año \\
\hline Santillana & 2016 \\
\hline Anaya & 2015 \\
\hline Casals & 2015 \\
\hline Editex & 2015 \\
\hline Micomicona & 2015 \\
\hline Oxford & 2015 \\
\hline Sansy & 2015 \\
\hline Teide & 2009 \\
\hline Castellnou & 2008 \\
\hline McGraw-Hill & 2007 \\
\hline
\end{tabular}

Como se observa en la tabla, se han escogido manuales de diferentes editoriales para analizar y comparar el tratamiento de las locuciones en cada uno de ellos. En los apartados siguientes se explica cómo se ha realizado este proceso.

\subsection{Procedimiento de análisis}

La metodología empleada para el análisis de los manuales de texto se lleva a cabo a través de un cuestionario en el que se especifican las preguntas.

Con este cuestionario se puntúa de 0 a 4 la aparición y el tratamiento de las locuciones en todos los manuales. Al mismo tiempo, se han asociado diferentes colores a cada uno de estos valores numéricos. De esta forma, tenemos, por un lado, el análisis cuantitativo que responde al valor numérico que se obtiene de la suma de puntos tras responder a todas las preguntas; por otro lado, los colores vinculados a los números se asocian a un análisis cualitativo.

Posteriormente, se ofrece una tabla para ilustrar la explicación anterior sobre el análisis cuantitativo:

Tabla 2. Análisis cuantitativo (elaboración propia)

\begin{tabular}{|c|c|c|c|c|}
\hline \multicolumn{5}{|c|}{ Análisis cuantitativo } \\
\hline Nunca & Casi nunca & A veces & Casi siempre & Siempre \\
\hline $0 /$ no & 1 & 2 & 3 & $4 /$ sí \\
\hline
\end{tabular}


Respecto al análisis cualitativo, basado en la escala numérica empleada para el procedimiento anterior, se asocia a los siguientes colores:

Tabla 3. Análisis cualitativo (elaboración propia)

\begin{tabular}{|c|c|c|c|c|}
\hline \multicolumn{5}{|c|}{ Análisis cualitativo } \\
\hline Nunca & Casi nunca & A veces & Casi siempre & Siempre \\
\hline $0 /$ no & 1 & 2 & 3 & $4 /$ sí \\
\hline
\end{tabular}

De este modo, al realizar el estudio de cada uno de los manuales de texto, se llevan a cabo ambos análisis. Los valores puntuables se sitúan de forma vertical para poder rellenar — de forma horizontalcada pregunta del cuestionario con el número que le corresponde a la columna rellenada.

\subsection{Cuestionario}

El cuestionario que se presenta a continuación está compuesto por doce preguntas que pretenden desvelar los aspectos más relevantes expuestos en los primeros capítulos de este trabajo.

- Las preguntas 1 y 2 se dirigen a aquellos aspectos más generales que conciernen a la enseñanza de las locuciones dentro de la Programación.

- Las preguntas 3,4 y 5 se centran en conocer qué Contenidos se ofrecen y con qué categorías gramaticales se relacionan.

- Las preguntas $6,7,8$ y 9 estudian cómo se tratan estas unidades en las Actividades y con qué finalidad se emplean.

- Las últimas preguntas, de la 10 a la 12 , se destinan a averiguar si se promueve la Producción de textos y su uso contextualizado.

Veamos ahora el modelo que nos ha servido de plantilla para el análisis: 
Tabla 4. Cuestionario para el análisis (basado en el cuestionario de Blabia 2017)

\begin{tabular}{|c|c|c|c|c|c|c|}
\hline \multirow{2}{*}{\multicolumn{2}{|c|}{$\begin{array}{l}\text { Cuestionario para el análisis de manuales de } \\
\text { texto de Lengua y Literatura Castellana }\end{array}$}} & \multicolumn{5}{|c|}{ Valores puntuables } \\
\hline & & \multirow{2}{*}{$\begin{array}{l}\text { No/ } \\
\text { Nunca }\end{array}$} & \multirow{2}{*}{$\begin{array}{l}\text { Casi } \\
\text { nunca }\end{array}$} & \multirow[t]{2}{*}{ A veces } & \multirow{2}{*}{$\begin{array}{l}\text { Casi } \\
\text { siempre }\end{array}$} & \multirow{2}{*}{$\begin{array}{l}\text { Sí/ } \\
\text { Siempre }\end{array}$} \\
\hline 1 & $\begin{array}{l}\text { Programación: ¿La unidad didáctica en } \\
\text { la que se trabajan las locuciones } \\
\text { presenta una programación horizontal? }\end{array}$ & & & & & \\
\hline 2 & $\begin{array}{l}\text { Programación: ¿Las locuciones están } \\
\text { trabajadas dentro del apartado de } \\
\text { Gramática o Conocimiento de la lengua? }\end{array}$ & & & & & \\
\hline 3 & $\begin{array}{l}\text { Contenidos: ¿Son explicadas como parte } \\
\text { de las categorías gramaticales a las que } \\
\text { hacen referencia? }\end{array}$ & & & & & \\
\hline 4 & $\begin{array}{l}\text { Contenidos: ¿Se ofrece una definición } \\
\text { clara y detallada del concepto de } \\
\text { locución? }\end{array}$ & & & & & \\
\hline 5 & $\begin{array}{l}\text { Contenidos: ¿Se enumeran todas las } \\
\text { clases de locuciones que existen en la } \\
\text { lengua española? }\end{array}$ & & & & & \\
\hline 6 & $\begin{array}{l}\text { Contenidos: ¿Se utilizan ejemplos de } \\
\text { cada tipo para aclarar la definición? }\end{array}$ & & & & & \\
\hline 7 & $\begin{array}{l}\text { Actividades: ¿Aparecen incluidas en } \\
\text { actividades de análisis y comprensión? }\end{array}$ & & & & & \\
\hline 8 & $\begin{array}{l}\text { Actividades: ¿Aparecen incluidas en } \\
\text { actividades de producción? }\end{array}$ & & & & & \\
\hline 9 & $\begin{array}{l}\text { Actividades: ¿Están contextualizadas en } \\
\text { un texto? }\end{array}$ & & & & & \\
\hline 10 & $\begin{array}{l}\text { Producción: ¿Se enseña a utilizarlas en } \\
\text { contextos específicos? }\end{array}$ & & & & & \\
\hline 11 & $\begin{array}{l}\text { Producción: ¿Se promueve su uso en la } \\
\text { producción de textos escritos? }\end{array}$ & & & & & \\
\hline 12 & $\begin{array}{l}\text { Producción: ¿Se promueve su uso en la } \\
\text { producción de textos orales? }\end{array}$ & & & & & \\
\hline
\end{tabular}

Como se ha explicado anteriormente, este es el modelo elaborado para el estudio de los manuales. En él podemos observar tanto el análisis cuantitativo (numérico) como el cualitativo (con colores) que se realiza en cada una de las preguntas.

\section{Resultados}

El análisis de los manuales presentados en el apartado anterior nos ha permitido observar el tratamiento tradicional y escaso que reciben las locuciones en la mayoría de las preguntas presentadas.

Veamos, a partir de la siguiente tabla, cuáles han sido los resultados: 
Tabla 5. Análisis de los resultados

\begin{tabular}{|l|l|l|l|l|l|l|l|l|l|l|l|l|l|}
\hline MANUAL & 1 & 2 & 3 & 4 & 5 & 6 & 7 & 8 & 9 & 10 & 11 & 12 & Total \\
\hline Teide & 0 & 4 & 0 & 4 & 0 & 4 & 3 & 2 & 0 & 0 & 0 & 0 & 17 \\
\hline Sansy & 4 & 4 & 0 & 4 & 4 & 4 & 0 & 0 & 0 & 0 & 0 & 0 & 20 \\
\hline Anaya & 0 & 4 & 4 & 2 & 2 & 4 & 3 & 3 & 2 & 0 & 0 & 0 & 24 \\
\hline Editex & 0 & 4 & 4 & 2 & 1 & 4 & 2 & 0 & 0 & 0 & 0 & 0 & 17 \\
\hline Casals & 4 & 4 & 2 & 2 & 1 & 3 & 2 & 2 & 2 & 0 & 0 & 0 & 22 \\
\hline Micomicona & 0 & 0 & 0 & 0 & 0 & 0 & 0 & 0 & 0 & 0 & 0 & 0 & 0 \\
\hline Oxford & 0 & 4 & 4 & 4 & 3 & 4 & 1 & 0 & 0 & 0 & 0 & 0 & 20 \\
\hline McGraw-Hill & 0 & 4 & 2 & 0 & 1 & 4 & 0 & 2 & 0 & 0 & 0 & 0 & 13 \\
\hline Castellnou & 0 & 4 & 2 & 0 & 1 & 4 & 1 & 0 & 0 & 0 & 0 & 0 & 12 \\
\hline Santillana & 0 & 0 & 0 & 0 & 0 & 0 & 0 & 0 & 0 & 0 & 0 & 0 & 0 \\
\hline Total & 8 & 32 & 18 & 18 & 13 & 31 & 12 & 9 & 4 & 0 & 0 & 0 & \\
\hline
\end{tabular}

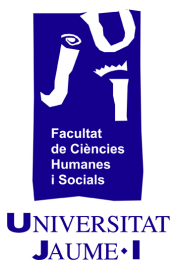

Escala de colores para la puntuación:

0 puntos: No/ Nunca

1 punto: Casi nunca

2 puntos: A veces

3 puntos: Casi siempre

4 puntos: Sí / Siempre

Como se puede observar en la tabla, si empezamos por la parte práctica, lo más destacado es la ausencia de tareas destinadas a la producción (preguntas 10, 11 y 12). En cuanto a las actividades (preguntas 7,8 y 9), estas se integran escasamente entre los ejercicios de las unidades donde se trabajan estas expresiones fijas. Por último, las primeras preguntas (de la 1 a la 6), de carácter más teórico, aluden a la Programación y Contenidos, y parece ser que sí ofrecen con mayor claridad la información que se pide. Incluso así, si nos fijamos en la primera pregunta - referida a la programación en horizontal-, veremos que la mayoría de los manuales carecen de este tipo de programación, la cual es necesaria para el desarrollo de un enfoque comunicativo dentro de las unidades.

\subsection{Tipo de programación}

La mayor parte de los manuales responden con un 'no' a la primera cuestión, referida a la estructuración en horizontal de la materia curricular. Aquellos que han contestado de forma negativa a esta cuestión distribuyen el contenido de la materia de forma vertical; en consecuencia, no se establece conexión entre el contenido lingüístico y el texto de la unidad. Consecuentemente, se observa una falta de contextualización y relación dentro de los textos y los recursos que en ellos aparecen. Así pues, la explicación de las funciones de las locuciones carece de sentido por no ofrecer una aplicación pragmática. 
Además, la disposición de la materia curricular está dividida en compartimentos estancos, separados y sin conexión alguna entre ellos, por lo que es casi imposible llevar un enfoque comunicativo a lo largo de estas programaciones y, por tanto, no se trabajan las locuciones contextualizadas.

La segunda cuestión consiste en conocer la ubicación de las locuciones dentro del Conocimiento de la lengua o, si el manual es más antiguo, en la Gramática. En los manuales más actuales hay más cohesión y sistematicidad a la hora de incluirlos dentro de este apartado, mientras que en aquellos que tienen una programación más tradicional, existe mayor variedad a la hora de situarlas entre Gramática y Léxico. Sin embargo, la clasificación en un apartado u otro no encuentra su correspondencia en las actividades, es decir, aquellos manuales que las sitúan en el apartado gramatical deberían hacer énfasis en la función de las locuciones como recursos argumentativos y de coherencia y cohesión, mientras que los manuales que las sitúan en el apartado del léxico tendrían que desarrollar con mayor amplitud sus funciones como recursos expresivos.

Incluso así, en este aspecto el resultado general es favorable, dado que la ubicación de la mayoría de ellas se recogen en el Conocimiento de la lengua $\mathrm{y}$, por consiguiente, esto posibilita el tratamiento de las locuciones en todas sus vertientes.

\subsection{Contenidos y actividades}

Las cuestiones de la 3 a la 6 pretenden indagar sobre cómo se ha establecido y explicado la base teórica de las locuciones.

La tercera cuestión se refiere a la inclusión de estas dentro de las categorías gramaticales a las que pertenecen: el proceso de análisis muestra de forma sorprendente las clasificaciones incompletas en la mayoría de los manuales.

En cuanto a la siguiente cuestión, referida a la definición del término locución (pregunta 4), siguen siendo la minoría los manuales que establecen las bases teóricas de forma clara para identificarlas y reconocer de qué tipo se trata. Consecuentemente, esta confusión concluye, como ya se ha comentado, en la presentación de una clasificación incompleta y poco objetiva.

Por último, la pregunta 6 se refiere al uso de ejemplos para mejorar la comprensión de las definiciones. En la mayoría de los casos, los resultados han sido afirmativos y ofrecen un listado de ejemplos que acompañan la definición.

En cuanto a las actividades, las cuestiones destinadas a ellas son las siguientes: 7,8 y 9.

La primera de ellas, la pregunta 7 , intenta descubrir si las actividades propuestas tienen como trasfondo el análisis y la comprensión de la teoría explicada anteriormente. Pero, como se 
observa en la tabla de resultados, son pocos los ejercicios que trabajan este aspecto.

A continuación, las siguientes actividades, la 8 y 9 , son prácticamente inexistentes en todos los manuales analizados. Como vemos en la tabla que resume los resultados, la mayoría de ellos responde de forma negativa a la motivación de promover actividades que desarrollen y potencien la producción de textos y ejercicios que impliquen una mínima contextualización de las locuciones.

\subsection{Producción}

Finalmente, de la cuestión 10 a la 12 se examina qué lugar se otorga a las locuciones dentro del texto cuando se proponen actividades de producción y si se promueve su uso como recursos lingüísticos en la elaboración de textos.

La cuestión número 10 pregunta: "¿Se enseña a utilizarlas en contextos específicos?». Todos los manuales responden con un 'no'. Consecuentemente, la intención didáctica que se esconde tras los contenidos queda incompleta por la ausencia de su aplicación en un contexto determinado. Esto también está ligado a la negación que se ha obtenido en la primera pregunta, es decir, si no se aplica una programación horizontal, las unidades probablemente carecen de un enfoque comunicativo y, en consecuencia, desaparece la motivación de la producción de textos, tanto orales -como se pregunta en la cuestión 11-, como escritos - cuestión 12-.

De estos datos se desprende que este es uno de los grandes aspectos que se debería fortalecer a la hora de incluir la enseñanza de las locuciones en un contexto según un enfoque comunicativo, dado que si solo se explica la teoría, pero no se trabajan los aspectos prácticos, el proceso de asimilación y comprensión de estos recursos lingüísticos queda incompleto.

\section{Conclusiones}

Tras el análisis de los manuales, se concluye que las locuciones son recursos lingüísticos que no se potencian en las clases de Secundaria y, en concreto, en 10 de Bachillerato.

El principal error recae en el tratamiento que reciben en los manuales, dado que se aíslan en cuadros situados en los laterales de las páginas como información secundaria $y$, además, nunca se relacionan con las actividades que se proponen en la unidad donde aparecen. Asimismo, no se presentan de forma sistemática y objetiva porque no se describen todas las subclases que se exponen en el presente trabajo.

Se quiere demostrar también que una mayor atención a las locuciones en la lengua materna de los alumnos posibilitaría un aumento de recursos lingüísticos a la hora de expresarse y, por tanto, 
una mayor competencia comunicativa. Es más, este proceso de

enseñanza que vincula las locuciones con otros elementos lingüísticos permite tanto la reflexión sobre el conocimiento de la lengua, como el desarrollo de las destrezas discursivas más inmediatas en su día a día.

\section{VIII.Bibliografía}

Belinchón Carmona, Mercedes. 1999. «Lenguaje no literal y aspectos pragmáticos de la comprensión». Psicolingüística del español, coordinado por Fernando Cuetos Vega y Manuel de Vega Rodríguez, 307-373. Madrid: Trotta.

Blabia Girau, Irene. 2017. ¿Usuarios o sabios de los textos periodísticos informativos?. Trabajo de Fin de Máster. Castelló de la Plana: Publicacions de la Universitat Jaume I.

Briz, Antonio. Grupo Val.Es.Co. 1995. "La conversación coloquial. Materiales para su estudio». Cuadernos de Filología 16: 175-189.

Bronckart, Jean Paul y Bernard Schneuwly. 1996. "La didáctica de la lengua materna: el nacimiento de una utopía indispensable». Textos de Didáctica de la Lengua y la Literatura 9: 61-79.

Coronas Peñarrocha, Ricardo. 2010. «Las oraciones coordinadas en los libros de texto de lengua castellana». En Libros de texto y enseñanza de la gramática, coordinado por Teresa Ribas, 117134. Barcelona: Graó.

Corpas Pastor, Gloria. 1996. Manual de fraseología español. Madrid: Gredos.

Coseriu, Eugenio. 1977. Estudios de lingüística románica. Madrid: Gredos.

Fontich, Xavier. 2014. «La enseñanza de la gramática en la educación secundaria». Didáctica de la Lengua y de la Literatura, Textos 67: 5-6.

Forment Fernández, Mạ del Mar. 1997. "La didáctica de la fraseología ayer y hoy: del aprendizaje memorístico al agrupamiento en los repertorios de funciones comunicativas». Centro Virtual Cervantes 8: 339-347.

García Muruais Ma Teresa. 1997. «Propuesta para la enseñanza de unidades fraseológicas en a clase de E/LE». Centro Virtual Cervantes 8: 363-369.

Gómez González, Alba y Clara Ureña Tormo. 2015. Locuciones y refranes para dar y toma. El libro para aprender más de 120 locuciones y refranes en español. Niveles B2 y C1. Alcalá de Henares: Publicaciones de la Universidad de Alcalá. 
Iliná, Natalia. 2000. «Fraseología española contemporánea: estado de la cuestión». Actas de la II Conferencia de hispanistas de Rusia, vol. 4, 207-218. Moscú: Facultad de Lenguas Extranjeras adjunta a la Universidad Estatal Lomonosov.

Lamas, Óscar Loureda. 1999. "Acerca del objeto y los objetivos de la enseñanza del Área de Lengua y Literatura en la Enseñanza Secundaria Obligatoria». Rilce 15 (2): 427-438.

Ley Orgánica 1105/2014, de 26 de diciembre, para la mejora de la calidad educativa. Boletín Oficial del Estado 3: 169-543.

Lomas, Carlos. 2003. "Aprender a comunicar(se) en las aulas». Ágora Digital 5: 1-17.

Martínez Marín, Juan. 2000. "Las unidades léxicas complejas en español: aspectos teóricos y descriptivos». Revista de Investigación Lingüística 3: 315-338.

Núñez-Román, Francisco. 2015. «Enseñar fraseología: consideraciones sobre la fraseodidáctica del español». Didáctica: Lengua y Literatura 27: 153-166.

Real Academia Española. 2010. Nueva gramática de la lengua española. Manual, Madrid: Espasa Calpe.

Ruiz Gurillo, Leonor. 2001. Las locuciones en el español actual. Madrid: Arcos.

Zuluaga, Alberto. 1980. «Introducción al estudio de las expresiones fijas». Studia Romanica et Lingüística 10: 126-141. 


\section{Estudis de Traducció i Interpretació}

Estudios de Traducción e Interpretación Translation and Interpreting Studies 



\section{Problemas de comunicación intercultural en salud sexual y reproductiva}

Análisis de los principales problemas que experimentan las pacientes de origen chino en España

María López Rubio

marialopezrubi@hotmail.com 
I. Resumen

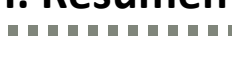

España experimenta un importante incremento en el flujo de inmigración desde finales del siglo $x x y$, como resultado, se ha producido un importante incremento en el número de nacimientos de madres de origen extranjero. No obstante, este auge no ha venido acompañado de mejoras para conseguir que se respeten los derechos de cualquier ciudadano en los servicios sanitarios. Pese a tener las mismas obligaciones que las mujeres autóctonas, las mujeres de origen extranjero que esperan ser madres en España se encuentran con grandes limitaciones a la hora de ejercer sus derechos en este campo debido a los obstáculos que encuentran para la comunicación. Mediante el presente estudio se pretende mostrar los diferentes problemas interculturales que se producen en la interacción médico-paciente en los hospitales y centros de salud públicos de la Comunidad Valenciana. A partir de los resultados obtenidos de las entrevistas y los grupos de discusión con pacientes de origen chino y personal sociosanitario, se pretende identificar los problemas de comunicación intercultural que mayor incidencia tienen en la atención recibida por las pacientes de origen chino en el ámbito de la salud sexual y reproductiva y cómo podría mejorarse la situación. El resultado del análisis ha puesto de manifiesto que la visión de ambos colectivos en cuanto a la atención en salud sexual y reproductiva es muy distinta, pues son los profesionales quienes más problemas encuentran en este ámbito, especialmente problemas de cultura general, problemas relacionados con los patrones de interacción comunicativa, problemas de comunicación lingüística y problemas administrativos y laborales.

Palabras clave: pacientes de origen chino, personal sociosanitario español, problemas de comunicación intercultural, atención sanitaria en España, salud sexual y reproductiva.

\section{Introducción}

Las pacientes de origen inmigrante se encuentran con muchos problemas comunicativos a la hora de ejercer sus derechos de acceso a los servicios sanitarios españoles, especialmente en el ámbito de la salud sexual y reproductiva, pues es una especialidad que está muy ligada a la realidad cultural de cada país. Estos problemas se acentúan notablemente cuando la población extranjera de llegada es de una cultura muy diferente a la de las pacientes. Los problemas de comunicación suelen derivar en malentendidos, desconfianza o discusiones, y en cualquiera de los casos tienen consecuencias muy perjudiciales para la salud de las pacientes. El principal motivo por el 
que he escogido la población china es precisamente que se trata de una comunidad cuya tradición cultural es muy diferente a la española. Además, he decidido centrarme en el campo de la salud sexual y reproductiva porque, a diferencia de otras disciplinas como la traumatología, la SSyR es una rama de especialización muy sensible desde el punto de vista personal y cultural, en la que se tratan temas como la maternidad o la sexualidad, que están muy marcados culturalmente.

\section{Objetivos}

El propósito principal de esta investigación es analizar los principales problemas de comunicación que surgen a raíz de la interacción entre las pacientes de origen chino y los miembros del personal sociosanitario español.

A continuación, se presentan los objetivos específicos del estudio que realizamos:

- Analizar la relevancia de los problemas para proveer una atención sanitaria adecuada.

- Evaluar, a modo de conclusiones, la incidencia de los problemas interculturales detectados y observar si se vulneran o no los derechos de las pacientes de origen chino.

\section{Metodología y tablas de análisis de problemas}

\subsection{Metodología}

La metodología que se ha seguido para elaborar este trabajo es de corte descriptivo, ya que se busca especificar algunas de las propiedades más importantes de la atención que reciben las pacientes de origen chino en salud sexual y reproductiva en España a partir de las experiencias compartidas por las propias pacientes de origen chino y los miembros del personal sociosanitario que prestan atención sanitaria durante el embarazo, el parto, el posparto y la atención en pediatría.

Si bien resulta difícil establecer una clasificación sistemática de los elementos que componen la cultura, el grupo CRIT (Raga Gimeno $2007,15)$ ha establecido que, por un lado, se puede hablar de problemas relacionados con los patrones de interacción comunicativa (PIC) y, por otro, de aspectos de cultura general. A partir de los datos obtenidos y del contraste entre los resultados de los grupos de discusión y las entrevistas realizadas a las pacientes y a los profesionales tomando como base algunas de las cuestiones que plantea Sánchez Pérez (2009, 110-112), se han detectado y descrito 
los principales problemas de comunicación derivados de las

diferencias interculturales.

\subsection{Problemas observados por las pacientes de origen chino en el grupo de discusión}

Tabla 1. Problemas observados por las pacientes de origen chino en el grupo de discusión. Fuente: Elaboración propia.

\begin{tabular}{|c|}
\hline Problemas de cultura general \\
\hline $\begin{array}{l}\text { Desconocimiento de los cursillos de preparación al parto. } \\
\text { Pérdida de contacto con las costumbres del país de origen. } \\
\text { Posible contraindicación de los tratamientos de la MTC. } \\
\text { Consideración del embarazo como un estado de salud. }\end{array}$ \\
\hline Problemas relacionados con los PIC \\
\hline $\begin{array}{l}\text { Gesticulación como obstáculo vs. gesticulación como recurso comunicativo. } \\
\text { Algunos profesionales no miran a las pacientes a los ojos. } \\
\text { Algunos profesionales hablan rápido y no dan muchas explicaciones. }\end{array}$ \\
\hline Problemas de comunicación lingüística \\
\hline $\begin{array}{l}\text { Desconocimiento del idioma. } \\
\text { Dificultad para entenderse cuando se trata de enfermedades mayores. } \\
\text { Falta de servicios de mediación intercultural. }\end{array}$ \\
\hline Problemas administrativos y laborales \\
\hline $\begin{array}{l}\text { Necesidad de eliminar o reducir el periodo de reposo por motivos laborales. } \\
\text { Falta de tiempo para asistir a los cursillos. } \\
\text { Desconocimiento de la forma de proceder de los servicios sanitarios españoles. }\end{array}$ \\
\hline
\end{tabular}

\subsection{Problemas observados por los miembros del personal sociosanitario en el grupo de discusión}

Tabla 2. Problemas observados por los integrantes de los GD con profesionales del centro de salud y el hospital de la Comunidad Valenciana. Fuente: Elaboración propia.

\begin{tabular}{|l|}
\hline \multicolumn{1}{|c|}{ Problemas de cultura general } \\
\hline Baja asistencia de las pacientes a los servicios sanitarios. \\
Tendencia a acudir a múltiples especialistas. \\
Desconocimiento de la organización de los servicios sanitarios en España. \\
Las pocas pacientes que practican la lactancia lo hacen de forma aleatoria. \\
Posible contraindicación de la MTC con la medicina occidental. \\
Desconocimiento de las prácticas de la MTC por parte de los profesionales. \\
\hline
\end{tabular}


Dificultad para conocer los componentes de la MTC.

Alto índice de niños sobrealimentados.

Posibilidades de comunicarse reducidas.

Escasa atención que, en general, las pacientes prestan al bebé.

\section{Problemas relacionados con los PIC}

Dificultad para entender ciertas formas de actuar (p. ej. el uso de la sonrisa).

Las pacientes tienden a responder con brevedad a las preguntas.

Dificultad para saber si las pacientes los entienden o si les dicen la verdad.

Algunas pacientes no escuchan o no entienden y vuelven preocupadas.

Las pacientes tienden a mostrarse cohibidas y poco participativas.

Actitud menos cálida y más resignada que la de las pacientes autóctonas.

Los profesionales no llegan a transmitir lo que desearían.

Las madres se muestran tensas ante la exploración de los niños.

Las pacientes podrían estar demasiado pendientes de su acompañante.

Escasez de preguntas planteadas por las pacientes.

\section{Problemas de comunicación lingüística}

Las pacientes interpretan las palabras desde su realidad cultural.

Esfuerzo de los profesionales por hacerse entender.

Rechazo de métodos como la interpretación telefónica.

Posibilidad de que los niños que traducen no se tomen en serio ciertos temas.

Mayor presencia de la traducción escrita por motivos de responsabilidad legal.

La autonomía de las pacientes se ve mermada.

Presencia de intérpretes ad hoc en lugar intérpretes formados.

Pérdida de información.

\section{Problemas administrativos y laborales}

Dificultad para guardar reposo durante y después del embarazo.

Los centros de salud y hospitales no cuentan con servicios de mediación.

Falta de tiempo de las pacientes para asistir a los cursillos.

Asistencia a pediatría solo cuando es estrictamente necesario.

Dificultad para llevar un seguimiento de los pacientes en pediatría. 


\subsection{Problemas observados por las pacientes de origen chino en las entrevistas}

Tabla 3. Problemas observados por las pacientes de origen chino entrevistadas. Fuente: Elaboración propia

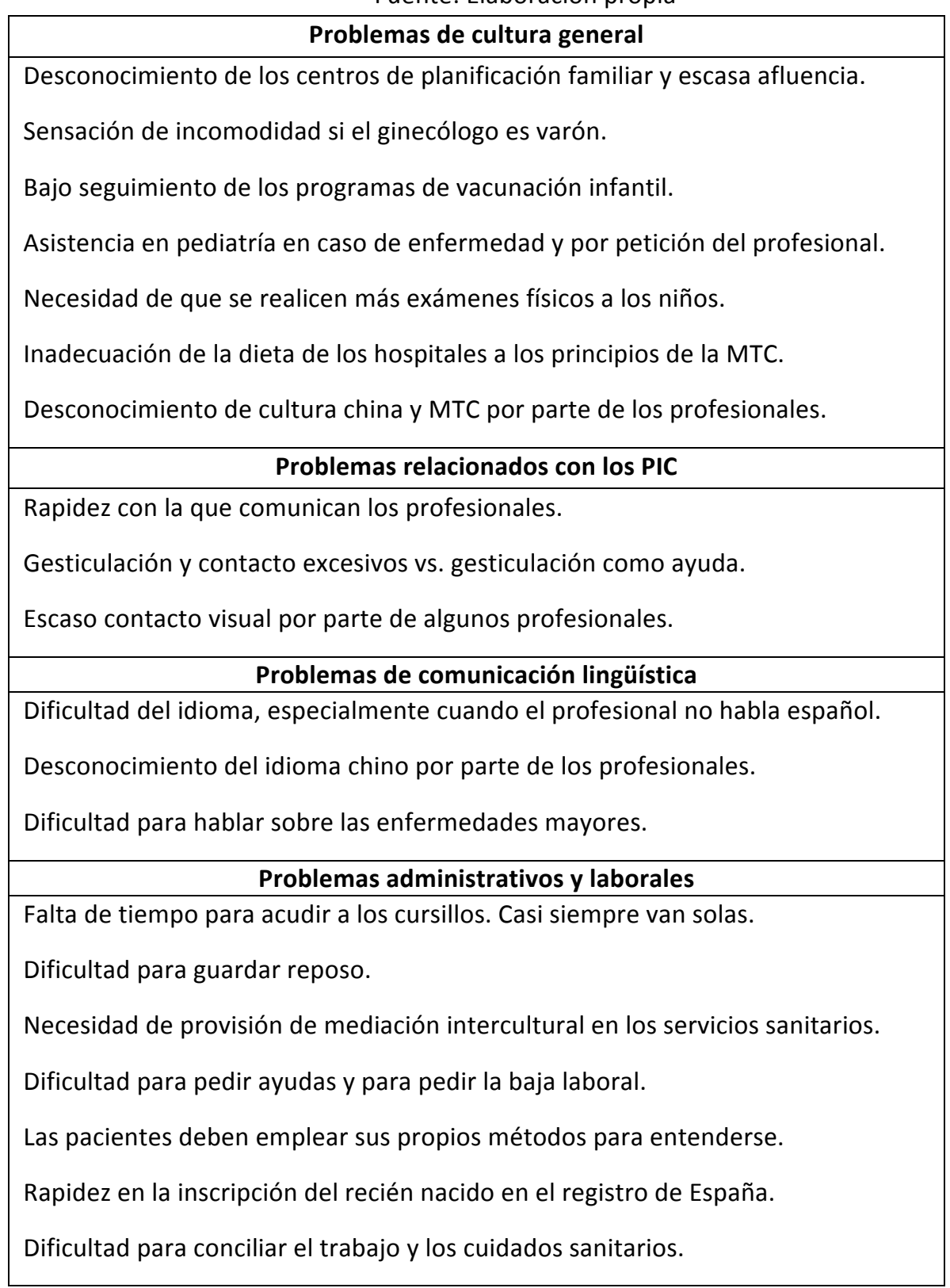




\subsection{Problemas observados por los miembros del personal sociosanitario en las entrevistas}

Tabla 4. Problemas observados por los profesionales entrevistados del centro de salud y el hospital de la Comunidad Valenciana. Fuente: Elaboración propia

\begin{tabular}{|c|}
\hline Problemas de cultura general \\
\hline $\begin{array}{l}\text { Escasa afluencia a los servicios sanitarios. } \\
\text { Dificultad para saber la composición y posibles contraindicaciones de la MTC. } \\
\text { Escasas explicaciones sobre los tratamientos de la MTC. } \\
\text { Alto índice de niños de origen chino con sobrepeso. } \\
\text { Tendencia a interpretar lo que les ocurre desde su propia realidad cultural. }\end{array}$ \\
\hline Problemas relacionados con los PIC \\
\hline $\begin{array}{l}\text { Tendencia a decir que sí cuando les preguntan, aunque no presten atención. } \\
\text { Distinta interpretación de la sonrisa en ambas culturas. } \\
\text { Tendencia a no expresar sus preocupaciones ni plantear sus dudas. } \\
\text { Situación tensa de las madres durante la exploración de los niños. } \\
\text { Tendencia a responder de forma breve y solo cuando se les pregunta o insiste. } \\
\text { Generalmente las pacientes se muestran introvertidas y poco cálidas. }\end{array}$ \\
\hline Problemas de comunicación lingüística \\
\hline $\begin{array}{l}\text { Dificultad para comunicarse en una lengua compartida. } \\
\text { Comprensión de las palabras desde el marco de significación de su cultura. } \\
\text { Desprotección de las pacientes al no entender el idioma y qué les ocurre. } \\
\text { Esfuerzo de los profesionales por transmitir matices y asegurar la comprensión. } \\
\text { Se pierde información y matices con la interpretación ad hoc. } \\
\text { Los familiares no están preparados para ser mediadores, sobre todo los niños. } \\
\text { Necesidad de hablar fluido en especialidades como la psicología en SSyR. }\end{array}$ \\
\hline Problemas administrativos y laborales \\
\hline $\begin{array}{l}\text { Posible incumplimiento de las normas del hospital por traer alimentos externos. } \\
\text { Necesidad de contar con mediadores interculturales. } \\
\text { Dificultad para entender los trámites administrativos. }\end{array}$ \\
\hline
\end{tabular}

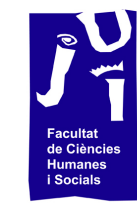

\section{Resultados}

Una vez enunciados los principales problemas encontrados por los profesionales y las pacientes, se comparan ambas perspectivas partiendo de las cuestiones de cultura general, los PIC, la comunicación y las cuestiones administrativas y laborales mediante el gráfico siguiente: 


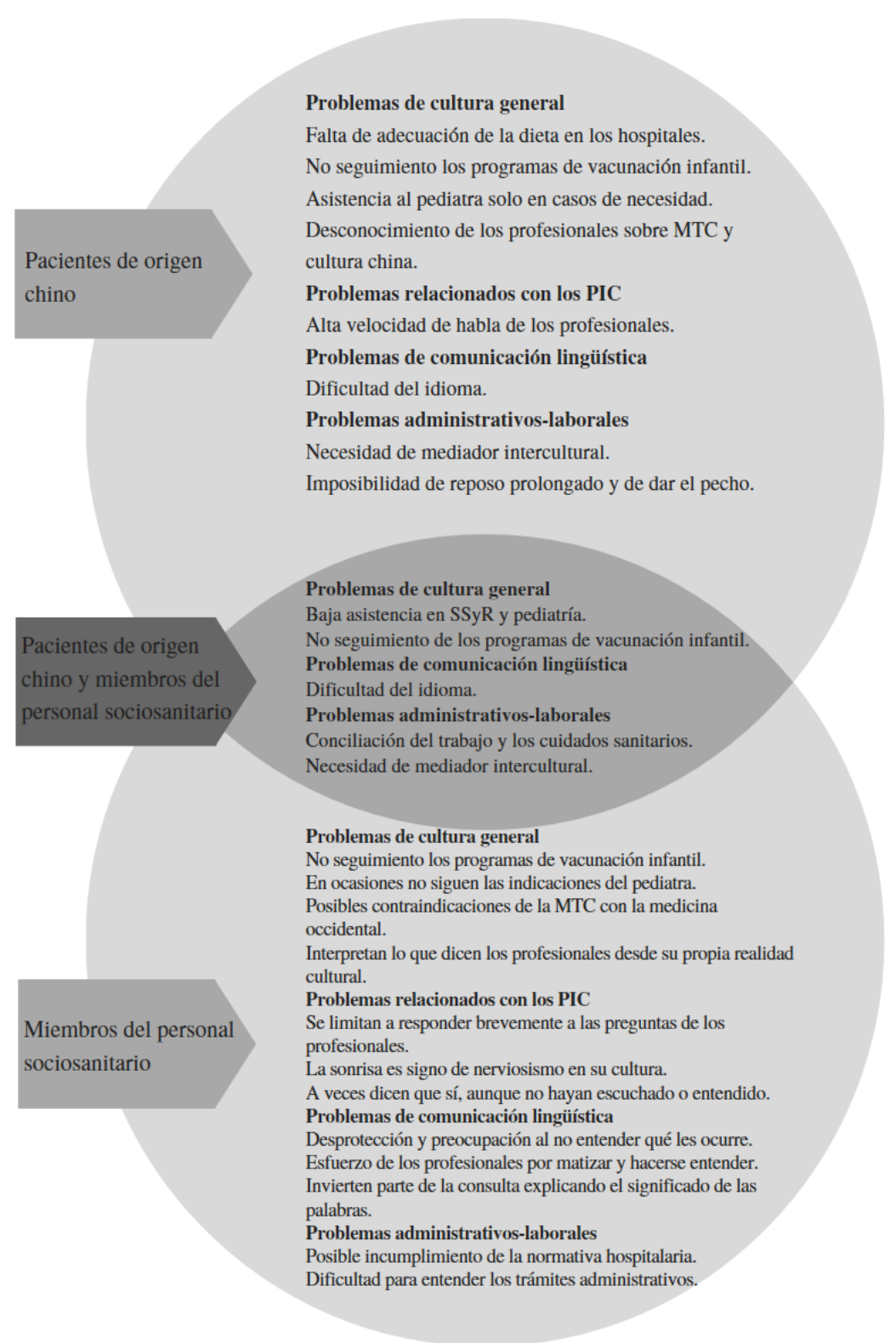

as de cultura general

cultura china.

Problemas relacionados con los PIC

Alta velocidad de habla de los profesionales.

de dar el pecho.

Gráfico 1. Comparación de los principales problemas observados por los miembros del personal sociosanitario y las pacientes de origen chino. Fuente: Elaboración propia

\section{Discusión y conclusiones}

A continuación, extraemos las conclusiones derivadas del análisis descriptivo y cualitativo que se recoge en el capítulo anterior. En el contexto sanitario, la importancia de comunicar correctamente el mensaje que se desea transmitir es aún mayor porque de ello depende muchas veces la salud de los y las pacientes. En palabras de Ho Wong $(2009,10)$, en un encuentro intercultural «el idioma en su forma verbal es la diferencia más destacada y el obstáculo que más afecta en primera instancia a la interacción paciente-profesional y viceversa», si bien, como demuestra el estudio, el idioma no es el único obstáculo con el que se encuentran los pacientes y los 
profesionales. Lograr que la comunicación sea efectiva cuando el interlocutor se inscribe en una cultura diferente y habla una lengua distinta a la de la población autóctona supone una de las grandes luchas que se llevan a cabo en el panorama sanitario de nuestro país en la actualidad. Resulta conveniente, pues, destacar el interés que sugiere el estudio de un colectivo de pacientes tan alejado culturalmente como es la población de origen chino, ya que, a pesar de la escasa afluencia que registran en los servicios sanitarios $y$, en especial, en el ámbito de la SSyR, cuando acuden a la sanidad española se encuentran con numerosos problemas de comunicación.

Por otro lado, la figura de la MILICS (intérprete en servicios sanitarios y mediador intercultural) ha estado más presente a lo largo del estudio de lo que en un principio había previsto. La mayoría de las pacientes de origen chino y todos los miembros del personal sociosanitario defienden la necesidad de disponer de servicios de mediación, cuyos representantes cumpliesen, de acuerdo con Roberts $(1997,13)$, su labor de «defending, pleading for or actively supporting the client. In other words, the community interpreter is seen as a guide and counsellor as well as a power broker working in favour of his 'underprivileged' client». Además de la labor de los mediadores interculturales, otro recurso que, de acuerdo con los resultados del estudio ha demostrado tener una gran efectividad a la hora de facilitar la comunicación entre el personal sociosanitario y las pacientes de origen chino es el empleo de herramientas informáticas como el traductor de Google.

Tal y como se ha comprobado con la elaboración de este trabajo, los problemas relacionados con los PIC y con la cultura en general influyen en gran medida en la atención que reciben las pacientes, si bien se producen también otros problemas de tipo administrativo y laboral y de comunicación lingüística que conviene tener en cuenta. En lo referente a las cuestiones que mayor incidencia tienen sobre la atención que reciben las pacientes de origen chino, las exigencias laborales tienen un peso importante en el acceso de estas pacientes a los servicios sanitarios en general y al ámbito de la SSyR en particular.

En cualquier caso, los patrones de comportamiento observados en las participantes del estudio no se pueden extrapolar a todas las pacientes de origen chino, por muy amplia que sea la muestra analizada, ya que de todos es sabido que no existen dos personas iguales. No se puede establecer una guía que sirva al personal sociosanitario para identificar el comportamiento de cada paciente de origen chino, porque cada paciente, sea del país que sea, actuará de manera distinta a otro con quien comparta los mismos patrones de comunicación. 
Por lo que a perspectivas de futuro respecta, conviene destacar que, durante el proceso de elaboración de este trabajo, se han tomado principalmente dos vertientes de estudio, la población de origen chino y la atención sanitaria en SSyR, que podrían dar lugar a investigaciones o ampliaciones en el futuro.

Por un lado, existe la posibilidad de ahondar más en este campo de investigación tomando como referencia otras poblaciones extranjeras que presentan importantes diferencias culturales respecto a la población de acogida, como la población magrebí o la senegalesa. Otra cuestión que suscitaría mucho interés es analizar los problemas que se producen en otras áreas del sector sanitario también marcadas culturalmente, como es el caso de la salud mental. Asimismo, tal y como establece Sánchez Pérez $(2015,313)$, resultaría relevante indagar en el subámbito de la endocrinología, ya que la necesidad de seguir las pautas alimenticias que dicta la MTC en ocasiones entra en conflicto con las prescripciones del personal sociosanitario español.

Sería interesante, además, recoger la opinión de más participantes en el marco de las entrevistas y de los GD con pacientes de origen chino y miembros del personal sociosanitario especialistas en SSyR, de modo que los resultados representen de forma más fiel la visión de cada colectivo estudiado.

Un objeto de estudio que podría contemplarse para futuros trabajos es el de las técnicas y estrategias para la resolución de los problemas que se enuncian a lo largo del trabajo. Durante esta investigación se ha observado que tanto los miembros del personal sociosanitario español como las pacientes de origen chino emplean todos los recursos que tienen a su disposición con el fin de facilitar el entendimiento mutuo.

Por otro lado, puesto que todos los participantes del estudio coinciden en la importancia que tiene el/la MILICS en la interacción médico-paciente, otra posible vía de estudio que sigue por la línea de esta investigación es analizar el rol que el/la MILICS desempeña en la atención sanitaria prestada a las pacientes de origen chino en SSyR. Sería interesante plantear al/a la MILICS las cuestiones relacionadas con los PIC y con la cultura general que, entre otras, se han considerado a lo largo de este trabajo, de modo que se pueda analizar la situación en el sector sanitario desde la perspectiva de este tercer colectivo social, cuyo protagonismo en nuestro país es muy limitado a día de hoy.

Por último, suscitaría un gran interés para la investigación ampliar los resultados obtenidos ejerciendo como MILICS en una consulta $y$, por tanto, analizando mediante la observación participante la interacción entre profesionales del ámbito de la SSyR 
y pacientes de origen chino. De esa forma es posible que se observasen diferencias entre los PIC que podrían desencadenar problemas, ya que los PIC comprenden rasgos sutiles que pueden atenuarse con el tiempo o pasar desapercibidos para los participantes en el acto de comunicación, pero aun así tienen consecuencias notables en la atención sanitaria y en la impresión que ambos interlocutores reciben durante la interacción comunicativa, tal y como se ha podido observar a lo largo de este estudio.

\section{Bibliografía}

Ho Wong, Tai Mooi. 2009. "Capítulo 24: La salud en los pacientes chinos». En Mediación intercultural en el ámbito de la salud: Programa de formación, editado por Miquel Casas, Francisco Collazos y Adil Qureshi, 1-16. Barcelona: Fundación La Caixa. Acceso:

https://multimedia.caixabank.es/lacaixa/ondemand/obrasocial/ pdf/inmigracion/Mediacio_intercultural_es.pdf.

Raga Gimeno, Francisco. 2007. «Mediaciones interculturales». Acceso http://www.segundaslenguaseinmigracion.com/ense_anzal2/M ediacioninterculutralRAGA.pdf.

Roberts, Roda P. 1997. "Community interpreting today and tomorrow». En The Critical Link: Interpreters in the Community, editado por Silvana E. Carr, Roda P. Roberts, Aideen Dufour y Dini Steyn, 7-26. Ámsterdam/Filadelfia: John Benjamins.

Sánchez Pérez, Marta. 2009. La mediación intercultural en el ámbito sanitario. El caso de los pacientes de origen chino. Trabajo Final de Máster. Castelló de la Plana: Universitat Jaume I. Acceso: http://www.saludycultura.uji.es/archivos/La_mediacion_intercul tural_en_el_ambito_sanitario_El_caso_de_los_pacientes_de_ori gen_chino.pdf. 



\section{Traducción feminista:}

conciencia de género, intervencionismo y estrategias

Pilar Querol Pérez

al314200@uji.es 
I. Resumen

\section{Resumen}

Este trabajo parte de la base de que existe una relación entre lenguaje y género de la que la traductora ha de ser consciente cuando traduce un texto. Haciendo uso de esta conciencia se puede percibir que el lenguaje no se emplea de forma paritaria, sino que es esencialmente patriarcal y a través de este uso abusivo se han conformado las estructuras sociales y políticas vigentes hoy en día que se caracterizan por sus incontables argucias para invisibilizar y excluir a las mujeres.

Una de las formas mediante las que puede subsanarse esta concepción de superioridad lingüística masculina es la traducción feminista, el punto de convergencia entre feminismo y traducción y concepto en torno al que gira mi trabajo. Esta práctica traductora supone una manipulación deliberada del texto meta con el objetivo de que las mujeres por fin puedan sentirse representadas a través del lenguaje y se apoya en la idea de que deconstruir el sexismo lingüístico significa deconstruir el sexismo sociopolítico.

Sin embargo, esta práctica es intrépida debido a su falta de normalización y en la mayoría de ocasiones se tildará a la traductora de infiel y subversiva por las técnicas y estrategias de las que haga uso para alcanzar sus objetivos.

Este trabajo no es un análisis traductológico al uso; se trata más bien de una exploración de las cuestiones que me han resultado más sugestivas y destacables acerca de la traducción feminista a medida que he ido nutriendo y ampliando mis conocimientos sobre esta con ayuda, por supuesto, de la bibliografía en la que me he basado.

Palabras clave: traducción, feminista, género, feminismo, conciencia, patriarcal.

\section{Introducción}

La motivación principal de este trabajo reside en mi gran y creciente interés por los dos ámbitos de estudio en los que puede encuadrarse: los estudios de traducción e interpretación y el feminismo. A lo largo de estos años en el grado, me han surgido innumerables dudas sobre cómo poder llegar a hacer una buena traducción y preservar mi moral desde un enfoque feminista al mismo tiempo sin que pudiera parecer que cometía una y otra vez una simple y deliberada traición al texto origen. A pesar de que no eran textos problemáticos en cuanto a contenido, en la mayoría de ocasiones estas dudas estaban relacionadas con cuestiones de género que ahora, dado que tengo unos conocimientos más 
profundos y una ideología al respecto mejor fundada, etiquetaría como cuestiones relacionadas con el feminismo.

Tal y como Lori Saint-Martin $(1992,206)$ cita en su artículo "Susanne de Lotbinière-Harwood : Re-belle et infidèle : la traduction comme pratique de réécriture au féminin. The Body Bilingual : Translation as a Rewriting in the Feminine», existe una interesante analogía metafórica entre la traducción y la mujer: "Une traduction, c'est comme une femme; si elle est belle, elle risque d'être infidèle (...) infidèles à la loi du langage patriarcal.»

También otras traductoras y traductólogas feministas equiparan el estatus de la traducción, relegado a un segundo plano y considerado inferior, con la marginación actual de las mujeres, lo que desemboca en una doble discriminación (Bertacco 2003, 234; Calzada Pérez 2007, 104; Munday 2001, 131).

Por ello, podría hacerse una comparación, sin riesgo de que pareciera descabellada, entre el estatus de la figura de la traductora y de la mujer en sí misma. Ambas figuras quedan relegadas a un segundo plano en sus respectivos marcos de actuación, laboral y social en este caso. Por un lado, la traductora es considerada en muchas ocasiones como una máquina que simplemente se dedica a traducir y no siente ni padece; por otro lado, a ojos de una sociedad intrínsecamente patriarcal, se ve a la mujer como un instrumento a merced de dicha sociedad, alguien (o, en el peor de los casos, algo) sin voz ni voto.

A partir de estas relaciones analógicas que, consecuentemente y por fuerza, acaban derivando en la invisibilidad, opresión y menosprecio inequívoco de la figura femenina, se construye mi trabajo. Así, la relación entre la traductología y los estudios de género es más estrecha de lo que se podría pensar en un primer momento y me gustaría profundizar en los puntos de confluencia entre ambas disciplinas y en cómo la traductora puede convertirse en un motor de cambio social a través de su intervención en el texto origen.

Este trabajo ha de entenderse, pues, como una oportunidad para revisar y profundizar las cuestiones y los puntos más prácticos e importantes que a mi parecer se desprenden de la convergencia de la traducción y el feminismo.

\section{Objetivos}

El objetivo último y principal de este trabajo es una revisión de los conceptos que me parecen más importantes acerca de la traducción feminista, a través de lo que pretendo demostrar que el activismo feminista en el ámbito de la traducción y la interpretación es una práctica plausible que puede introducirse en la traducción de cualquier texto o la interpretación de cualquier discurso 
independientemente del contexto social, político y cultural, el idioma o la meta que persiga (siempre desde un enfoque interseccional que acomode a todos los grupos sociales que se representan). Además, deseo concienciar sobre la relación de las prácticas de traducción feminista con las estructuras de poder vigentes y su utilidad para acabar con estas y renovarlas.

De esta forma, analizaré detalladamente las relaciones entre feminismo y traducción, género y lenguaje y el importante papel del activismo, en este caso feminista, en la práctica traductora para romper con la percepción de la realidad social sexista derivada del empleo del lenguaje.

\section{Material y método}

Para realizar este trabajo necesité, en primer lugar, buscar las fuentes de documentación más adecuadas y relacionadas con los puntos que quería tratar, me documenté sobre autoras, teorías, estrategias y corrientes relacionadas con la traducción feminista, así como con el lenguaje, el género y el feminismo.

En segundo lugar, procedí a la lectura de dichas fuentes para poder conocer y contrastar diferentes perspectivas acerca de los temas relacionados con la traducción e interpretación, el lenguaje, el género y el feminismo. A medida que avanzaba con la lectura descubrí nuevos temas que me resultaban enriquecedores y descarté otros por ser, para mí, de orden secundario y por cuestiones de limitación de espacio. Así, me he basado en mi bibliografía y en mi concepción e ideas previas y experiencias acerca del tema de mi trabajo para poder delimitar los apartados definitivos de mi análisis teórico, teniendo en cuenta los temas que estaba interesada en tratar y prescindiendo de aquellos que no me parecían tan relevantes como para incluirlos en mi trabajo.

\section{Resultados}

\subsection{Género, lenguaje y conciencia}

Los textos se producen en un contexto ideológico y cultural concreto del que la traductora debe ser consciente en todo momento, pues la traducción es una práctica a la que afectan inevitablemente los factores políticos, sociales e ideológicos. Así, el contexto en el que se lleve a cabo la traducción es de gran relevancia, pues de él se desprenderán algunos aspectos (como los estereotipos sociales, las diferencias culturales o la idiosincrasia lingüística) dignos de tener en cuenta a la hora de traducir de forma congruente. Ser consciente de dicho contexto no conduce de manera 
irrevocable a cambiar el contenido, sino a tener conciencia por fin

del lenguaje con el que este se expresa y así poder analizarlo.

EI lenguaje estándar convencional es fundamentalmente patriarcal, ha sido creado por y para los hombres; von Flotow (1997) asevera que algunas escritoras de los setenta como Mary Daly o France Théoret opinaban lo siguiente: "Women live in exile in patriarchal language». Esta aserción puede parecer una exageración, pero el lenguaje, como bien apuntó Barthes (1953, citado por Monzó $2017,110)$ nunca es inocente, sino que es un arma poderosa que ha servido y continúa sirviendo como pilar fundamental para la construcción y el desarrollo de las estructuras de poder social y político que excluyen a las mujeres. Esta preeminencia del lenguaje patriarcal se consigue de formas más o menos subrepticias, entre las que destaca, por su permeabilidad y naturalización, el uso del masculino como genérico, que Goddard y Patterson $(2005,89)$ caracterizan del modo siguiente: «El uso de los términos masculinos en contextos supuestamente genéricos funciona para relegar a las mujeres del lenguaje, haciendo que sean "cognitivamente invisibles", es decir, que las excluye del pensamiento».

Así, a pesar de que el masculino genérico se intente vender como «neutral» y llegue avalado por instituciones patriarcales centenarias, nada más lejos de la realidad: se decanta activamente por un orden preestablecido de la sociedad patriarcal y entorpece la igualdad plena entre grupos sociales. Atendiendo al enfoque radical que he mencionado anteriormente, el hecho de emplear el masculino como genérico tiene como consecuencia directa la exclusión y no identificación de las mujeres dentro de la sociedad.

En realidad, como el resto de tácticas lingüísticas del patriarcado, el uso del masculino como genérico sería incluso anecdótico si no fuera porque el lenguaje construye y refleja una dimensión sociopolítica significativa, es decir, en una sociedad en la que no es extraño invisibilizar a las mujeres, sobre todo con miras a impedirles su acceso a espacios de poder varios, ocultarlas con el lenguaje puede entenderse como una argucia más para dificultar que eso ocurra siquiera como mera posibilidad.

Es importante, en este punto, mencionar la relación entre lenguaje y cognición, pues el lenguaje influye en el modo en el que organizamos nuestro sistema cognitivo, es decir, influye en la forma en que el cerebro organiza el pensamiento $y$ el conocimiento. $Y$ ejerce dicha influencia a través de un proceso de categorización, ya que la base del sistema cognitivo son las categorías.

En el caso del lenguaje patriarcal, se produce una categorización por género (masculino frente a femenino), a través de la que no solo se simplifican y homogeneizan grupos que son verdaderamente diversos, sino que también se incluye una categoría (femenina) dentro de otra (masculina) y se da mayor importancia a aquella que 
la sociedad patriarcal considera superior. Así, estas categorías realmente se crean en función de lo que la sociedad cree que es importante sobre las personas y, como indican Goddard y Patterson $(2005,75)$ :

hay pruebas de que si una categoría no es significativa para la sociedad no se emplea para describir o diferenciar a las personas, lo que también implica que cualquier categoría se podría volver importante en función de las convicciones que una sociedad tenga.

Por consiguiente, una traducción consciente en lo que a lenguaje y género respecta es esencial en el proceso traductor, pues puede ser capaz de revelar que el lenguaje no es fijo e inalterable y que las convicciones sociales igualmente pueden cambiar, $y$, desde esta conciencia lingüística de género, se puede iniciar y perfeccionar un importante proceso de deconstrucción del lenguaje y de la sociedad al mismo tiempo, y "la deconstrucción fuerza al máximo los límites del lenguaje. Se resiste a toda categorización y niega que en la traducción haya un significado único y estable que transmitir» (Vidal 1995, 91).

Las categorías, consecuentemente, son excluyentes. Aun así, la categoría masculina pretende abarcar en numerosas ocasiones tanto lo femenino como lo masculino; mientras que la femenina solo representa lo femenino. Las mujeres han de sentirse identificadas de forma indiscutible con la categoría masculina, mientras que los hombres nunca se sentirán de esta forma si se emplease una categoría femenina, que heriría gravemente su masculinidad. Este hecho desemboca innegablemente en una estructura de poder patriarcal: la idea de que no es legítimo ni razonable por parte de un hombre sentirse identificado con un grupo de mujeres, pero sí al contrario.

A pesar de que el uso de la categoría masculina pretende ser genérico y abarcar lo masculino y lo femenino, este uso del lenguaje conduce a implicaciones significativas desde un punto de vista cognitivo sobre todo porque se necesita un procesamiento 0 esfuerzo cognitivo adicional para percibir y entender que estamos ante un uso que pretende referirse a ambos géneros. Como afirman Goddard y Patterson $(2005,88)$ :

Este procesamiento secundario del significado genérico puede parecer bastante trivial si tenemos en cuenta el poco tiempo que tardamos en percibir un significado genérico frente a un significado exclusivamente masculino. Sin embargo, desde una perspectiva cognitiva y social, el impacto de esta primera lectura masculina tiene implicaciones muy importantes porque significa que pensamos en masculino a no ser que el contexto o algún marcador lingüístico nos indiquen lo contrario. 
Esta última frase ciertamente invita a la reflexión y a la toma de conciencia. Asimismo es una evidencia más del peso que tiene el lenguaje en la construcción cognitiva, social y moral del individuo.

Además, existen otras hipótesis que refuerzan esta noción de la relación entre lenguaje y cognición: la de Sapir-Whorf, dos estudiosos que llegaron a la conclusión de que aprehendemos la realidad a través del lenguaje. En otras palabras, ambos defienden que nuestras ideas sobre el entorno son el resultado del lenguaje que empleamos y que el lenguaje influye e incluso determina cómo percibimos la realidad y cómo pensamos (Gibbon 1999).

Así, la traducción feminista, que, de forma más o menos utópica, tiene en cuenta todos los aspectos anteriores en su puesta en práctica, ha de comprenderse como una forma más de emancipación de un sistema que toma al hombre como modelo al que equipararse y piensa que hombre siempre es sinónimo neutro de persona. De hecho, la traducción puede participar como engranaje dentro de la maquinaria del feminismo a fin de contribuir a la liberación de las cadenas del patriarcado. Ahora bien, para ello se ha de tener conciencia de género y esto, a la par que costoso, puede ser un desafío en toda regla debido a que toda la estructura social, política y cultural se yergue sobre unos cimientos firmes y bien asentados desde hace siglos. Por ello puede resultar complejo superar esta alienación lingüística, embarcarse en el proceso de deconstrucción y comprender el poder último que el lenguaje ejerce sobre toda la sociedad.

\subsection{Intervencionismo del traductor feminista}

Las traductoras, como el resto de individuos de la sociedad, poseen una ideología propia. Esto no significa que todas la reflejen de manera explícita en sus traducciones, pero cuando existe un posicionamiento firme como podría ocurrir con el feminismo, la conciencia feminista en muchas ocasiones se evidencia a través de la actividad traductora dando lugar a traductoras y traducciones indiscutiblemente politizadas de una manera $u$ otra.

El intervencionismo deliberado puede resultar controvertido, ya que se espera que las traductoras eviten incluir sus posicionamientos políticos en sus textos y sigan actuando como máquinas que trasladan información de un idioma a otro. Cuando las traductoras feministas actúan de acuerdo con su ideología se produce un desconcierto que pocas prácticas traductoras provocan y es que, como apunta Luise von Flotow $(1997,25)$ :

When feminist translators intervene in a text for political reasons, they draw attention to their action. In so doing, they demonstrate how easily misogynist aspects of patriarchal language can be dismantled once they have been detected. 
O bien como también opina Sherry Simon $(1996,12)$ sobre esta

misma cuestión: «Because it is an activity which has long been theorized in terms of a hierarchy of gendered positions, the rethinking of translation will necessarily upset traditional vocabularies of domination".

Así, la traducción de un texto se convierte en una práctica política relevante y en la materialización del encuentro entre la ideología feminista y el proceso traductor, cuyo objetivo es reescribir haciendo visible el femenino y lograr que el lenguaje represente también a las mujeres. Sin embargo, a menudo la traducción feminista se concibe como un acto de traición premeditada al texto origen que solo pretende destruirlo. Esta confusión recurrente es fruto una vez más de la alienación y del desconocimiento del poder del lenguaje. Sin embargo, el propósito del intervencionismo traductor feminista radica en visibilizar la presencia de la mujer a través del empleo de las palabras de las que dispone una lengua. Como afirman Goddard y Patterson (2005, 105):

La idea de «interferir» en el lenguaje resulta interesante, porque asume que el lenguaje es un producto natural más que una creación humana. Es fácil sentir que los orígenes del lenguaje están muy lejos en el tiempo y que el proceso de crear significado ya está terminado por completo. No obstante, este proceso es constante, delante de nuestras narices.

Es decir, la humanidad tuvo el poder de crear el lenguaje y por consiguiente también tiene el poder de cambiarlo si es necesario, pero para ello hay que ser consciente de la necesidad imperiosa de cambio y, sobre todo, de actuación.

Ahora bien, es cierto que hay cuestiones en cuanto al intervencionismo feminista para las que no existen respuestas universales concluyentes ni normas generales que se deban aplicar en cualquier caso, el intervencionismo depende enteramente de las decisiones de la traductora, pues como presenta Hurtado Albir (2001) en su libro Traducción y traductología: introducción a la traductología, traducir es un proceso de toma de decisiones y elección. Parte de la conciencia traductora reside, a mi parecer, en saber cuándo y cómo se debe «manipular» deliberadamente un texto, aunque toda traducción implique cierto grado de «manipulación» del texto origen en mayor o menor medida.

Por ejemplo, que un texto no sea abiertamente misógino ni ataque directamente a las mujeres no quiere decir que las mujeres se vean representadas en su contenido. En otras palabras, si el contenido del texto no está adscrito a ninguna ideología explícita, puede deducirse que esta falta de carga ideológica se traduce en una adscripción a la ideología dominante y que, por lo tanto, una traducción de esa misma naturaleza también estará subordinada a la 
ideología dominante: la patriarcal. Las traductoras que no son conscientes de esta ideología dominante se convierten entonces en «ingenuos vehículos de transmisión y legitimación del discurso dominante» (Castro Vázquez 2009, 63).

Además, cuando se intervenga un texto se ha de tener en cuenta que la perspectiva de partida no es siempre la de un feminismo puramente occidental (como podría ocurrir al traducir obras de autoras indias o africanas) y que dentro del propio movimiento feminista será necesario "observar las diferencias entre las diversas experiencias femeninas atendiendo a las distinciones de las mujeres en cuestión y enfatizando la necesidad de contextualizar" (Salvador 2006), pues siempre habrá diferentes posturas, enfoques y estadios que valorar antes de abordar la traducción.

Así, las decisiones personales de la traductora, aquellas que están motivadas por su ideología y su moral feminista, tienen un gran peso en el proceso traductor en general y en las soluciones concretas en particular a pesar de no ser ninguna de ellas inocente ni inocua (Brufau Alvira 2011, 193). Si algo queda claro es que el intervencionismo feminista como acción política y la incorporación de la ética traductora desde una perspectiva de género son prácticas viables en la traducción de cualquier texto y que, además, citando a Olga Castro $(2009,63)$, «la objetividad y neutralidad en traducción son falacias interesadas».

\subsection{La traductora infiel}

Uno de los escollos con los que más a menudo se topa la traductora feminista es, sin lugar a dudas, el cuestionamiento externo. La traducción feminista se enfrenta a las prácticas traductoras clásicas $y$, por consiguiente, es objeto de estigmatización. En muchas ocasiones, aplicar una perspectiva de género e intentar evitar el sexismo y androcentrismo lingüístico «no solo comporta dificultades lingüísticas, sino otras de índole social que se mezclan en el maremágnum político común» (Brufau Alvira 2011, 193).

No es disparatado que la traductora, abogando por ser fiel a su conciencia, opte por una traducción feminista. Si se decanta por la reescritura en femenino o incluso por estrategias menos agresivas de inclusión lingüística, esto la posicionará políticamente, de manera voluntaria o involuntaria. A ojos de una sociedad y unas instituciones patriarcales, este comportamiento supone una infidelidad no solo al texto sino también a los constructos sociales.

Como consecuencia, la actividad traductora feminista se enfrenta continuamente a una serie de dilemas (muchas veces dicotómicos) a varios niveles: fidelidad-infidelidad, naturalidadartificialidad, equivalencia-reformulación, legitimidad-engaño, que parecen no tener fin y ante los que las decisiones personales basadas 
en la ideología de la traductora se presentan como el único desenlace lógico. Si se adopta un enfoque traductor feminista radical, la solución resulta más que obvia: hay que subvertir y socavar el texto de manera sistemática si este ataca a nuestros ideales, no se necesitan motivos categóricos para actuar, porque tal y como describe von Flotow a de Lotbinière-Hardwood: "She offers no excuses or justifications for feminist intervention in texts, but an abudance of compelling reasons» (de Lotbinière-Hardwood, citada en von Flotow 1997, 27).

Las críticas de los detractores sobre esta reescritura o reformulación en femenino ponen en tela de juicio su validez y dificultan la expansión de su práctica. Incluso cuando se emplean estrategias menos agresivas que la reformulación total como técnicas de inclusión o generalización, se encuentran opositores que denuncian la transgresión a la economía lingüística, tan interiorizada y cómoda para muchas instituciones patriarcales; o la artificialidad y falta de idiomatismo del texto meta, que en realidad es producto de la falta de costumbre por incluir prácticas traductoras distintas a las tradicionales.

A raíz de estas acusaciones de infidelidad o incluso traición al texto original, surge una polémica dicotomía fidelidad-infidelidad: si la traductora es totalmente fiel al texto origen, corre el riesgo de ser infiel a su ideología y viceversa. Cuando la traductora se decanta por serle infiel al texto y mantenerse fiel a su moral feminista ocurre lo que Martín Ruano (citada en Calefato y Godayol 2008, 51) define como "fidelidad subversiva aunque "fidelidad" a fin de cuentas".

Por supuesto, una de las tareas de la traductora es que el texto traducido sea natural, idiomático y una reproducción lo más exacta posible del original, así que ¿no supone en este punto la traducción feminista un obstáculo evidente? El sobreempleo de genéricos y epicenos puede resultar poco familiar, el uso de barras y dobletes puede llegar a hastiar a quien reciba el texto meta y la reescritura o reformulación en femenino, con total probabilidad, causará un desconcierto enorme en el público. Estos hechos llevarán a la traductora a enfrentarse de forma continuada a situaciones delicadas en su trabajo y a rivalizar con un mundo en el que se premia y legitima una aparente normalidad asentada en unos rancios valores patriarcales.

Ahora bien, la traductora feminista, subversiva e infiel, lejos de tirar la toalla siempre puede buscar fuerza y consuelo en las palabras de Susanne de Lotbinière-Hardwood (citada en von Flotow 1997, 79; 1991, 29): "Making the feminine visible in language means making women seen and heard in the real world, which is what feminism is all about». 


\subsection{Aplicación de la traducción feminista en la interpretación}

A pesar de que pueda resultar obvio, la forma de trasladar un mensaje de forma no sexista y la reformulación en femenino pueden ser más problemáticas en el campo de la interpretación. Esto ocurre principalmente en interpretación simultánea, porque en ella no se dispone de un periodo de tiempo para la reflexión ni para el análisis minucioso de las palabras.

Incluso a partir de mi corta experiencia en el área de la interpretación, he detectado que existen dos problemas principales y recurrentes a los que hacer frente y los he plasmado en una tabla que se encuentra más adelante (tabla 1). En primer lugar, surge una disyuntiva cuando en una lengua con género gramatical explícito (como el español) se presenta un (falso) masculino genérico ante el que la traductora debe decidir si hacer un desdoble masculinofemenino o mantener el masculino (1). En segundo lugar, esto mismo puede suceder por supuesto en el caso contrario, si se trabaja desde una lengua en la que no suele haber marca de género (como el inglés) (2).

Para ilustrar los casos anteriores me he basado en algunos de los discursos (FEND Lectures y On Being Wrong) que hemos utilizado en la asignatura de Interpretación Simultánea (2) y también en un ejemplo (1) que proporciona Olga Castro (2009), aunque he de apuntar que su ejemplo no tiene la intención de ilustrar casos que se dan en interpretación concretamente, sino en una traducción en general.

Tabla 1.

\begin{tabular}{|c|c|c|c|c|}
\hline & ES & EN & FR & DE \\
\hline 1 & Mis tíos & $\begin{array}{l}\text { My uncle and } \\
\text { aunt } \\
\text { My uncles } \\
\text { My aunt and } \\
\text { uncles } \\
\text { My aunts and } \\
\text { uncle }\end{array}$ & $\begin{array}{l}\text { Mon oncle et ma } \\
\text { tante } \\
\text { Mes oncles } \\
\text { Ma tante et mes } \\
\text { oncles } \\
\text { Mes tantes et } \\
\text { oncles }\end{array}$ & $\begin{array}{l}\text { Mein Onkel und } \\
\text { meine Tante } \\
\text { Meine Onkel } \\
\text { Meine Tante und } \\
\text { meine Onkel } \\
\text { Meine Tanten } \\
\text { und meine Onkel }\end{array}$ \\
\hline 2 & $\begin{array}{l}\text { Enfermera } \\
\text { Enfermero }\end{array}$ & Nurse & $\begin{array}{l}\text { Infirmière } \\
\text { Infirmier }\end{array}$ & $\begin{array}{l}\text { Krankenschweste } \\
\text { r Krankenpfleger }\end{array}$ \\
\hline & $\begin{array}{l}\text { Amiga } \\
\text { Amigo }\end{array}$ & Friend & $\begin{array}{l}\text { Amie } \\
\text { Ami }\end{array}$ & $\begin{array}{l}\text { Freundin } \\
\text { Freund }\end{array}$ \\
\hline
\end{tabular}

En estos casos, existe un riesgo evidente de incurrir en una traición al TO tanto si se opta por la traducción feminista como si no, 
pues la situación es de desconocimiento total del género del sustantivo que la oradora ha mencionado.

Otra de las trabas con respecto a la práctica de traducción feminista y no sexista en la interpretación es el tiempo. En la interpretación simultánea el tiempo puede ser nuestro mayor aliado o nuestro peor enemigo: mejor dicho, el desfase temporal con respecto al TO. El hecho de querer deshacernos del lenguaje sexista en nuestra reformulación del discurso puede conducirnos a aumentar dicho desfase y provocar que, por ejemplo, ya no podamos reengancharnos al ritmo del discurso original, lo que puede ser muy peligroso. No es lo mismo decir: «doy la bienvenida a todas las enfermeras de Europa a esta conferencia» que "doy la bienvenida a todas las enfermeras y enfermeros de Europa a esta conferencia» o que «doy la bienvenida al personal de enfermería de Europa a esta conferencia».

Cuando se dan casos como los anteriores, la traductora se ve de nuevo rodeada de dilemas en lo que a la manera de reformular respecta. Sin embargo, el proceso de interpretación no deja tiempo para que se reflexione acerca de qué decisión tomar con tranquilidad, por lo que la solución para una traductora con un posicionamiento ético feminista firme (radical) sería, por supuesto, la reformulación en femenino y el empleo de lenguaje inclusivo en todo momento, a riesgo de que esta práctica tan poco convencional choque a la audiencia.

\subsection{Traducir en femenino: estrategias}

Como vengo manifestando a lo largo de todo mi escrito, la traductora feminista tiene el poder de «manipular» el texto si lo considera oportuno e incluir en él su ideología para liberarlo de su carga patriarcal. Para conseguir este objetivo, puede recurrir al empleo de diferentes estrategias textuales y discursivas de traducción feminista. Me basaré principalmente en los ejemplos y las estrategias propuestas por Castro Vázquez (2010) en su artículo "Género y traducción: elementos discursivos para una reescritura feminista", puesto que me han parecido tan prometedoras como aplicables.

En primer lugar, una de las estrategias más recurrentes para evitar el lenguaje no sexista es la generalización o neutralización. Consiste en sustituir el término sexista por uno neutro o un epiceno que designe de igual manera a individuos de ambos géneros independientemente del género gramatical del sustantivo (estudiante, bebé). Así se evita indicar el género de forma explícita. Sin embargo, se ha de tener en cuenta la tendencia a entender los sustantivos neutros como masculinos como consecuencia del «MaleAs-Norm Principle» o «MAN Principle», por el cual se suele optar por el masculino si se desconoce el género del sujeto. 
Otra técnica es la feminización o especificación. Esta práctica supone visibilizar las marcas de género gramatical para también visibilizar a las mujeres en el lenguaje; es decir, aparece el género correspondiente al referente que se ha mencionado (masculino si es hombre, femenino si es mujer, ambos géneros si son mujer y hombre o si hay desconocimiento del género del referente). Algunos de los recursos mencionados por Castro Vázquez son paréntesis, barras, guiones, mayúsculas, arrobas y dobletes. Me gustaría hacer una añadidura, una innovación más o menos reciente: el empleo de la $\boldsymbol{x}$ para sustituir a las letras que funcionan como marca de género y que se emplea principalmente en las redes sociales (chicxs; queridxs alumnxs; amigxs). Realmente la considero una renovación y mejora de la arroba, pues es más discreta dentro del conjunto textual (llama menos la atención porque la arroba es más grande y ni siquiera es una letra) y, además, en la arroba la $\boldsymbol{a}$ parece estar contenida o, cuando menos rodeada, por la $\boldsymbol{o}$, lo cual podría representar gráficamente la subyugación del mundo femenino por el masculino.

Las estrategias anteriores son las más conocidas y habituales para hacer uso de un lenguaje más inclusivo y no sexista, tanto en la práctica de traducción feminista como en otras prácticas traductoras. A continuación voy a presentar varias técnicas que también pueden resultar muy útiles en la práctica traductora feminista y que más bien se encuadran en un enfoque radical:

- La suplementación o compensación. Consiste en compensar las diferencias entre lenguas y culturas intentando conservar el significado y las connotaciones del original. Algunas reconocidas traductoras feministas como LotbinièreHardwood, que resaltó las marcas de género del francés al traducir al inglés, o Goddard, que traduce el libro Amantes como LoveHers para advertir que se trata de lesbian lovers, son claros ejemplos de la puesta en práctica de esta estrategia.

- La metatextualidad. Esta práctica se basa en incluir prefacios, notas de la traductora, prólogos y otros paratextos que expliquen y justifiquen el intervencionismo que se ha llevado a cabo en el texto. A través de esta estrategia, la traductora también consigue dar visibilidad a su actividad. Uno de los ejemplos más representativos de esta técnica es el prólogo de Susanne de LotbinièreHardwood en la que explica el uso de her en other, pues tradujo Lettres d'une autre (1987), de Lise Gauvin, como Letters from An Other. En dicho prólogo (de LotbinièreHarwood, en Gauvin, 1989, citado en von Flotow 1997, 29) dejaba clara su ideología feminista: 
Dear reader,

Just a few words to let you know that this translation is a rewriting in the femenine of what I originally read in French. I don't mean content. Lise Gauvin is a feminist, and so am I. But I am not her. She wrote in the generic masculine. My translation practice is a political activity aimed at making language speak for women. So my signature on a translation means this translation has used every possible feminist translation strategy to make the femenine visible in language.

- El secuestro. Es una estrategia por la que la traductora se apropia de un texto con lenguaje patriarcal y lo reescribe utilizando neologismos, introduciendo cambios, sustituyendo el masculino genérico por el femenino genérico, eliminando elementos sexistas, resaltando algunos elementos... Un ejemplo de esta modalidad es el mismo que el de la anterior, pues la traducción de Lotbinière-Hardwood de Lettres d'une autre es una reescritura en femenino usando el secuestro en toda regla; o también la traducción de textos de Cabrera Infante que llevó a cabo Suzanne Jill Levine (citado en Castro 2009)

- La coautoría o pacto especular, término acuñado por MarieFrance Dépêche (2002, citada en Castro Vázquez 2010, 296). Esta cuarta estrategia traductora feminista consiste en la colaboración entre quien escribe y quien traduce, de manera que se consiga la coautoría de la obra traducida. Esta técnica se puso en práctica en la traducción de la obra de Nicole Brossard (1998) Elle serait la première phrase de mon prochain roman / She would be the first sentence of my next novel, que Lotbinière-Harwood tradujo al inglés.

Junto con la feminización y la generalización, estas cuatro estrategias de suplementación, metatextualidad, secuestro y coautoría constituyen la puesta en práctica de la ideología y los principios éticos y morales de la traductora feminista, así como la materialización de un empeño incesante por hacer a la mujer visible a través del lenguaje $y$, por ende, visible en la sociedad. Desde este punto de vista en el que la traductora desempeña un papel activo en el proceso de traducción y opta por la práctica de algunas de las técnicas y estrategias anteriores, Goddard (citada en Bertacco, 2003: 237) afirma lo siguiente: "translation detaches itself more and more from the ideal of quest for equivalence and, instead, presents transformation as performance as its model».

También es importante mencionar que en España, instituciones, asociaciones y otros organismos como la Secretaría General de Políticas de Igualdad, el Instituto de la Mujer y la Federación de 
Mujeres Progresistas han elaborado manuales de escritura no sexista

(Brufau Alvira, 2011: 191). Existe un recurso informático, una base de datos más concretamente, elaborada por el grupo NOMBRA (Comisión Asesora sobre Lenguaje del Instituto de la Mujer) que facilita opciones alternativas de escritura para evitar el lenguaje sexista y se puede descargar de manera gratuita. Esta herramienta consta de tres pestañas: palabras ante las que conviene extremar el cuidado (imagen 1), una lista general de expresiones de uso problemático (imagen 2) y expresiones frecuentes (imagen 3). En la primera de ellas, además de opciones para sustituir el término en masculino por otros inclusivos, se muestran el contexto original en el que se encuentran la palabra o palabras y el contexto alternativo con la nueva opción. En la tercera, junto con los contextos, se muestra un apartado de ejemplos en corpus.

Iniciativas como la de NOMBRA impulsan el empleo de lenguaje inclusivo y constituyen herramientas útiles e innovadoras en pro de la eliminación gradual del lenguaje sexista y androcentrista.

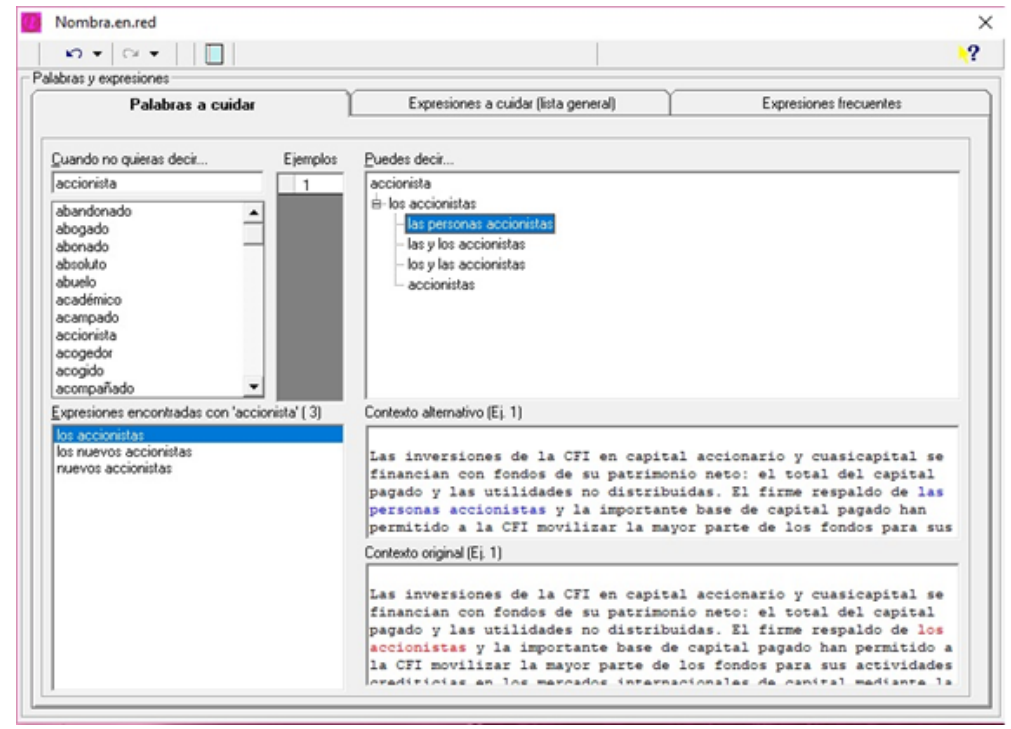

Imagen 1 


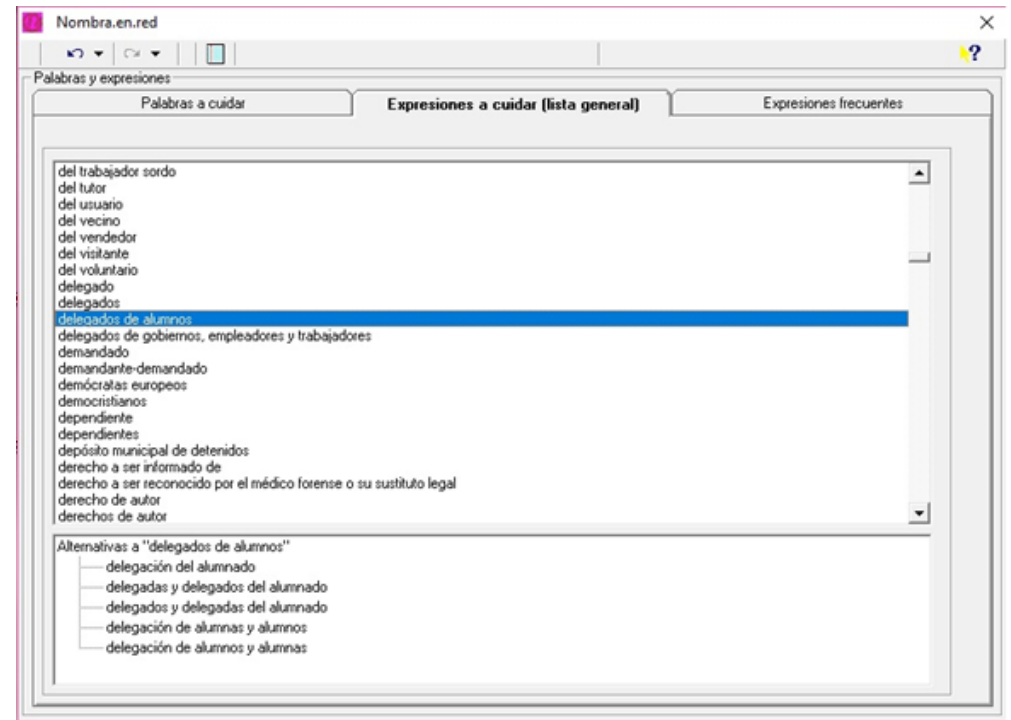

Imagen 2

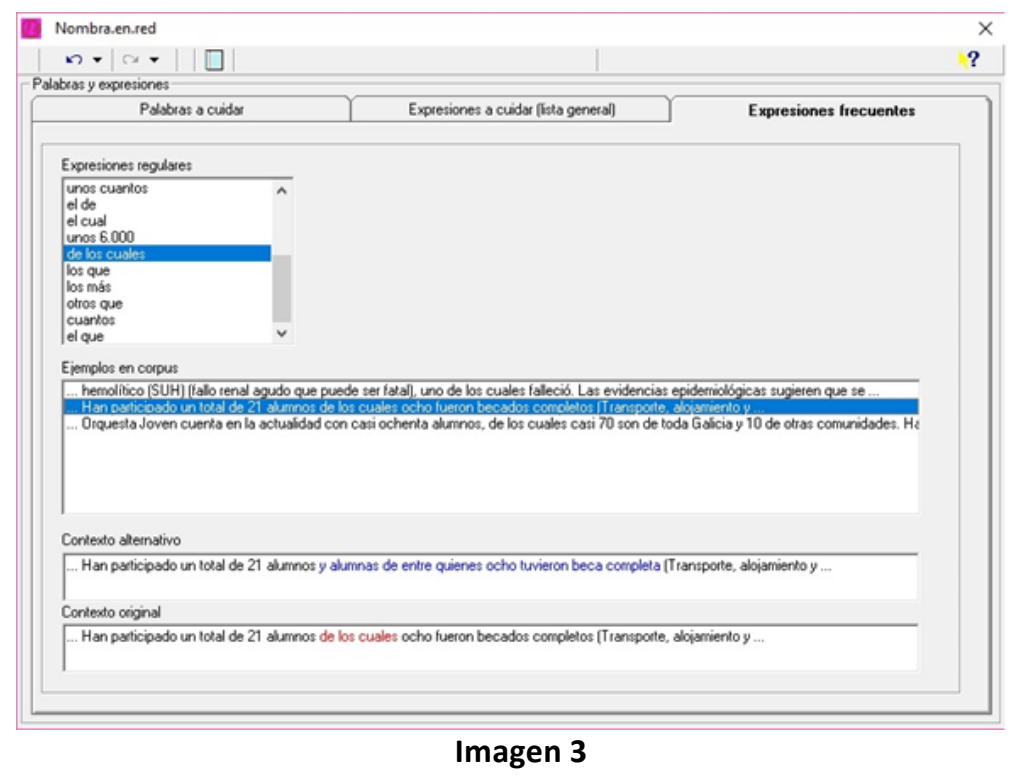

\section{Discusión y conclusiones}

Concluyo este trabajo con la certeza de que ha sido un incipiente proceso de introspección que me ha servido como punto de partida para comenzar a adentrarme en la traducción feminista, un campo de estudio que me fascina y sobre el que llevaba tiempo queriendo saber más. A medida que me he sumergido en las páginas de escritoras, en general todas ellas feministas, no solo he aprendido acerca de feminismo y traducción, sino que me he dado cuenta de que ambos campos de estudio están estrechamente relacionados con otras cuestiones que no habría podido imaginar, como por ejemplo la cognición.

Soy consciente de que los objetivos no eran extremadamente ambiciosos y de que las cuestiones que he revisado son las que a mí 
más me llamaban la atención. Así que, llegada a este punto, puedo aseverar que la hipótesis queda confirmada: existe una relación patente entre feminismo y traducción, en otras palabras, entre los estudios de género y los ET, que se materializa en la traducción feminista. Además, la puesta en práctica de la traducción feminista, es decir, introducir el intervencionismo y la conciencia feminista en las prácticas de traducción es posible en mayor o menor medida dentro del marco sociopolítico actual a pesar de su falta de normalización (que espero vaya disminuyendo de forma progresiva). Queda también comprobado que se trata de una actividad política y social por los objetivos que persigue, entre los que están principalmente dar visibilidad y voz a la mujer dentro de una sociedad patriarcal que quiere acallarla y tratar de poner fin a la perpetuación de las estructuras de poder impuestas por esta sociedad.

Las conclusiones teóricas a partir del trabajo conducen a su vez a reflexiones de índole más bien social, política y moral, pues me han permitido ratificar la importancia de tener una postura ideológica firme. Leer experiencias reales de traductoras y ejemplos concretos de traducciones feministas ha sido clave para una mejor comprensión de lo que realmente significa subvertir un texto y hacerlo tuyo como traductora. Esto ha ocurrido gracias a la lectura de las obras de traductoras feministas, mujeres deconstruidas, subversivas y con una ideología fundada, como LotbinièreHardwood, Goddard, Simon, Castro Vázquez, Brufau Alvira, etc., que se han encargado de articular y desplegar una amplia maquinaria de teoría y praxis en lo que a traducción feminista respecta, de modo que han permitido que esta continúe creciendo cada día a través del interés, el estudio y la lucha de las nuevas generaciones de traductoras feministas.

La elaboración de este trabajo ha sido, en definitiva, un proceso de (de)construcción constante que, además de ampliar mis conocimientos en estas áreas de estudio, me ha permitido crecer como estudiante, como traductora y como persona.

\section{Bibliografia}

de Beauvoir, Simone. 2017. Le deuxième sexe. París: Gallimard.

Bertacco, Simona. 2003. "The Canadian Feminists' Translation Project: Between Feminism and Postcolonialism». Linguistica Antverpiensia, New Series - Themes in Translation Studies 2: 233-245.

Brufau Alvira, Nuria. 2011. "Traducción y género: el estado de la cuestión en España». MonTI. Monografías de Traducción e Interpretación 3: 181-207. 
Calefato, Patrizia, y Pilar Godayol. 2008. Traducción, género, poscolonialismo. Buenos Aires: La Crujía.

Calzada Pérez, María. 2007. El espejo traductológico: teorías y didácticas para la formación del traductor. Barcelona: Octaedro.

Castro Vázquez, Olga. 2009. "(Re)examinando horizontes en los estudios feministas de traducción: ¿hacia una tercera ola?» MonTI. Monografías de Traducción e Interpretación 1: 59-86.

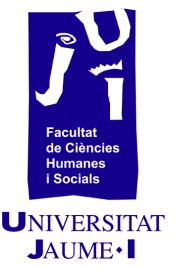

—. 2010. "Género y traducción: elementos discursivos para una reescritura feminista». Lectora: Revista de Dones i Textualitat 14: 285-302.

von Flotow, Luise. 1991. "Feminist Translation: Contexts, Practices and Theories». TTR: Traduction, Terminologie, Rédaction 4 (2): 69-84.

-. 1997. Translation and gender: translating in the "era of feminism». Manchester, Ottawa: St. Jerome Publishing; University of Ottawa Press.

Gibbon, Margaret. 1999. Feminist Perspectives on Language. Feminist Perspectives Series. Londres: Addison Wesley Longman.

Goddard, Angela, y Meân Patterson. 2005. Lenguaje y género. Cuenca: Universidad de Castilla-La Mancha.

Hurtado Albir, Amparo. 2001. Traducción y traductología: introducción a la traductología. Madrid: Cátedra.

Monzó Nebot, Esther. 2017. «The out-grouping society: phrasemes othering underprivileged groups in the International Bill of Human Rights (English-French-Spanish)». Phraseology in legal and institutional settings: a corpus-based interdisciplinary perspective, editado por Stanislaw Goźdź-Roszkowski y Gianluca Pontrandolfo, 131-159. Londres: Routledge

Munday, Jeremy. 2001. Introducing translation studies: theories and applications. Londres; Nueva York: Routledge.

Saint-Martin, Lori. 1992. "Susanne de Lotbinière-Harwood: Re-belle et infidèle: la traduction comme pratique de réécriture au féminin. The Body Bilingual: Translation as a Rewriting in the Feminine». Recherches féministes 5 (1): 204-207.

https://doi.org/10.7202/057690ar.

Salvador, Dora Sales. 2006. "Traducción, género y poscolonialismo: compromiso traductológico como mediación y affidamento femenino». Quaderns: Revista de traducció 13: 21-30.

Sau, Victoria. 1990. Diccionario ideológico feminista. 2a. ed. ampliada y rev. Barcelona: Icaria. 
Schulz, Kathryn. 2011. «On being wrong». Vídeo de TED Talks. Acceso https://www.ted.com/talks/kathryn_schulz_on_being_wrong.

Simon, Sherry. 1996. Gender in translation: cultural identity and the politics of transmission. Londres: Routledge.

Vidal, M. Carmen África. 1995. Traducción, manipulación, desconstrucción. Salamanca: Ediciones Colegio de España. 
